\title{
Verification and Uncertainty Reduction of Amchitka Underground Nuclear Testing Models
}

\author{
prepared by
}

Ahmed Hassan and Jenny Chapman

\author{
submitted to \\ Nevada Site Office \\ National Nuclear Security Administration \\ U.S. Department of Energy \\ Las Vegas, Nevada
}

February 2006

Publication No. 45216 
Reference herein to any specific commercial product, process, or service by trade name, trademark, manufacturer, or otherwise, does not necessarily constitute or imply its endorsement, recommendation, or favoring by the United States Government or any agency thereof or its contractors or subcontractors. The views and opinions of authors expressed herein do not necessarily state or reflect those of the United States Government or any agency thereof.

Available for sale to the public, in paper, from:

U.S. Department of Commerce

National Technical Information Service

5285 Port Royal Rd.

Springfield, VA 22161

phone: 800.553 .6847

fax: 703.605.6900

email: order@ntis.gov

online ordering: http://www.osti.gov/ordering.htm

Available electronically at http://www.osti.gov/bridge

Available for a processing fee to the U.S. Department of Energy and its contractors, in paper, from:

U.S. Department of Energy

Office of Scientific and Technical Information

P.O. Box 62

Oak Ridge, TN 37831-0062

phone: 865.576 .8401

fax: 865.576 .5728

email: reports@adonis.osti.gov 


\title{
Verification and Uncertainty Reduction of Amchitka Underground Nuclear Testing Models
}

\author{
prepared by \\ Ahmed Hassan and Jenny Chapman \\ Division of Hydrologic Sciences \\ Desert Research Institute \\ Nevada System of Higher Education \\ Publication No. 45216 \\ submitted to \\ Nevada Site Office \\ National Nuclear Security Administration \\ U.S. Department of Energy \\ Las Vegas, Nevada
}

February 2006

The work upon which this report is based was supported by the U.S. Department of Energy under Contract \#DE-AC52-00NV13609. Approved for public release; further dissemination unlimited. 
THIS PAGE INTENTIONALLY LEFT BLANK 


\section{EXECUTIVE SUMMARY}

The groundwater flow and radionuclide transport at the Milrow, Long Shot and Cannikin underground nuclear test sites on Amchitka Island were modeled in 2002 using two-dimensional numerical simulations. A multi-parameter uncertainty analysis was adapted and used to address the effects of uncertainties associated with the definition of the modeled processes and the values of the parameters governing these processes. This stochastic modeling framework propagated uncertainty in input parameters through flow and transport simulations to yield an output with a wide range of uncertainty.

The Consortium for Risk Evaluation with Stakeholder Participation (CRESP) undertook a major field expedition in summer 2004 that yielded data pertaining to the location of the freshwater lens, derived from geophysical surveys on the island. Of particular importance to this study is the magnetotelluric (MT) data that were collected on profiles that passed through each of the three test sites. Interpretation of these data provided lateral profiles of the upper and lower boundaries of the freshwater-seawater transition zone at the three sites. In addition, analysis of geophysical data provided new information regarding effective fracture porosity. Updated bathymetric information was also collected for Cannikin and Long Shot. The purpose of the current investigation is to use these newly collected data sets for verifying the 2002 model and reducing the uncertainty associated with model input parameters and subsequent model predictions.

A statistical Bayesian framework is used to solve the inverse problem and develop the posterior distributions of the groundwater flow model parameters at the three sites. The Bayesian statistical paradigm recognizes that there are two sources of information about model parameters: 1) prior information that is based on expert knowledge for similar cases, and 2) data collected in the area of study that pertain to the processes modeled. This framework produces a probability density function for the model parameter that is called the posterior distribution. This distribution is a function of the prior distribution and a likelihood function that simply is a measure of the mismatch between model predictions and observed data.

This Bayesian approach is implemented here using a Markov Chain Monte Carlo (MCMC) sampling method, which generates enough samples from a random walk, and adapts to the true posterior distribution of parameters. These MCMC methods do not give a closed form for the posterior distribution, but rather give a large number of samples of the model parameters, which converge to the right posterior distribution. In other words, this approach develops parameter distributions conditioned on prior knowledge plus the available data. The data used for conditioning include the original chemistry and head data from wells at the three sites and the newly collected CRESP data pertaining to the location of the transition zone.

Numerous test runs and sensitivity analyses are conducted to understand the impact of each data set separately and the combined impact of the multiple data sets available for conditioning (chemistry, head, and MT data). Alternatives of the conditioning sequence and alternatives of combining data sets together are evaluated to identify the conditioning approach using the available data sets. A sequential conditioning approach is implemented where the chemistry data and MT-converted concentrations are combined as a single data set 
and are used to first condition the parameter distribution using as prior knowledge the uncertain distributions used in the 2002 models. The head data are subsequently used as a second conditioning data set where the posterior distribution developed by the first conditioning is used as a prior for this second conditioning.

The results of this conditioning include a posterior distribution for the recharge and a posterior distribution for the hydraulic conductivity. A corresponding posterior distribution for the recharge-conductivity ratio results when the recharge and conductivity values generated simultaneously from the MCMC algorithm are paired. Results of this analysis indicate that conditioning on the MT-converted concentrations and the head and chemistry data yields dramatic reduction of uncertainty, especially for the recharge-conductivity ratio. This ratio controls the location of the transition zone, and given that the MT data provide a good lateral profile for the transition zone boundaries, the transition zone location is constrained by the data and the resulting recharge-conductivity ratio has a smaller range of variability compared to the values used in the 2002 model.

The conductivity, recharge, and conductivity-recharge ratio posterior distributions, generated by conditioning on the data, fit within the distributions employed in the 2002 model. None of the conditioned distributions shifted away from the original distributions used in the 2002 model. This provides verification of the 2002 model parameter distributions. Using these conditioned distributions to solve the density-driven flow problem in a stochastic framework results in a range of output flow fields that are statistically analyzed at the locations where well data exist. The ensemble mean of these Monte Carlo solutions and the uncertainty bounds expressed by the mean \pm one standard deviation lie within the uncertainty bounds of the 2002 model. This is the case for many of the well data available with the exception of the salinity profile at UAe-1 at Cannikin, where the new bounds for low concentrations are outside the original model bounds. Other profiles at Cannikin, however, verify the original model, where the new results fit within the 2002 model uncertainty bounds.

Transport simulations are then conducted using the same conceptual transport model and processes as the 2002 model. The new flow realizations are combined with the porosity profiles provided in a 2005 study based on the analysis of the MT data. These porosity profiles show porosity decay with depth but the overall range of the porosity is much higher than the range used in 2002. The porosity distributions used in the 2002 model were skewed toward low values to be conservative. As a result, groundwater velocities in the new model are orders of magnitude slower than the 2002 model, resulting in essentially no radionuclide arrival to the seafloor in a simulation time of 2,200 years. These results are obtained without including any of the transport-retarding processes (i.e., sorption, matrix diffusion, or radioactive decay).

Using the new CRESP data and combining them with the head and chemistry data resulted in a dramatic reduction of model parameter uncertainty. This reduction propagated through the flow model to the location of the transition zone, which has a much tighter range compared to the original uncertain range. Groundwater fluxes are also associated with a tighter uncertainty range where both ends of the flux distributions are trimmed by conditioning. Uncertainty reduction in radionuclide mass flux could not be assessed, as no breakthrough occurred in the simulation time frame. 


\section{CONTENTS}

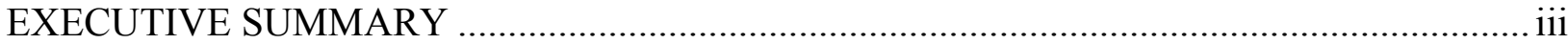

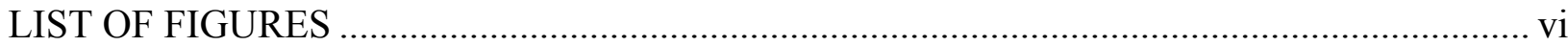

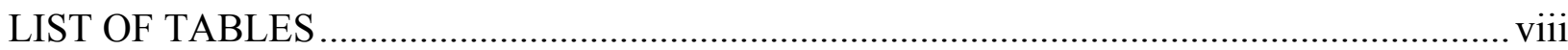

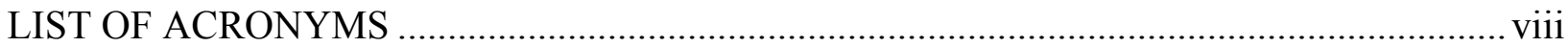

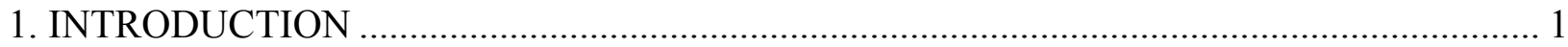

2. OVERVIEW OF THE ORIGINAL GROUNDWATER MODEL .................................... 3

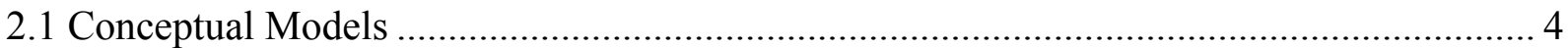

2.2 Processes Modeled, Parameters, and Calibration ................................................. 6

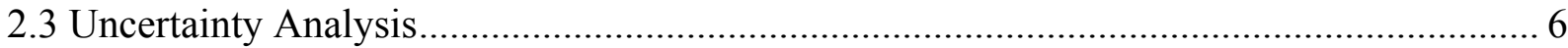

3. BAYESIAN MARKOV CHAIN MONTE CARLO METHOD: THEORETICAL

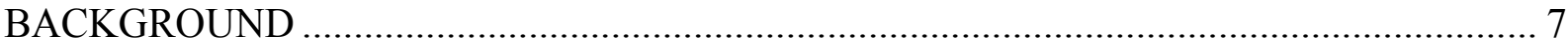

3.1 The Inverse Problem (Calibration Problem) .............................................................. 7

3.2 Bayesian Statistical Inference ............................................................................ 8

3.3 Markov Chain Monte Carlo ...................................................................................... 10

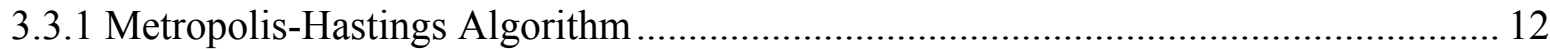

3.3.2 Adaptive Metropolis Algorithm.......................................................................... 13

4. RECALIBRATION, VERIFICATION AND UNCERTAINTY REDUCTION USING

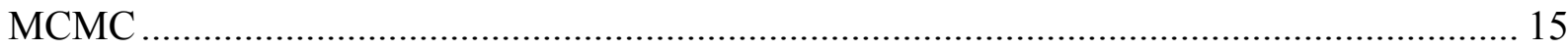

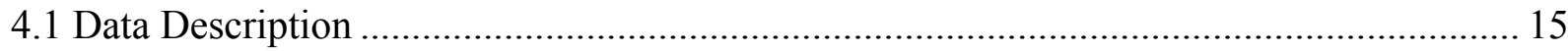

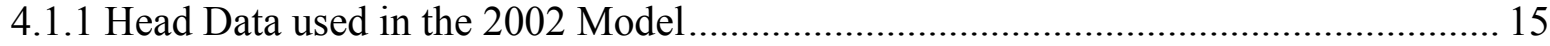

4.1.2 Salinity Data used in the 2002 Model................................................................... 17

4.1.3 Magnetotelluric Data Collected by CRESP in 2004 ............................................ 18

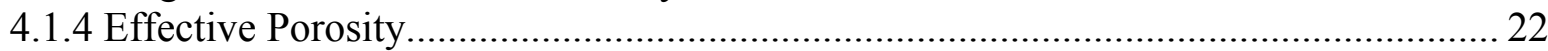

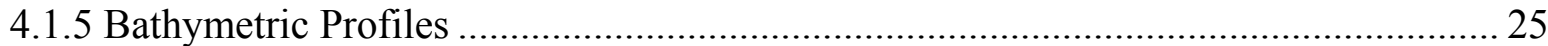

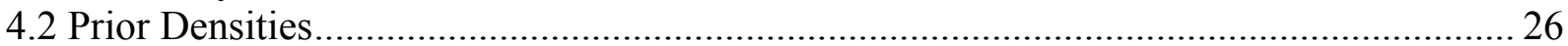

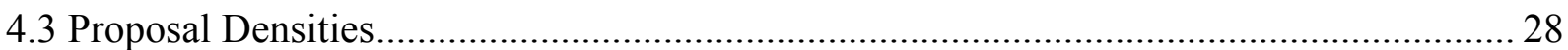

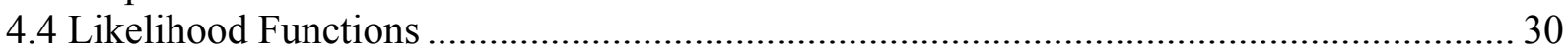

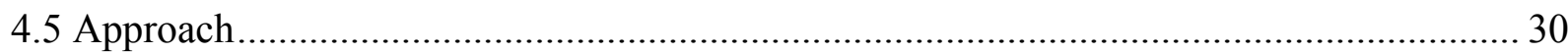

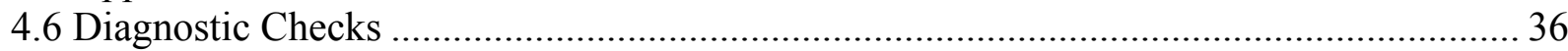

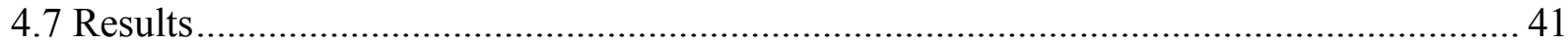

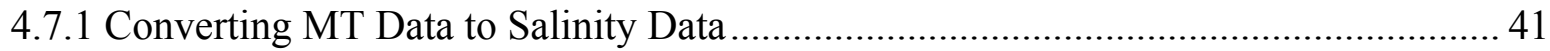

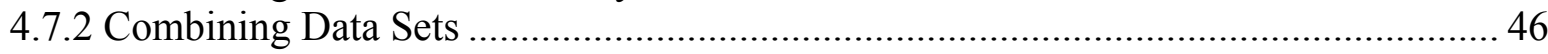

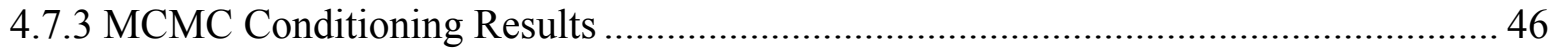

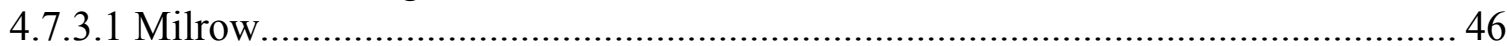

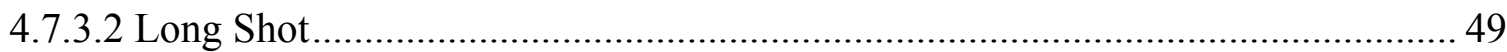

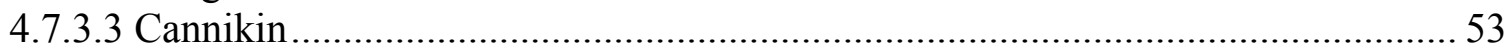

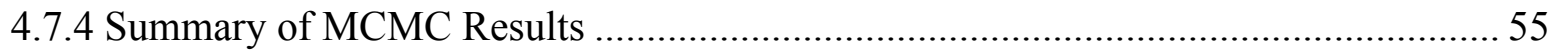

5. REVISED FLOW MODELING ............................................................................... 57

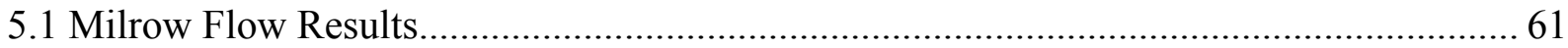

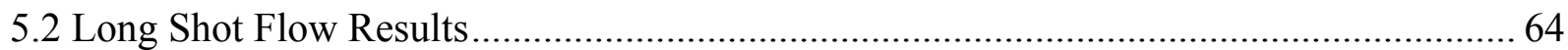

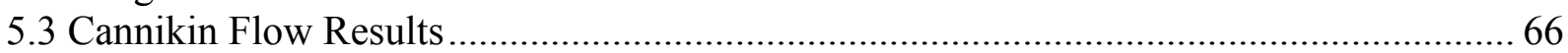

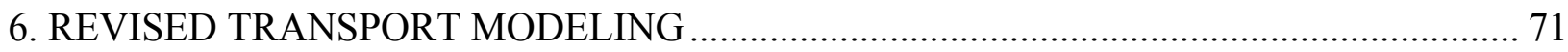

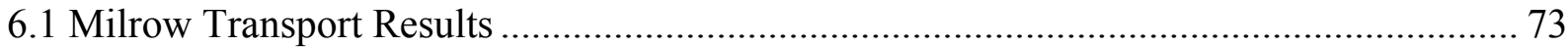

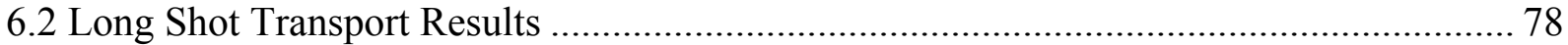

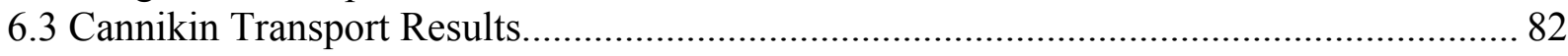


7. SUMMARY OF MODEL VERIFICATION AND UNCERTAINTY REDUCTION 86

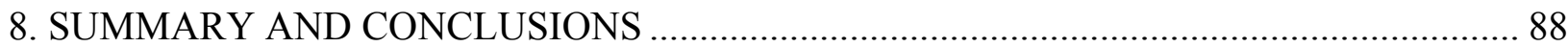

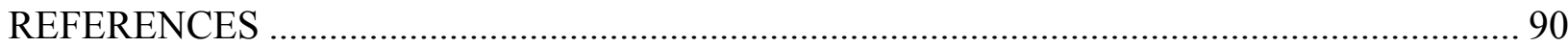

APPENDIX: MCMC SENSITIVITY STUDIES USING MILROW MODEL ………………..... 95

\section{LIST OF FIGURES}

2.1. Location of model cross section for each site with the cartoon eye indicating the perspective of subsequent figures. .................................................................................... 4

2.2. Milrow profile that determines the upper boundary of the simulation domain (a), and the discretization and boundary conditions (b)...................................................... 5

4.1. Head data used as calibration targets in the 2002 model of Hassan et al ........................ 17

4.2. Salinity data used as calibration targets in the 2002 model of Hassan et al ..................... 20

4.3. Resistivity profiles as obtained from the inversion models used in conjunction with

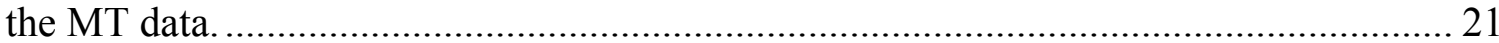

4.4. Variation of total porosity determined from laboratory tests on core samples................. 22

4.5. Effective porosity profiles provided by Unsworth et al. (2005) for the three sites $(a, b$, and $c)$, and scaled down profiles to enforce a maximum porosity of 0.4 at the

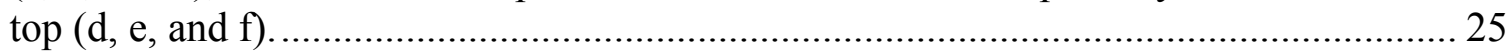

4.6. Two-dimensional map of bathymetric levels provided by Johnson and Stewart (2005) (a) and comparison between bathymetric profiles used in 2002 model and those extracted from the top map for Cannikin (b) and Long Shot (c)........................... 27

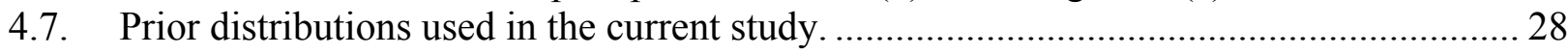

4.8. A flowchart showing the steps of the MCMC algorithm implemented in this study....... 32

4.9. A schematic showing the selection of the initial value, $K^{0}$, from the prior distribution, and the construction of the proposal density with the initial value as its mean................. 33

4.10. Relationship between $R / K$ and the sum of squared errors (a), and the error variance (b) used in the likelihood function for Milrow................................................... 34

4.11. Relationship between $R / K$ and the sum of squared errors (a), and the error variance (b) used in the likelihood function for Long Shot............................................. 35

4.12. Relationship between $R / K$ and the sum of squared errors (a), and the error variance (b) used in the likelihood function for Cannikin.

4.13. Milrow MCMC diagnostics for $R$ : (a) parameter trace, (b) posterior mean trace, (c) posterior variance trace, and (d) histogram of $\log (R)$ with a fitted posterior frequency curve (red line)

4.14. Milrow MCMC diagnostics for $K$ : (a) parameter trace, (b) posterior mean trace, (c) posterior variance trace, and (d) histogram of $\log (K)$ with a fitted posterior frequency curve (red line).

4.15. Milrow MCMC diagnostics for $R / K$ : (a) parameter trace, (b) posterior mean trace, (c) posterior variance trace, and (d) histogram of $\log (R / K)$ with a fitted posterior frequency curve (red line).

4.16. Joint posterior density of $R$ and $K$ (a), and the parameter trace plot (b) showing the strong positive correlation.

4.17. Resistivity map and interpreted transition zone boundaries (Lines A and B) from Unsworth et al. (2005) with the salinity profile from UAe-2 at Milrow (red line) superimposed. 
4.18. First and second options for converting MT to concentration data at Milrow............... 44

4.19. Third and fourth options for converting MT to concentration data at Milrow....

4.20. Results of the sequential conditioning approach for Milrow where $D_{1}$ is the concentration data set (salinity at UAe-2 and MT-converted concentrations) and $D_{2}$ is the head data at UAe-2.

4.21. Recharge-conductivity pairs (a) as used in the Milrow 2002 model, (b) generated from the MCMC algorithm, and (c) extracted from the MCMC results for use in the revised model.

4.22. Results of the sequential conditioning approach for Long Shot where $D_{1}$ is the concentration data set (salinity at EH-5 and MT-converted concentrations) and $D_{2}$ is the head data at a number of wells.

4.23. Recharge-conductivity pairs (a) as used in the Long Shot 2002 model, (b) generated from the MCMC algorithm, and (c) extracted from the MCMC results for use in the revised model.

4.24. Results of the sequential conditioning approach for Cannikin where $D_{1}$ is the concentration data set (salinity at UAe-1 and MT-converted concentrations) and $D_{2}$ is the head data at UAe-1, UA-1, and HTH-1

4.25. Recharge-conductivity pairs (a) as used in the Cannikin 2002 model, (b) generated from the MCMC algorithm, and (c) extracted from the MCMC results for use in the revised model.

5.1. Expanded view of the upper left portion of each model domain, showing the nuclear chimney included in the flow and transport simulations for Milrow (A),

Long Shot (B), and Cannikin (C).... 58

5.2. Geometry of simulation domain and boundary conditions for the flow problem........... 61

5.3. Comparison between the distributions of Milrow parameters used in Hassan et al. (2002) and the distributions used in this study based on new data

5.4. Mean \pm one standard deviation for salinity and head profiles at UAe-2 location at Milrow as obtained from the new model and the 2002 model.

5.5. Comparison between the distributions of Long Shot parameters used in Hassan et al. (2002) and the distributions used in this study based on new data...

5.6. Mean \pm one standard deviation for salinity profile at EH-5 and head profile (at a cell where multiple wells are clustered) at Long Shot as obtained from the new model and the 2002 model.

5.7. Comparison between the distributions of Cannikin parameters used in Hassan et al. (2002) and the distributions used in this study that are based on new data.

5.8. Mean \pm one standard deviation for salinity and head profiles at UAe-1 location at Cannikin as obtained from the new model and the 2002 model.

5.9. Mean \pm one standard deviation for salinity profiles at UA-1 (a) and at HTH-1 (b) at Cannikin as obtained from the new model and the 2002 model 70

6.1. Flow chart of the transport model source and processes considered in the 2002 study... 71

6.2. Particle positions at 2,200 years from two realizations: a) a 2002 model realization with the highest porosity (i.e., slowest realization), and b) a current model realization with the same $R / K$ and with the lower porosity bound shown in Figure 4.5 ................. 74

6.3. Mass flux moments as computed with only advection and dispersion........................... 75 
6.4 Milrow domain and mesh configuration showing the cavity and chimney location and the cross section A-A at which fluxes and velocities are computed for next two figures.

6.5. Distribution of Milrow groundwater fluxes at section A-A shown in Figure 6.4 and for all realizations. Horizontal flux (a), vertical flux (b) and magnitude of the flux vector (c) are shown.

6.6. Distribution of Milrow groundwater velocities at section A-A shown in Figure 6.4 and for all realizations. 78

6.7 Long Shot domain and mesh configuration showing the cavity and chimney location and the cross section B-B at which fluxes and velocities are computed for next two figures. 80

6.8. Distribution of Long Shot groundwater fluxes at section B-B shown in Figure 6.7 and for all realizations.

6.9. Distribution of Long Shot groundwater velocities at section B-B shown in Figure 6.7 and for all realizations.

6.10. Cannikin domain and mesh configuration showing the cavity and chimney location and the cross section $\mathrm{C}-\mathrm{C}$ at which fluxes and velocities are computed for next two figures.

6.11. Distribution of Cannikin groundwater fluxes at section C-C shown in Figure 6.10 and for all realizations.

6.12. Distribution of Cannikin groundwater velocities at section $\mathrm{C}-\mathrm{C}$ shown in Figure 6.10 and for all realizations.

\section{LIST OF TABLES}

4.1. Hydraulic head calibration targets used in Hassan et al. (2002)................................... 16

4.2. Groundwater chemistry calibration targets used in Hassan et al. (2002)....................... 19

4.3. Porosity distributions used to generate realizations for each model reported in Hassan et al. (2002).

4.4. MCMC diagnostic analysis of the effect of the number of burn-in iterations and the total number of iterations on the mean and variance of generated parameters................ 40

4.5. Summary of MCMC results showing percent reduction in variance............................ 57

5.1. Parameters used in FEFLOW to solve the density-driven flow problem ....................... 60

5.2. Parameter range for Milrow flow and transport simulations................................... 63

5.3. Parameter range for Long Shot flow and transport simulations. ................................. 65

5.4. Parameter range for Cannikin flow and transport simulations. .................................. 68

\section{LIST OF ACRONYMS}

CRESP Consortium for Risk Evaluation and Stakeholder Participation

DOE U.S. Department of Energy

MCMC Markov Chain Monte Carlo

MT magnetotelluric

RFG random field generator

TZ transition zone

USGS U.S. Geological Survey 


\section{INTRODUCTION}

The United States conducted nuclear testing in the 1960s and early 1970s on Amchitka Island, in the Aleutian Island chain off western Alaska, to characterize the seismic signals from underground tests in active tectonic regimes, and to avoid proximity to high-rise buildings and resulting ground-motion problems from high-yield tests. The tests were named Long Shot, Milrow, and Cannikin, and were detonated on October 29, 1965, October 2, 1969, and November 6, 1971, respectively. The announced yield of these tests are 80 kilotons for Long Shot, approximately one megaton for Milrow, and less than five megatons for Cannikin (DOE, 2000). As the U.S. Department of Energy focused on environmental management of nuclear sites in the 1990s, a decision was made to revisit contaminant transport predictions for the island, taking advantage of the advances in the understanding of island hydraulic systems and in computational power that occurred in the decades after the tests.

The groundwater flow and radionuclide transport at the Amchitka Island underground nuclear tests were modeled using two-dimensional numerical simulations as described in Hassan et al. (2002). The flow model was conceptualized to address the problem of densitydriven flow encountered in the saltwater intrusion environment. A multi-parameter uncertainty analysis was adapted and used to address the effects of the uncertainties associated with the definition of the modeled processes and the values of the parameters governing these processes. Groundwater flow and transport were modeled in a stochastic framework. A calibration was performed for each test location using head data and groundwater chemistry data from nearby boreholes as calibration targets. Simultaneous, exact matches of these two independent data sets were not achieved at any site, though the uncertainty expressed by the standard deviation in the base-case flow models encompassed the observed data. Results were presented as expected values and uncertainties of radionuclide mass flux leaving the groundwater system and discharging into the bottom of the ocean.

After the groundwater modeling was complete, the U.S. Department of Energy and the State of Alaska Department of Environmental Conservation committed by a Letter of Intent to a program to achieve closure and long-term stewardship of Amchitka. One focus of the program was model verification and reduction of risk uncertainty. The Consortium for Risk Evaluation and Stakeholder Participation (CRESP) developed an Amchitka Independent Assessment Science Plan (CRESP, 2003) to establish the framework for a scientific assessment providing a basis for long-term stewardship. This led to a field expedition during summer 2004, the results of which were published the following year (CRESP, 2005).

Several of the CRESP field activities were directed at providing data related to groundwater flow at Amchitka. These include:

- oceanographic investigations of bathymetry

- oceanographic investigations of discharge of freshwater through the ocean floor

- geophysical (magnetotelluric or MT) measurements for determining the subsurface salinity and porosity structure of Amchitka 
In addition to the MT and bathymetric data collection activities, CRESP also supported a groundwater modeling effort. Forsstrom and Barnes (2005) constructed a twodimensional flow model through the Long Shot site. Their model differs from that of Hassan et al. (2002) in two significant ways. First, Forsstrom and Barnes (2005) modeled flow in a full transect across the island, allowing the model to establish the location of the groundwater divide, while Hassan et al. used an island half-width assumption, specifying the groundwater divide as a boundary condition (the impacts of this assumption were tested by sensitivity analysis). Second, Forsstrom and Barnes explicitly modeled an andesite sill layer as a preferential flow pathway, while Hassan et al. did not include the andesite sill as a deterministic feature due to its unknown continuity and extent, but rather tested the impact of continuous length conductive features through sensitivity analysis. These scenarios are not suggested by newly collected data, but rather represent alternate conceptualizations or implementations of the Long Shot flow model. This report focuses on verification and uncertainty reduction of the 2002 model based on the newly collected data.

The new data sets did not contain definitive observations of freshwater discharge at the seafloor to aid in model verification and uncertainty reduction. The new bathymetric data are compared to the previous bathymetry used in the 2002 model. Of most importance to the groundwater model are the magnetotelluric (MT) measurements for determining the subsurface salinity and porosity structure of the Island (Unsworth et al., 2005). During the 2004 CRESP Amchitka Island Expedition, magnetotelluric data were collected on profiles that passed through each of the three test sites. The data presented in Unsworth et al. (2005) showed a pattern of increasing, decreasing, and increasing resistivity at each test site on Amchitka. The inflection point of the resistivity profile where resistivity begins to decrease is interpreted as the top of the transition zone (TZ) where the salinity increases. The deeper inflection point (increase in resistivity after its decrease with depth) is interpreted as the base of the transition zone, where salinity remains constant and porosity decreases (Unsworth et al., 2005).

This independent set of data provides an opportunity for verifying the groundwater model at the three sites and reducing the uncertainty in the model parameters and subsequently the model output. To accomplish this, data pertaining to the location of the freshwater lens (from the interpretation of the MT data) can be compared to the groundwater model input distributions for verification, and then the distributions tightened around the new data for uncertainty reduction. The original groundwater flow and transport models for Amchitka incorporated uncertain distributions for a number of parameters with the distributions centered on best-estimate values for these parameters. These values were based on field evidence, calibration to available data of dependent parameters, or literature values. These best-estimate values for some parameters can be improved based on the CRESPcollected data. These data are combined with the original data available for the three Amchitka sites (Milrow, Cannikin, and Long Shot). For example, the determination of the best-estimate value for the recharge-conductivity ratio at Milrow relied heavily on the thickness of the freshwater lens identified from the salinity profile available from borehole UAe-2. Given that the new data indicate a different thickness at Milrow, the mean value for the recharge-conductivity ratio can be adjusted accordingly. Finally, additional data allows the uncertainty distributions in the original model to be tightened. 
Interest in the assessment of parameter uncertainty in hydrologic models and advances in computing technology have led to the use of Monte Carlo-based methods. These include the generalized likelihood uncertainty estimation (GLUE) procedure, simple uniform random sampling (URS) over the feasible parameter space, and Markov Chain Monte Carlo (MCMC) methods. Although the GLUE and URS methods facilitate a detailed exploration of the parameter space, they are computationally inefficient, as they rely on very large sample sizes. In contrast, MCMC simulation generates a sample of parameter values from a carefully constructed Markov chain that converges to a stationary distribution. This distribution can be used to summarize parameter uncertainty accurately and quantify its effects on model predictions. One of the limitations of the GLUE methodology is that the selection of the weighting factors is somewhat arbitrary and there is no commonly accepted rule for selecting the exponent of the function defining the weights. The MCMC method provides a rigorous alternative for solving the inverse problem (calibration problem) in a Bayesian framework that benefits from prior knowledge as well as newly collected data.

This report presents the verification analysis and the use of the MCMC method to develop, quantify, and constrain the uncertainty in model parameters using all available information. It also presents a new set of flow and transport results that are conditioned on the newly collected data on the island. The remainder of the report is organized as follows. Section 2 presents a brief review of the original groundwater flow and transport model (Hassan et al., 2002), highlighting the key features of the model, the modeling approach, and the original results. In Section 3, a detailed theoretical background about Bayesian statistical inference and the MCMC approach is presented. Section 4 presents the MCMC analysis for Milrow, Long Shot, and Cannikin, and Sections 5 and 6 present the updated flow and transport results, respectively, based on new distributions (posterior distributions) generated in Section 4. Section 7 then presents a summary of the key aspects of model verification and the achieved uncertainty reduction in model input as well as output. Section 8 summaries the work and the main conclusions drawn from the analysis and results. Detailed MCMC studies and sensitivities are presented in the Appendix.

\section{OVERVIEW OF THE ORIGINAL GROUNDWATER MODEL}

Amchitka is a long, thin island separating the Bering Sea and Pacific Ocean, predominantly consisting of Tertiary-age submarine and subaerially deposited volcanic rocks. The nuclear tests all occurred in the lowland plateau region of the island, where the lithologic sequence is dominated by interbedded basalts and breccias. Though the general geologic conditions are similar for the three tests, they differ in their depth and thus position relative to the freshwater-seawater TZ. The shallowest test was Long Shot, at a depth of 700 $\mathrm{m}$. The Milrow test occurred at a depth of 1,220 m. The deepest test was Cannikin at 1,790 m.

There are strongly developed joint and fault systems on Amchitka and groundwater is believed to move predominantly by fracture flow between matrix blocks of relatively high porosity. The subsurface is saturated to within a couple of meters of ground surface, and the lowland plateau has many lakes, ponds, and streams. Data show that hydraulic head decreases with increasing depth through the freshwater lens, supporting the basic conceptualization of freshwater recharge across the island surface with downward-directed gradients to the transition zone above seawater. Samples of groundwater from exploratory 
boreholes at each site indicate that Long Shot was detonated in the freshwater lens and Milrow was below the TZ. The data from Cannikin are equivocal, and though Cannikin is deeper than Milrow, the possibility of asymmetry in the freshwater lens precludes extrapolation. In addition to chemical data from wells and boreholes, numerous packer tests were performed and provide hydraulic data, and abundant cores were collected and analyzed for transport properties (such as porosity and sorption).

\subsection{Conceptual Models}

The conceptual model of flow for each site is governed by the principles of island hydraulics. Recharge of precipitation on the ground surface maintains a freshwater lens by active circulation downward and outward to discharge on the sea floor. Below the TZ there is a very low velocity counter-circulation in the salt-water zone, driven by the loss of salt dispersed into the $\mathrm{TZ}$ and recharged by infiltration along the sea floor far beyond the beach margin, past the freshwater discharge zone. A groundwater divide is assumed to exist, coincident with the topographic divide, separating flow to the Bering Sea (applicable for Long Shot and Cannikin) from flow to the Pacific Ocean (Milrow). The simplicity of the island hydraulic model is enhanced by the absence of pumping or any form of groundwater development on the island, so that steady-state conditions are assumed. Figure 2.1 shows a map of Amchitka Island and the location and perspective of each of the three cross sections representing the simulation domains for the three tests.

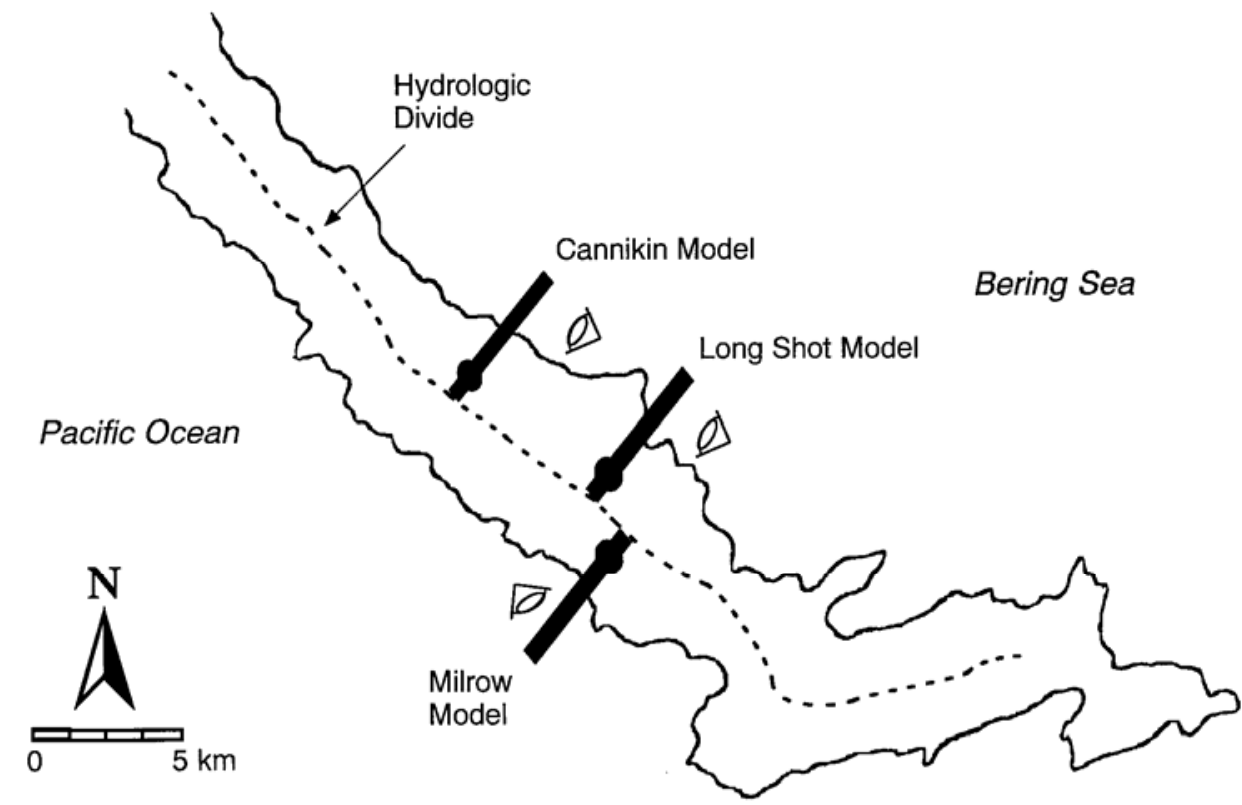

Figure 2.1. Location of model cross section for each site with the cartoon eye indicating the perspective of subsequent figures.

The symmetry of island hydraulics lends itself to considering flow in two dimensions, on a transect from the hydrologic divide along the island's centerline, through the nuclear test 
location, and on to the sea. The boundary conditions for the flow problem entail no flow coinciding with the groundwater divide and along the bottom boundary. The seaward boundary is defined by specified head and constant concentration equivalent to seawater. The top boundary has two segments. The portion across the island receives a recharge flux at a freshwater concentration, and the portion along the ocean is a specified head dependent on the bathymetry. Figure 2.2 shows the Milrow topographic and bathymetric profile, the domain geometry and boundary conditions, and the finite-element mesh used to discretize the density driven flow equations. The mesh is refined in the entire left upper triangle of the simulation domain since the $\mathrm{TZ}$ varies widely with the parameters selected from their distributions.

For the Long Shot and Cannikin sites, similar domain geometry and boundary conditions are utilized. However, the upper boundary is determined based on the specific site's topography and bathymetry, which is slightly different among the three sites. The dimensions, domain geometry, and discretization for Long Shot and Cannikin are presented in Hassan et al. (2002).
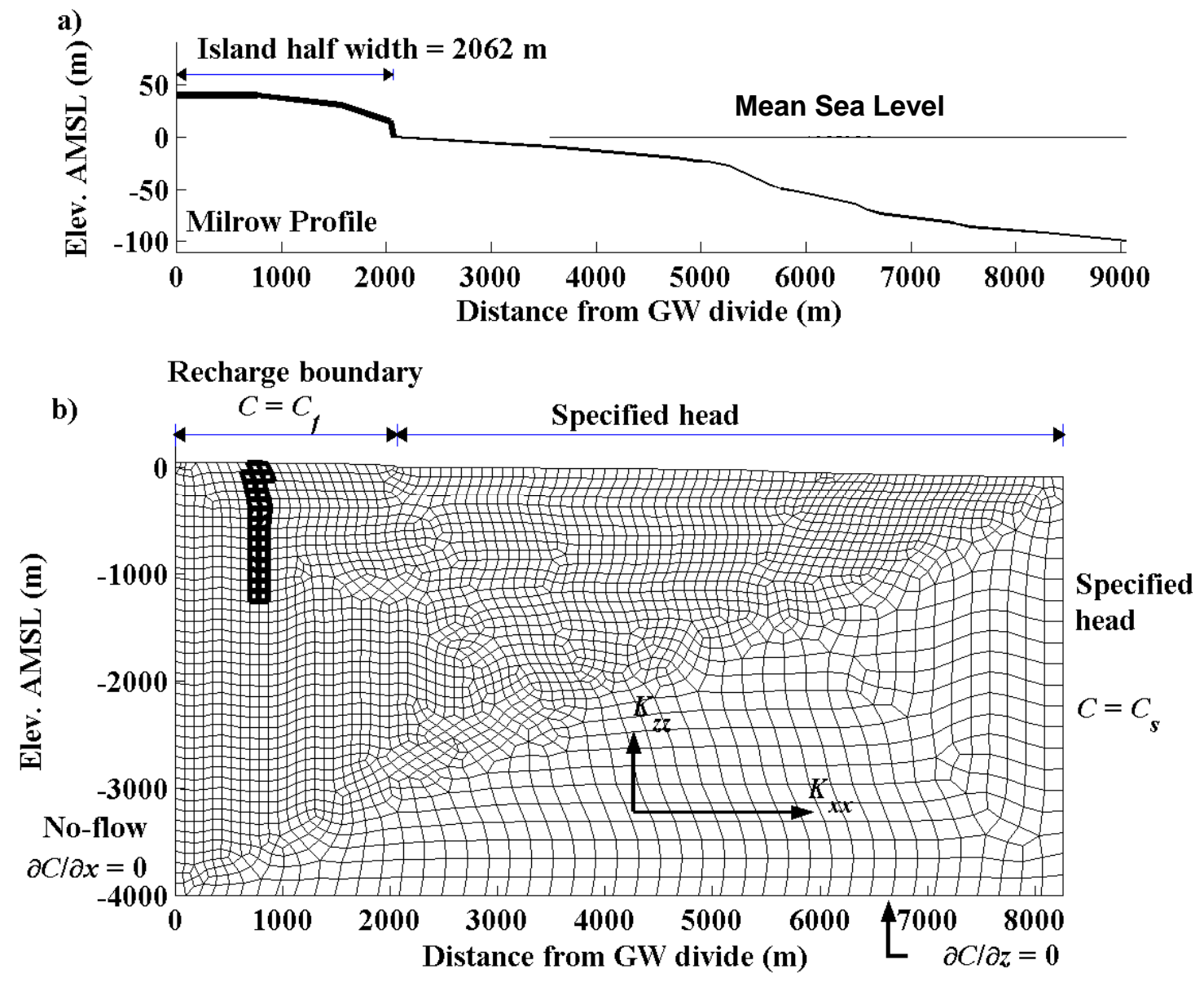

Figure 2.2. Milrow profile that determines the upper boundary of the simulation domain (a), and the discretization and boundary conditions (b). 


\subsection{Processes Modeled, Parameters, and Calibration}

Modeling the fate of radionuclides emplaced during nuclear tests on Amchitka encompasses two major processes: 1) the flow modeling, taken here to include density driven flow and saltwater intrusion, and 2) the contaminant transport modeling, combining radioactive source evaluation and decay, retardation processes, release functions, and matrix diffusion.

Island-specific data are used to constrain the parameter values used to construct the seawater intrusion flow problem. Hydraulic conductivity $(K)$ data collected from six boreholes are used to calculate the best estimate for a homogeneous conductivity value and the range of uncertainty associated with this estimate. The geologic environment suggests strong anisotropy, so that vertical hydraulic conductivity, $K_{z z}$, is assumed to be one-tenth the horizontal value (except in the chimney above the nuclear cavity, where collapse is assumed to increase $K_{z z}$ relative to that of the horizontal conductivity, $K_{x x}$ ). Temperature logs measured in several boreholes and water balance estimates are used to derive groundwater recharge $(R)$ values. Measurements of total porosity on almost 200 core samples from four boreholes provided a mean and distribution for matrix porosity. A wide range of values have been estimated for fracture porosity, a notoriously difficult-to-measure parameter, with the mean and distribution used in the models conservatively favoring smaller values. The transport model also required data on retardation properties, which were obtained from sorption and diffusion experiments using core material.

The model for each of the nuclear tests was calibrated using site-specific hydraulic head and water chemistry data. The objective of the calibration was to select base-case, uniform flow, and saltwater intrusion parameters that yield a modeling result as close as possible to that observed in the natural system. Difficulty was encountered in obtaining simultaneous best fit to the two targets (head and chemistry). The best parameters to match head would result in a less perfect match for the chemical profile, and vice versa. The critical calibration feature for locating the mid-point of the $\mathrm{TZ}$ is the ratio of recharge to hydraulic conductivity $(R / K)$. Macrodispersivity controlled the width of the TZ modeled around the mid-point. Ultimately, compromises were made to achieve the optimum fit to both heads and chemistry, and more weight was given to the hydraulic head measurements due to reported difficulties encountered in obtaining representative water samples from these very deep boreholes during drilling operations. The configuration of the seawater interface differs from one site model to another, with a deeper freshwater lens calculated on the Bering Sea side of the island.

\subsection{Uncertainty Analysis}

To optimize the modeling process, a parametric uncertainty analysis was performed to identify which parameters are important to treat as uncertain in the flow and transport modeling and which to set as constant, best estimate, values. The processes evaluated include recharge, saltwater intrusion, radionuclide transport, glass dissolution, and matrix diffusion. The analysis compared the effect of uncertainty of each parameter on the modeled arrival time and mass flux of radionuclides crossing the seafloor.

Uncertainty in $K, R$, fracture porosity $(n)$, and matrix diffusion parameter $(\kappa)$, had the strongest impact on transport predictions. Multiple realizations of the flow field, with varying $K, R$, and $n$, were generated to evaluate the effect of uncertainty in these parameters on the 
flow and transport results. The impact of uncertainty in $\kappa$, a transport parameter, was evaluated during sensitivity analysis of the transport model.

The primary flow and transport modeling for the sites used the significant uncertain parameters identified in the parametric uncertainty analysis $(K, R$, and $n)$. Three random distributions were generated for $K, R$, and $n$ for each site with 300 realizations for Milrow, 240 realizations for Long Shot, and 260 realizations for Cannikin. Head and chloride concentration data were used as criteria to ensure that the combined random distributions lead to realistic flow solutions. The output of this analysis was the expected value and uncertainty of total mass flux discharging into the ocean for each individual radionuclide taken from an unclassified source term, using a unit starting mass. The detailed analysis and results of the groundwater model can be found in Hassan et al. (2002) .The unit-mass-based breakthrough curves were scaled with the classified source values to generate actual mass breakthrough (G. Pohll, Desert Research Institute, personnel communication, 2003).

\section{BAYESIAN MARKOV CHAIN MONTE CARLO METHOD: THEORETICAL BACKGROUND}

A major difficulty in accurately describing subsurface flow and transport processes arises from the uncertainty that is typical of groundwater problems. Uncertainty stems from a number of factors including parametric uncertainty, spatial variability, conceptual uncertainty, and boundary uncertainty. Data scarcity is the major factor that leads to the great level of uncertainty about subsurface parameters. Typically, groundwater flow and transport models account for the different types of uncertainties using both analytical and numerical tools. These tools provide a means for propagating the different uncertainty attributes through the modeling protocol to the final output of the model.

\subsection{The Inverse Problem (Calibration Problem)}

How to obtain appropriate parameters for any modeling study has long been a subject of debate within the hydrologic community (Marshall et al., 2004). In groundwater studies, one is commonly faced with the inverse problem: the distribution of the dependent variable (e.g., head) is the most well known aspect of the system, whereas the distribution of the independent variable (e.g., hydraulic conductivity) is the least well known. Model calibration is the process used to solve this inverse problem. That is, model calibration is the process of tuning the model to identify the independent input parameters by fitting the model results to field or experimental data, representing the dependent system parameters. The calibration process can be quantitatively described by the goodness of fit. However, in many cases, the calibration process is performed on a qualitative basis and the deviation between model prediction and field data (calibration data) is minimized based mainly on visual inspection.

Traditionally, model calibration in groundwater relied on manual trial-and-error adjustment of the model independent parameters (e.g., hydraulic conductivity, recharge, boundary conditions) until a good match between modeled and observed dependent parameters (e.g., heads) occurs. The judgment of a good versus a bad match was based on visual inspection. In recent years, a great deal of research has focused on developing automated calibration techniques that rely on numerical optimization methods. This was driven by the fact that prediction reliability of groundwater models was difficult to assess using only a nonautomated calibration procedure (Poeter and Hill, 1997). A number of 
approaches for the solution of the automated inverse problem has been developed including nonlinear least-squares regression (Cooley 1982a,b; Doherty, 1990, 1994; Hill, 1992; Poeter and Hill, 1997) geostatistical approaches (Hoeksema and Kitanidis 1984; Zimmerman et al. 1998), L1 norm-based parameter estimation technique (Xiang et al. 1992), and automated pilot point approach (LaVenue et al., 1995; RamaRao et al., 1995), just to name a few.

Automatic calibration provides a number of benefits and advantages over traditional nonautomated, trial-and-error-based calibration (Poeter and Hill, 1997). First, it is much faster, allowing more time to be spent on changing the conceptual model. Second, automated calibration can yield quantitative evaluation of the quality of calibration, and the associated confidence in the parameter estimates. Third, automated calibration can reveal issues that may otherwise be overlooked such as parameter correlation or lack of sensitivity of parameter values to calibration data (Poeter and Hill, 1997).

While automated calibration is superior to manual calibration, both approaches lack the ability to rigorously assess the uncertainty in calibrated parameters. In hydrologic modeling, the uncertainty of parameter estimation (or calibrated parameters) needs to be accounted for and the impact on the model output uncertainty needs to be quantified. Bayesian inference provides a framework within which these issues can be addressed, with the end product of models being a probability distribution known as the posterior distribution of the model parameters (input) and predictions (output) quantifying uncertainty after data have been collected and incorporated.

\subsection{Bayesian Statistical Inference}

Bayesian methods are an alternative to the classical approach to statistical analysis. Such methods are used more frequently, largely due to the advances in computer power and the development of user-friendly software. Bayesian methods allow the incorporation of information external to the study into the analysis. Such information is specified in a prior distribution and is combined with the study data to produce a posterior distribution on which statistical inferences about the system's dependent parameters are based.

Bayesian inference has similarities to maximum likelihood, but the basic question one asks is completely different. In maximum likelihood, the question is 'What is the probability of seeing the observed data $(D)$ given that a certain model/assumption $(M)$ is true, $P(D \mid M)$ ?', whereas the question in Bayesian inference is 'What is the probability that this model/assumption $(M)$ is correct, given the data $(D)$ that have been observed, $P(M \mid D)$ ?' The latter question is answered by Bayes' theorem:

$$
P(M \mid D)=\frac{P(M) P(D \mid M)}{P(D)}
$$

where $P(M \mid D)$ is the posterior probability, $P(M)$ is the prior probability, that is the probability assigned to the theory or model $M$ before the data were collected, $P(D \mid M)$ is the likelihood, and $P(D)$ is the probability of observing $D$.

Bayesian methods allow pre-existing knowledge about the parameters of a model to be combined with observed data and the model output. This results in a probability distribution on the parameter space (called the posterior distribution) that summarizes uncertainty about the parameters (Marshall et al., 2004). It is important to note that the 
incorporation of subjective/ informative prior beliefs is not a requirement of a Bayesian analysis, because "vague" or "noninformative" priors can be used that convey very little or no information relative to the data.

Although a number of recent studies used the Bayesian framework for rainfall-runoff modeling and parameter estimation (e.g., Kuczera, 1983; Freer et al., 1996; Kuczera and Parent, 1998; Kuczera and Mroczkowski, 1998; Bates and Campbell, 2001; Marshall et al., 2004), its application to other areas has been limited due to computational difficulties. The advent of Markov Chain Monte Carlo (MCMC) methods has helped address some of these computational difficulties (Marshall et al., 2004).

For certain simple analyses, the posterior distributions (on which inferences are usually made) are analytically tractable, meaning that they can be written down in standard statistical notation. When this is not possible, a Bayesian solution can still be obtained through the use of simulation methods, such as the MCMC methods discussed below. Some details about the Bayesian framework used with the groundwater model at Amchitka are presented next.

For the stochastic groundwater flow model at Amchitka it is assumed that the newly collected data are expressed by the data vector $\mathbf{D}$. One of the elements in this data vector is be the groundwater salinity profile beneath the island, $C$, and another is the head data, $h$, both of which represent the steady-state output of the model. One can express the model as

$$
\mathbf{D}(\mathbf{x})=M(\mathbf{G}, \mathbf{x} ; \boldsymbol{\Theta})+\varepsilon(\mathbf{x})
$$

where $\mathbf{D}(\mathbf{x})$ is the observed data at location $\mathbf{x}, M(\mathbf{G}, \mathbf{x} ; \Theta)$ is the model output for location $\mathbf{x}$, $\mathbf{G}$ is the set of model input describing domain geometry, boundary conditions, and discretization, $\Theta$ is the vector of unknown model parameters to be estimated from the data (e.g., hydraulic conductivity, recharge, porosity), and $\varepsilon(\mathbf{x})$ is an error term that is assumed to be a normally distributed random variable having mean 0 and variance $\sigma_{\varepsilon}^{2}$.

The set of model input $\mathbf{G}$ includes those aspects that do not change from one realization to another. The set of model parameters $\Theta$ includes all the uncertain parameters that are needed to run the model and that change from one model realization to another. The vector $\Theta$ is treated as a random variable distributed according to a probability density function. This density function expresses the uncertainty about $\Theta$. Before considering the newly collected data, the knowledge about the model parameter set can be summarized in a distribution $P(\Theta)$ called the prior distribution.

The decision to use a particular prior should be based on any available knowledge about the parameter (Marshall et al., 2004). This may include comparing the function of the parameter within the model to those of established models. Estimated optimum values of parameters from previous studies may be useful for establishing the prior, and constraints on the model parameters should also be taken into account. The posterior distribution of the parameter set, $P(\Theta \mid \mathbf{D})$, can be obtained through the application of Bayes' theorem

$$
P(\Theta \mid \mathbf{D})=\frac{P(\Theta) P(\mathbf{D} \mid \Theta)}{P(\mathbf{D})}
$$


where $P(\mathbf{D} \mid \Theta)$ describes the likelihood of the newly collected data given that the model parameters were correctly chosen and $P(\mathbf{D})$ is a proportionality constant assuring that $P(\Theta \mid$ D) is a proper density function. It is important to note that the posterior distribution assumes a shape similar to the prior when available data are limited. But when there are large data sets, the posterior distribution will be influenced more by the data than by the assumed prior distribution. Also, the information in the new sample data will dominate the posterior if the prior distribution is selected to represent vague prior knowledge. $P(\Theta \mid \mathbf{D})$ thus contains all of the available information about $\Theta$ coming from both prior knowledge and collected data. Thus Bayesian inference reduces to summarizing the posterior density $P(\Theta \mid \mathbf{D})$.

One of the advantages of the Bayesian estimation is that it provides a formal mechanism for combining prior information based on historical data or expert knowledge (through the prior distribution) and data collected by observation. Despite this advantage, the application of this Bayesian estimation technique in hydrogeologic studies was hindered mainly due to computational difficulties. The difficulty stems from the fact that computing the posterior distribution parameters is often complex, requiring the evaluation of numerous high-dimensional integrals that have no closed-form solution. The MCMC sampling is a relatively new development in the Bayesian statistical literature and provides a simple and elegant way around the computational difficulties noted above (Marshall et al., 2004). This sampling generates a random process (a Markov process) that eventually converges to the stationary, posterior distribution of the parameters.

\subsection{Markov Chain Monte Carlo}

As stated above, Bayesian evaluation of parameter uncertainty in stochastic models is hindered by the fact that no analytical solution exists for the posterior distribution. However, the Markov Chain Monte Carlo method can be used to simulate the posterior distribution. This method is a multidimensional, random sampling procedure where the parameter value of the next sample only depends on the parameter value of the current sampling point. In $\mathrm{MCMC}$, the posterior distributions of model parameters are generated by drawing several thousands of samples from the posterior distributions, and calculating their empirical mean, standard deviation, and percentiles, similar to simple Monte Carlo simulation, except that Monte Carlo draws samples from what is, in effect, a prior distribution, and does not update this with likelihood.

While there exist many different MCMC sampling algorithms, the MetropolisHastings algorithm is the most commonly used. The Metropolis-Hastings algorithm produces a Markov chain sequence of samples that constitute a random walk in the parameter space. Each iteration of the algorithm generates proposed (or candidate) parameter samples using a suitable arbitrary probability distribution referred to as the proposal density or the jump distribution. These proposed values are either accepted or rejected using a criterion, which ensures the algorithm is sampling from the posterior distribution when the Markov chain has become stationary.

The application of the Metropolis-Hastings algorithm for drawing samples from the posterior distribution can be summarized as follows (Marshall et al., 2004):

1) Start the simulation at iteration $i=0$ with an arbitrarily chosen initial parameter vector $\Theta=\Theta^{0}$. 
2) Generate a proposed value $\Theta$ * for $\Theta$ from a proposal density depending on the current value $\boldsymbol{\Theta}^{i}$ of $\Theta$.

3) Compute an acceptance probability $\alpha$ (depending on $\Theta^{i}, \Theta^{*}$, the proposal density, and the model) that determines whether the proposed value $\Theta^{*}$ is accepted or not.

4) Accept $\Theta^{i+1}=\Theta^{*}$ with probability $\alpha$. Otherwise $\Theta^{i+1}=\Theta^{i}$.

5) Perform an increment iteration $i$ and repeat steps 2 through 4.

The difficult step of evaluating the moments and other statistics of the posterior distribution in Bayesian inference is circumvented in this algorithm, as one can get a reliable estimate of these moments once a large sample of parameter values has been generated. This assumes that the sample moments converge to the moments of the distribution, and that for a given starting value $\Theta^{0}$ and a given proposal density, the parameter sequence $\Theta^{i}$ has converged in distribution to the stationary posterior distribution (Smith and Roberts, 1993; Campbell et al., 1999). This can be achieved by discarding the first few thousand "burn in" simulations, or samples that are influenced by the initial value.

It is important to note that the variance (i.e., the spread) of the proposal density influences how far from the current state the proposed state is likely to be and its probability of being accepted. If this variance is too small, the iterative process will induce a Markov chain that does not mix rapidly enough over the parameter space. As a result, the tail regions of the posterior may not be sampled sufficiently. If the variance is too large, proposed values will be frequently rejected. It follows that the acceptance rate of the algorithm can be used as a measure of efficiency. An acceptance rate between 30 and 70 percent appears to work well (Bates and Campbell, 2001). Reasonable acceptance rates are often achieved by using proposal densities with variances about the same or somewhat larger than the marginal posterior variances. The proposal variances are determined from tuning runs starting with arbitrary values (Weir, 1997; Campbell et al., 1999), and they can also be tuned during the iterations of the Metropolis-Hastings algorithm to ensure the acceptance rate remains within a suitable range.

The MCMC scheme uses the Metropolis-Hastings algorithm with either a single-site or a block updating. In single-site updating, candidate values for each component of $\Theta$ are generated in turn. A candidate value $\theta_{i}^{\prime}$ from a univariate density $q_{i}\left(\Theta, \theta_{i}^{\prime}\right)$ is proposed that depends on the current value, $\Theta$. The move from the current state $\Theta=\left(\theta_{1}, \ldots, \theta_{i}, \ldots, \theta_{N}\right)$ to the proposed state $\Theta^{\prime}=\left(\theta_{1}, \ldots, \theta^{\prime}, \ldots, \theta_{N}\right)$ is accepted with probability

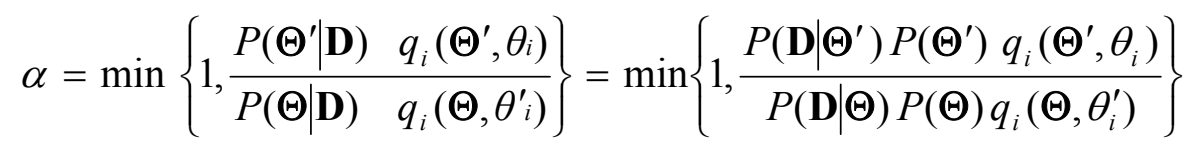

Block updating, which involves the generation of candidate values for two or more parameters at a time using multivariate proposal densities, is important when dealing with highly correlated parameters. The Metropolis-Hastings algorithm converges to $P(\Theta \mid \mathbf{D})$ provided the constructed Markov chain is irreducible (i.e., it is possible for the algorithm to go from any point in the parameter space to any other point given enough steps) and 
aperiodic (i.e., the number of steps until the chain returns to its current state is not a multiple of an integer > 1) (Smith and Roberts, 1993; Tierney, 1994).

The MCMC methods are implemented using either an independent chain or a random walk chain. An independent chain proposes using candidate states from the same probability distribution at each step, regardless of the current state. A random walk chain uses the current state as the mean for the candidate proposal density. The random walk chains are usually preferred, as they give the method freedom to locate regions of high probability in a highdimensional parameter space and to explore them.

In the context of conceptual rainfall-runoff modeling, Marshall et al. (2004) compared four MCMC algorithms including 1) a conventional Metropolis-Hastings algorithm commonly used in hydrological applications, which uses a combination of block and single-site updating, 2) an adaptive Metroplois algorithm with characteristics that are suited to model parameters with a high degree of correlation and interdependence, 3 ) a Metropolis algorithm with single-site updating, and 4) a Metroplois algorithm with block updating. The study compared the four MCMC techniques for simplicity, ease of use, statistical efficiency in exploring the parameter space, and speed of implementation using 11 years of daily rainfall-runoff data. It was shown that the adaptive Metropolis algorithm is superior in many respects and can offer a relatively simple basis for assessing parameter uncertainty in hydrologic modeling studies.

For the Amchitka flow models, recharge and hydraulic conductivity are strongly correlated (Hassan et al., 2002). So, it is convenient to use the adaptive Metropolis algorithm (Marshall et al., 2004). For sake of completeness, more details of the traditional MetropolisHastings algorithm and the details of the adaptive Metropolis algorithm are provided here. These details are largely extracted from Marshall et al. (2004), but the discussion is adapted for the groundwater flow models at Amchitka.

\subsubsection{Metropolis-Hastings Algorithm}

The Metropolis-Hastings algorithm (Hastings, 1970) is a modification of the original Metropolis algorithm (Metropolis et al., 1953). The posterior distribution of the model parameters $\Theta=\left(\theta_{1}, \theta_{2}, \ldots, \theta_{N}\right)$ is sampled as follows:

1) Initialize $i=0$

2) Generate a proposed value $\Theta^{*}$ for $\Theta$ from a proposal density $q_{i}\left(\Theta^{*} \mid \Theta^{i}\right)$ depending on the current value $\Theta^{i}$ of $\Theta$. Then, the acceptance probability, $\alpha$, of the proposed value is computed as

$$
\alpha=\min \left\{1, \frac{P\left(\mathbf{D} \mid \Theta^{*}\right) P\left(\Theta^{*}\right) q\left(\Theta^{i} \mid \Theta^{*}\right)}{P\left(\mathbf{D} \mid \Theta^{i}\right) P\left(\Theta^{i}\right) q\left(\Theta^{*} \mid \Theta^{i}\right)}\right\}
$$

where $P(\mathbf{D} \mid \Theta)$ is the likelihood function of the observed data $\mathbf{D}$, and $P(\Theta)$ is the prior distribution of $\Theta$.

3) Generate a random number $u$ from a uniform distribution $U[0,1]$.

4) If $u<\alpha$, accept $\Theta^{i+1}=\Theta^{*}$, otherwise set $\Theta^{i+1}=\Theta^{i}$. 


\section{5) Repeat steps 2 through 4 .}

The above algorithm generally requires the specification of a multivariate proposal distribution for $\Theta$. Choosing the proposal to give a good acceptance rate (the rate of accepting the proposed value $\Theta^{*}$ ) is difficult when $\Theta$ is high dimensional (too many model parameters). In this case, the set of model parameters $\Theta$ is split into blocks $\Theta=\left(\Theta_{1}, \Theta_{2}, \ldots\right.$, $\Theta_{k}$ ) and then the Metropolis-Hastings algorithm is used to update each block $\Theta_{j}$ while keeping the other parameters (denoted as $\Theta_{\mathrm{j}}$ ) fixed. Sweeping through the blocks can then be performed either systematically or in some random order. The procedure for the case of systematic sweeping through the blocks is described in detail here.

Let $\Theta_{<j}=\left(\Theta_{1}, \Theta_{2}, \ldots, \Theta_{j-1}\right), \Theta_{>j}=\left(\Theta_{j+1}, \Theta_{j+2}, \ldots, \Theta_{k}\right)$, and write $\boldsymbol{\Theta}_{l_{j}}^{i}, \Theta_{<j}^{i}$, and $\Theta_{>j}^{i}$ for the values of $\boldsymbol{\Theta}_{\backslash j}, \Theta_{<j}$, and $\Theta_{>j}$, respectively, when $\Theta=\Theta^{i}$. In the block at a time Metropolis-Hastings algorithm, steps 2 through 5 above are replaced by the following:

For $j=1,2, \ldots, k$

1) Generate a proposed value $\Theta_{j}^{*}$ for $\Theta_{j}^{i+1}$ from a proposal density $q\left(\Theta_{j} \mid \Theta_{<j}^{i+1}, \Theta_{>j}^{i}\right)$.

2) Writing $\Theta^{*}=\left(\Theta_{<j}^{i+1}, \Theta_{j}^{*}, \Theta_{>j}^{i}\right)$ and $\Theta^{c}=\left(\Theta_{<j}^{i+1}, \Theta_{j}^{i}, \Theta_{>j}^{i}\right)$, the acceptance probability, $\alpha$, can be computed as

$$
\alpha=\min \left\{1, \frac{P\left(\mathbf{D} \mid \Theta^{*}\right) P\left(\Theta^{*}\right) q\left(\Theta_{j}^{i} \mid \Theta_{<j}^{i+1}, \Theta_{>j}^{i}\right)}{P\left(\mathbf{D} \mid \Theta^{c}\right) P\left(\Theta^{c}\right) q\left(\Theta_{j}^{*} \mid \Theta_{<j}^{i+1}, \Theta_{>j}^{i}\right)}\right\}
$$

3) Generate a random number $u$ from a uniform distribution $U[0,1]$.

4) If $u<\alpha$, accept $\Theta_{j}^{i+1}=\Theta_{j}^{*}$, otherwise set $\Theta_{j}^{i+1}=\Theta_{j}^{i}$.

5) Perform an increment $j$ (i.e., $j=j+1$ ) until $j=k$

6) Repeat steps 2 through 6 .

As stated earlier, the difficulty in this approach lies in the choice of appropriate proposal density especially when the number of parameters is large. It should be noted that if two or more of the parameters are highly interdependent, they should be updated together as a block, otherwise the convergence of the process to its stationary distribution may take an excessively large number of iterations. This, however, requires the specification of the joint proposal distribution for parameters in the block, which is usually difficult. In the adaptive Metropolis algorithm discussed next, all the parameters are updated simultaneously.

\subsubsection{Adaptive Metropolis Algorithm}

The adaptive Metropolis (AM) algorithm presented by Haario et al. (2001) is a variation of the conventional Metropolis algorithm. The AM algorithm relies on a proposal distribution that benefits from the generated samples (from the posterior distribution) at each iteration. The proposal density uses the estimated posterior covariance matrix of the parameters, which is computed at each iteration based on past iterations. In this manner, the proposal density is updated using the current knowledge about the posterior distribution 
(Marshall et al., 2004). At step $i$, a multivariate normal proposal with mean given by the current value and covariance matrix $\operatorname{Cov}_{i}$ is used. This covariance assumes a fixed value for the first $i_{0}$ iterations and is subsequently updated by

$$
\operatorname{Cov}_{i}=\left\{\begin{array}{lr}
\operatorname{Cov}_{0} & i \leq i_{0} \\
S_{d} \operatorname{Cov}\left(\Theta^{0}, \Theta^{1}, \ldots, \Theta^{i-1}\right) & i>i_{0}
\end{array}\right.
$$

where $S_{d}$ is a scaling parameter that depends on the dimensionality $d$ of $\Theta$ and is used to ensure a reasonable acceptance rate of the proposed values. As a rule of thumb, Haario et al. (2001) suggest choosing $S_{d}$ for a model with $d$ parameters as $(2.4)^{2} / d$. The covariance at iteration $i+1$ can be easily calculated from the recursion formula (Marshall et al., 2004)

$$
\operatorname{Cov}_{i+1}=\frac{i-1}{i} \operatorname{Cov}_{i}+\frac{S_{d}}{i}\left[i \bar{\Theta}^{i-1}\left(\bar{\Theta}^{i-1}\right)^{T}-(i-1) \bar{\Theta}^{i}\left(\bar{\Theta}^{i}\right)^{T}+\Theta^{i}\left(\Theta^{i}\right)^{T}\right]
$$

where $\bar{\Theta}^{i}$ is the mean of parameter values $\Theta^{0}, \Theta^{1}, \ldots$, and $\Theta^{i}$ generated in iterations $0,1, \ldots$, and $i$. Using Equation (3.8) to compute the proposal covariances requires an arbitrary initial covariance, $\operatorname{Cov}_{0}$. To automate the process, Marshall et al. (2004) suggest setting the initial covariance as the covariance of parameters under their prior distributions.

Based on the above discussion, the steps involved in implementing the AM algorithm are as follows:

1) Initialize $i=0$.

2) Select $\operatorname{Cov}_{i}$ for current iteration, $i$, using Equation (3.7). Generate a proposed value $\Theta^{*}$ for $\Theta$ from a proposal density that can be taken as multinormal $\sim \mathrm{MN}\left(\Theta^{i}, \operatorname{Cov}_{i}\right)$.

Compute the acceptance probability, $\alpha$, of the proposed value as

$$
\alpha=\min \left\{1, \frac{P\left(\mathbf{D} \mid \Theta^{*}\right) P\left(\Theta^{*}\right)}{P\left(\mathbf{D} \mid \Theta^{i}\right) P\left(\Theta^{i}\right)}\right\}
$$

where $P(\mathbf{D} \mid \Theta)$ is the likelihood function of the observed data $\mathbf{D}$, and $P(\Theta)$ is the prior distribution of $\Theta$.

3) Generate a random number $u$ from a uniform distribution $U[0,1]$.

4) If $u<\alpha$, accept $\Theta^{i+1}=\Theta^{*}$, otherwise set $\Theta^{i+1}=\Theta^{i}$.

5) Repeat steps 2 through 4.

If constraints exist on the parameters (minimum or maximum plausible values), the acceptance probability should be set to zero for proposed values that are outside these constraints. The major advantage of the AM algorithm is that the entire parameter set is updated simultaneously, which reduces computational time and complexity. This is particularly useful when the parameters are highly correlated, as is the case for Amchitka models. 


\section{RECALIBRATION, VERIFICATION AND UNCERTAINTY REDUCTION USING MCMC}

The AM algorithm is applied to the groundwater flow models of Amchitka for the purpose of recalibrating the models with the newly collected data of CRESP and reducing parametric uncertainty. The conceptual and numerical groundwater flow models for the three tests are briefly summarized in Section 2. These same models, along with old and new data, are used here to develop posterior distributions for the important parameters governing the flow regime underneath the island: recharge, conductivity, and the recharge-conductivity ratio.

\subsection{Data Description}

Detailed descriptions of data pertaining to the Amchitka models can be found in Hassan et al. (2002). Described here are 1) the original data that are either used in the MCMC conditioning (e.g., salinity and head data) or impacted by the information gathered by CRESP (e.g., effective porosity, bathymetric profiles), and 2) the new CRESP data that are used in the MCMC conditioning (i.e., MT data). These data sets are discussed and will form the basis for conditioning and constraining the model parameters through the use of the Bayesian MCMC method as well as subsequent flow and transport modeling.

\subsubsection{Head Data used in the 2002 Model}

Water levels measured during hydraulic testing are used to represent hydraulic heads at the depths of the packed intervals. Unfortunately, the effects of packer bypass and the incomplete recovery of water levels after swabbing in many tests reduced the number of reliable data available for characterizing the spatial distribution of hydraulic head; many of the values reported are noted as "estimated" or "assumed" based on measurements made under similar conditions in other intervals or other boreholes. Of the data obtained from packer tests, only water levels that clearly indicate static conditions, either prior to an injection test or upon full recovery after a swabbing test, are used in the flow model calibrations. These water levels are assumed to represent hydraulic head at the depth of their measurement. The influence of groundwater salinity and temperature on the measurements is unknown because these parameters were not consistently reported with the water level data, thus the measured head values could not be corrected to represent equivalent freshwater heads. This uncertainty increases with increased depth as the salinity and the heat effects on water density increase with depth.

Measurements of static water levels in various shallow observation wells are used to augment the packer test data, which were generally collected at depths greater than $500 \mathrm{~m}$. Construction details and exact locations of many of the shallow wells are not available, but water levels consistently indicate that the water table lies within several meters of ground surface and that heads decline with increasing depth. The hydraulic head data used for calibration of the three models is given in Table 4.1 and shown in Figure 4.1. Additional head data for the island can be found tabulated and evaluated in Hassan et al. (2002). 
Table 4.1. Hydraulic head calibration targets used in Hassan et al. (2002).

\begin{tabular}{|c|c|c|c|c|c|c|}
\hline \multirow[b]{2}{*}{$\begin{array}{l}\text { Well } \\
\text { name }\end{array}$} & \multicolumn{3}{|c|}{ Depth (m) } & \multicolumn{3}{|c|}{ Elevation (m) } \\
\hline & $\begin{array}{l}\text { Interval } \\
\text { top }\end{array}$ & $\begin{array}{l}\text { Interval } \\
\text { bottom }\end{array}$ & $\begin{array}{c}\text { Static water } \\
\text { level }\end{array}$ & $\begin{array}{l}\text { Interval } \\
\text { top }\end{array}$ & $\begin{array}{l}\text { Interval } \\
\text { bottom }\end{array}$ & $\begin{array}{c}\text { Static water } \\
\text { level }\end{array}$ \\
\hline \multicolumn{7}{|l|}{ Milrow Wells } \\
\hline UAe-2 & 379.8 & 440.1 & 14.2 & -340.33 & -400.63 & 25.27 \\
\hline $\mathrm{UAe}-2$ & 719.3 & 779.6 & 19.5 & -679.83 & -740.13 & 19.97 \\
\hline UAe-2 & 933.9 & 994.3 & 27 & -894.43 & -954.83 & 12.47 \\
\hline UAe-2 & $1,057.6$ & $1,127.8$ & 19.7 & $-1,018.1$ & $-1,088.3$ & 19.77 \\
\hline UAe-2 & $1,057.6$ & $1,127.8$ & 25 & $-1,018.1$ & $-1,088.3$ & 14.47 \\
\hline \multicolumn{7}{|c|}{ Long Shot Wells } \\
\hline EH-5 & 663.2 & 724.2 & 14.2 & -621.1 & -682.14 & 27.9 \\
\hline EH-5 & 723.6 & 784.6 & 16 & -681.5 & -742.5 & 25.7 \\
\hline $\mathrm{EH}-5$ & 723.6 & 784.6 & 16.9 & & & \\
\hline W-1 & & 1.1 & 0 & & 40.94 & 42.05 \\
\hline WL-1 & & 2.7 & 0.8 & & 39.41 & 41.26 \\
\hline WL-2 & & 3.4 & 1 & & 38.7 & 41.1 \\
\hline Well-1 & & 37.8 & 1.7 & & 4.27 & 40.36 \\
\hline Well-3 & & 37.5 & 2.5 & & 4.57 & 39.55 \\
\hline Well-8 & & 37.5 & 0.8 & & 4.57 & 41.25 \\
\hline Well-9 & & 100.9 & 4.7 & & -58.83 & 37.4 \\
\hline USGS & & 213.3 & 0.6 & & -171.3 & 41.51 \\
\hline \multicolumn{7}{|l|}{ Cannikin Wells } \\
\hline White Alice $^{\mathrm{a}}$ & & 96.3 & 1.82 & & -7.9 & 86.58 \\
\hline UA-1 & $1,664.5$ & $1,968.4$ & 37.8 & $-1,601.2$ & $-1,905.1$ & 25.52 \\
\hline UA-1 & $1,693.5$ & $1,753.8$ & 35 & $-1,630.2$ & $-1,690.5$ & 28.32 \\
\hline UA-1 & $1,806.3$ & $1,859.9$ & 36.4 & $-1,743$ & $-1,796.6$ & 26.92 \\
\hline UA-1 & 1,813 & $1,873.3$ & 38.7 & $-1,749.7$ & $-1,810$ & 24.62 \\
\hline UA-1 & $1,834.9$ & $1,895.3$ & 37.7 & $-1,771.6$ & $-1,832$ & 25.62 \\
\hline UA-1 & $1,896.7$ & $1,968.4$ & 36.4 & $-1,833.4$ & $-1,905.1$ & 26.92 \\
\hline UA-1-HTH-1 ${ }^{\text {b }}$ & 80.5 & 125 & 3.9 & -30.37 & -74.87 & 46.23 \\
\hline UA-1-HTH-1 & 128.3 & 179.5 & 4 & -78.17 & -129.37 & 46.13 \\
\hline UA-1-HTH-1 & 183.5 & 234.7 & 4 & -133.37 & -184.57 & 46.13 \\
\hline UA-1-HTH-1 & 227.4 & 278.6 & 5.7 & -177.27 & -228.47 & 44.4 \\
\hline UAe-1 & 487.7 & 518.2 & 34.4 & -424.82 & -455.32 & 28.48 \\
\hline UAe-1 & 542.6 & 563.9 & 34.4 & -479.72 & -501.02 & 28.48 \\
\hline UAe-1 & 759 & 786.4 & 34.4 & -696.12 & -723.52 & 28.48 \\
\hline UAe-1 & 951 & 969.3 & 34.2 & -888.12 & -906.42 & 28.68 \\
\hline UAe-1 & $1,531.3$ & $1,580.1$ & 33.1 & $-1,468.4$ & $-1,517.2$ & 29.78 \\
\hline UAe-1 & $1,645.9$ & $1,724.6$ & 35.1 & $-1,583$ & $-1,661.7$ & 27.78 \\
\hline UAe-1 & $1,648.4$ & $2,133.6$ & 33.9 & $-1,585.5$ & $-2,070.7$ & 28.98 \\
\hline UAe-1 & 1,724 & $1,784.3$ & 37.7 & $-1,661.1$ & $-1,721.4$ & 25.18 \\
\hline
\end{tabular}

${ }^{\mathrm{a}}$ The White Alice borehole is located northwest of UA-1 and west of HTH-1. The shallow depth to water observed in White Alice was projected to the Cannikin site at UA-1.

${ }^{\mathrm{b}}$ The UA-1-HTH-1 borehole is located to the northwest of the model line running from UA-1 to the Bering Sea. The land surface elevation is higher at HTH-1 than where it projects to the section; therefore, the calibration heads at the model were adjusted for the elevation difference of $18.38 \mathrm{~m}$. 

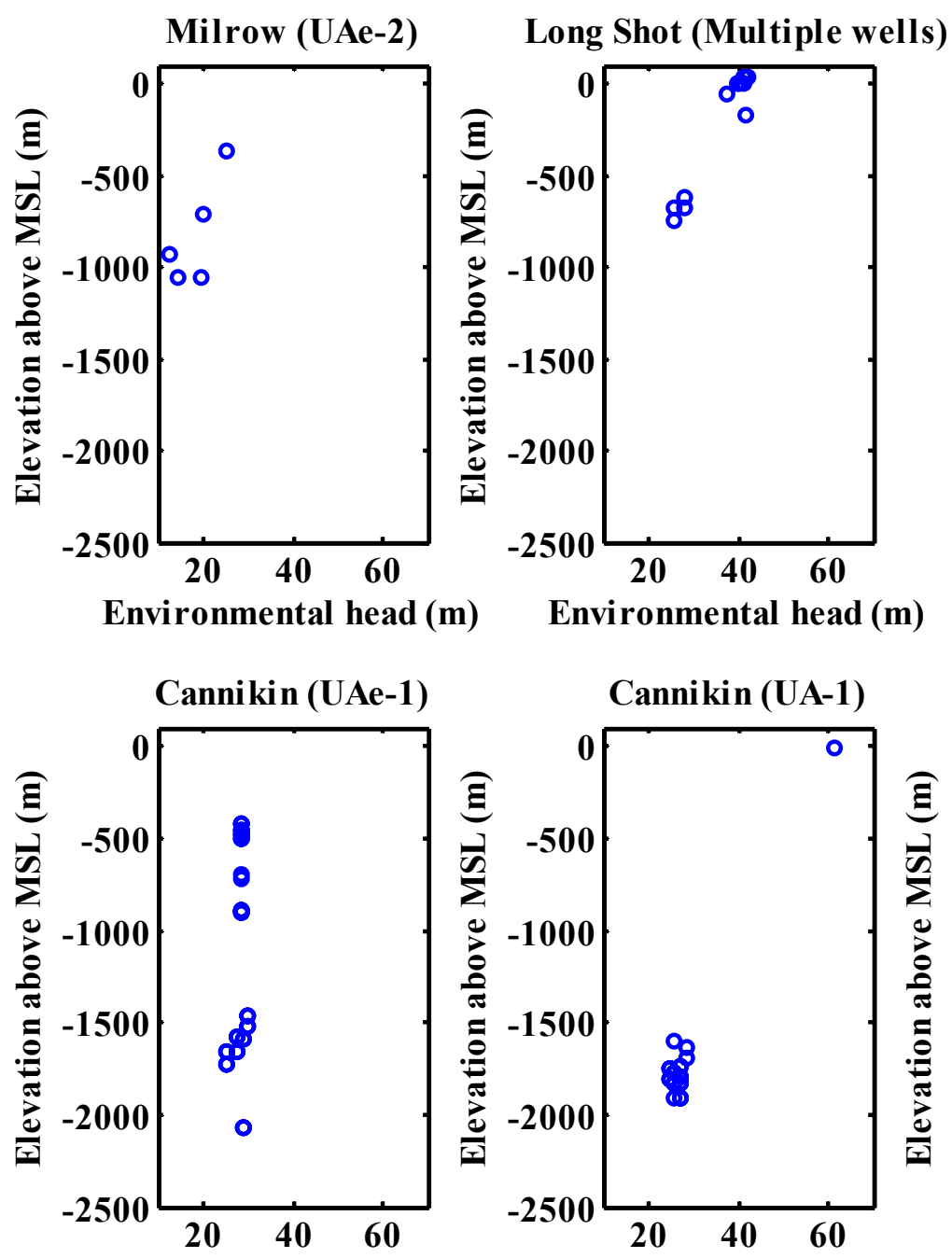

Environmental head (m)
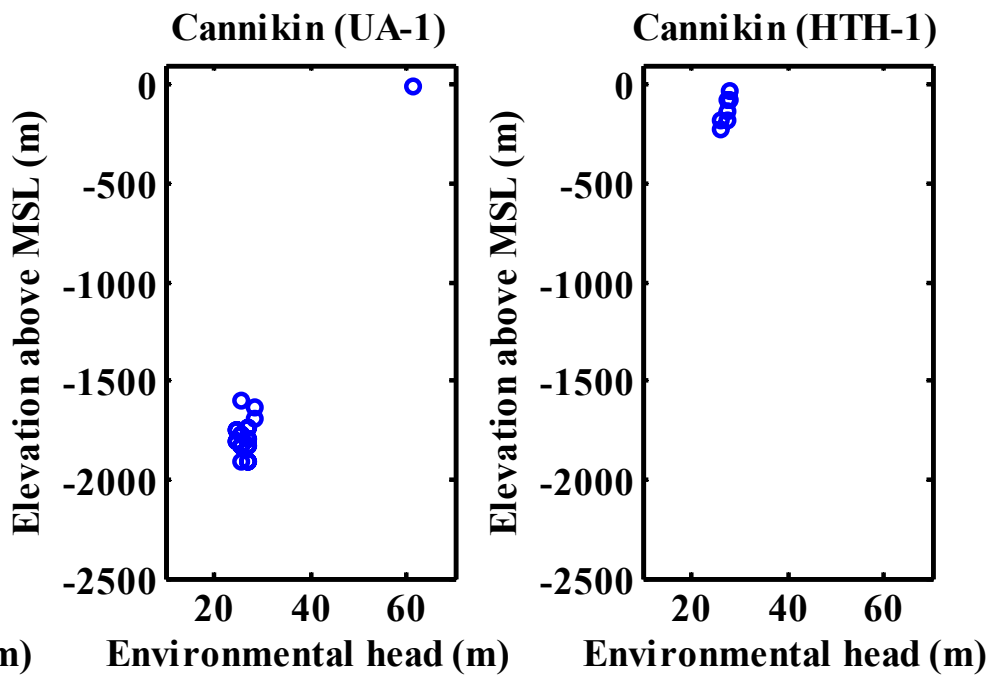

Figure 4.1. Head data used as calibration targets in the 2002 model of Hassan et al. These data sets are also used here in the MCMC conditioning along with the MT-converted concentration data and the salinity data.

\subsubsection{Salinity Data used in the 2002 Model}

Chemical data, sample collection and analytical procedures for water samples from the island are reported by Beetem et al. (1971). Groundwater samples from wells at Long Shot were analyzed and reported by the U.S. Army Corps of Engineers and U.S. Geological Survey (USGS) (1965), though many of their analyses were incomplete in that they did not include all major anions and cations.

The dissolved solids content of seawater collected off Amchitka is reported as $34,700 \mathrm{mg} / \mathrm{L}$ for the Bering Sea and 34,800 $\mathrm{mg} / \mathrm{L}$ for the Pacific Ocean (Beetem et al., 1971). Samples of surface water were collected from lakes and streams on the island and 
have a mean total dissolved solids (TDS) content of $145 \mathrm{mg} / \mathrm{L}$ and $137 \mathrm{mg} / \mathrm{L}$, respectively (from residue on evaporation, Beetem et al., 1971). Water from springs is similar, with a TDS of $143 \mathrm{mg} / \mathrm{L}$. These relatively high salinities for surface water reflect the influence of the near-coast environment and salt spray.

Groundwater samples from boreholes on Amchitka have primarily been collected by swabbing discrete intervals, though a few pumped samples from large intervals have also been collected. Collection of samples representative of formation water can be difficult due to the need to purge drilling fluids. High $\mathrm{pH}$ values are reported for some of the groundwater samples, indicating they were affected by contact with cement during grouting operations.

The groundwater-freshwater lens and underlying saltwater can be identified from samples collected by swabbing packer-isolated zones in well UAe-2. These zones are typically about $60 \mathrm{~m}$ in length and thus the samples represent composites of borehole fluid through that length. The chemical analysis of these samples (Beetem et al., 1971) clearly defines the increase in salinity with depth to near-seawater concentrations. At Long Shot, electrical conductivity measurements led workers to identify "saltwater encroachment" in deeper groundwater samples, but salinities close to seawater were not found in chemical analysis. Groundwater from 670 to $793 \mathrm{~m}$ below land surface at Long Shot contained 350 to 415 ppm chloride and TDS contents of 1,460 to 1,650 mg/L (U.S. Army Corps of Engineers and USGS, 1965). Water samples from borehole UAe-1 at the Cannikin site show little relationship between salinity and depth.

Using chloride as a conservative-ion indicator of salinity, the transition zone midpoint can be defined as the depth where the groundwater has a chloride content midway between the surface water value and the seawater value (i.e., $9,025 \mathrm{mg} / \mathrm{L}$, halfway between about 50 and $18,100 \mathrm{mg} / \mathrm{L} \mathrm{Cl}$ ). At Milrow, that occurs at an elevation of about $850 \mathrm{~m}$ below sea level, and at the other two sites, that salinity is not achieved in water from the sampled intervals. The chloride values, and sample-to-seawater chloride ratios used for model calibration, are presented in Table 4.2 and shown in Figure 4.2. Complete chemical analyses and additional discussion are in Hassan et al. (2002).

\subsubsection{Magnetotelluric Data Collected by CRESP in 2004}

During the 2004 CRESP Amchitka Island Expedition, MT data were collected on transects across the three test sites, covering the width of the island at Cannikin, about 75 percent of the width at Long Shot, and about half of the width at Milrow. The MT data were collected through 12 stations across the island at Cannikin, 10 stations at Long Shot and 6 stations at Milrow. After processing and inverting the MT data (Unsworth et al., 2005), twodimensional models of subsurface electrical resistivity were derived. These showed a pattern of increasing, decreasing and increasing resistivity was observed at each test site on Amchitka Island. The inflection point of the resistivity profile, where resistivity begins to decrease, is interpreted by Unsworth et al. (2005) as the top of the TZ where salinity increases. The deeper inflection point (increase in resistivity after its decrease with depth) is interpreted as the base of the transition zone, where salinity remains constant and porosity decreases (Unsworth et al., 2005). 
Table 4.2. Groundwater chemistry calibration targets used in Hassan et al. (2002).

\begin{tabular}{|c|c|c|c|c|c|c|}
\hline \multirow[b]{2}{*}{ Well Name } & \multicolumn{2}{|c|}{ Depth (m) } & \multirow{2}{*}{$\begin{array}{l}\text { Depth }(\mathrm{m}) \\
\text { mid-point }\end{array}$} & \multirow{2}{*}{$\begin{array}{c}\text { Elevation }(\mathrm{m}) \\
\text { mid-point }\end{array}$} & \multirow[b]{2}{*}{$\mathrm{Cl}, \mathrm{mg} / \mathrm{L}$} & \multirow[b]{2}{*}{$\mathrm{Cl} / \mathrm{Cl}_{\text {sea }}$} \\
\hline & Interval top & Interval bottom & & & & \\
\hline \multicolumn{7}{|l|}{ Milrow } \\
\hline Surface water & 0 & 0 & 0 & 39.47 & 48 & 0.003 \\
\hline \multirow[t]{9}{*}{ UAe-2 } & 379.8 & 440.1 & 409.95 & -370.48 & 3,000 & 0.166 \\
\hline & 719.3 & 779.7 & 746.5 & -707.03 & 8,100 & 0.448 \\
\hline & 822.4 & 882.7 & 852.55 & -813.08 & 11,900 & 0.657 \\
\hline & 873.6 & 933.9 & 903.75 & -864.28 & 12,000 & 0.663 \\
\hline & 933.9 & 994.3 & 964.1 & -924.63 & 11,900 & 0.657 \\
\hline & $1,057.6$ & $1,127.8$ & $1,092.7$ & $-1,053.23$ & 11,900 & 0.657 \\
\hline & $1,169.8$ & $1,230.2$ & 1,200 & $-1,160.53$ & 15,000 & 0.829 \\
\hline & $1,466.7$ & 1,527 & $1,496.85$ & $-1,457.38$ & 16,000 & 0.884 \\
\hline & $1,530.1$ & $1,590.4$ & $1,560.25$ & $-1,520.78$ & 16,000 & 0.884 \\
\hline \multicolumn{7}{|l|}{ Long Shot } \\
\hline \multirow[t]{7}{*}{ EH-5 } & 390 & 451 & 420.5 & -378.44 & 65 & 0.004 \\
\hline & 390 & 451 & 420.5 & -378.44 & 71 & 0.004 \\
\hline & 602 & 663 & 632.5 & -590.44 & 337 & 0.019 \\
\hline & 662.9 & 723.9 & 693.4 & -651.34 & 351 & 0.019 \\
\hline & 663 & 724 & 693.5 & -651.44 & 334 & 0.018 \\
\hline & 723.9 & 784.6 & 754.3 & -712.24 & 417 & 0.023 \\
\hline & 724 & 785 & 754.5 & -712.44 & 405 & 0.022 \\
\hline \multirow[t]{2}{*}{ EE hole } & 45.7 & 50.3 & 48 & -5.94 & 76 & 0.004 \\
\hline & 122.5 & 126.5 & 125 & -82.94 & 500 & 0.028 \\
\hline \multicolumn{7}{|l|}{ Cannikin } \\
\hline \multirow[t]{11}{*}{ UAe-1 } & 951.2 & 969.5 & 960.35 & -896.95 & 690 & 0.038 \\
\hline & $1,356.7$ & $1,387.2$ & $1,371.95$ & $-1,308.55$ & 2,000 & 0.110 \\
\hline & $1,643.9$ & $1,655.5$ & $1,649.7$ & $-1,586.3$ & 1,850 & 0.102 \\
\hline & $1,646.3$ & 1,725 & $1,685.65$ & $-1,622.25$ & 1,860 & 0.103 \\
\hline & $1,646.3$ & 1,725 & $1,685.65$ & $-1,622.25$ & 432 & 0.024 \\
\hline & $1,724.4$ & $1,784.8$ & $1,754.6$ & $-1,691.2$ & 2,060 & 0.114 \\
\hline & 1,786 & $1,826.2$ & $1,806.1$ & $-1,742.7$ & 2,040 & 0.113 \\
\hline & $1,802.4$ & $1,862.2$ & $1,832.3$ & $-1,768.9$ & 1,190 & 0.066 \\
\hline & $1,802.4$ & $1,862.2$ & $1,832.3$ & $-1,768.9$ & 1,260 & 0.070 \\
\hline & $1,850.6$ & 1,911 & $1,880.8$ & $-1,817.4$ & 1,740 & 0.096 \\
\hline & $1,850.6$ & 1,911 & $1,880.8$ & $-1,817.4$ & 1,770 & 0.098 \\
\hline
\end{tabular}

${ }^{\mathrm{a}}$ Mean value of 10 samples of lakes and streams near Milrow. 


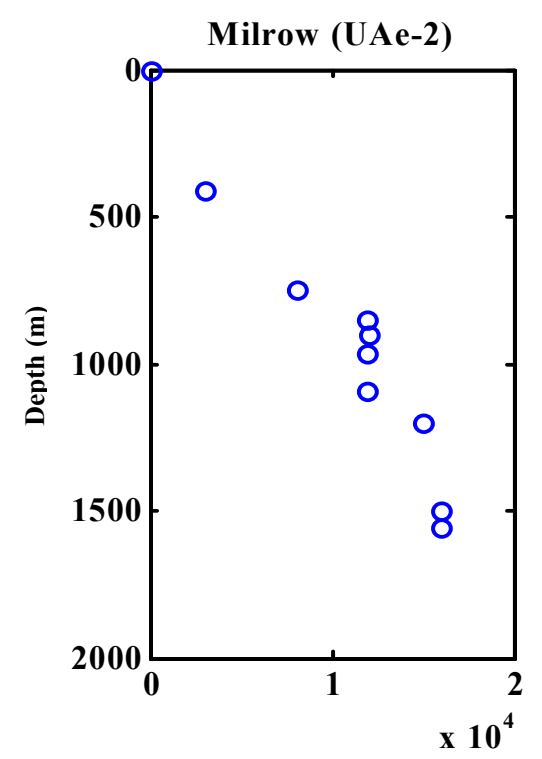

Cl Conc. $(\mathrm{mg} / \mathrm{L})$

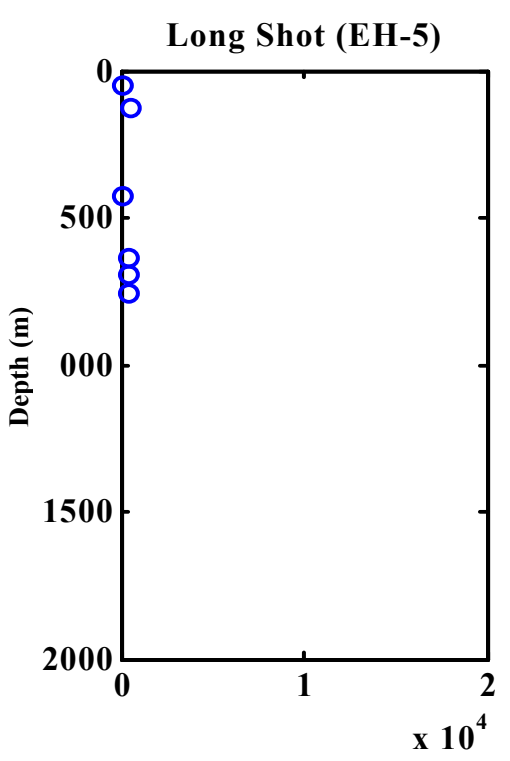

Cl Conc. $(\mathrm{mg} / \mathrm{L})$

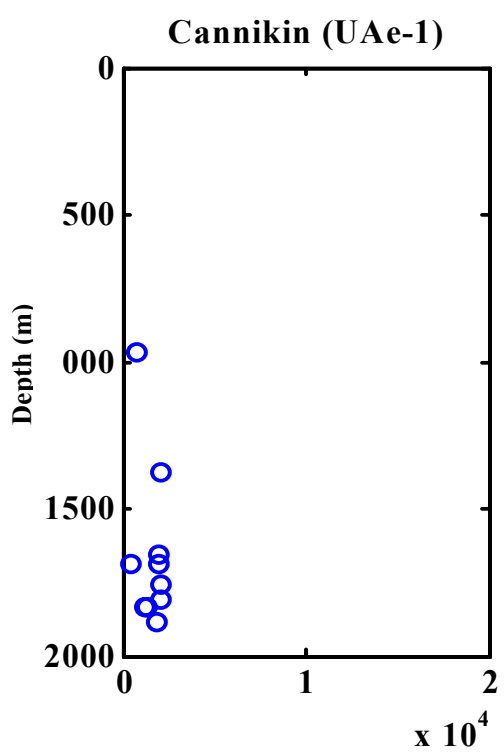

Cl Conc. $(\mathrm{mg} / \mathrm{L})$

Figure 4.2. Salinity data used as calibration targets in the 2002 model of Hassan et al. These data sets are also used here in the MCMC conditioning along with the MT-converted concentration data and the head data.

The depths interpreted from the resistivity models of Unsworth et al. (2005) indicate that the top of the TZ at Milrow is at about $900 \mathrm{~m}$ below ground surface and the bottom is at $1,700 \mathrm{~m}$. Uncertainties were estimated in some parameters (Unsworth et al., 2005) which resulted in a possible range for the $\mathrm{TZ}$ top location of 800 to $1,110 \mathrm{~m}$ and a range for the $\mathrm{TZ}$ bottom location of 1,500 to $2,100 \mathrm{~m}$. For Long Shot, the top of the TZ is estimated to be 600 $\mathrm{m}$ deep with a possible range of 500 to $1,000 \mathrm{~m}$, and the bottom is estimated to be $1,700 \mathrm{~m}$ deep with a range of 1,500 to $2,000 \mathrm{~m}$. At Cannikin, the top of the TZ is estimated at $900 \mathrm{~m}$ below ground surface with uncertainty range of 800 to $1,000 \mathrm{~m}$ and the bottom is at 2,500 m with a possible range of 2,000 to $2,700 \mathrm{~m}$ (Figure 4.3). Though these results do not give the variation of salinity with depth, they indicate where the top and bottom of the TZ might be located. As is explained later, the use of this data set for conditioning requires some assumptions and abstraction. 


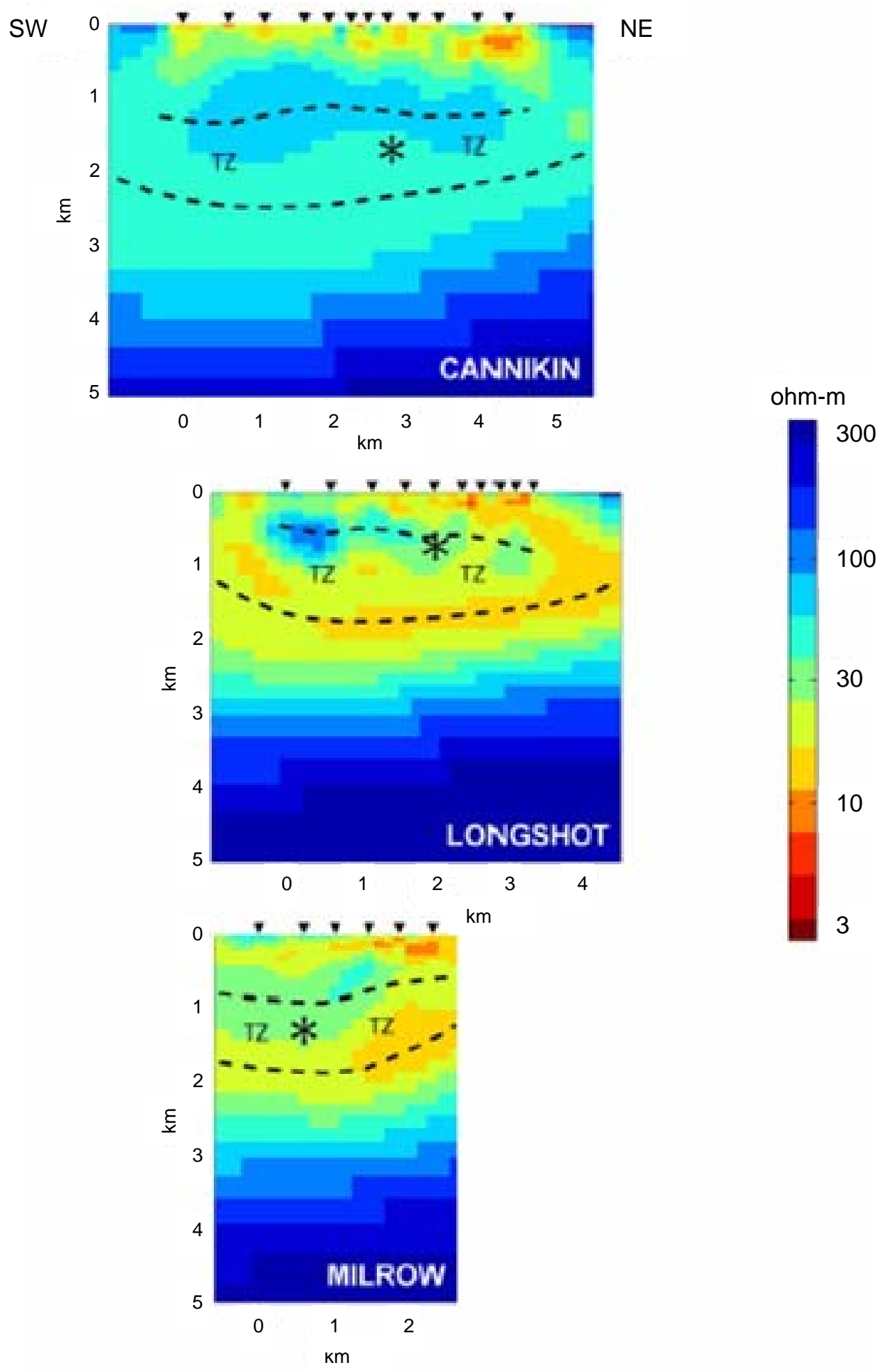

Figure 4.3. Resistivity profiles as obtained from the inversion models used in conjunction with the MT data. The dotted lines show the inferred locations of the top and bottom of the TZ where resistivity begins to decrease and increase, respectively. The asterisks indicate the cavity location at the three sites (adopted from Unsworth et al., 2005). 


\subsubsection{Effective Porosity}

The 2002 models were based on a conceptual model of fracture flow. A dual-porosity system was used since relatively high interstitial porosity values were measured in cores (discussed below), and the primary flow system is assumed to be through fractured rock.

Measurements of total porosity of rocks undisturbed by nuclear test effects were made on 197 core samples obtained from four boreholes at Amchitka (Lee, 1969a, b, c, d). An arithmetic mean of 0.14 and a standard deviation of 0.08 was calculated for samples from all depths, and a mean of 0.12 and a standard deviation of 0.06 was calculated for rocks from the deeper Kirilof Point and Older Breccias (Figure 4.4). These measurements represent matrix porosity of blocks of basalt and breccia that occupy the volume between connected fractures, and are likely to overestimate the effective flow porosity in the fracture zones themselves.

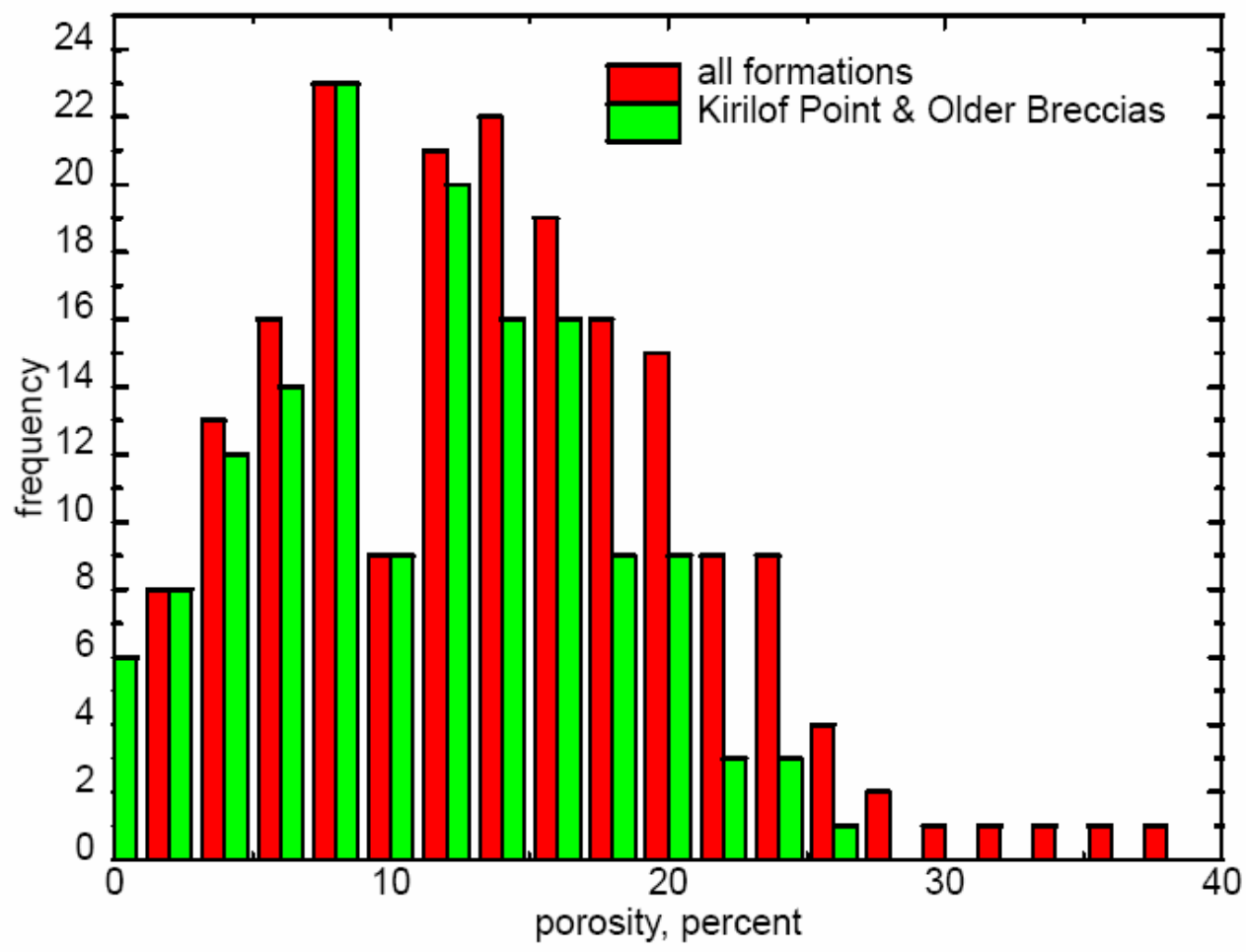

Figure 4.4. Variation of total porosity determined from laboratory tests on core samples.

The U.S. Army Corps of Engineers and USGS (1965) estimated an effective porosity of $1.0 \times 10^{-2}$ from hydraulic testing in EH-1, and $1.0 \times 10^{-3}$ from hydraulic testing in EH-5 (both at the Long Shot site). Nork et al. (1965) assumed a value of $1.0 \times 10^{-3}$ for flow in joints in andesite and a value of $1.0 \times 10^{-2}$ for combined fracture and interstitial flow at the Long Shot site. Essington et al. (1970) used effective porosities of $1.5 \times 10^{-3}$ to $2.5 \times 10^{-3}$. By analyzing the response in well UAe-1 to tidal and barometric effects, Fenske (1972) estimated a fracture system porosity of $1 \times 10^{-3}$. Dudley et al. (1977) used estimates of fracture spacing and $K$ data and an empirical relationship developed by Snow (1968) to estimate fracture porosities between $1.9 \times 10^{-5}$ and $6.3 \times 10^{-4}$. Though the hydraulic response 
estimates had the fewest uncertainties, a lower value of $5.0 \times 10^{-4}$ was used in the 2002 model to represent the fracture porosity of undisturbed rock. This was a conservative selection given the inverse relationship of porosity and groundwater velocity, and used as a mean value around which distributions of porosity were developed to address uncertainty.

Though the porosity distributions (input to the random field generator [RFG]) were similar for the three models, there were slight differences driven by the different number of realizations and different speed of the RFG. The distribution used for each of the 2002 models is given in Table 4.3.

Table 4.3. Porosity distributions used to generate realizations for each model reported in Hassan et al. (2002).

\begin{tabular}{|l|l|c|c|c|c|c|c|c|c|}
\hline \multirow{3}{*}{ Model } & \multicolumn{3}{|c|}{ Input to the RFG } & \multicolumn{6}{c|}{ Output of the RFG } \\
\cline { 2 - 9 } & Distribution & $\log _{10}(n)$ & $\log _{10}(n)$ & \multicolumn{4}{|c|}{$n$} \\
\cline { 3 - 10 } & mean & $\sigma$ & mean & $\sigma$ & minimum & mean & maximum & $\sigma$ \\
\hline Milrow & lognormal & -3.6 & 0.48 & -3.595 & 0.466 & $1.057 \times 10^{-5}$ & $4.4 \times 10^{-4}$ & $6.2 \times 10^{-3}$ & $5.92 \times 10^{-4}$ \\
\hline Long Shot & lognormal & -3.6 & 0.48 & -3.625 & 0.471 & $1.024 \times 10^{-5}$ & $4.11 \times 10^{-4}$ & $5.2 \times 10^{-3}$ & $5.19 \times 10^{-4}$ \\
\hline Cannikin & lognormal & -3.6 & 0.48 & -3.606 & 0.474 & $1.0 \times 10^{-5}$ & $4.31 \times 10^{-4}$ & $5.2 \times 10^{-3}$ & $5.4 \times 10^{-4}$ \\
\hline
\end{tabular}

By combining the MT resistivity profiles with salinity data from wells, Unsworth et al. (2005) modeled porosity profiles for the Amchitka subsurface. There are a number of assumptions and empirical relationships involved in the analysis, and a thorough description of these is provided by Unsworth et al. (2005). They note that their estimates are of effective porosity, i.e, interconnected pore space. The MT data suggest that porosity decreases with depth from a value of approximately $3 \times 10^{-1}$ near the surface at all three tests to $2 \times 10^{-2}$ at a depth of 3,000 $\mathrm{m}$ at Long Shot and Milrow, and to $3 \times 10^{-2}$ at a depth of 3,000 $\mathrm{m}$ at Cannikin.

The MT effective porosity estimates are significantly higher than those used in the original groundwater models. However, low porosity values were deliberately selected for the original modeling in an effort to be conservative (producing faster velocities), and some evidence pointed to higher values, such as the hydraulic response estimates discussed previously. A parametric uncertainty analysis identified porosity as significant in terms of impact on the contaminant transport. Despite the abundant core data, effective porosity is poorly known for fracture-flow systems. Thus, a decision was made in the original modeling to conservatively skew the distribution to porosity values that promoted contaminant transport. The MT porosity analysis suggests that the modeling will be more realistic with higher porosity values.

The recent effective porosity information was applied in the groundwater model following the processes described below. Unsworth et al. (2005) present three porosity profiles for each site, developed using three different cementation factors (Figure 4.5). The cementation factor is a key control parameter in Archie's Law, an empirical relationship between resistivity and porosity that was used to develop the porosity profiles. Given that the cementation factor is unknown, but critical to the analysis, Unsworth et al. (2005) considered a range of values and this produced several porosity profiles. The lowest and highest profiles 
were used to bound the porosity profiles for each site. For each model realization, a porosity profile is randomly generated that fits between the two bounds. The porosity is uniform in the model domain in the horizontal direction, but varies vertically according to the MT-derived profile. The original model applied a random, but uniform, porosity in all directions.

The porosity of the nuclear cavity and chimney also needed to be considered. The collapse of rock overlying the nuclear cavity creates a rubble-filled "chimney" of rock. Though the portions occupied by nuclear melt glass and pulverized rock flour may have very low porosity and permeability, they are a small part of the cavity-chimney volume, and overall the collapse and seismic disruption are generally observed to increase porosity. This conceptual model is implemented by assigning the cavity-chimney complex a higher porosity than the surrounding rock.

In the 2002 groundwater models, the porosity of the nuclear chimneys was $7 \times 10^{-2}$, based upon estimates provided by Fenske (1972) for Milrow and by Claassen (1978) for Cannikin. These studies used observed groundwater infill behavior to estimate chimney parameters, but significantly, this also required assumptions regarding the hydraulic properties of the surrounding rock. These uncertainties were easily tolerated in the previous model because the estimate of chimney porosity was orders of magnitude larger than the values assigned to the rest of the subsurface. The higher porosities indicated by the MT analysis require a more critical evaluation of the estimated chimney porosity.

Fenske (1972) calculated the distribution of rubble porosity in the Milrow chimney to be zero at the bottom (in the puddle glass) to $1.4 \times 10^{-1}$ at the top. Claassen (1978) estimated the distribution in the Cannikin chimney to be $1 \times 10^{-1}$ near the bottom and $4 \times 10^{-2}$ near the top. Borg et al. (1976) present bulking porosities for a range of competent rock types (e.g., granite, basalt, indurated tuffs) that range from $1.8 \times 10^{-1}$ to 3.5 and $10^{-1}$. While evaluating the French nuclear tests at volcanic islands in the South Pacific, the International Atomic Energy Agency (IAEA) (1998) estimated that a cavity-chimney created by a nuclear explosion has an overall porosity of about $3 \times 10^{-1}$. Recent workers examining contaminant transport at the Nevada Test Site used cavity-chimney porosities of $1 \times 10^{-1}$ for a cavitychimney in volcanic terrain (Pawloski et al., 2001) and $3.8 \times 10^{-1}$ for a cavity-chimney in alluvium (Tompson et al., 1999).

To be consistent with the conceptual model, the chimney porosity for a given realization should be higher than the porosity of the surrounding rock. However, given the large porosity values estimated by the MT analysis for the shallow subsurface, it may be unrealistic to assign larger chimney porosity values. An analogy can be found in Tompson et al. (1999), where the $3.8 \times 10^{-1}$ value used for the cavity-chimney is the same as that assigned to the surrounding alluvium.

Given the considerations described above, chimney porosity was assigned the highest value indicated by the MT data for Milrow, which was about 0.4 . This chimney porosity is applied for every realization for all three sites, and is assigned uniformly from the cavity location to the land surface. The porosity profiles of Unsworth et al. (2005) show very high porosity at the top of the domain in Long Shot (about 0.54) and Cannikin (about 0.92). These values are unrealistically high and as such the profiles are scaled down such that the maximum porosity at the top does not exceed the cavity and chimney porosity of 0.4 
(Figure 4.5 [d through f]). Details of incorporating porosity profiles into the groundwater flow and transport model are presented in Section 5.
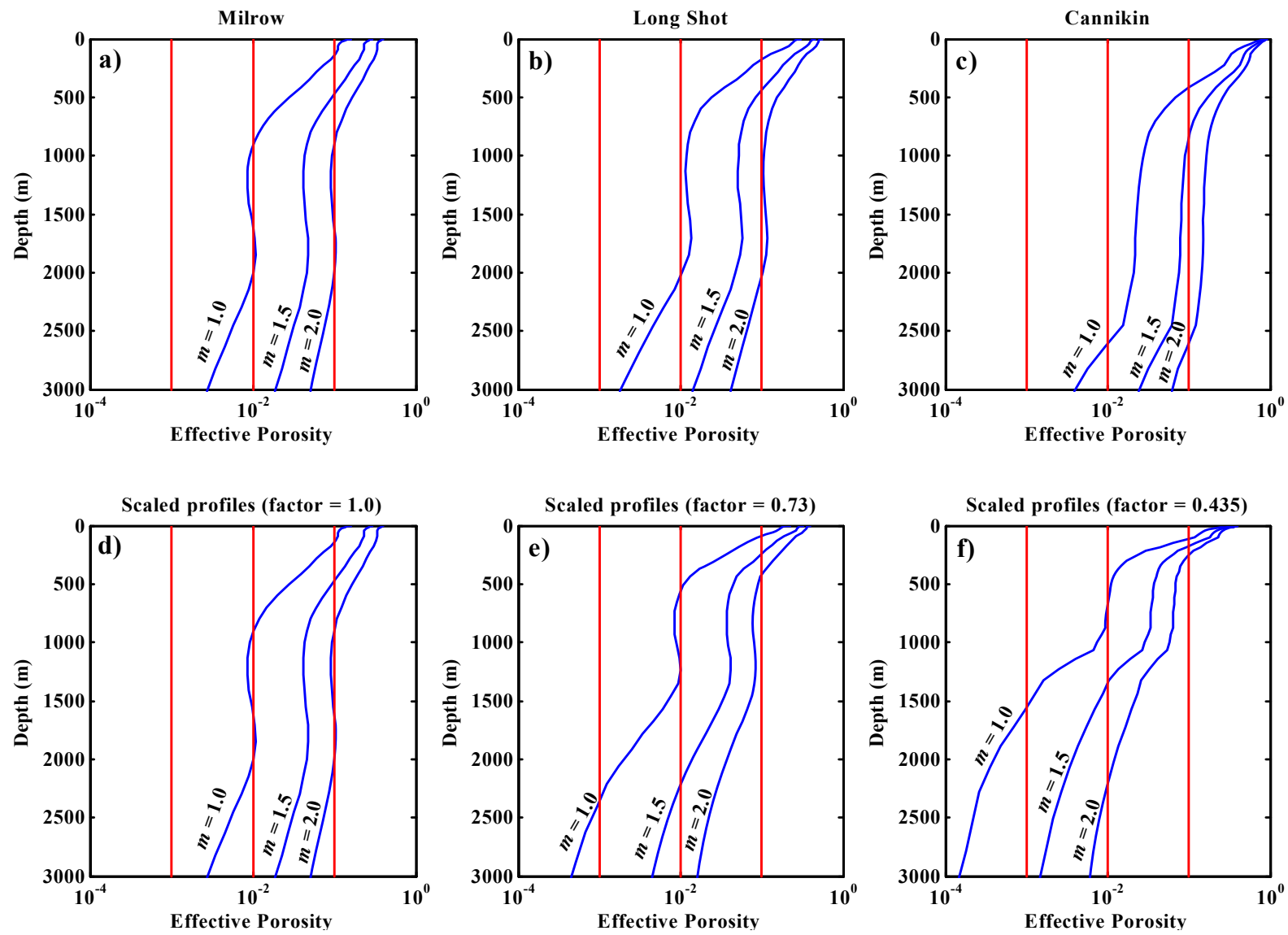

Figure 4.5. Effective porosity profiles provided by Unsworth et al. (2005) for the three sites (a, b, and $\mathrm{c}$ ), and scaled-down profiles to enforce a maximum porosity of 0.4 at the top (d, e, and f).

\subsubsection{Bathymetric Profiles}

The upper boundary of the model domain in Hassan et al. (2002) was the topographic (on island) and bathymetric (on sea) profiles extracted from topographic and bathymetric maps. von Huene et al. (1971) was used for bathymetric data in the 2002 models to define the boundary conditions for the seaward portion of the domain. Transects were extended into the ocean from each test location and the elevation of the seafloor was recorded at each point where the transect crossed a contour. Elevations for the land-surface portion of each domain are based on the Amchitka Island Map Atlas (Holmes and Narver, 1976).

The CRESP expedition mapped bathymetry north of Amchitka from about the 20-m depth to the 100-m isobath offshore of Cannikin and Long Shot, to a 2-m resolution using a multibeam echosounder (Johnson and Stewart, 2005). They note that the historical bathymetry is in general agreement with the newly acquired data. 
The high-resolution bathymetric data of Johnson and Stewart (2005) are compared to the bathymetric profiles used in Hassan et al. (2002). Figure 4.6 (a) displays the contouring of Johnson and Stewart's (2005) bathymetric data with transects representing the Long Shot and Cannikin models superimposed on the map. A comparison of the bathymetric profiles used to construct the upper model boundary in Hassan et al. (2002) to the profiles extracted from Johnson and Stewart's (2005) grid for the two models reveals little difference between them (Figure $4.6 \mathrm{~b}, \mathrm{c}$ ). Thus the bathymetry of von Huene et al. (1971) is verified for use in defining the upper model boundaries for Long Shot and Cannikin, and the geometry of the model domain used in the current study is the same as that in Hassan et al. (2002).

\subsection{Prior Densities}

A parameter's prior density summarizes the available information about the parameter before considering any newly collected data (Marshall et al., 2004). In many applications, a uniform prior distribution is assumed in the absence of prior information or knowledge about the parameter. In this case, the range of the parameter values must be wide enough to represent vague prior knowledge.

For the Amchitka models, the parameters of concern are the recharge and hydraulic conductivity, which impact the solution of the density driven flow problem and determine the transition zone location, salinity distribution, and head distribution. The other uncertain parameters in Hassan et al.'s (2002) models do not impact the solution to the flow problem and thus are not subject to MCMC updating. However, as indicated earlier, the uncertain effective porosity distribution used in Hassan et al. (2002) is replaced here with the uncertain effective porosity profiles (Figure 4.5) provided by Unsworth et al. (2005).

The input parameter distributions of $K$ and Rech used in the 2002 study are used here as the prior densities. These distributions were based on 1) a qualitative calibration (based on visual comparisons) using salinity and head data, 2) constraints on the ranges of the parameters to produce outputs that are not impacted by the domain boundaries, and 3) Amchitka-related literature values as well as literature values of parameters in similar environments.

The distributions of recharge, conductivity and recharge-conductivity ratio as implemented in the 2002 model (histograms) were fit with lognormal densities (Figure 4.7). It should be noted that conductivity and recharge were generated from their respective lognormal distributions, but the ratio $R / K$ was obtained by dividing the corresponding values of $R$ and $K$. That is the reason the lognormal fit to $R / K$ in Figure 4.7 is not as good as the fits to $R$ and $K$. However, the fits to $R / K$ are not used in the MCMC algorithm and only the individual parameter fits are used as prior distributions in the algorithm. These fits (the red lines) will form the basis for comparing the prior distribution to the posterior distribution obtained from MCMC. It is important here to emphasize that MCMC will update the $R$ and $K$ values only and the resulting $R / K$ posterior will be obtained from the pairs of $R$ and $K$ generated through the MCMC algorithm. 
a)
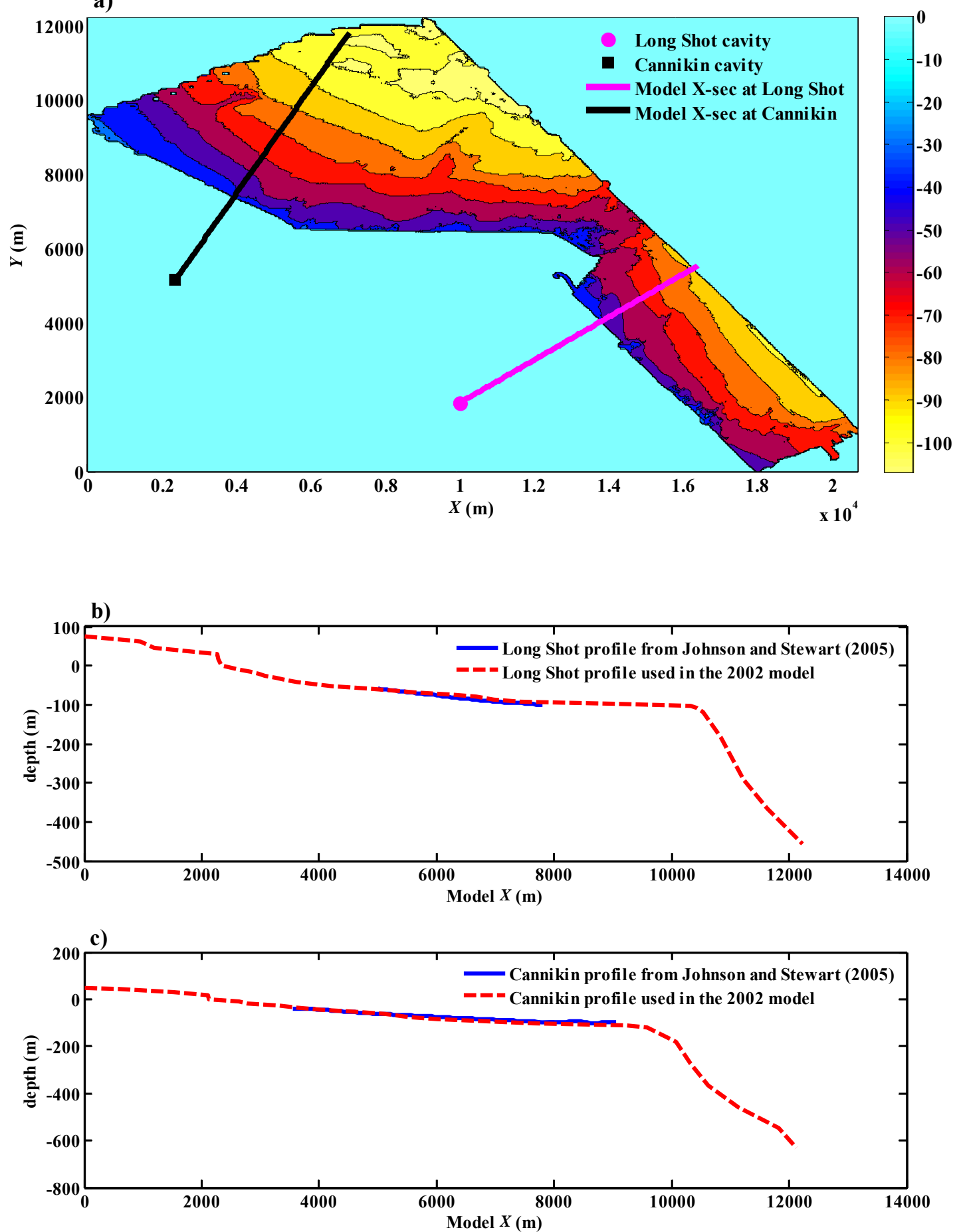

Figure 4.6. Two-dimensional map of bathymetric levels provided by Johnson and Stewart (2005) (a) and comparison between bathymetric profiles used in 2002 model and those extracted from the top map for Cannikin (b) and Long Shot (c). 

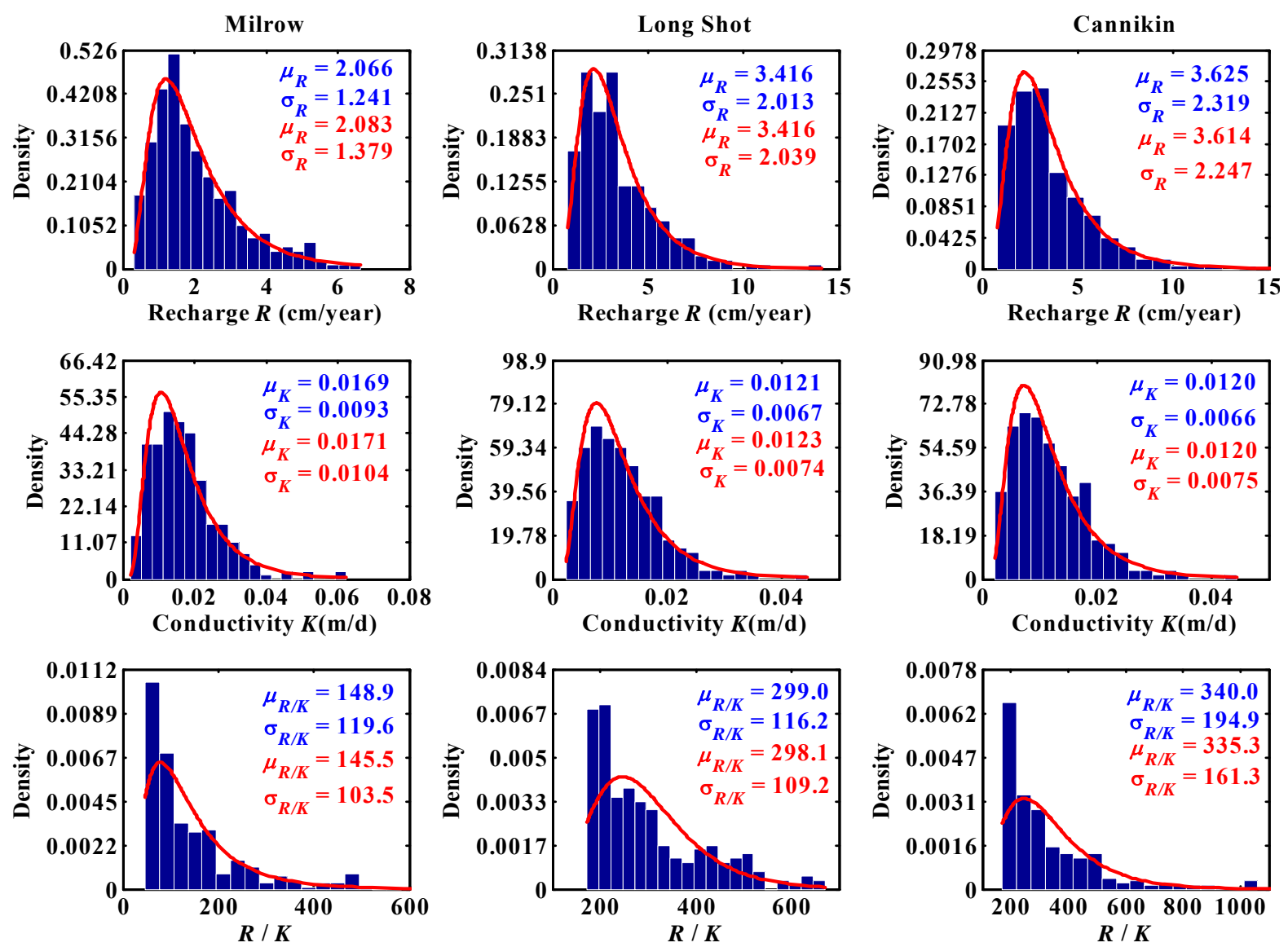

Figure 4.7. Prior distributions used in the current study. Blue histograms show the distributions used in Hassan et al. (2002) and red curves show the theoretical prior distributions that fit these histograms.

\subsection{Proposal Densities}

The adaptive Metropolis (AM) algorithm presented by Haario et al. (2001) and used to develop the posterior distributions of $R$ and $K$. At each iteration, the AM algorithm relies on a proposal density function that is updated with the information gained (i.e., the samples generated) from the posterior distribution. The proposal density is based on the estimated posterior covariance matrix of the parameters, which is computed at each iteration based on past iterations. For the Amchitka model, $R$ and $K$ are correlated, so a bivariate normal distribution is used as the proposal density function. This distribution has the form

$$
f\left(x_{1}, x_{2}\right)=\frac{1}{2 \pi \sigma_{1} \sigma_{2} \sqrt{1-\rho^{2}}} \exp \left[-\frac{\frac{\left(x_{1}-\mu_{1}\right)^{2}}{\sigma_{1}^{2}}-\frac{2 \rho\left(x_{1}-\mu_{1}\right)\left(x_{2}-\mu_{2}\right)}{\sigma_{1} \sigma_{2}}+\frac{\left(x_{2}-\mu_{2}\right)^{2}}{\sigma_{2}^{2}}}{2\left(1-\rho^{2}\right)}\right]
$$

where $\mu_{1}$ is the mean of $x_{1}, \sigma_{1}$ is the standard deviation of $x_{1}, \mu_{2}$ is the mean of $x_{2}, \sigma_{2}$ is the standard deviation of $x_{2}$, and $\rho$ is the correlation coefficient of $x_{1}$ and $x_{2}$. The covariance matrix for these normal bivariates can be written as 


$$
\operatorname{Cov}\left(x_{1}, x_{2}\right)=\left(\begin{array}{ll}
\sigma_{11} & \sigma_{12} \\
\sigma_{21} & \sigma_{22}
\end{array}\right)=\left(\begin{array}{cc}
\sigma_{1}^{2} & \rho \sigma_{1} \sigma_{2} \\
\rho \sigma_{1} \sigma_{2} & \sigma_{2}^{2}
\end{array}\right)
$$

Rewriting Equations (4.1) and (4.2) using the two parameters $R$ and $K$ yields

$$
\begin{gathered}
f(R, K)=\frac{1}{2 \pi \sigma_{R} \sigma_{K} \sqrt{1-\rho_{R K}^{2}}} \exp \left[-\frac{\frac{\left(R-\mu_{R}\right)^{2}}{\sigma_{R}^{2}}-\frac{2 \rho_{R K}\left(R-\mu_{R}\right)\left(K-\mu_{K}\right)}{\sigma_{R} \sigma_{K}}+\frac{\left(K-\mu_{K}\right)^{2}}{\sigma_{K}^{2}}}{2\left(1-\rho_{R K}^{2}\right)}\right] \\
\operatorname{Cov}(R, K)=\left(\begin{array}{cc}
\sigma_{R}^{2} & \rho_{R K} \sigma_{R} \sigma_{K} \\
\rho_{R K} \sigma_{R} \sigma_{K} & \sigma_{K}^{2}
\end{array}\right)
\end{gathered}
$$

As indicated in Equation (3.7) for the AM algorithm, this covariance assumes a fixed value for the first $i_{0}$ iterations and is subsequently updated. For the first 10,000 iterations ( $i \leq 10,000)$ of the MCMC algorithm, the proposed values of $K$ and $R\left(R^{*}\right.$ and $\left.K^{*}\right)$ are generated from Equation (4.3) using the $i^{\text {th }}$ parameter values as the mean and the fixed covariance obtained from the prior distributions as the covariance. That is, for $i \leq 10,000$,

$$
\begin{aligned}
& \begin{array}{l}
\mu_{R}=R^{i} \\
\mu_{K}=K^{i}
\end{array} \\
& \operatorname{Cov}(R, K)=\left(\begin{array}{cc}
(1.379)^{2} & 0.3437(1.379)(0.0104) \\
0.3437(1.379)(0.0104) & (0.0104)^{2}
\end{array}\right) \quad \text { for Milrow } \\
& \operatorname{Cov}(R, K)=\left(\begin{array}{cc}
(2.039)^{2} & 0.7695(2.039)(0.0074) \\
0.7695(2.039)(0.0074) & (0.0074)^{2}
\end{array}\right) \quad \text { for Long Shot } \\
& \operatorname{Cov}(R, K)=\left(\begin{array}{c}
(2.247)^{2} \\
0.6171(2.247)(0.0075)
\end{array}\right) \quad \text { for Cannikin }
\end{aligned}
$$

Subsequently, at iteration $i=10,001$, the covariance is computed from the first 10,000 values of $R$ and $K$. Similarly, for $i=10,002$, the covariance is computed from the first 10,001 values of $R$ and $K$ and so on. 
To reduce the computational burden of computing the covariance at each iteration, which becomes time consuming as $i$ increases, Equation (3.8) is used in a recursive manner and is implemented as follows

$$
\operatorname{Cov}_{i+1}=\frac{i-1}{i} \operatorname{Cov}_{i}+\frac{S_{d}}{i}\left[i\left(\begin{array}{cc}
\bar{R}^{i-1} \bar{R}^{i-1} & \bar{R}^{i-1} \bar{K}^{i-1} \\
\bar{K}^{i-1} \bar{R}^{i-1} & \bar{K}^{i-1} \bar{K}^{i-1}
\end{array}\right)-(i-1)\left(\begin{array}{cc}
\bar{R}^{i} \bar{R}^{i} & \bar{R}^{i} \bar{K}^{i} \\
\bar{K}^{i} \bar{R}^{i} & \bar{K}^{i} \bar{K}^{i}
\end{array}\right)+\left(\begin{array}{cc}
R^{i} R^{i} & R^{i} K^{i} \\
K^{i} R^{i} & K^{i} K^{i}
\end{array}\right)\right]
$$

where $\bar{R}^{i-1}$ for example is the average of all recharge, $R$, values generated from iteration $i=0$ to iteration $i-1$ (i.e., $\bar{R}^{i-1}=\frac{1}{i}\left[R^{0}+R^{1}+\ldots+R^{i-1}\right]$ ). The scaling factor, $S_{d}$, is adjusted during the simulation to provide a reasonable acceptance rate in the range from 30 to 70 percent.

\subsection{Likelihood Functions}

The likelihood function describes the probability that a given parameterized model is consistent with observed data. It is a function of the model and measures the fit between model predictions and observations. In statistical terms, the likelihood function is the joint probability density function of the data, considered as a function of the model parameter values. The data affect the posterior distribution through the likelihood function (Marshall et al., 2004).

The likelihood function used here is similar to that used by Bates and Campbell (2001) and Marshall el al. (2004) of the form

$$
P(\mathbf{D} \mid \Theta)=\frac{1}{2 \mu \sigma_{\varepsilon}^{2}} \exp \left\{-\frac{\sum_{j=1}^{N_{D}}\left[\mathbf{D}\left(\mathbf{x}_{j}\right)-M\left(\mathbf{G}, \mathbf{x}_{j} ; \boldsymbol{\Theta}\right)\right]^{2}}{2 \sigma_{\varepsilon}^{2}}\right\}
$$

where $P(\mathbf{D} \mid \Theta)$ is the likelihood function of the observed data vector $\mathbf{D}, \mathbf{D}\left(\mathbf{x}_{j}\right)$ is the observation or data value at domain location $\mathbf{x}_{j}, M\left(\mathbf{G}, \mathbf{x}_{j} ; \Theta\right)$ is the model output for location $\mathbf{x}_{j}, \mathbf{G}$ is the set of model input describing domain geometry, boundary conditions, and discretization, $N_{D}$ is the number of data points available for conditioning, and $\sigma_{\varepsilon}^{2}$ is the variance of the error terms. As stated above, this is the only place where the data influence the algorithm. In each MCMC iteration, the generated $R$ and $K$ values are used in the groundwater flow model and the resulting salinities and heads are then subtracted from their corresponding observations to yield the error term. These error terms are then used in Equation (4.7) to obtain $P(\mathbf{D} \mid \Theta)$, needed in Equation (3.9) to compute the acceptance probability, $\alpha$.

\subsection{Approach}

The steps involved in running the MCMC using the AM algorithm are shown in the flowchart of Figure 4.8. Iteration $i=0$ starts with initial arbitrary values for recharge and conductivity, $R^{0}$ and $K^{0}$. These could be either assumed or picked at random from the prior distributions. For a new iteration $i=1$, the first step is to compute the covariance at the 
current iteration based on the selected threshold $i_{0}$ and using either Equation (4.5) or (4.6). The proposal density, Equation (4.3), is then used to generate proposal values for recharge and conductivity, $R^{*}$ and $K^{*}$. This is illustrated schematically in Figure 4.9 for $K$. The proposal density (blue line) is centered at the current value $K^{0}$, which might be obtained from the prior distribution of $K$. Then $K^{*}$ is drawn at random from the proposal density.

Once current and proposed values for $R$ and $K$ are available, the groundwater flow model is run using $\left(R^{0}\right.$ and $\left.K^{0}\right)$ and $\left(R^{*}\right.$ and $\left.K^{*}\right)$ and the salinity and head distributions in the simulation domain are obtained in each case. The error terms are then evaluated by subtracting the model predictions from the observations, and these error terms are used in the likelihood function (Equation (4.7)) to obtain $P\left(\mathbf{D} \mid \Theta^{0}\right)=P\left(\mathbf{D} \mid R^{0}, K^{0}\right)$ and $P\left(\mathbf{D} \mid \Theta^{*}\right)=P\left(\mathbf{D} \mid R^{*}, K^{*}\right)$. Using the current and proposed values, the densities $P\left(R^{0}\right), P\left(K^{0}\right), P\left(R^{*}\right)$, and $P\left(K^{*}\right)$ are computed from the prior distributions of $R$ and $K$. The acceptance probability, $\alpha$, is then computed using Equation (3.9). A random number is then drawn from a uniform $[0,1]$ distribution and is compared to $\alpha$, to determine whether to accept the proposed values. If $R^{*}$ and $K^{*}$ are accepted, then the first sample $\left(R^{1}, K^{1}\right)$ takes the values $\left(R^{*}, K^{*}\right)$, otherwise it equals $\left(R^{0}, K^{0}\right)$. This completes the first iteration of the AM algorithm.

For $i=2$, new proposed values, $R^{*}$ and $K^{*}$, are generated from the bivariate normal distribution of Equation (4.3) using $R^{1}$ and $K^{1}$ as means and the same covariance used in iteration $i=1$, since this still falls below the threshold $i_{0}$. This process continues until $I=i_{0}+1$, at which time the covariance needed for the proposal density is computed from the values $\left[\left(R^{0}, K^{0}\right),\left(R^{1}, K^{1}\right),\left(R^{2}, K^{2}\right), \ldots,\left(R^{i_{0}}, K^{i_{0}}\right)\right]$. For $i \geq i_{0}+2$, the recursive equation (4.6) is used to obtain the covariance.

The groundwater flow model needs to run every iteration using the proposed values $R^{*}$ and $K^{*}$ to obtain the head and salinity distribution in the simulation domain and subsequently the errors needed for the likelihood function. Given that the MCMC algorithms usually require tens of thousands of iterations to reach the stationary posterior distribution of model parameters, it becomes prohibitive from a computational point of view to run a groundwater model for these many iterations.

To overcome this obstacle, the 2002 model realizations were studied and it was found that the terms needed in the likelihood function, namely the sum of squared errors $\sum_{j=1}^{N_{D}}\left[\mathbf{D}\left(\mathbf{x}_{j}\right)-M\left(\mathbf{G}, \mathbf{x}_{j} ; \boldsymbol{\Theta}\right)\right]^{2}$ and the error variance $\sigma_{\varepsilon}^{2}$, are highly correlated to the rechargeconductivity ratio, $R / K$. As a result, the groundwater model itself does not need to run at all for the MCMC algorithm since the existing realizations (300 for Milrow, 240 for Long Shot, and 260 for Cannikin) were sufficient for establishing the relationships between $R / K$ and the sum of squared errors and between $R / K$ and the error variance. In the case of Long Shot, the range of $R / K$ was not large enough to elucidate the relationship, and thus 50 additional realizations were run using the original model to establish these relationships. 


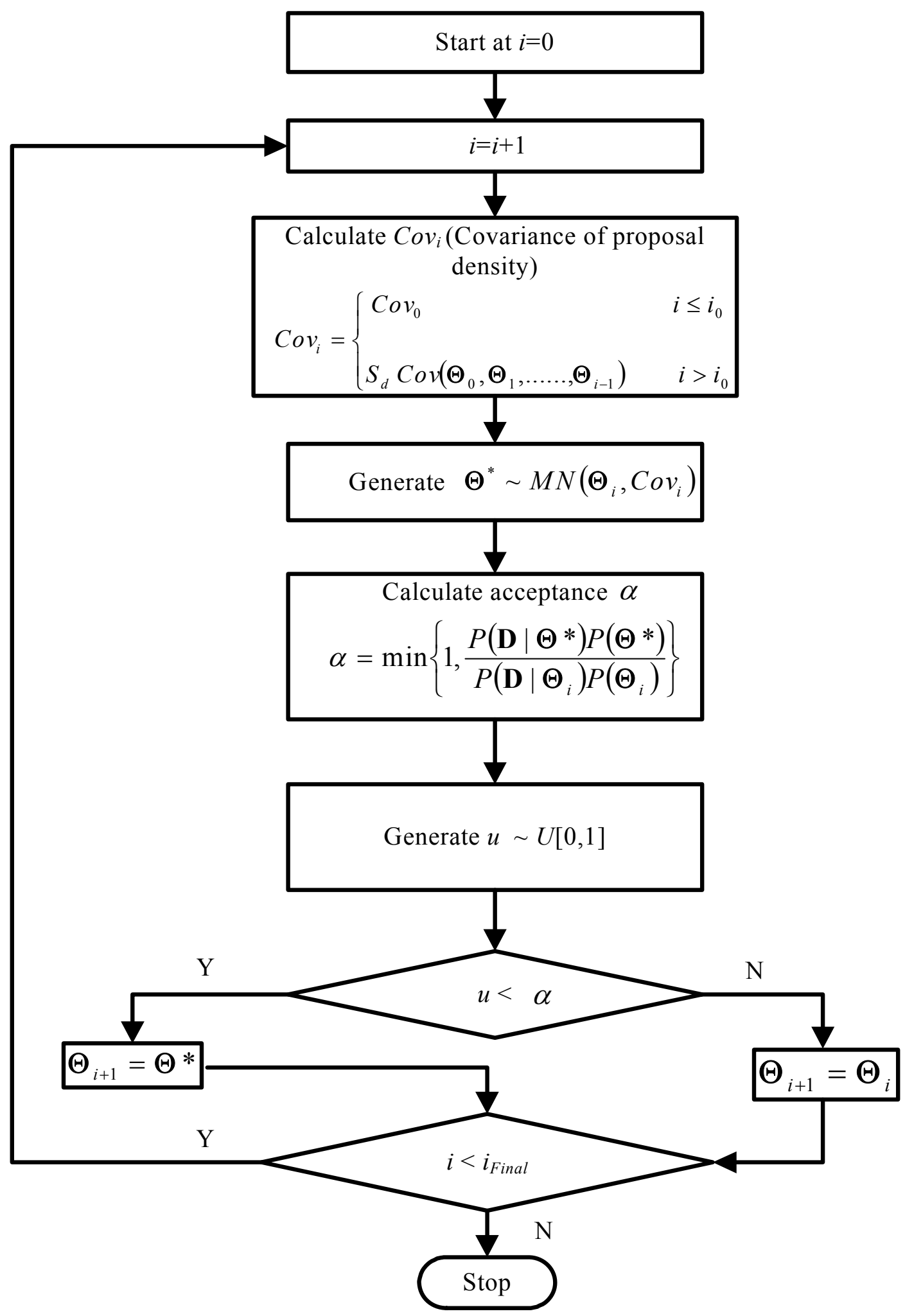

Figure 4.8. A flowchart showing the steps of the MCMC algorithm implemented in this study. 


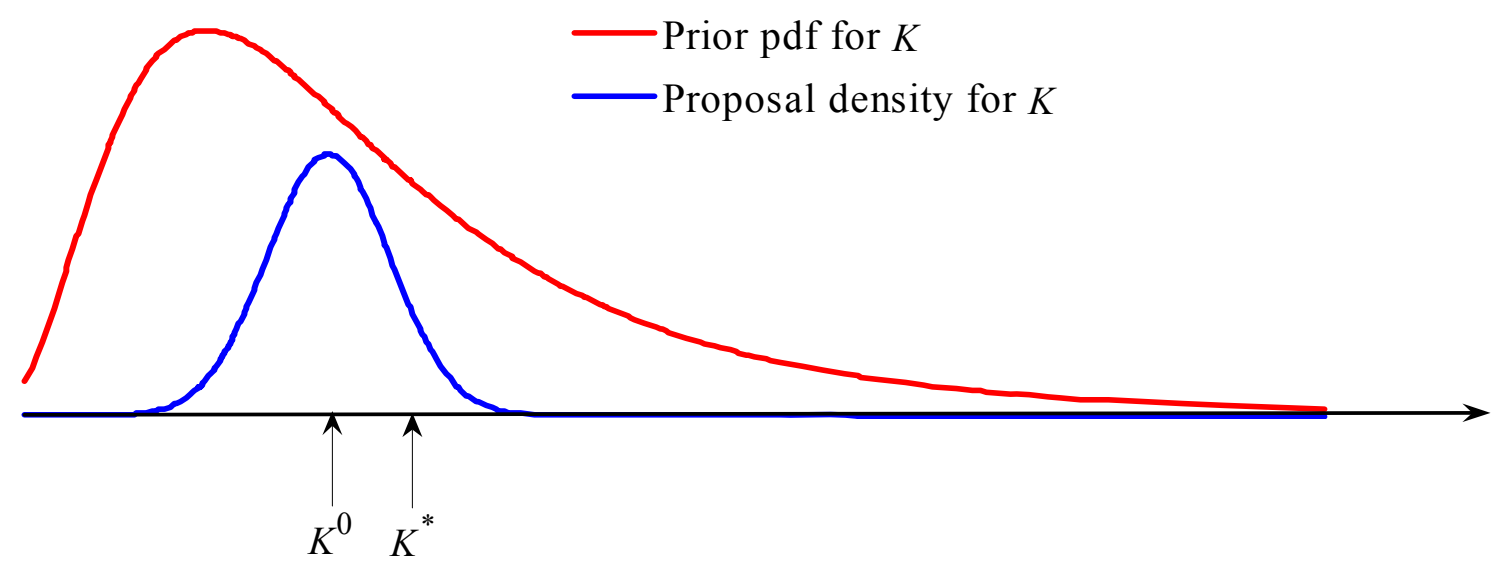

Figure 4.9. A schematic showing the selection of the initial value, $K^{0}$, from the prior distribution, and the construction of the proposal density with the initial value as its mean. The proposed value, $K^{*}$, is then selected at random from the proposal density.

The original 300 realizations of Hassan et al. (2002) Milrow model were used to generate the relationship between $R / K$ and the sum of squared errors, $\sum_{j=1}^{N_{D}}\left[\mathbf{D}\left(\mathbf{x}_{j}\right)-M\left(\mathbf{G}, \mathbf{x}_{j} ; \Theta\right)\right]^{2}$ (Figure 4.10a), and the relationship between $R / K$ and the error variance, $\sigma_{\varepsilon}^{2}$ (Figure 4.10b). Very clear relationships exist ion both cases. This allows the use of either look-up tables or high-degree polynomials relating the two quantities to $R / K$. Then, during the running of MCMC algorithm, when $R^{*}$ and $K^{*}$ values are proposed, the ratio $R^{*} / K^{*}$ is computed and the corresponding sum of squared errors and error variance terms are obtained from the look-up tables or the fitted polynomials.

Very clear and well established relations also exist between the error terms and $R / K$ in the 2002 Long Shot (Figure 4.11) and the Cannikin (Figure 4.12) models. This eliminated the need to run the groundwater model for tens of thousands of iterations, thereby making the implementation of this Bayesian framework for the Amchitka models feasible. 

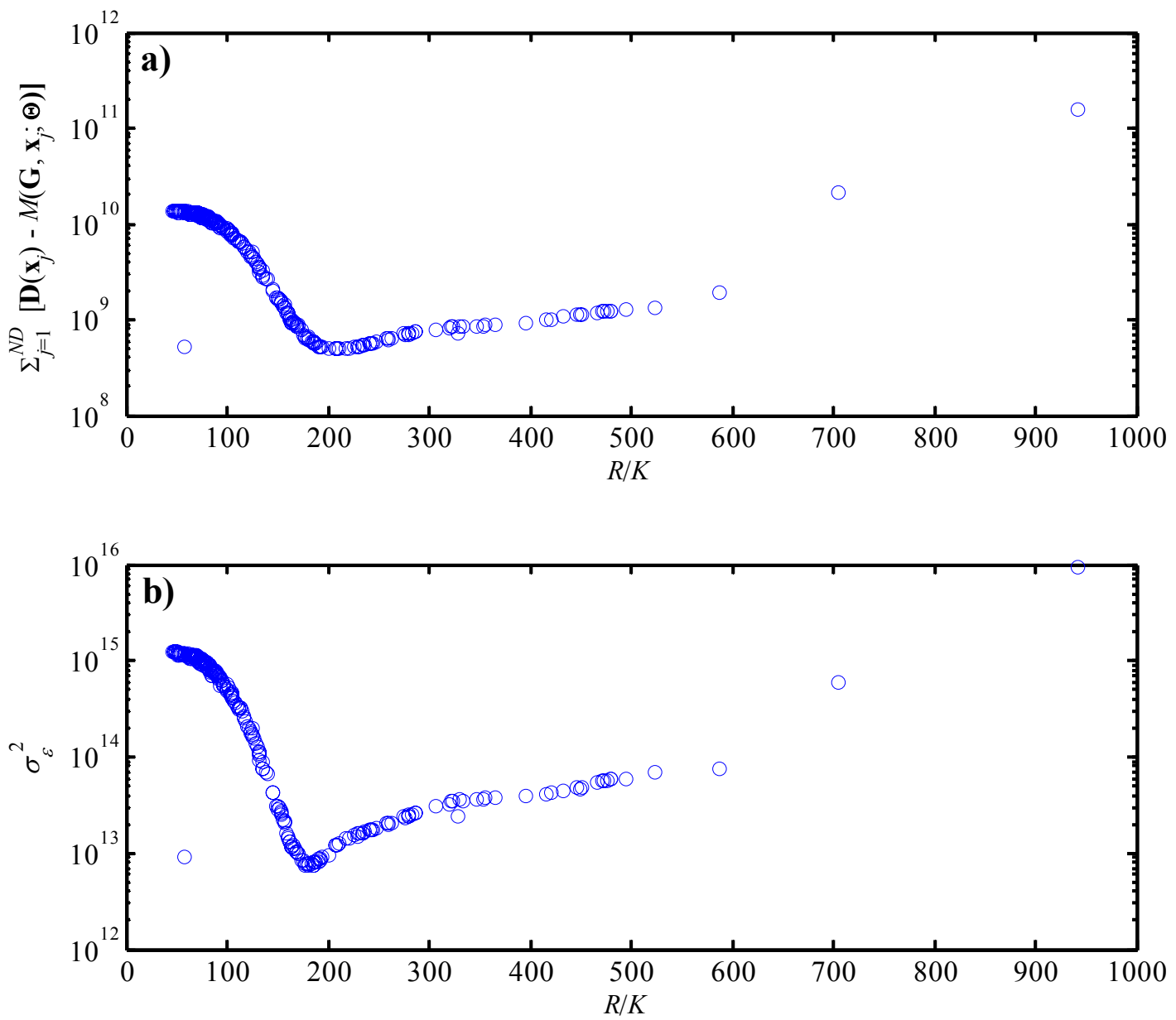

Figure 4.10. Relationship between $R / K$ and the sum of squared errors (a) and the error variance (b) used in the likelihood function for Milrow. 

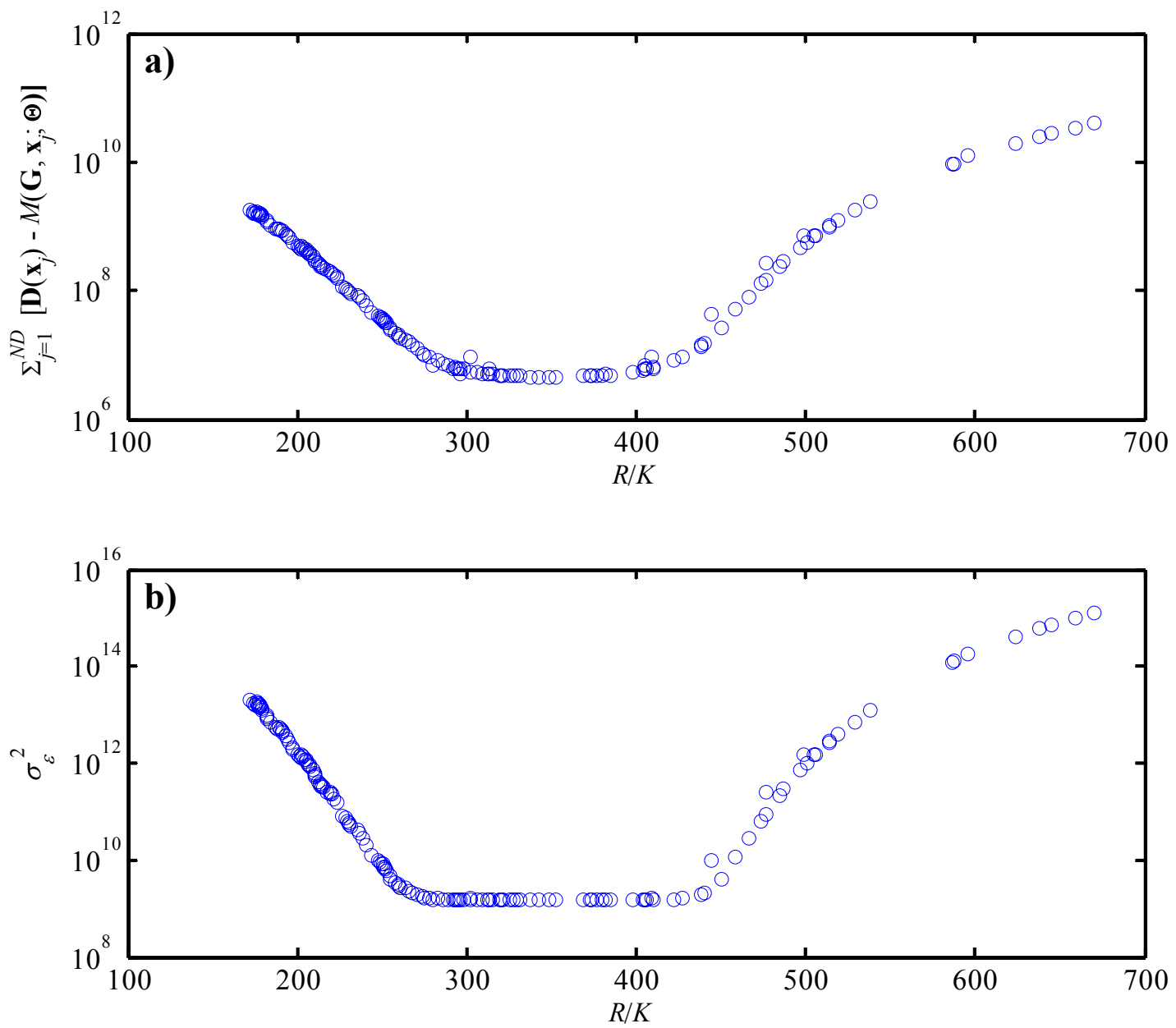

Figure 4.11. Relationship between $R / K$ and the sum of squared errors (a), and the error variance (b) used in the likelihood function for Long Shot. 

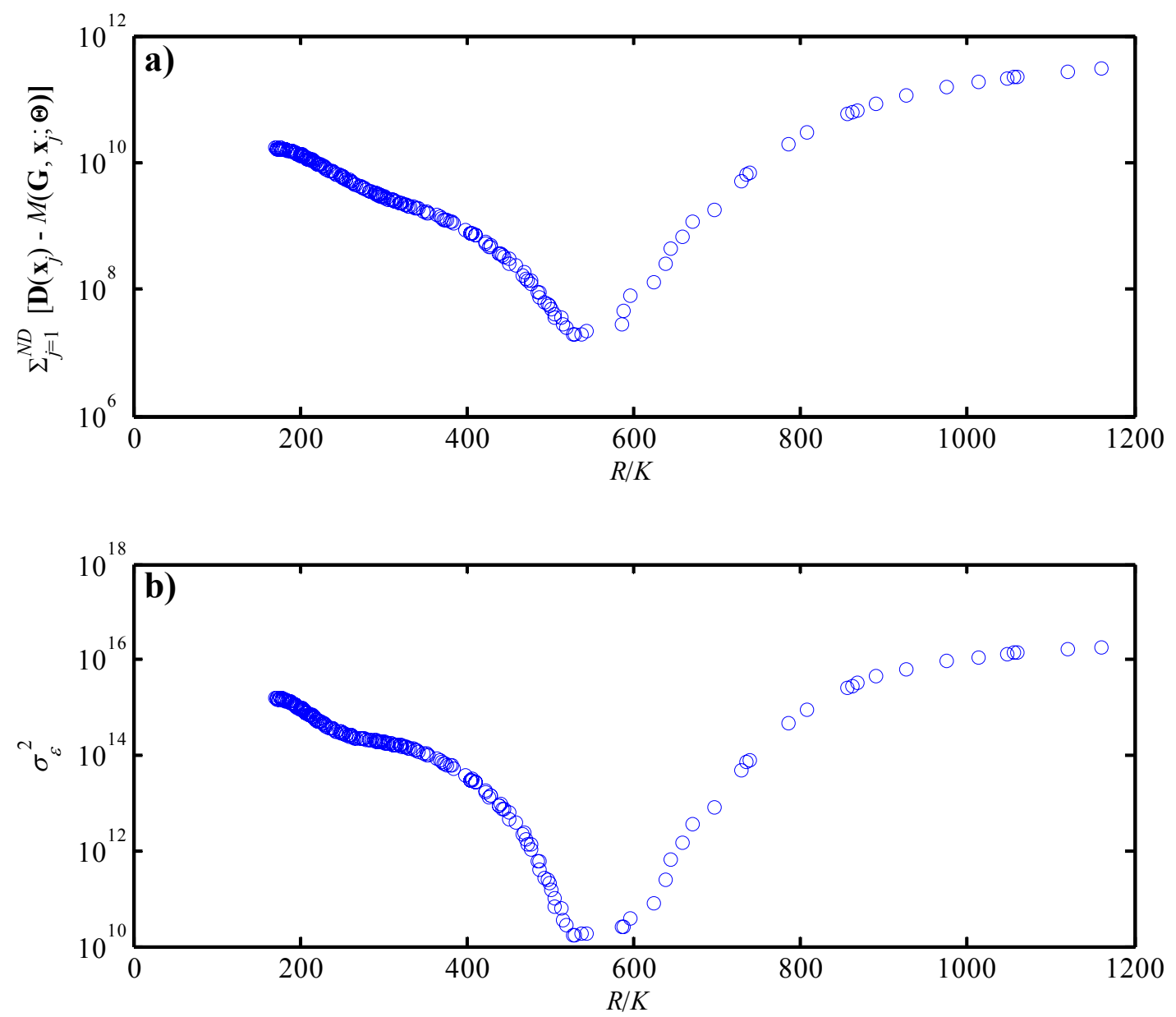

Figure 4.12. Relationship between $R / K$ and the sum of squared errors (a), and the error variance (b) used in the likelihood function for Cannikin.

\subsection{Diagnostic Checks}

Once initialized, the Metropolis algorithm is allowed to continuously sample until the Markov chain created by the algorithm has converged to a stationary probability. At this point, the Metropolis algorithm samples are considered samples from the posterior distribution. One of the critical issues in implementing MCMC methods is how to determine whether the generated samples have converged to the posterior. The main question here is how many iterations the Markov chain should be allowed to "warm up" before the sampled values can be considered samples of the posterior. Because there is no general technique to determine the number of burn-in or warm-up iterations necessary for convergence, some form of analysis should be performed to assess convergence. Such analyses are known as convergence diagnostic checks.

Several diagnostic tests are performed to test the convergence of the AM algorithm. First, the stability of the moments (i.e., mean and variance) of generated parameters is established. Second, different numbers of burn-in iterations and total number of iterations are 
analyzed to determine the reasonable number of iterations to run and the reasonable burn-in number. Finally, the stability of the joint posterior distribution of $R$ and $K$ is established.

Figures 4.13 to 4.15 exhibit the stability of the moments for the generated parameters, $R, K$, and $R / K$, respectively, using the Milrow model as an example and conditioning parameter distributions on a combined salinity and MT data set (see discussion in Sections 4.7.1 and 4.7.2). In each figure, subplot (a) displays the parameter trace, subplots (b), and display (c), the parameter mean and variance, respectively, as functions of the number of iterations, and subplot (d) displays the lognormal density estimate for the marginal posterior distribution. It can be seen from the parameter traces that a reasonable mixing rate was achieved with several jumps, deviations, or excursions away from the zone of highest posterior density.
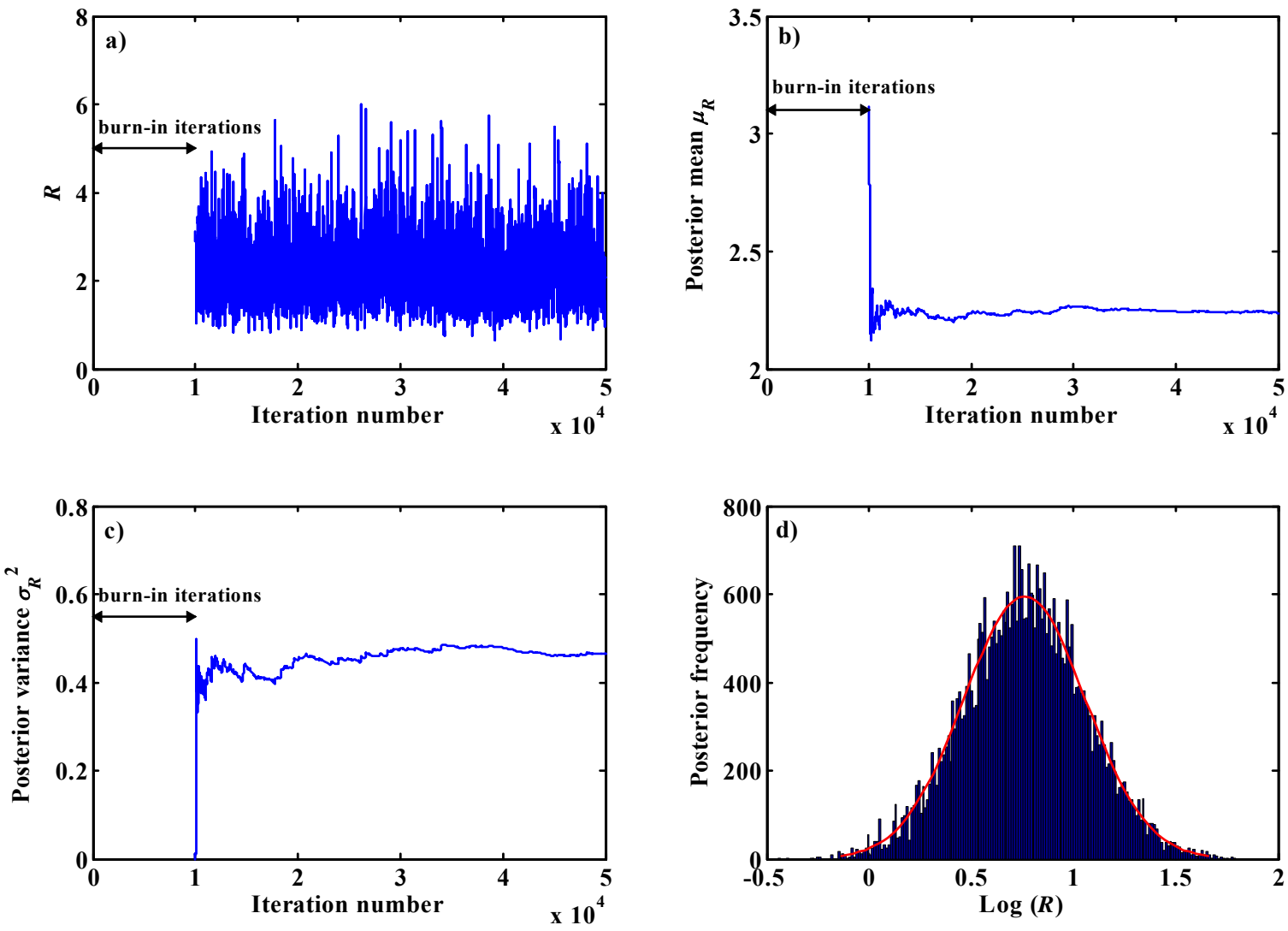

Figure 4.13. Milrow MCMC diagnostics for $R$ : (a) parameter trace, (b) posterior mean trace, (c) posterior variance trace, and (d) histogram of $\log (R)$ with a fitted posterior frequency curve (red line). 

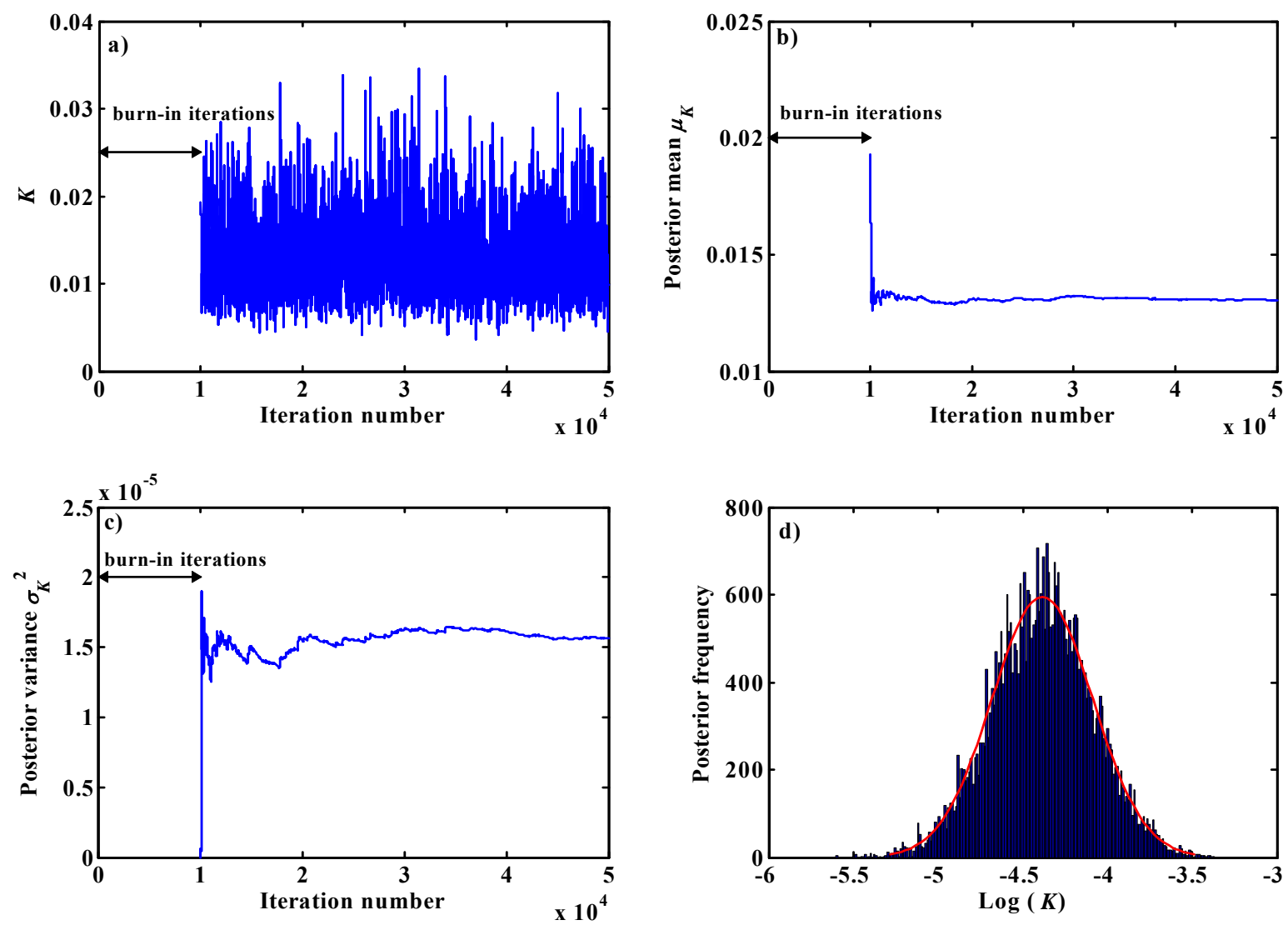

Figure 4.14. Milrow MCMC diagnostics for $K$ : (a) parameter trace, (b) posterior mean trace, (c) posterior variance trace, and $(d)$ histogram of $\log (K)$ with a fitted posterior frequency curve (red line).

The posterior mean and variance traces are stable after about 40,000 iterations, which indicates that the $\mathrm{MCMC}$ algorithm has converged to the limiting posterior distribution. The generated samples for $R, K$, and $R / K$ are nicely fitted with a lognormal posterior distribution (Figures 4.13d, 4.14d, and 4.15d). These fits for $R$ and $K$ are the marginal posterior distributions for the two parameters.

To provide a more quantitative check on the stability of the moments and the effect of both the number of burn-in iterations and the total number of iterations, Table 4.4 displays the mean and variance of parameter traces for different combinations of these two numbers. These results are obtained for the Milrow model and used as a combined concentration data set (see Sections 4.7.1 and 4.7.2) for conditioning. The table shows that although the moments (mean and variance) change as the total number of iterations increases, the change is relatively small and the statistics are reasonably stable at 100,000 iterations with 10,000 burn-in iterations. As a result, unless stated otherwise, 100,000 MCMC iterations are used in all subsequent analysis with 10,000 burn-in iterations. 

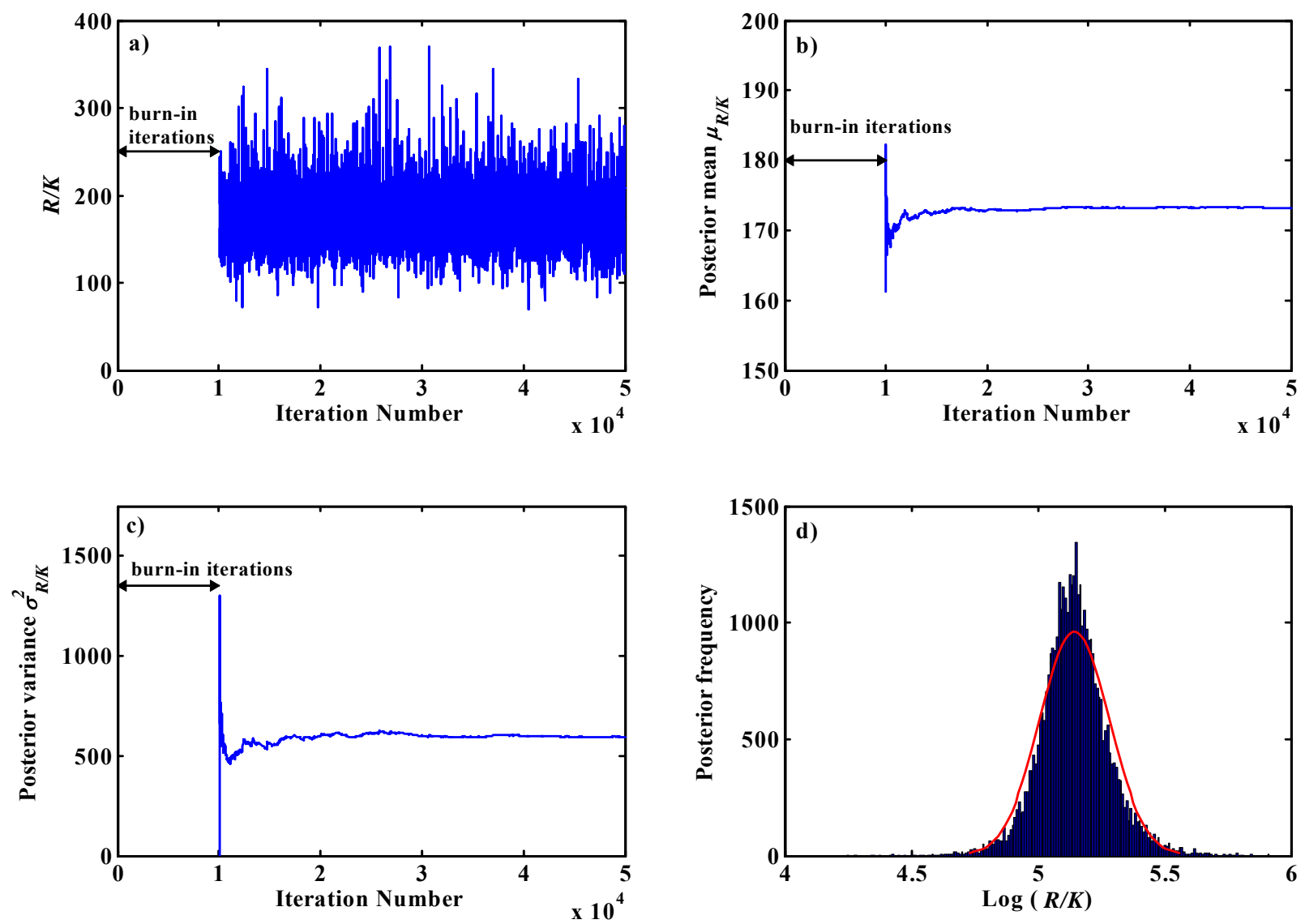

Figure 4.15. Milrow MCMC diagnostics for $R / K$ : (a) parameter trace, (b) posterior mean trace, (c) posterior variance trace, and (d) histogram of $\log (R / K)$ with a fitted posterior frequency curve (red line).

The joint posterior density of $R$ and $K$ is shown in Figure 4.16a and the values of $R$ are plotted against the values of $K$ in Figure 4.16b. The strong positive correlation is evident from the figure. This has important implications for the posterior distribution of the rechargeconductivity ratio, as will be seen in the results section. The positive correlation means that high values of $K$ are associated with high values of $R$ and vice versa. The result is a small range for the recharge-conductivity ratio, which reduces the uncertainty in the transition zone location.

The results of the diagnostic checks indicate that the algorithm is stable and that the resulting posterior distributions are stationary. These results are expected in the sense that conditioning on the data that determine the transition zone location leads to a narrow range of $R / K$, and as will be seen in the results, the posterior density for this ratio has a high peak and a small spread. The ability of the algorithm to capture the correlation is an advantage of the MCMC and the simultaneous parameter updating implemented in the AM method. 
Table 4.4. MCMC diagnostic analysis of the effect of the number of burn-in iterations and the total number of iterations on the mean and variance of generated parameters.

\begin{tabular}{|c|c|c|c|c|}
\hline \multicolumn{5}{|c|}{$\mu_{R}$} \\
\hline \multirow{2}{*}{$\begin{array}{c}\text { Number of } \\
\text { burn-in } \\
\text { iterations }\end{array}$} & 50,000 & 100,000 & 150,000 & 200,000 \\
\hline 5,000 & 2.4510 & 2.4896 & 2.4945 & 2.5205 \\
\hline 10,000 & 2.4626 & 2.4969 & 2.4993 & 2.5248 \\
\hline 15,000 & 2.4764 & 2.5046 & 2.5043 & 2.5291 \\
\hline 20,000 & 2.4894 & 2.5113 & 2.5083 & 2.5327 \\
\hline
\end{tabular}

\begin{tabular}{|c|c|c|c|c|}
\hline \multicolumn{5}{|c|}{$\sigma_{R}^{2}$} \\
\hline \multirow{2}{*}{$\begin{array}{c}\text { Number of } \\
\text { burn-in } \\
\text { iterations }\end{array}$} & 50,000 & 100,000 & 150,000 & 200,000 \\
\cline { 2 - 5 } & \multicolumn{4}{|c|}{ Total number of iterations } \\
\hline 5,000 & 1.1255 & 1.2079 & 1.2282 & 1.2607 \\
\hline 10,000 & 1.1604 & 1.2275 & 1.2415 & 1.2712 \\
\hline 15,000 & 1.1868 & 1.2418 & 1.2511 & 1.2787 \\
\hline 20,000 & 1.2122 & 1.2545 & 1.2593 & 1.2853 \\
\hline
\end{tabular}

\begin{tabular}{|c|c|c|c|c|}
\hline \multicolumn{5}{|c|}{$\mu_{K}$} \\
\hline \multirow{2}{*}{$\begin{array}{c}\text { Number of } \\
\text { burn-in } \\
\text { iterations }\end{array}$} & 50,000 & 100,000 & 150,000 & 200,000 \\
\cline { 2 - 5 } & Total number of iterations \\
\hline 5,000 & 0.0123 & 0.0125 & 0.0125 & 0.0126 \\
\hline 10,000 & 0.0123 & 0.0125 & 0.0125 & 0.0126 \\
\hline 15,000 & 0.0124 & 0.0125 & 0.0125 & 0.0126 \\
\hline 20,000 & 0.0125 & 0.0126 & 0.0125 & 0.0126 \\
\hline
\end{tabular}

\begin{tabular}{|c|c|c|c|c|}
\hline \multicolumn{5}{|c|}{$\sigma_{K}^{2}$} \\
\hline \multirow{2}{*}{$\begin{array}{c}\text { Number of } \\
\text { burn-in } \\
\text { iterations }\end{array}$} & 50,000 & 100,000 & 150,000 & 200,000 \\
\cline { 2 - 5 } & \multicolumn{4}{|c|}{ Total number of iterations } \\
\hline 5,000 & $2.796 \mathrm{E}-05$ & $2.986 \mathrm{E}-05$ & $2.975 \mathrm{E}-05$ & $3.010 \mathrm{E}-05$ \\
\hline 10,000 & $2.873 \mathrm{E}-05$ & $3.030 \mathrm{E}-05$ & $3.002 \mathrm{E}-05$ & $3.031 \mathrm{E}-05$ \\
\hline 15,000 & $2.952 \mathrm{E}-05$ & $3.071 \mathrm{E}-05$ & $3.027 \mathrm{E}-05$ & $3.050 \mathrm{E}-05$ \\
\hline 20,000 & $3.010 \mathrm{E}-05$ & $3.099 \mathrm{E}-05$ & $3.043 \mathrm{E}-05$ & $3.061 \mathrm{E}-05$ \\
\hline
\end{tabular}

\begin{tabular}{|c|c|c|c|c|}
\hline \multicolumn{5}{|c|}{$\mu_{R / K}$} \\
\hline \multirow{2}{*}{$\begin{array}{c}\text { Number of } \\
\text { burn-in } \\
\text { iterations }\end{array}$} & 50,000 & 100,000 & 150,000 & 200,000 \\
\hline 5,000 & 202.676 & 202.207 & 202.723 & 203.029 \\
\hline 10,000 & 203.141 & 202.387 & 202.858 & 203.136 \\
\hline 15,000 & 202.746 & 202.181 & 202.745 & 203.061 \\
\hline 20,000 & 202.498 & 202.052 & 202.688 & 203.029 \\
\hline
\end{tabular}

\begin{tabular}{|c|c|c|c|c|}
\hline \multicolumn{5}{|c|}{$\sigma_{R / K}^{2}$} \\
\hline \multirow{2}{*}{$\begin{array}{c}\text { Number of } \\
\text { burn-in } \\
\text { iterations }\end{array}$} & 50,000 & 100,000 & 150,000 & 200,000 \\
\hline 5,000 & 1524.90 & 1355.30 & 1442.80 & 1558.80 \\
\hline 10,000 & 1589.30 & 1374.80 & 1458.30 & 1573.20 \\
\hline 15,000 & 1566.20 & 1352.30 & 1447.40 & 1568.40 \\
\hline 20,000 & 1542.50 & 1330.00 & 1437.40 & 1564.50 \\
\hline
\end{tabular}

a) Joint posterior density of $R$ and $K$ using 100,000 iteration

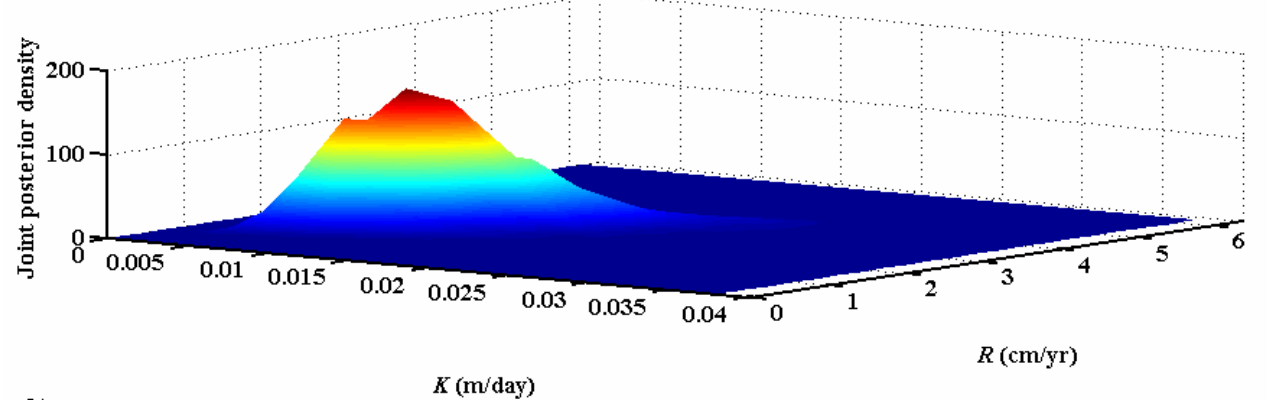

b)

$K$ (m/day)

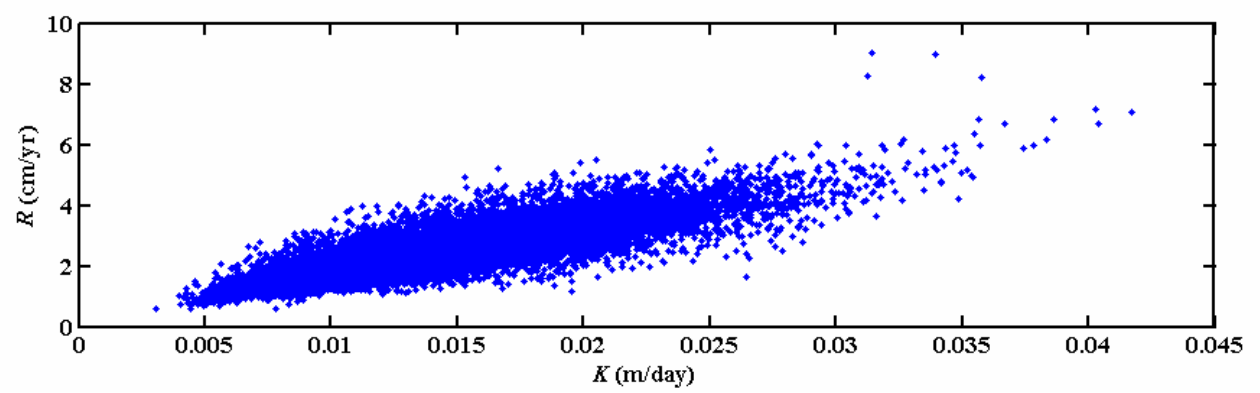

Figure 4.16. Joint posterior density of $R$ and $K$ (a) and the parameter trace plot (b) showing the strong positive correlation. 


\subsection{Results}

The approach outlined in the previous section was applied to the three sites. The results are the posterior distributions conditioned on prior knowledge and available data including the original salinity and head data and new data gathered by CRESP. The salinity data consisted of a few data points at each site and the head data also consisted of a small number of measurements. The MT data provided a profile along a large portion of the domain that could be subjectively converted to salinity data providing as many as 500 points for the model elements. Therefore, the effect of salinity and head data vanishes when the MT data are added. Two questions pose themselves immediately. The first is how to use the MTinterpreted transition zone location and convert it to salinity for comparison with the model output. This is briefly discussed in Section 4.7.1. The second question is how to combine the different data sets. There are many ways to combine the three data sets and use them in the MCMC conditioning. These different approaches are briefly discussed in Section 4.7.2. Detailed analyses, discussion, and sensitivity runs are presented in the Appendix.

\subsubsection{Converting MT Data to Salinity Data}

As described above, the MT data indicated the location of the upper and lower bounds of the transition zone along transects through the three sites. The upper line of the transition zone calculated in the inversion models of Unsworth et al. (2005) is modeled as the bottom of the freshwater area, whereas the lower bound of the transition zone gives the starting position of the seawater concentration area. Conversions of the MT-based profiles to salinities were not reported by Unsworth et al. (2005) for Milrow and Long Shot, though two computed salinity profiles were presented for one point along the Cannikin transect. The two computed profiles differ from one another throughout the transition zone because they were calculated using different porosity-depth profiles, so they are used here to guide application of the MT data rather than as model input.

The MT data resulted in a transition zone location much deeper than that identified from the salinity profile at UAe- 2 at Milrow. Figure 4.17 compares the salinity profile at UAe-2 (the red line) to the transition zone boundaries mapped from the resistivity profile resulting from the inversion of MT data (Unsworth et al., 2005) at Milrow. The transition zone resulting from the MT data is given by the two dashed black lines denoted as A and B in the figure, whereas the transition zone resulting from the salinity profile at UAe- 2 ends at the dotted red line denoted as Line 1. The two transition zones are consistent below line B where any point below line B lies in the saltwater layer for both data sets. However, above line B there is little consistency between the salinity values in the two data sets.

Several factors may contribute to the lack of consistency and should be considered when deciding how to use the MT data in model calibration. The salinity data can be in error, a possibility considered more likely for Cannikin and Long Shot than for Milrow (see discussion in Hassan et al., 2002). Since the salinity data are much more limited spatially than the MT data, they will have a smaller role in the calibration.

The MT measurements, as well as inversion process, introduce smoothing to the profiles that could contribute to inconsistency with the water chemistry data. The synthetic inversion study presented by Unsworth et al. (2005), as part of their extensive model evaluation process, demonstrates that smoothing has an effect. In that example, the "true" 
transition zone layer occurs between 1,400 and 2,300 $\mathrm{m}$, but applying the inflection points used to identify the transition zone, the smoothed inversion model would indicate a transition zone from about 700 to $1,500 \mathrm{~m}$. The excellent match shown between the Milrow MT data and a calculated resistivity profile (Unsworth et al., 2005) relies on seawater salinities at a depth of $1,500 \mathrm{~m}$, in contrast to the inflection-determined transition zone base of $1,700 \mathrm{~m}$, and salinities in excess of $10,000 \mathrm{mg} / \mathrm{L}$ at depths shallower than the $900 \mathrm{~m}$ inflection-based top of the transition zone. Again, smoothing may be the cause of the discrepancy.

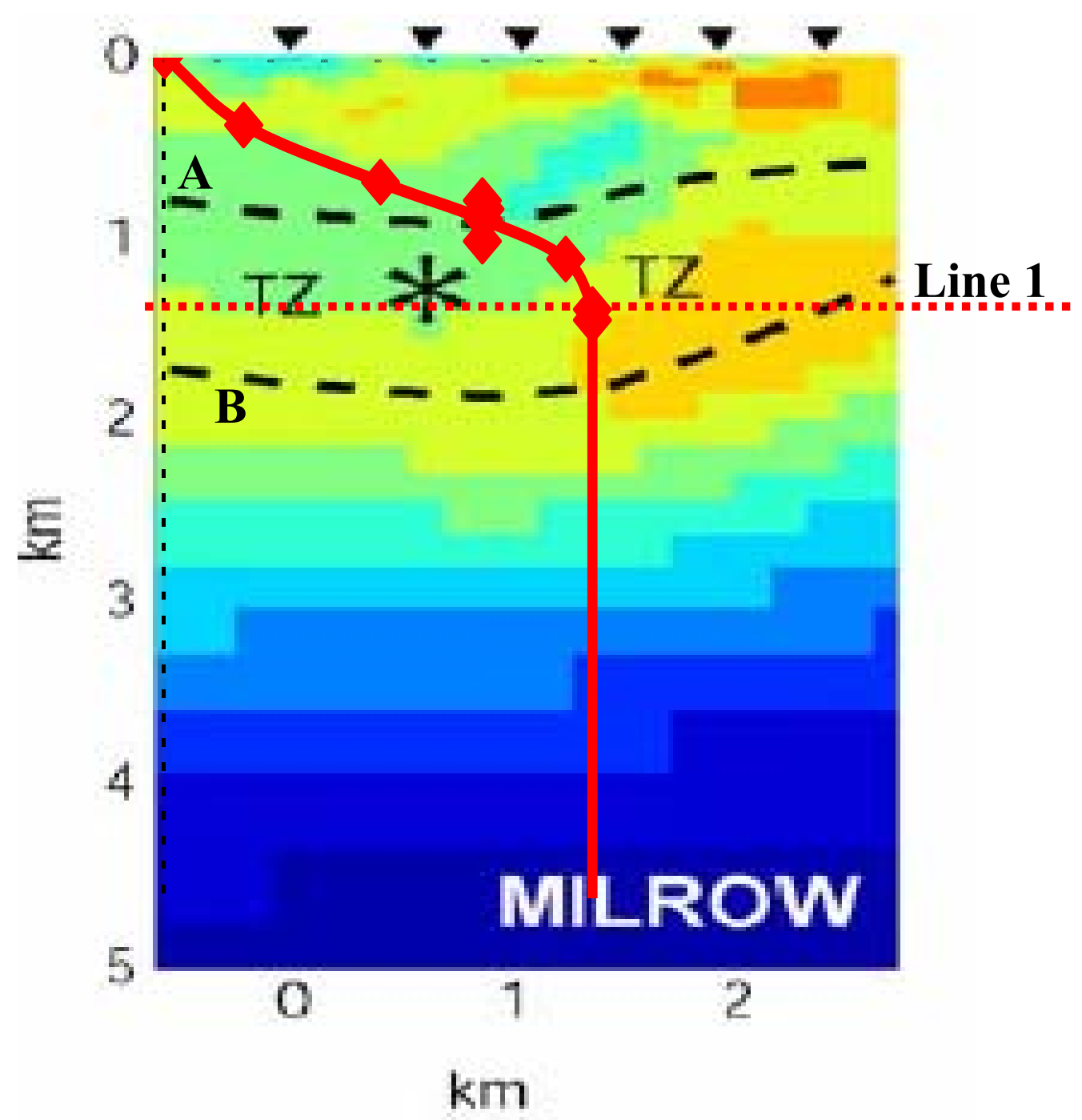

Figure 4.17. Resistivity map and interpreted transition zone boundaries (Lines A and B) from Unsworth et al. (2005) with the salinity profile from UAe-2 at Milrow (red line) superimposed. Line 1 indicates the beginning of seawater per the salinity data.

There may also be inconsistency between the sensitivity of the MT measurements and groundwater model interpretations of the transition zone. The groundwater models assume freshwater concentration above the transition zone. It may be that the MT measurements 
require a threshold value of water salinity to be achieved prior to registering as an overall resistivity decrease, given the other contributors to the resistivity measurement (such as resistivity of the rock). Thus the MT interpretations may not be resolved to the scale of salinities considered in the groundwater model, thus introducing some uncertainty. The bottom line is that the top of the transition zone identified from the MT data may not be the same as the line above which all the water is at freshwater concentrations (i.e., $\mathrm{Cl}$ concentrations of 0 to $50 \mathrm{mg} / \mathrm{L}$ ).

Four options were considered for applying the MT data for model calibration. The first option is to use the top and bottom of the transition zone identified by Unsworth et al. (2005) based on the geophysical inversion of the MT data (i.e., Lines A and B) as two lateral lines of freshwater and saltwater concentrations, respectively (Figure 4.18a). In this case, the model's cells or elements that are crossed by line A are assigned a salinity value equal to zero (freshwater concentration), and those that are crossed by line B are assigned a saltwater concentration value, $C_{\max }=18,100 \mathrm{mg} / \mathrm{L}$ chloride concentration.

The second option is to fill all the domain elements above line A with freshwater concentration and all the elements below line B with seawater concentration (Figure 4.18b). It should be noted that the domain thickness is $4,000 \mathrm{~m}$, so the elements below $3,000 \mathrm{~m}$ that are not shown in the figure are also assigned the maximum seawater concentration. The third option involves assigning the model elements that are crossed by line A freshwater concentration and assigning seawater concentration to the elements crossed by line $\mathrm{B}$ and all the elements below it. This is shown in Figure 4.19a. The last option is the reverse of the third option, where the elements crossed by or above line A are assigned freshwater concentration and only elements crossed by line B are assigned seawater concentration (Figure 4.19b). In Figures 4.18 and 4.19, the finite-element mesh with the white background has no data, nor do the elements in between lines A and B.

Option 3 is selected here for applying the MT data to the groundwater models. The groundwater chemistry data and MT data agree that below line B, groundwater salinity is at seawater concentration, even if there is some uncertainty above it. Applying freshwater concentrations along the upper transition zone boundary interpreted from the MT data, but not filling the overlying domain, honors the MT interpretation along the transect, but does not overly weight the upper profile given the observations of salinities higher than freshwater in the freshwater portion of the Milrow profile.

The Appendix presents sensitivity analyses to examine the impact of the different options discussed above on the results of the MCMC conditioning. In the analysis presented in subsection 4.7.3, the MT data set is converted to concentration data using the third option displayed in Figure 4.19a. 

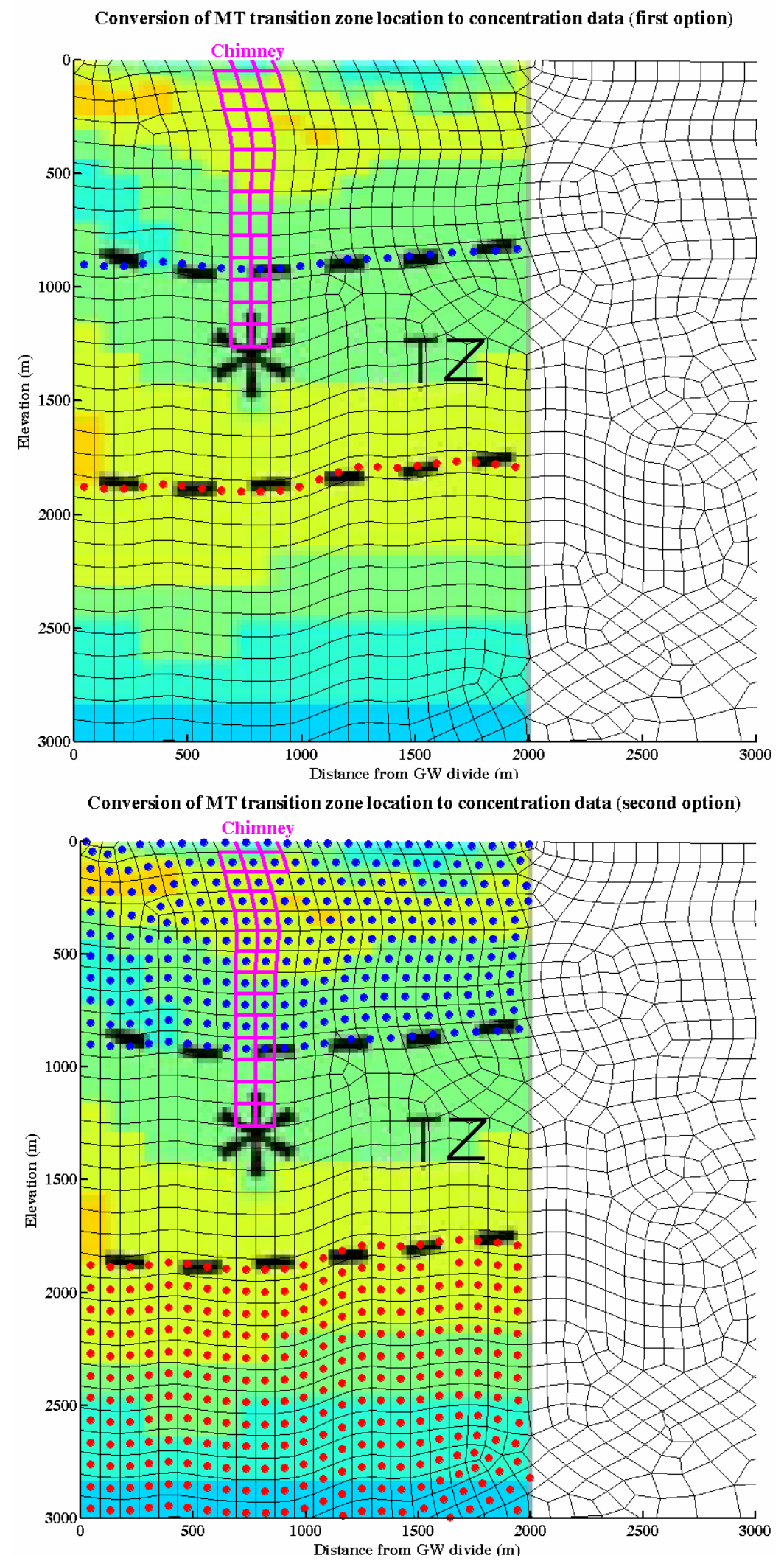

Figure 4.18. First and second options for converting MT to concentration data at Milrow. Red circles denote $C_{\max }$ and blue circles denote freshwater concentration 

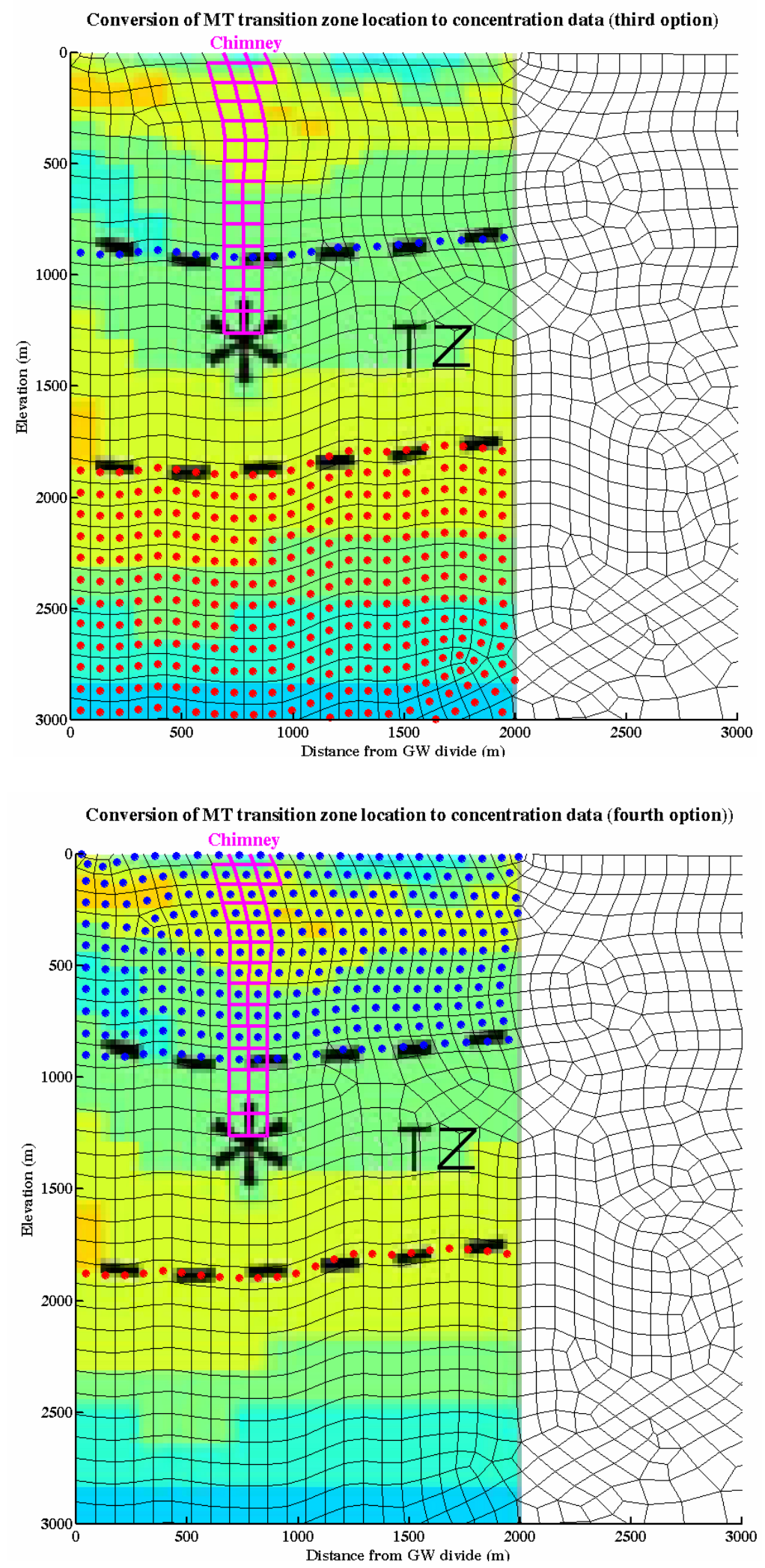

Figure 4.19. Third and fourth options for converting MT to concentration data at Milrow. Red circles denote $C_{\max }$ and blue circles denote freshwater concentration. 


\subsubsection{Combining Data Sets}

A number of alternatives exist for combining the salinity, MT, and hydraulic head data sets. The approach selected here is to combine the water chemistry and MT-converted (see previous section for the conversion of MT data to salinity) datasets into one salinitybased dataset, and consider the head data separately. Both the water salinity measurements and the MT-interpreted salinity provide information on the same system parameter, though the abundance of MT-derived data overwhelms the water chemistry data. A sequential conditioning approach is applied. The combined salinity-MT data set is used for conditioning and developing posterior distributions for the model parameters. These posterior distributions are then used as the prior distributions for conditioning on the hydraulic head data, leading to a final set of posterior distributions. Other approaches of data set combinations and conditioning sequence were evaluated and are presented in the Appendix.

\subsubsection{MCMC Conditioning Results}

At each of the three sites, the MT-converted concentrations are combined with the salinity data available from UAe-2 at Milrow, EH-5 at Long Shot, and UAe-1 at Cannikin and then used along with the prior parameter distributions to develop the first posterior. This posterior is then considered a prior and is used along with the head data available from UAe-2 at Milrow, the clustered wells at Long Shot, and UAe-1, UA-1, and HTH-1 at Cannikin to develop final parameter posterior distributions that are used in the flow and transport modeling. The results of this analysis for the three sites are described in the following.

\subsubsection{Milrow}

Figure 4.20 exhibits the MCMC conditioning results for Milrow. In the figure, $P(R)$, $P(K)$, and $P(R / K)$ denote the prior distributions taken as the distributions used in the 2002 model. $P\left(R \mid D_{1}\right), P\left(K \mid D_{1}\right)$, and $P\left(R / K \mid D_{1}\right)$ denote the first posterior obtained after conditioning on the combined salinity and MT-converted concentrations. Lastly, $P\left(R \mid D_{2}\right)$, $P\left(K \mid D_{2}\right)$, and $P\left(R / K \mid D_{2}\right)$ denote the final posterior distributions obtained using $P\left(R \mid D_{1}\right)$, $P\left(K \mid D_{1}\right)$, and $P\left(R / K \mid D_{1}\right)$ as the prior distributions when conditioning on the head data.

The recharge prior and posterior distributions are plotted in Figure 4.20a. After the first conditioning on the combined concentration data set (MT-converted concentrations and the chloride concentrations from UAe-2) the resulting posterior (red line) is shifted toward higher recharge values with less spread than the prior (blue line). The range of recharge values is slightly reduced from 0.2 to $6 \mathrm{~cm} / \mathrm{yr}$ to 0.5 to $6 \mathrm{~cm} / \mathrm{yr}$. The peak of the distribution has remained the same but its location has moved from a recharge value of about $1.2 \mathrm{~cm} / \mathrm{yr}$ to $1.95 \mathrm{~cm} / \mathrm{yr}$ (i.e., the distribution mode has changed). Subsequent conditioning on the heads yields a noticeable increase in the peak of the density function (compare red and green curves) and as a result, a significant decrease in the spread ( $\sigma_{R}$ decreased from 1.107 to 0.703 after head conditioning). 

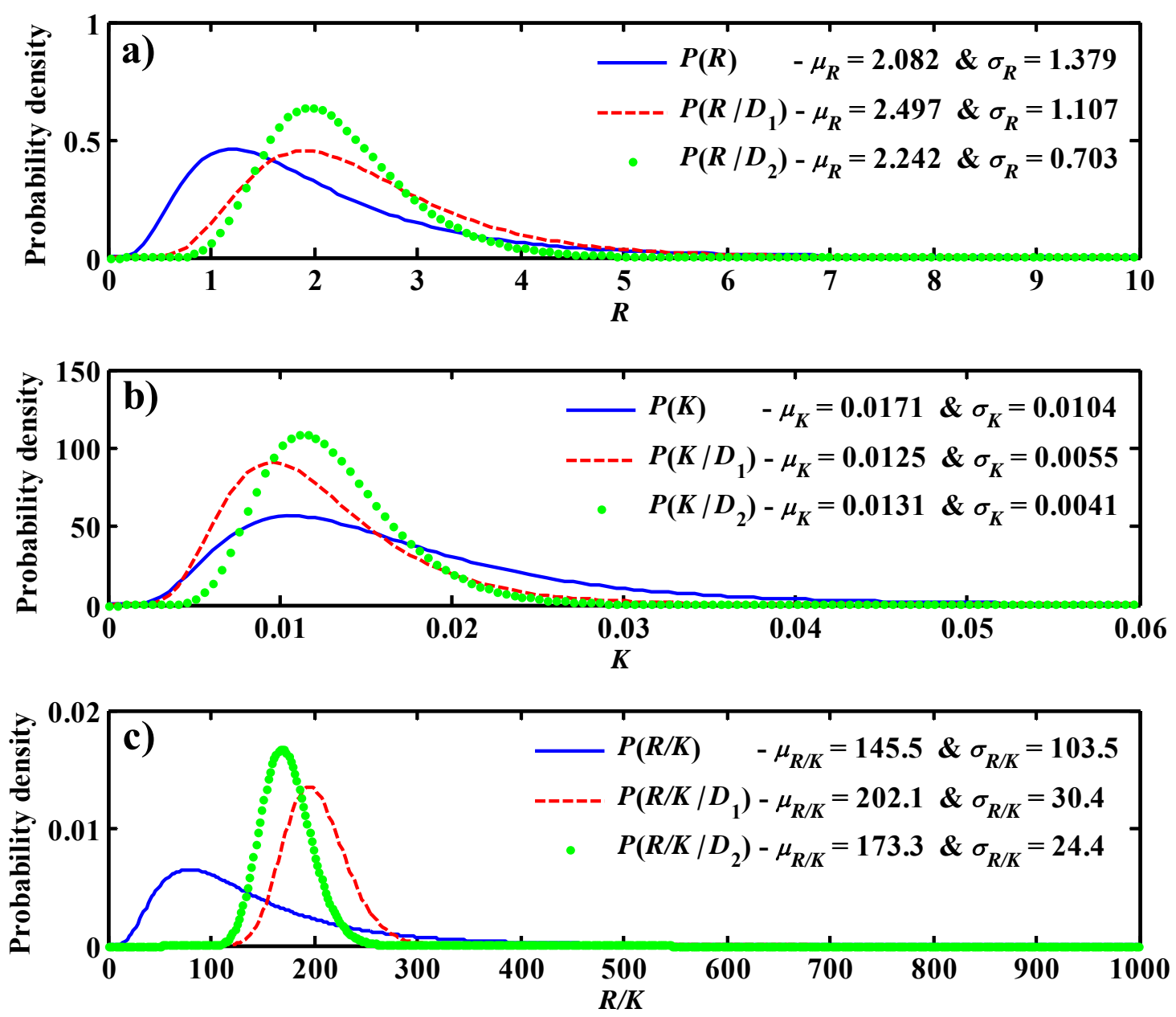

Figure 4.20. Results of the sequential conditioning approach for Milrow, where $D_{1}$ is the concentration data set (salinity at UAe-2 and MT-converted concentrations) and $D_{2}$ is the head data at UAe-2.

Conditioning on the combined concentration data yields a much narrower distribution for $K$ than its prior (Figure 4.20b). The range of possible conductivity values is reduced from $[0.002$ to $0.05 \mathrm{~m} / \mathrm{d}$ ] to $[0.002$ to $0.03 \mathrm{~m} / \mathrm{d}$ ]. The peak of the distribution is slightly shifted toward a lower conductivity value (i.e., the mode of the distribution has decreased) but with a higher density than the prior distribution. So in effect, the tails of the prior distribution are trimmed after the first conditioning on the combined concentration data set and the peak of the density function is increased. When conditioning is performed sequentially on the head data, a slight shift toward higher conductivity values occurs as can be seen in comparing $P\left(K \mid D_{1}\right)$ to $P\left(K \mid D_{2}\right)$. The peak also increased and $\sigma_{K}$ decreased from $5.5 \times 10^{-3}$ to $4.1 \times 10^{-3} \mathrm{~m} / \mathrm{d}$.

The impact of conditioning through MCMC is greatest for the recharge-conductivity ratio as shown in Figure 4.20c. Conditioning on the combined concentration data greatly reduces the density spreading and increases the highest density value more than twofold. In addition, the conditioning shifts the distribution toward higher $R / K$ values with the peak 
density occurring at a value of 200 compared to a value of about 79 in the prior distribution. This makes sense, as the data set is mainly controlled by the MT-converted concentrations. The MT data indicate a deeper transition zone than that indicated by the salinity data, which qualitatively guided the prior selection in the 2002 model. The range of possible values for this ratio is tightened, where it was [5 to 400] in the prior distribution and [120 to 300] in the posterior. When the head data are used in a sequential manner to condition the distribution, the final $R / K$ posterior (green curve) is shifted toward lower ratios and the peak density increases. The standard deviation of the distribution dramatically decreases after the first conditioning (from 103.5 to 30.4 ) and further decreases after the second conditioning (from 30.4 to 24.4$)$.

Conditioning on salinity and MT data yields large $R / K$ values and a deeper transition zone than indicated by the heads. When we subsequently condition on heads, the transition zone moves up and becomes shallower to honor the head data and $R / K$ gets a little smaller (moving $R / K$ posterior to the left). When looking at the three data sets individually (see Appendix), it can be seen that salinity data require lower $R / K$ values (shallow transition zone) than heads, and heads require lower values than MT data. So when the MT-converted data set is used along with the salinity data, the $R / K$ distribution is pushed to the highest values because of the dominance of the MT data, but when, subsequently, the attempt is made to accommodate heads, they pull the $R / K$ posterior back to a location that gives a slightly shallower transition zone.

Note that the posterior distributions plotted in Figure 4.20a and $\mathrm{b}$ are the marginal posterior distributions for $R$ and $K$, respectively. It is clear from Figure 4.20 that despite being a small data set, the heads have a noticeable effect on the marginal posterior distributions of $R$ and $K$, and a more pronounced effect on the posterior distribution of $R / K$. Also, the posterior distributions are closer to being normal, especially for $R / K$.

The above results are based on the theoretical prior and posterior fitted to the original 300 realizations and the MCMC-generated 90,000 values (100,000 iterations minus the 10,000 burn-in iterations), respectively. It is useful to compare the actual values of $R$ and $K$ for three cases (Figure 4.21). The figure displays (a) the $300 R$ - $K$ pairs of the 2002 model, (b) the 90,000 pairs generated by MCMC, and (c) the 2,000 pairs (used in flow and transport modeling) selected at random out of the 90,000 pairs. The plots also have the line of equal $R / K$ plotted on the data for the $R / K$ value with the highest prior or posterior density (i.e., the mode of the prior or posterior distribution).

The posterior pairs show a very strong positive correlation, which was not present in the prior pairs. The range of $R / K$ is very tight around the 170 value (Figure $4.21 \mathrm{~b}$ ), whereas in the prior, the range had a long tail toward the higher values of $R / K$ of Figure $4.21 \mathrm{a}$. The prior data had conductivity values reaching $0.06 \mathrm{~m} / \mathrm{d}$, whereas the 2,000 pairs to be used in the revised model have a maximum conductivity of about $0.032 \mathrm{~m} / \mathrm{d}$. For recharge, the prior data have a maximum recharge of about $6.5 \mathrm{~cm} / \mathrm{yr}$, whereas the 2,000 pairs have a maximum of about $6 \mathrm{~cm} / \mathrm{yr}$.

The 2,000 values of recharge and conductivity extracted from MCMC (Figure 4.21c) are the values used in the revised flow and transport modeling for Milrow. These 2,000 pairs are sampled from the 90,000 MCMC output using a stratified sampling approach (presented in more detail in Section 5). Because the $R-K$ pairs that have high values in Figure 4.21c have 
very low density compared to the lower values or $R$ and $K$, these values are not sampled as frequently. Combined with these 2,000 $R-K$ pairs, the porosity profiles of Unsworth et al. (2005) are used in the revised modeling. This is discussed in Section 5.
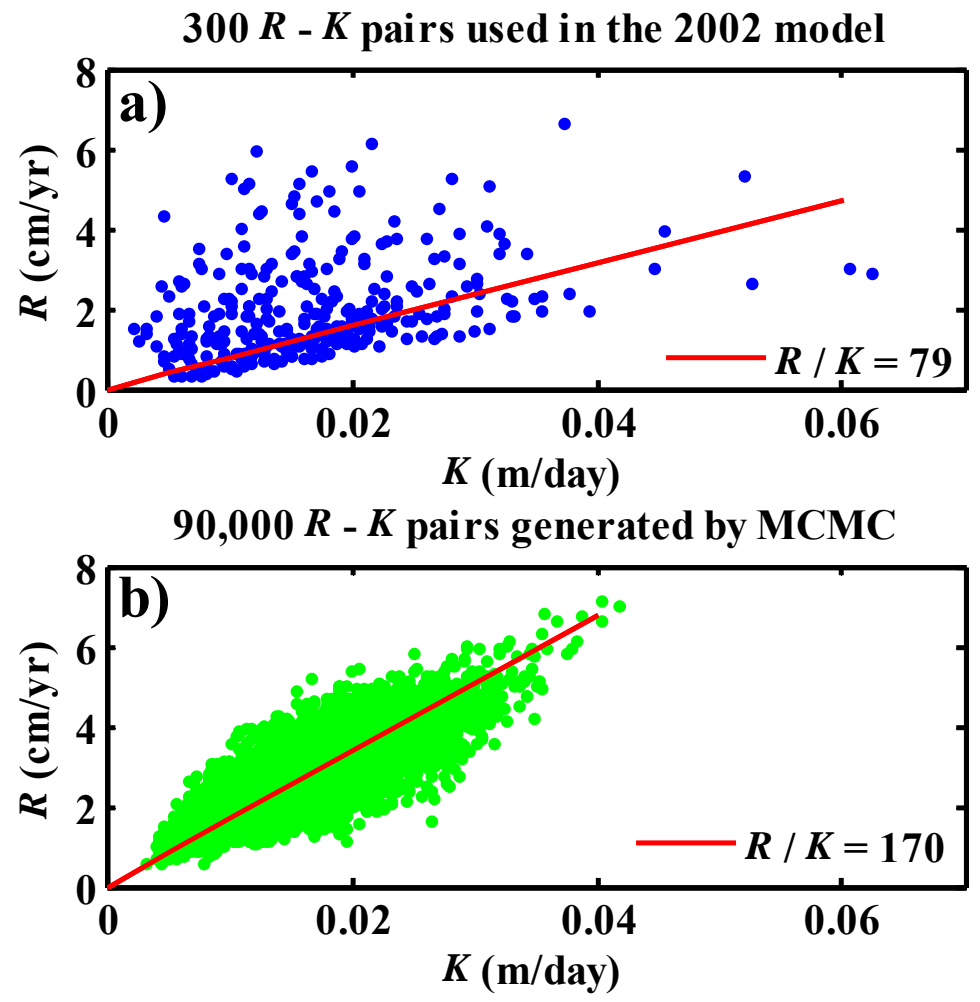

$2000 R-K$ pairs to be used in the revised model

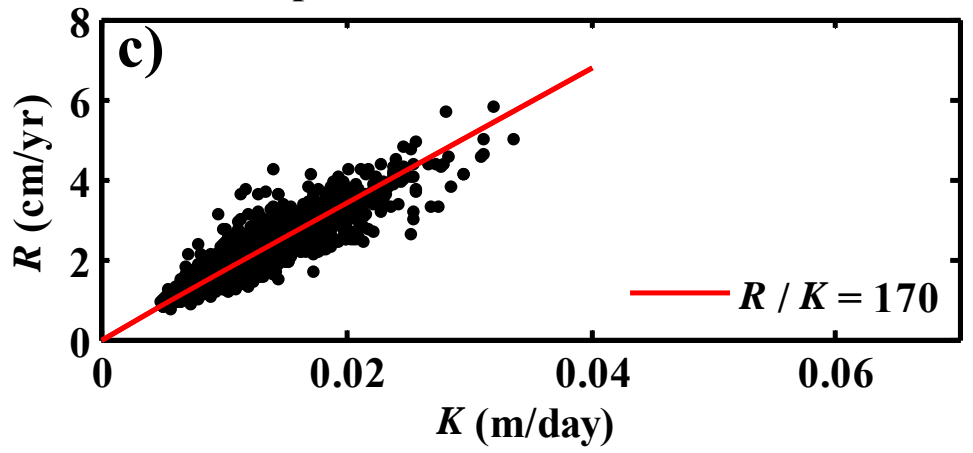

Figure 4.21. Recharge-conductivity pairs (a) as used in the Milrow 2002 model, (b) generated from the MCMC algorithm, and (c) extracted from the MCMC results for use in the revised model.

\subsubsection{Long Shot}

Figure 4.22 exhibits the conditioning results for Long Shot. The first data set, $D_{1}$, refers to the combined MT-converted concentrations and salinity data from EH-5, and the second data set, $D_{2}$, refers to the head data obtained from a number of wells that are closely spaced such that they all exist within one model cell. Compared to Milrow, the prior distribution for $R$ was shifted to higher values and that for $K$ was shifted to lower values. This 
was done in the 2002 model to account for the seemingly deeper transition zone at Long Shot as per the salinity data at EH-5. This resulted in a prior distribution for the rechargeconductivity ratio having its peak density associated with a value of $R / K$ of about 240 as compared to a value of about 79 for the Milrow $R / K$ prior.

Conditioning on the first data set yields a shift toward higher recharge values compared to the prior (Figure 4.22a). The peak of the posterior distribution is slightly higher than the peak of the prior and the spreading expressed by $\sigma_{R}$ is smaller. After conditioning on the head data, a shift toward lower recharge values occurs, accompanied by a reduction in the spreading of the density function and an increase in the peak. The peak of the prior distribution is located around an $R$ value of $2.2 \mathrm{~cm} / \mathrm{yr}$ and the peak of the final marginal posterior for $R$ is located at a value of $R$ of about $2.6 \mathrm{~cm} / \mathrm{yr}$.

For hydraulic conductivity data, conditioning does not shift the curve, but reduces the spreading of the density function and increases its peak. The first conditioning on $D_{1}$ yields an increase in the peak density of about 50 percent (compare the blue and red lines in Figure $4.22 \mathrm{~b}$ ). The peak location of the posterior is located at a slightly higher $K$ value than that of the prior. When conditioning on the heads using the first posterior (the red line) as a prior, the mode of the distribution (i.e., the peak location) is shifted from a value of about 0.008 to a higher $K$ value of about $0.01 \mathrm{~m} / \mathrm{d}$. The spreading of the density function is slightly reduced after conditioning on heads. The peak of the final marginal posterior of $K$ (green line) has increased about 60 percent compared to the prior. The reduction of the $K$ range is very significant where the prior distribution covered a range of about $[0.002$ to $0.035 \mathrm{~m} / \mathrm{d}$ ], whereas the final posterior's range is about $[0.004$ to $0.022 \mathrm{~m} / \mathrm{d}]$.

The MCMC results for the recharge-conductivity ratio at Long Shot show that the first conditioning on $D_{1}$ significantly moves the density curve toward higher $R / K$ values (Figure 4.22c). This means that the combined concentration data set implies a deeper transition zone than implemented in the 2002 model through the prior distribution. Not only has the distribution moved toward higher ratios, its spreading has also decreased and the peak has increased 50 percent or more compared to the prior. The location of the peak has also moved from a value of about 240 to a value of about 340 after the first conditioning. Subsequent conditioning on the heads produces an opposite movement toward lower ratios with a decreased spreading, as is evident from the values of the standard deviation $\sigma_{R / K}$ for the two distributions. The peak value of the final posterior is about 50 percent higher than the peak of the first posterior and is more than doubled compared to the peak of the prior. The mode of the $R / K$ final posterior is at a value of $R / K$ of about 255 as opposed to 340 in the first posterior.

It is interesting that the impact of the head data in Long Shot is stronger than the impact of the Milrow head data. This may be attributed to the fact that the head data at Long Shot are more consistent with the salinity data from EH-5 (i.e., both suggesting similar transition zone locations), whereas at Milrow the head and salinity data at UAe-2 suggest different transition zone locations. In both sites, the head data seem to suggest a shallower transition zone than the combined concentration data do. This is demonstrated by the shift toward lower $R / K$ values caused by conditioning on the heads. It is also interesting that at both sites, the head data helped in tightening the distribution of the $R / K$ posterior $\left(\sigma_{R / K}\right.$ decreased from 30.4 to 24.4 at Milrow and from 61.3 to 39.3 at Long Shot after conditioning on heads). 

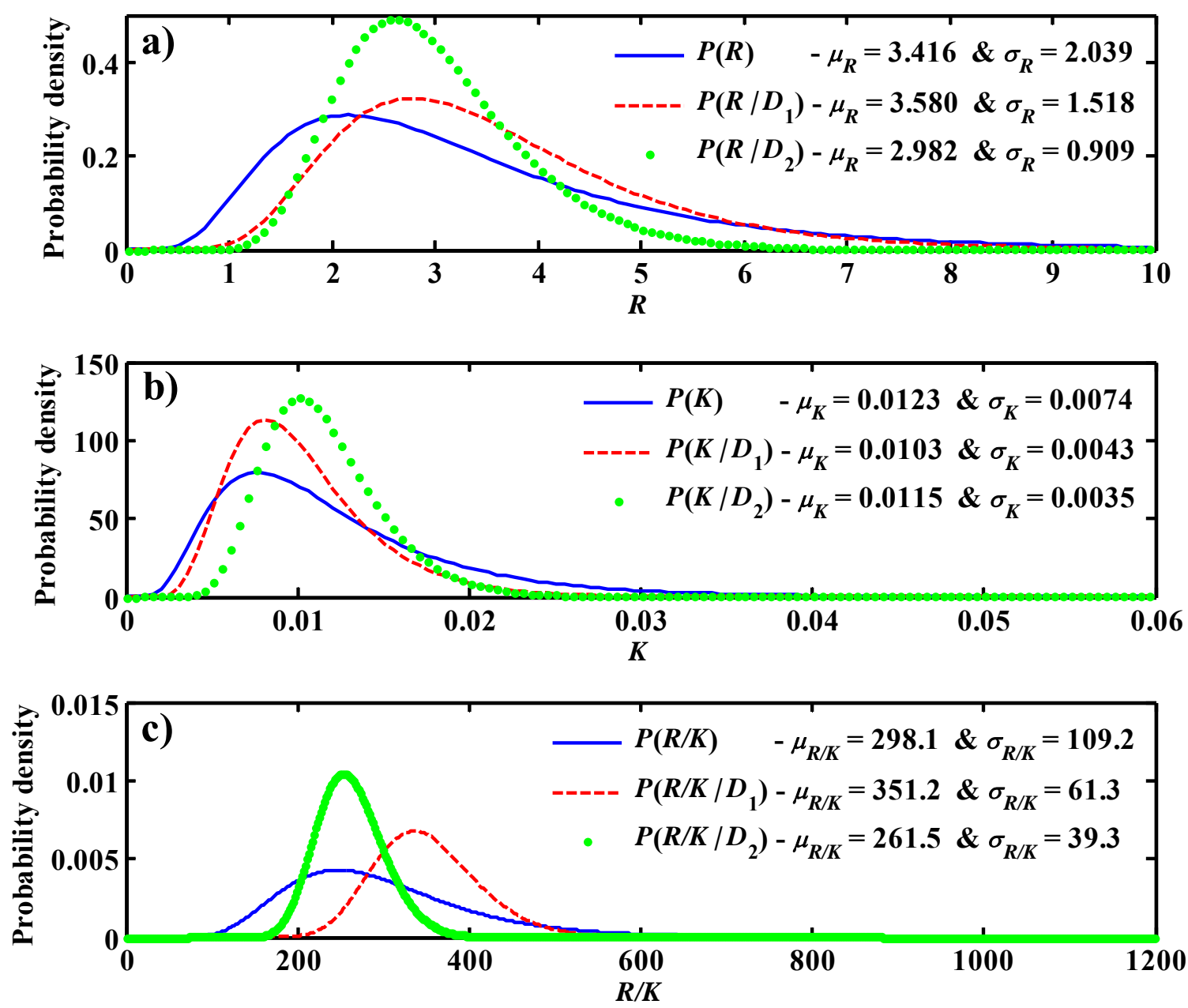

Figure 4.22. Results of the sequential conditioning approach for Long Shot where $D_{1}$ is the concentration data set (salinity at EH-5 and MT-converted concentrations) and $D_{2}$ is the head data at a number of wells.

The above results for Long Shot are based on the theoretical prior and posterior fitted to the original 240 realizations and the MCMC-generated 90,000 values (100,000 iterations minus the 10,000 burn-in iterations), respectively. It is useful to compare the actual values of $R$ and $K$ for three cases as was done in the Milrow case. Figure 4.23 displays (a) the $240 R-K$ pairs of the 2002 model, (b) the 90,000 pairs generated by MCMC, and (c) the 2,000 pairs (used in flow and transport modeling at Long Shot) selected at random out of the 90,000 pairs. The plots also have the line of equal $R / K$ plotted on the data for the $R / K$ value with the highest prior or posterior density (i.e., the mode of the prior or posterior distribution). 
$240 R$ - $K$ pairs used in the 2002 model

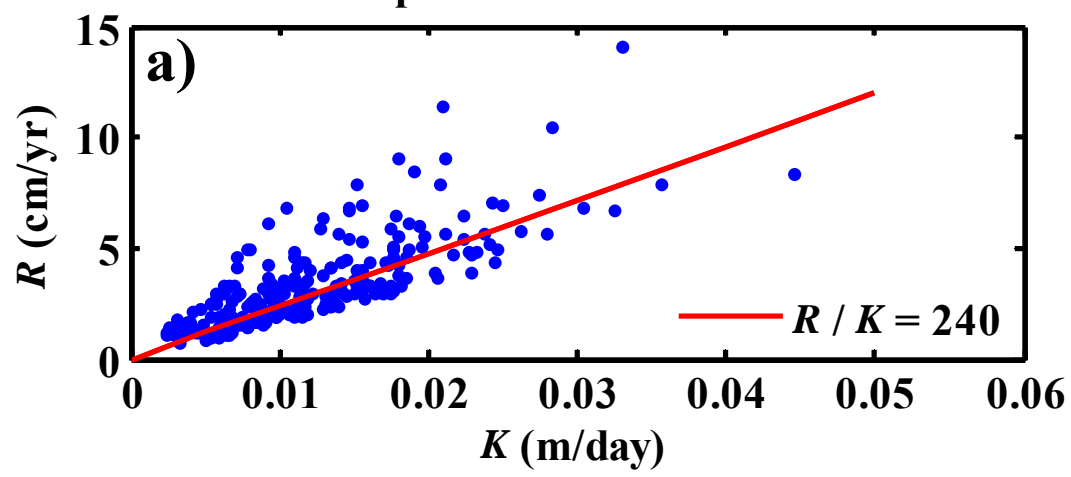

$90,000 R$ - K pairs generated by MCMC

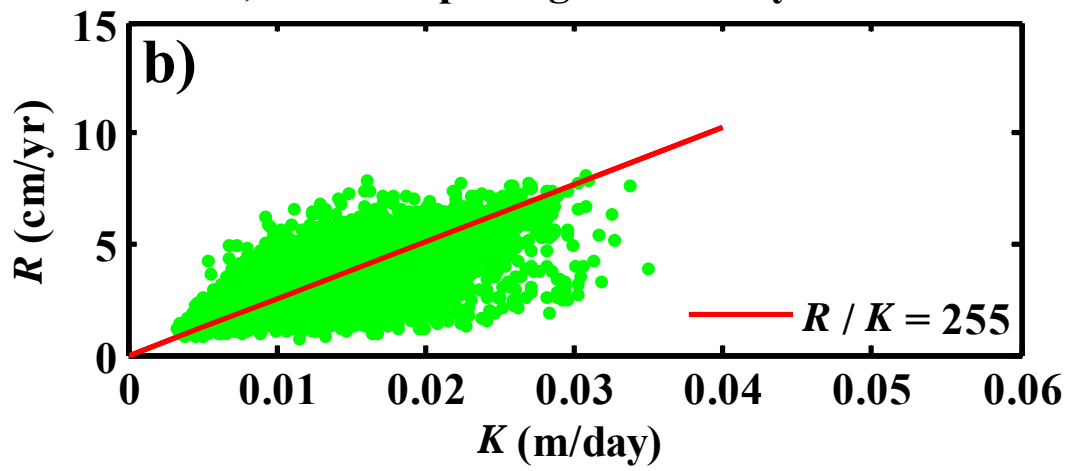

$2000 R$ - $K$ pairs to be used in the revised model

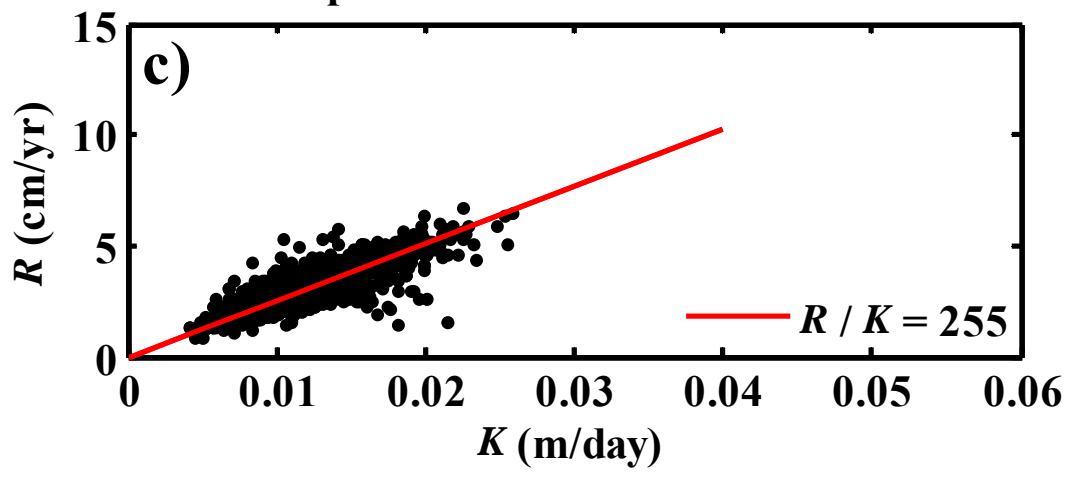

Figure 4.23. Recharge-conductivity pairs (a) as used in the Long Shot 2002 model, (b) generated from the MCMC algorithm, and (c) extracted from the MCMC results for use in the revised model.

The posterior pairs show a very strong positive correlation, which was not as strong in the prior pairs. The range of $R / K$ is very tight around the 255 value, whereas in the prior, the range had a long tail toward the higher $R / K$ values of Figure $4.23 \mathrm{a}$. The prior data had conductivity values reaching $0.045 \mathrm{~m} / \mathrm{d}$, whereas the pairs in Figure $4.23 \mathrm{~b}$ and c have a maximum conductivity of about 0.035 and $0.027 \mathrm{~m} / \mathrm{d}$, respectively. Similarly, the prior data have a maximum recharge value of about $15 \mathrm{~cm} / \mathrm{yr}$, whereas the pairs in Figure $4.23 \mathrm{~b}$ and c have a maximum recharge of about 8.1 and $6.7 \mathrm{~cm} / \mathrm{yr}$, respectively. 


\subsubsection{Cannikin}

Compared to Milrow and Long Shot, Cannikin has a recharge prior distribution with a higher mean (3.614 compared to 3.416 at Long Shot and 2.083 at Milrow) and larger spreading (Figure 4.24) where the standard deviation of the prior is 2.247 compared to 2.039 at Long Shot and 1.379 at Milrow. The conductivity prior is very similar to that at Long Shot. This results in an $R / K$ prior with a higher mean (335.3 compared to 298.1 at Long Shot and 145.5 at Milrow) and higher $\sigma_{R / K}(161.3$ compared to 109.2 at Long Shot and 103.5 at Milrow).
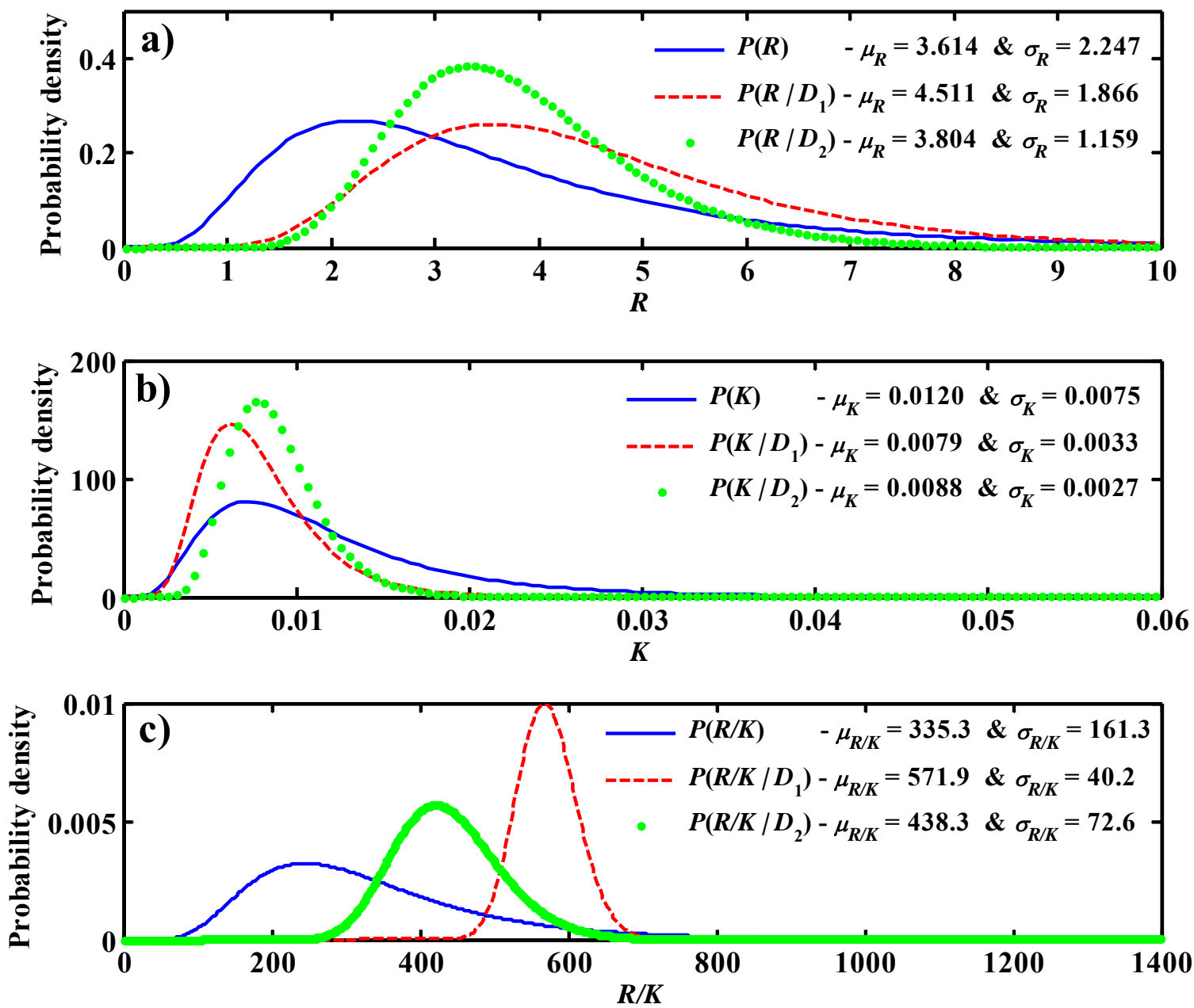

Figure 4.24. Results of the sequential conditioning approach for Cannikin, where $D_{1}$ is the concentration data set (salinity at UAe-1 and MT-converted concentrations) and $D_{2}$ is the head data at UAe-1, UA-1, and HTH-1.

Conditioning on the combined concentration data for recharge yields a posterior with higher recharge values, where the mean increased from 3.614 to 4.511 and less spreading, and where the standard deviation decreased from 2.247 to 1.866. Conditioning on the heads in the sequential conditioning approach shifts the posterior toward lower recharge values and further decreases the spreading. Accordingly, the density peak has increased after the second 
conditioning on the heads. The mode of the distribution increased from a value of 2.1 in the prior to a value of about 3.5 after the first conditioning on the combined concentration data and then subsequently decreased to a value of about 3.25 after conditioning on the heads.

For $K$, the first conditioning yields a dramatic reduction in spreading, where the high$K$ tail of the prior distribution is significantly trimmed. This yields a first posterior with a higher density than the prior and with a peak density that is almost double the prior peak density. The location of the peak (i.e., the mode of the distribution) has slightly shifted to a lower $K$ value. The second conditioning on heads results in a final $K$ posterior that has a slightly higher peak density than the first posterior and is also slightly shifted toward higher $K$ values. The spreading on the distribution has further decreased after the second conditioning. The mode of the final posterior is slightly higher than the mode of the prior and is also higher than the mode of the first posterior.

The recharge-conductivity ratio is impacted the most by conditioning. Figure $4.24 \mathrm{c}$ shows that when conditioning on the combined concentration data set, a very dramatic increase in the ratio occurs, where the distribution has almost totally shifted to higher $R / K$ values. $\mu_{R / K}$ has increased from 335.3 to 571.9 and the spreading has dramatically decreased from 161.3 to 40.2 . This resulted in about a threefold increase in the peak density and the mode of the distribution shifted from an $R / K$ value of about 240 to a value of about 570 . Subsequent conditioning on heads introduces more uncertainty into the distribution of $R / K$, where $\sigma_{R / K}$ has increased from 40.2 to 72.6 . The peak density has decreased about 40 percent and the mode of the distribution moved to a value of $R / K$ of about 420 .

The head data at Cannikin are available from three wells, UAe-1, UA-1, and HTH-1. This data set has significant variability and is also relatively larger in size than other head data sets. Therefore, in its attempts to honor the head data through the second conditioning, the $\mathrm{MCMC}$ algorithm results in a wider $R / K$ distribution with the shallow transition zone realizations (matching the heads and having lower $R / K$ values) contributing to the posterior $R / K$ distribution.

The above results for Cannikin are based on the theoretical prior and posterior fitted to the original 260 realizations and the MCMC-generated 90,000 values $(100,000$ iterations minus the 10,000 burn-in iterations), respectively. It is useful to compare the recharge and conductivity values for three cases (Figure 4.25). The figure shows (a) the $260 R-K$ pairs of the 2002 model, (b) the 90,000 pairs generated by MCMC, and (c) the 2,000 pairs (used in flow and transport modeling at Cannikin) selected at random out of the 90,000 pairs. The plots also have the line of equal $R / K$ plotted on the data for the $R / K$ value with the highest prior or posterior density (i.e., the mode of the prior or posterior distribution).

The posterior pairs show a strong positive correlation, which was not as strong in the prior pairs. Unlike the previous two sites, the range of $R / K$ in the posterior (Figure $4.25 \mathrm{~b}$ ) is not very tight around the 420 value. There is some scatter at lower $R / K$ values, but they do not have high density. Also, the prior data had conductivity values reaching $0.045 \mathrm{~m} / \mathrm{d}$, whereas the 2,000 pairs to be used in the revised model have a maximum conductivity of about $0.025 \mathrm{~m} / \mathrm{d}$. The maximum recharge value in the prior data is about $18.9 \mathrm{~cm} / \mathrm{yr}$, and the maximum recharge values in the 90,000 pairs and the 2,000 pairs that are used in the current model are about 11.5 and $10 \mathrm{~cm} / \mathrm{yr}$, respectively. 


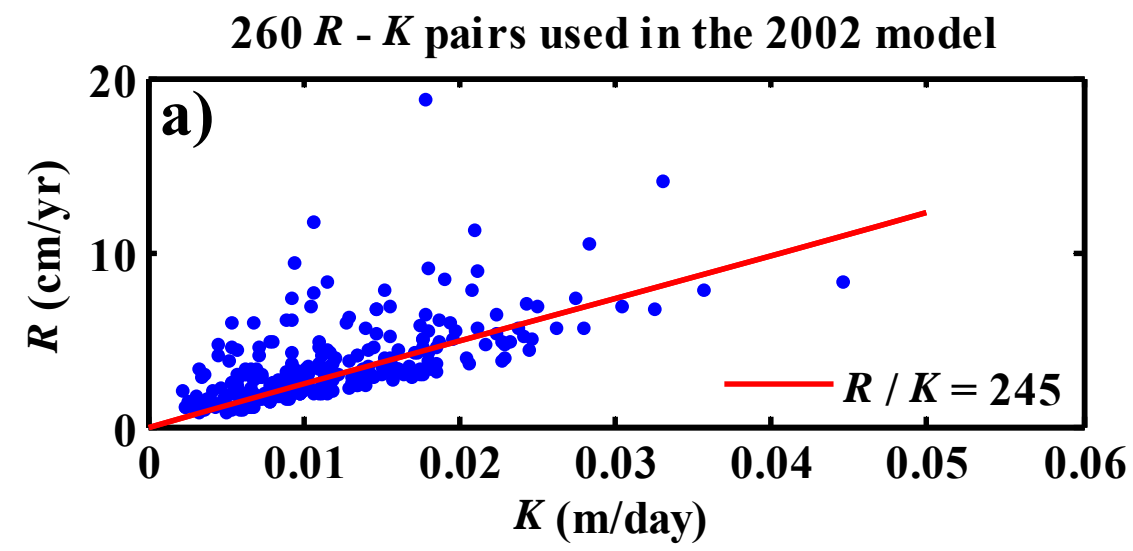

$90,000 R$ - $K$ pairs generated by MCMC

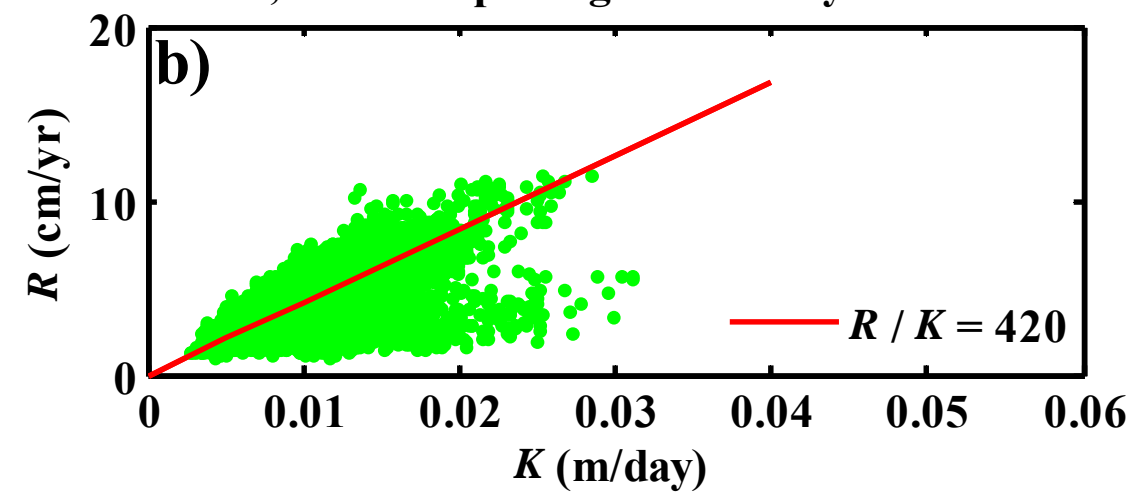

$2000 R$ - $K$ pairs to be used in the revised model

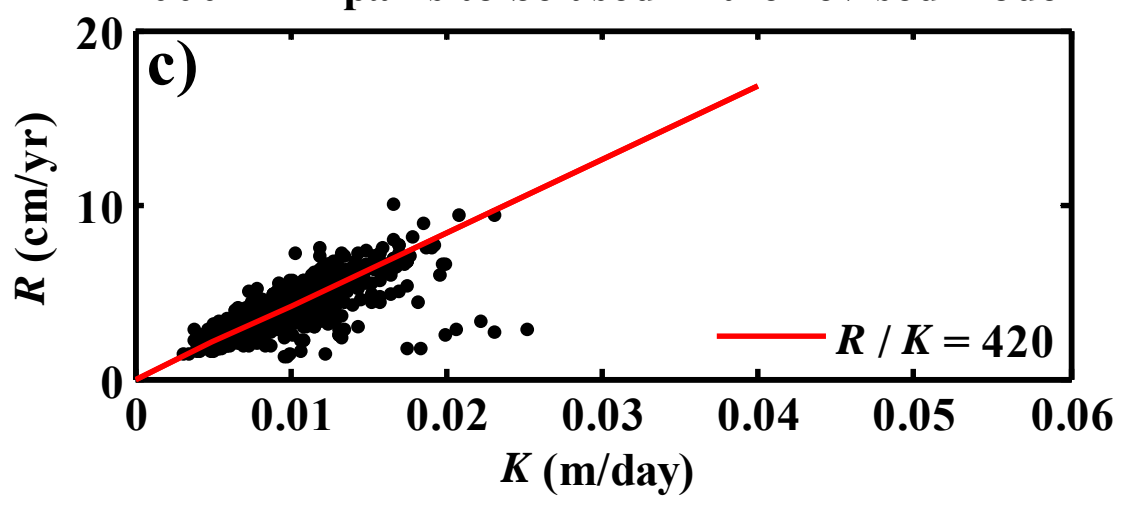

Figure 4.25. Recharge-conductivity pairs (a) as used in the Cannikin 2002 model, (b) generated from the MCMC algorithm, and (c) extracted from the MCMC results for use in the revised model.

\subsubsection{Summary of MCMC Results}

The combined concentration data set impacts the recharge distribution for the three sites, with Long Shot recharge distribution impacted the least. In all three sites, this combined concentration data set yields a posterior with higher recharge values than the prior (i.e., shifts the distribution to the right side of the figure) and as such, the mean of the posterior is higher than the prior mean. In all three sites, the spreading of the distribution (i.e., $\sigma_{R}$ ) decreases after conditioning on the concentration data set (Table 4.5). 
The impact of the combined concentration data set $\left(D_{1}\right)$ on the hydraulic conductivity distribution is strong for all three sites. The impact of this conditioning on the mean $\mu_{K}$ is not significant, but the impact on $\sigma_{K}$ is more pronounced. The location of the distribution does not shift, but the tails are significantly trimmed, leading to a significant reduction of uncertainty represented by $\sigma_{K}$ (Table 4.5).

For $R / K$, the first conditioning yields dramatic effects in all three sites. In all three sites, the distribution shifts toward higher $R / K$ values and the spreading dramatically decreases (about 70 percent reduction in $\sigma_{R / K}$ at Milrow, 44 percent reduction at Long Shot, and about 75 percent reduction at Cannikin). The shift toward higher ratios causes an increase in the distribution mean of about 39 percent at Milrow, 18 percent at Long Shot, and about 70 percent at Cannikin. The dramatic impact at Cannikin is due to the fact that the salinity data at UAe- 1 and the MT data consistently show a deeper transition zone (thus requiring higher $R / K$ values) than that suggested by the prior distribution in the 2002 model.

The impact of heads through the second conditioning (using the first posterior as a prior) is smaller than that of the combined concentration data, mainly due to the limited size of head data at all sites relative to the MT data. However, they still have an impact on the final posterior. The heads shift the recharge distribution toward lower values. This shift is smallest at Milrow and largest at Long Shot and Cannikin. This shift is accompanied by a reduction in spreading (compared to first posterior) that is more or less equivalent at all three sites.

Conditioning on heads led to a shift toward higher hydraulic conductivities at the three sites. These shifts were very similar in the three sites. The standard deviation of the density function decreased at all three sites in a similar manner.

The conditioning impact on the ratio $R / K$ is more significant than on the individual parameters. In all sites, the final $R / K$ posterior shifts toward lower values of $R / K$ than found in the first posterior. Compared to the prior, the final posterior leads to higher ratios, except at Long Shot where it indicates similar values with a smaller spread. In all three sites, significant uncertainty reduction (reduced $\sigma_{R / K}$ ) results from the MCMC conditioning on the different data sets.

Relative to the original prior, the final posterior that will be used for modeling has a dramatic reduction in the variance indicating that the MCMC conditioning constrained the parameter distributions to honor field data. The largest reduction in $R / K$ uncertainty was achieved at Milrow and the smallest reduction occurred at Cannikin (Table 4.5). Overall, equivalent uncertainty reductions occurred at all sites. 
Table 4.5. Summary of MCMC results showing percent reduction in variance.

\begin{tabular}{|c|c|c|c|c|c|c|c|}
\hline \multirow[t]{2}{*}{ Site } & \multirow[t]{2}{*}{ Conditioning on } & \multicolumn{3}{|c|}{$\begin{array}{c}\% \text { Reduction in Variance } \\
\left(\text { Prior } \sigma^{2} \text {-Posterior } \sigma^{2}\right) / \text { Prior } \sigma^{2}\end{array}$} & \multicolumn{3}{|c|}{$\begin{array}{c}\% \text { Reduction in Variance } \\
\left(\text { Prior } \sigma^{2} \text {-Final Posterior } \sigma^{2}\right) / \text { Prior } \sigma^{2}\end{array}$} \\
\hline & & $R$ & $K$ & $R / K$ & $R$ & $K$ & $R / K$ \\
\hline \multirow{2}{*}{ Milrow } & $\begin{array}{l}D_{1} \text { (Combined UAe- } 2 \text { salinity data and } \\
\text { MT-converted concentration) }\end{array}$ & 35.56 & 72.03 & 91.37 & \multirow{2}{*}{74.01} & \multirow{2}{*}{96.15} & \multirow{2}{*}{94.44} \\
\hline & $D_{2}$ (UAe-2 head data and other wells) & 59.67 & 44.43 & 35.58 & & & \\
\hline \multirow{2}{*}{$\begin{array}{l}\text { Long } \\
\text { Shot }\end{array}$} & $\begin{array}{l}D_{1} \text { (Combined EH- } 5 \text { salinity data and } \\
\text { MT-converted concentration) }\end{array}$ & 44.57 & 67.78 & 68.49 & \multirow{2}{*}{80.13} & \multirow{2}{*}{77.63} & \multirow{2}{*}{87.05} \\
\hline & $D_{2}$ (EH-5 head data and other wells) & 64.14 & 30.56 & 58.90 & & & \\
\hline \multirow{2}{*}{ Cannikin } & $\begin{array}{l}D_{1} \text { (Combined UAe- } 1 \text { salinity data and } \\
\text { Mt-converted concentration) }\end{array}$ & 31.04 & 80.64 & 93.79 & \multirow{2}{*}{73.40} & \multirow{2}{*}{87.98} & \multirow{2}{*}{79.74} \\
\hline & $D_{2}$ (UAe-1, UA-1, and HTH-1 head data) & 61.42 & 33.06 & $-226.15^{*}$ & & & \\
\hline
\end{tabular}

${ }^{*}$ Negative value indicates an increase in variance

\section{REVISED FLOW MODELING}

For the updated flow modeling for the sites, the same model domain, meshes, and boundary conditions as used in the 2002 model are applied. The changes to the model are:

1) The recharge and hydraulic conductivity are generated from the final posterior distributions presented in the previous section.

2) The depth-decay of the fracture porosity is based on the profiles (Figure 4.5) provided by Unsworth et al. (2005). Details on populating the simulation domain with porosity values are presented next in Section 5.1. The original 2002 model had a uniform fracture porosity in the domain that was uncertain (i.e., drawn from a distribution for each realization) as described earlier in the data description section.

3) The cavity and rubble chimney are assumed to have a porosity value of 0.4 for all realizations at the three sites, as compared to a value of 0.07 in the original model.

4) The number of realizations for all sites is 2,000, whereas in the Hassan et al. (2002) model the number varied between 240 and 300 realizations.

All other parameters including the anisotropy ratio, local dispersivity, macrodispersivity, glass dissolution coefficient, and matrix diffusion remain the same as in the 2002 model. It is important to recall that porosity is needed in the density driven flow model, which involves simultaneous solution of the groundwater flow equation and the advection-dispersion equation for seawater intrusion.

The anisotropy ratio is 1:10 in the entire domain except in the cavity and chimney area, where it is assumed that the hydrologic impact of the test and the formation of the chimney enhances vertical conductivity. As a result, no anisotropy is applied in those model cells, resulting in a vertical hydraulic conductivity value 10 times greater than the rest of the domain. Figure 5.1 shows the upper left portion of the simulation domain for the three tests with the chimney location highlighted. 

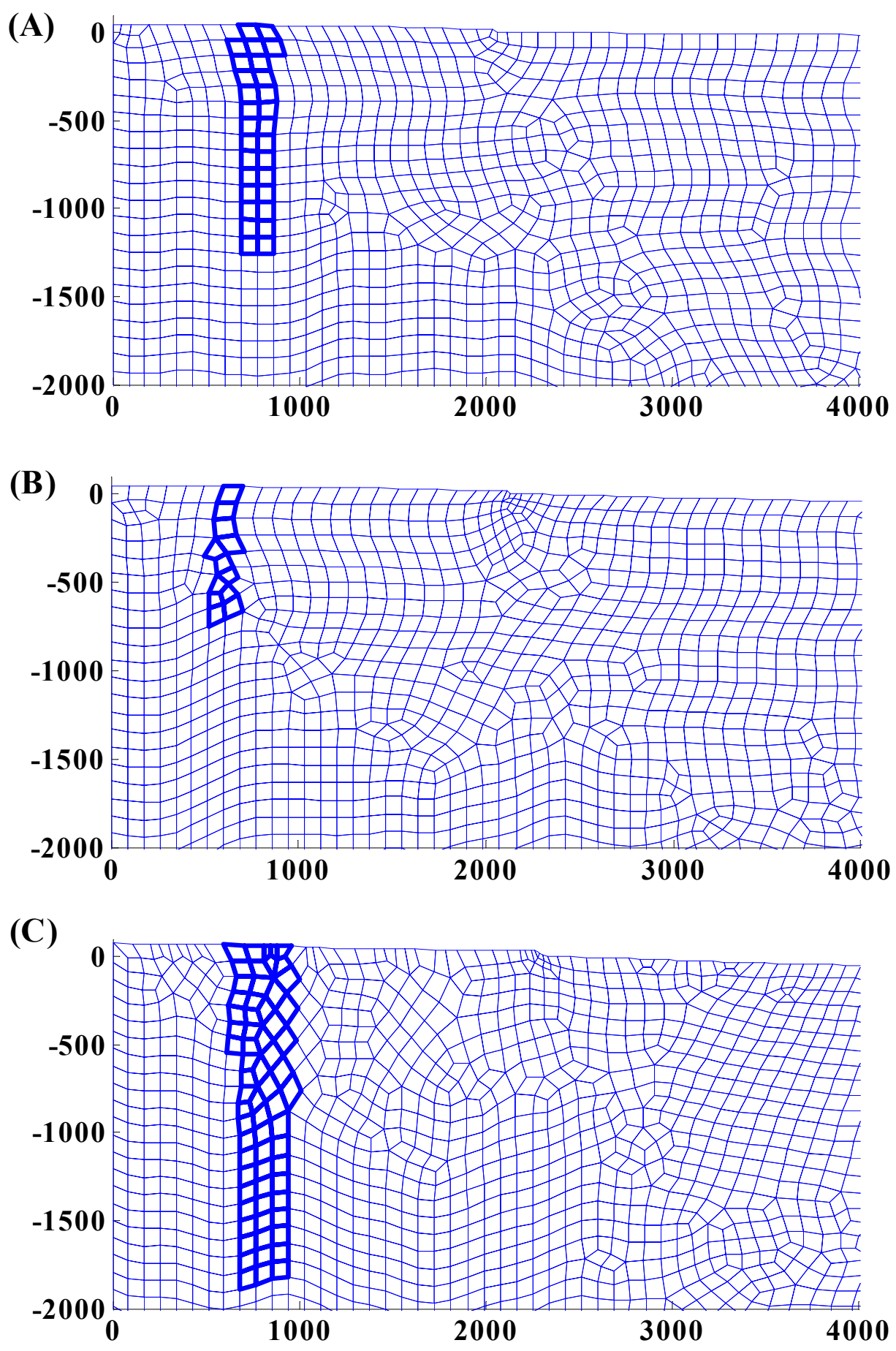

Figure 5.1. Expanded view of the upper left portion of each model domain, showing the nuclear chimney included in the flow and transport simulations for Milrow (A), Long Shot (B), and Cannikin $(\mathrm{C})$. 
The MCMC analysis presented in Section 4 resulted in a sample of size 90,000 values for the recharge and conductivity that are drawn from the posterior distribution. Two thousand pairs are selected from this sample using a stratified sampling technique. The 90,000 pairs are divided into 2,000 segments or layers with 45 pairs in each layer. A random number is then drawn for each layer from a uniform $[0,1]$ distribution. These random numbers are then scaled up to provide a set of random numbers uniformly distributed in the range [1,45]. Thus, a sample of $R-K$ is selected at random from each layer using these uniform random numbers resulting in 2,000 pairs of $R$ and $K$. These pairs are shown in Figures 4.21, 4.23, and 4.25 for Milrow, Long Shot, and Cannikin, respectively.

Fracture porosity is generated from the profiles (Figure 4.5) of Unsworth et al. (2005). These profiles provide a lower bound, an upper bound, and an intermediate profile depending on the value of an exponent $m$ used in Archie's law (Unsworth et al., 2005). For each realization, samples are taken randomly and uniformly between the upper and lower bounds. The two bounds start at the top from almost the same value and then diverge with depth (Figure 4.5). A random number is drawn from a uniform distribution $[0,1]$ and a linear interpolation is performed between the two bounds at each depth. For example, if the random number is 0.5 , then the porosity value is exactly halfway between the two bounds at all depths. In the lateral direction of the model domain ( $x$-direction), porosity is constant at the same depth across the domain.

The FEFLOW code (Diersch, 1998) is used for the flow simulations of the revised model. FEFLOW is a finite-element simulation package available from the WASY Institute for Water Resources Planning and Systems Research Ltd. that is developed for twodimensional and three-dimensional density-dependent flow, mass, and heat transport processes in groundwater, and is generally well-suited for the Amchitka problem.

The groundwater flow problem modeled here requires a description of variable density fluid flow coupled with transport of both salt and heat. The fluid flow is simulated using a form of the Darcy equation generalized for a variable-density fluid. The approach is formulated in terms of the equivalent freshwater head, $h_{f}$, and the freshwater hydraulic conductivity, $\mathbf{K}_{\mathbf{f}}$, as

$$
h_{f}=\frac{p}{\rho_{0} g}+z
$$

and

$$
\mathbf{K}_{\mathbf{f}}=\frac{\rho_{0} g}{\mu} \times \mathbf{k}
$$

respectively, where $p$ is fluid pressure, $\rho_{0}$ is a reference density of freshwater, $g$ is gravitational acceleration, $z$ is elevation, $\mu$ is fluid viscosity, and $\mathbf{k}$ is the permeability tensor. $\mathbf{K}_{\mathrm{f}}$ is calculated through Equation (5.2) using actual local concentration and temperature effects on density and viscosity implied by the buoyancy term and the viscosity function, given the appropriate reference temperature and concentration for the $K$ measurements. The flow equation can then be written for Darcy flux as 


$$
\boldsymbol{q}=-\mathbf{K}_{\mathbf{f}} \cdot\left(\nabla h_{f}-\frac{\left(\rho-\rho_{0}\right)}{\rho_{0}} \nabla z\right)
$$

The density-dependent relations in the coupled transport system employ the extended Boussinesq approximation (Diersch, 1998), where the conservation of fluid mass is described in the generalized form

$$
S_{S} \frac{\partial h}{\partial t}+\nabla \cdot \boldsymbol{q}=Q_{p}+Q_{E B}(C, T)
$$

where $S_{S}$ is the specific storage coefficient (compressibility), $Q_{p}$ is the specific source/sink rate of fluid, and $Q_{E B}(C, T)$ is a term of the extended Boussinesq approximation that accounts for temporal changes in concentration and/or temperature and variation in density in directions orthogonal to the direction of flow. The representative equation for mass transport is given as (Diersch, 1998)

$$
R_{d} \frac{\partial C}{\partial t}+\boldsymbol{q} \cdot \nabla C-\nabla \cdot\left(\mathbf{D}_{h} \cdot \nabla C\right)+Q_{p} C=Q_{C}
$$

where $R_{d}$ is the retardation factor, $\mathbf{D}_{h}$ is the tensor of hydrodynamic dispersion, $C$ is the concentration, and $Q_{C}$ is the source/sink function of mass (Diersch, 1998). Table 5.1 lists the parameters used in all FEFLOW simulations presented in this study. It should be noted here that the current study assumes isothermal conditions, and thus the temperature variation aspects mentioned above are not considered here. The heat effects from the nuclear test and the geothermal heat impacts were evaluated in the sensitivity studies in Hassan et al. (2002).

Table 5.1. Parameters used in FEFLOW to solve the density-driven flow problem

\begin{tabular}{lc}
\multicolumn{1}{c}{ Parameter } & Value \\
\hline Freshwater density $\left(\mathrm{kg}_{\text {water }} / \mathrm{m}^{3}\right)$ & 1,000 \\
Saltwater density $\left(\mathrm{kg}_{\text {water }} / \mathrm{m}^{3}\right)$ & 1,025 \\
Freshwater concentration $\left(C / C_{\max }\right)$ & $2.67 \times 10^{-3}$ \\
Saltwater concentration $\left(C / C_{\max }\right)$ & 1 \\
Density ratio & 0.025 \\
Diffusivity of solute in fluids $\left(\mathrm{m}^{2} / \mathrm{d}\right)$ & $8.88 \times 10^{-9}$ \\
Specific storage $\left(S_{s}\right)$ & $1.0 \times 10^{-4}$ \\
\hline
\end{tabular}

The geometry of the simulation domain and the boundary conditions are the same as those in the 2002 model (Figure 5.2). A domain length of $8,000 \mathrm{~m}$ is assumed, which is about four times the island half-width, and a thickness of 4,000 $\mathrm{m}$, more than three times the thickness of the freshwater lens in the vertical direction. This provides the flexibility to change model parameters, and thus the location and width of the transition zone, with no boundary effects influencing the resulting solution. The left-hand boundary is assumed to 
coincide with the groundwater divide at the island centerline, and as such is assumed to be a no-flow boundary. The bottom boundary is also assumed to be a no-flow boundary. The right-hand boundary is a specified pressure and a constant concentration boundary. The top boundary is divided into two segments; a freshwater recharge segment representing the island half-width, and a specified pressure, constant concentration segment representing the seafloor (bathymetric profile). The two-dimensional domain is considered to be anisotropic $\left(K_{x x} \neq K_{z z}\right)$ and homogeneous (no spatial variability). Spatial variability only appears when accounting for the chimney porosity and permeability relative to the surrounding area. The basis of this conceptual model is discussed by Hassan et al. (2002), and several sensitivity analyses regarding the assumptions are also presented.

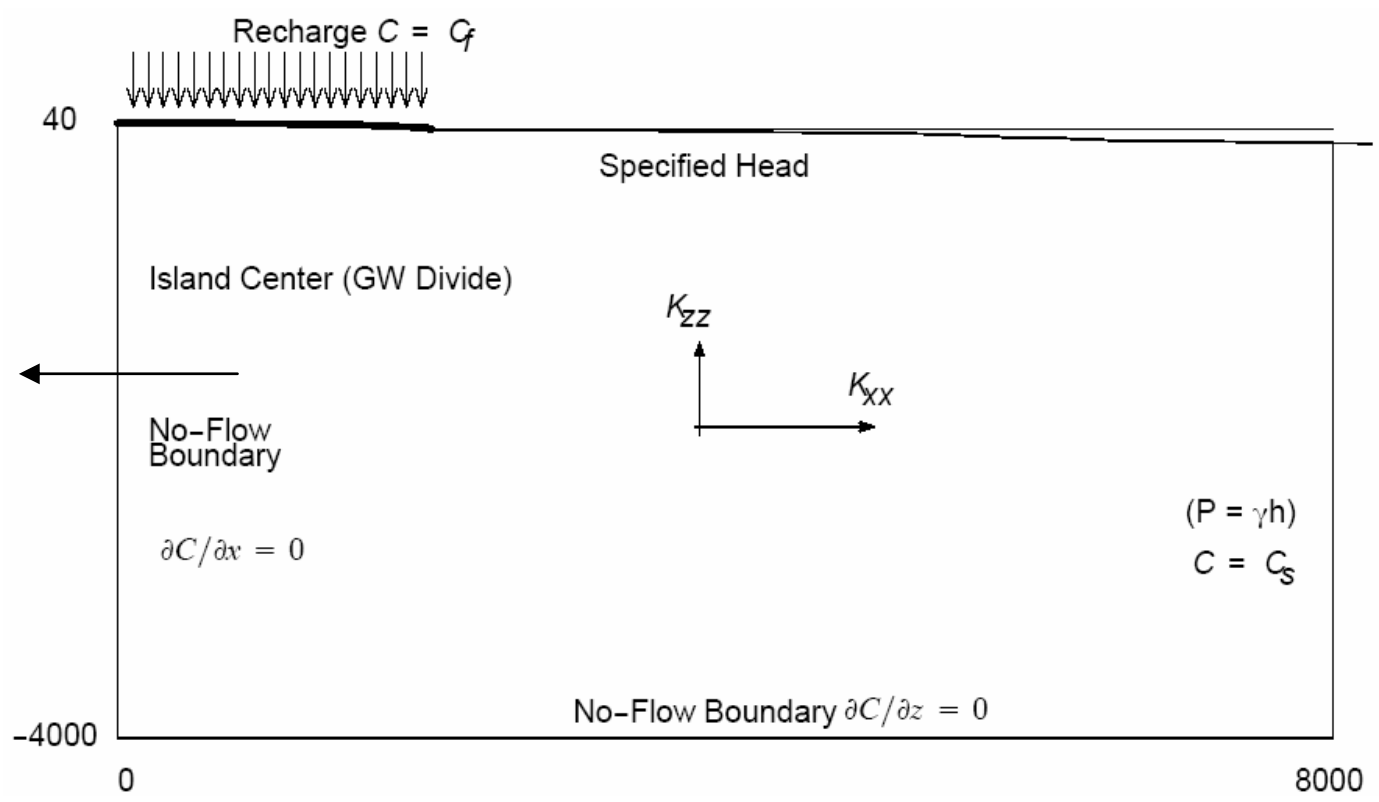

Figure 5.2. Geometry of simulation domain and boundary conditions for the flow problem.

\subsection{Milrow Flow Results}

The 2,000 conductivity-recharge pairs (Figure 4.21c) sampled from the 90,000 MCMC output are used with 2,000 porosity values generated from Unsworth et al. (2005) profiles. The porosity values assigned to each $R-K$ pair is selected at random.

Figure 5.3 compares the generated distributions of recharge, conductivity and porosity to those used in Hassan et al. (2002). The porosity distribution plotted in the figure is at the cavity horizon (i.e., at a domain depth equivalent to the depth to the cavity). All model cells below the cavity have porosity distributions with lower values than the distribution in the figure and all model cells above the cavity have higher porosities. The lowest porosity value in the new distribution (about 0.0083 ) is more than two orders of magnitude higher than the lowest porosity used in the 2002 model, which was about $1.1 \times 10^{-5}$. Also, a large number of realizations (257 out of 300) in Hassan et al. (2002) had porosities less than 0.00083 (i.e., more than an order of magnitude lower than the lowest 
porosity value in the new distribution). The largest porosity value in the distribution used in Hassan et al. (2002), which was about 0.0062, is more than an order of magnitude lower than the largest value in the new distribution, which is about 0.0892 . This will have a strong implication for groundwater velocities and arrival times of radionuclides to the seafloor.
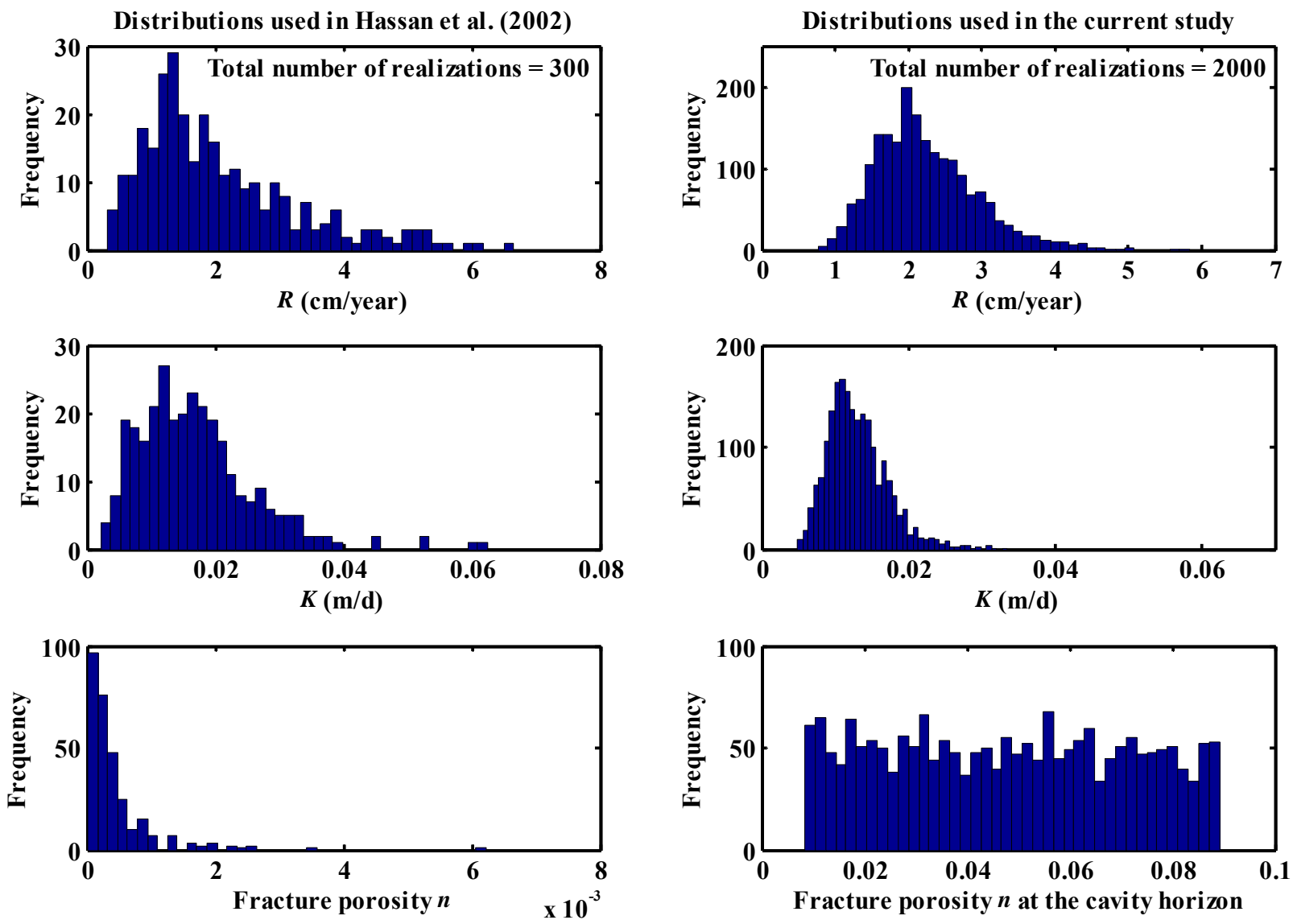

Figure 5.3. Comparison between the distributions of Milrow parameters used in Hassan et al. (2002) and the distributions used in this study based on new data. Fracture porosity distribution in the current model is plotted at the elevation of the test.

The flow and transport parameters used in Milrow simulations are summarized in Table 5.2. The mean and standard deviation shown in the table are slightly different from the values shown for the green posterior in Figure 4.20. This is because the values in Table 5.2 are obtained from the 2,000 generated values but the values in Figure 4.20 are obtained by fitting a lognormal distribution to the sample of 90,000 values generated from the MCMC algorithm. FEFLOW is used to solve the density driven flow problem for the 2,000 realizations with the generated recharge, conductivity, and porosity values (Figure 5.3). Macrodispersivity values are fixed at $100 \mathrm{~m}$ and $10 \mathrm{~m}$ in the longitudinal and transverse directions, respectively. To assess the uncertainty reduction and verify the original 2002 model, the results of these realizations are analyzed at the UAe-2 location. Figure 5.4 displays the simulated concentration and head profiles in UAe-2. The mean of the 2,000 
Table 5.2. Parameter range for Milrow flow and transport simulations.

\begin{tabular}{cccccc}
\hline Parameter & Minimum & Mean & Maximum & $\sigma$ & Distribution \\
\hline$R$ & 0.776 & 2.2384 & 5.8282 & 0.6909 & lognormal \\
$K$ & 0.0049 & 0.013 & 0.0335 & 0.0041 & lognormal \\
$n$ & Figure $4.5 \mathrm{~d}$ lower & Figure $4.5 \mathrm{~d}$ middle & Figure 4.5d upper & $\sim 0.0235$ at cavity & Uniform \\
$A_{L}$ & bound $(m=1)$ & profile $(m=1.5)$ & bound $(m=2)$ & horizon & \\
$A_{T}$ & - & 100 & - & - & - \\
$\alpha_{L}$ & - & 10 & - & - & - \\
$\alpha_{T}$ & - & 5 & - & - & - \\
$k$ & - & 0.5 & - & - & - \\
$k_{g}$ & - & 0.434 & - & - & - \\
\hline
\end{tabular}
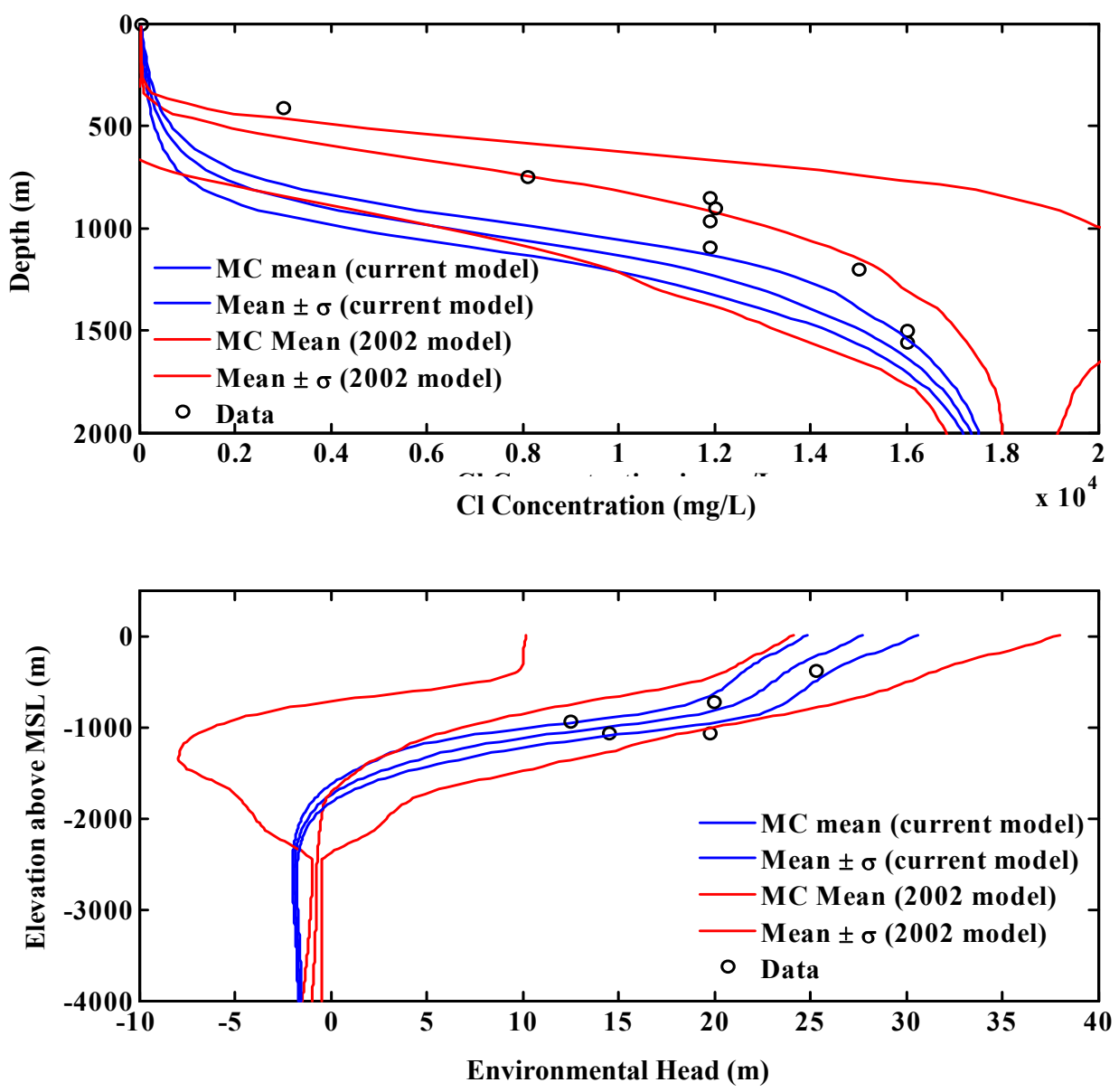

Figure 5.4. Mean \pm one standard deviation for salinity and head profiles at the UAe-2 location at Milrow, as obtained from both the new model and the 2002 model. 
realizations and the mean \pm one standard deviation are plotted. To compare to the original model, Figure 5.4 also shows the results from the 2002 model. A dramatic decrease of uncertainty in the location of the transition zone is achieved by conditioning on the three data sets. The uncertainty bounds, expressed as the mean of the MC realizations $\pm \sigma$, are very tight in the current model compared to the original range of uncertainty (Figure 5.4).

The second important result of the comparison between the current and 2002 uncertainty is that the new results are encompassed within the original model results. That is, the new MC mean and most of the uncertainty bounds are captured within the original range of possible outputs (Figure 5.4). This verifies the original model in the sense that the original model did account for much deeper, as well as shallower, transition zones than what the data from UAe-2 suggested. Also, the match of the MC mean to the head data has improved over the 2002 model, whereas the match to the salinity data is worse. This is expected as the MT data indicated a much deeper transition zone than the water chemistry data at UAe-2 and the heads at UAe- 2 also indicate a deeper transition zone than the salinity data.

\subsection{Long Shot Flow Results}

Similar to Milrow, the recharge and conductivity values for Long Shot are generated from the MCMC results of 90,000 samples (Figure 4.23). The porosity profiles are generated from the bounds given by Unsworth et al. (2005) and shown in Figure 4.5. Figure 5.5 compares the generated distributions of recharge, conductivity, and porosity to those used in Hassan et al. (2002). The porosity distribution plotted in the figure is at the cavity horizon. All model cells below the cavity have porosity distributions with lower values than the one in Figure 5.5 and all model cells above the cavity have higher porosities. The lowest porosity value in the new distribution (about 0.0161 ) is more than three orders of magnitude higher than the lowest porosity used in the 2002 model, which was about $1.0 \times 10^{-5}$. Also, almost all realizations (234 out of 240) in Hassan et al. (2002) had porosities less than 0.00161 (i.e., more than an order of magnitude lower than the lowest porosity value in the new distribution). About 85 realizations ( $\sim 35$ percent) had porosities less than 0.000161 (i.e., two orders of magnitude lower than the minimum value in the new distribution). The largest porosity value in the distribution used in Hassan et al. (2002), which was about 0.0052 , is more than an order of magnitude lower than the largest value in the new distribution, which is about 0.088 .

The flow and transport parameters used in Long Shot simulations are summarized in Table 5.3. FEFLOW is used to solve the density driven flow problem for the 2,000 realizations with the generated recharge, conductivity, and porosity values (Figure 5.5). Similar to Milrow, macrodispersivity values are fixed at $100 \mathrm{~m}$ and $10 \mathrm{~m}$ in the longitudinal and transverse directions, respectively. To assess the uncertainty reduction and verify the original 2002 model, the results of these realizations are analyzed at the EH-5 location for salinity and at the model cell location, where a number of wells are clustered together for heads (Figure 5.6A dramatic decrease of uncertainty in the location of the transition zone is achieved by conditioning on the three data sets. The uncertainty bounds, expressed as the mean of the $\mathrm{MC}$ realizations $\pm \sigma$, are very tight in the current model compared to the original range of uncertainty. 

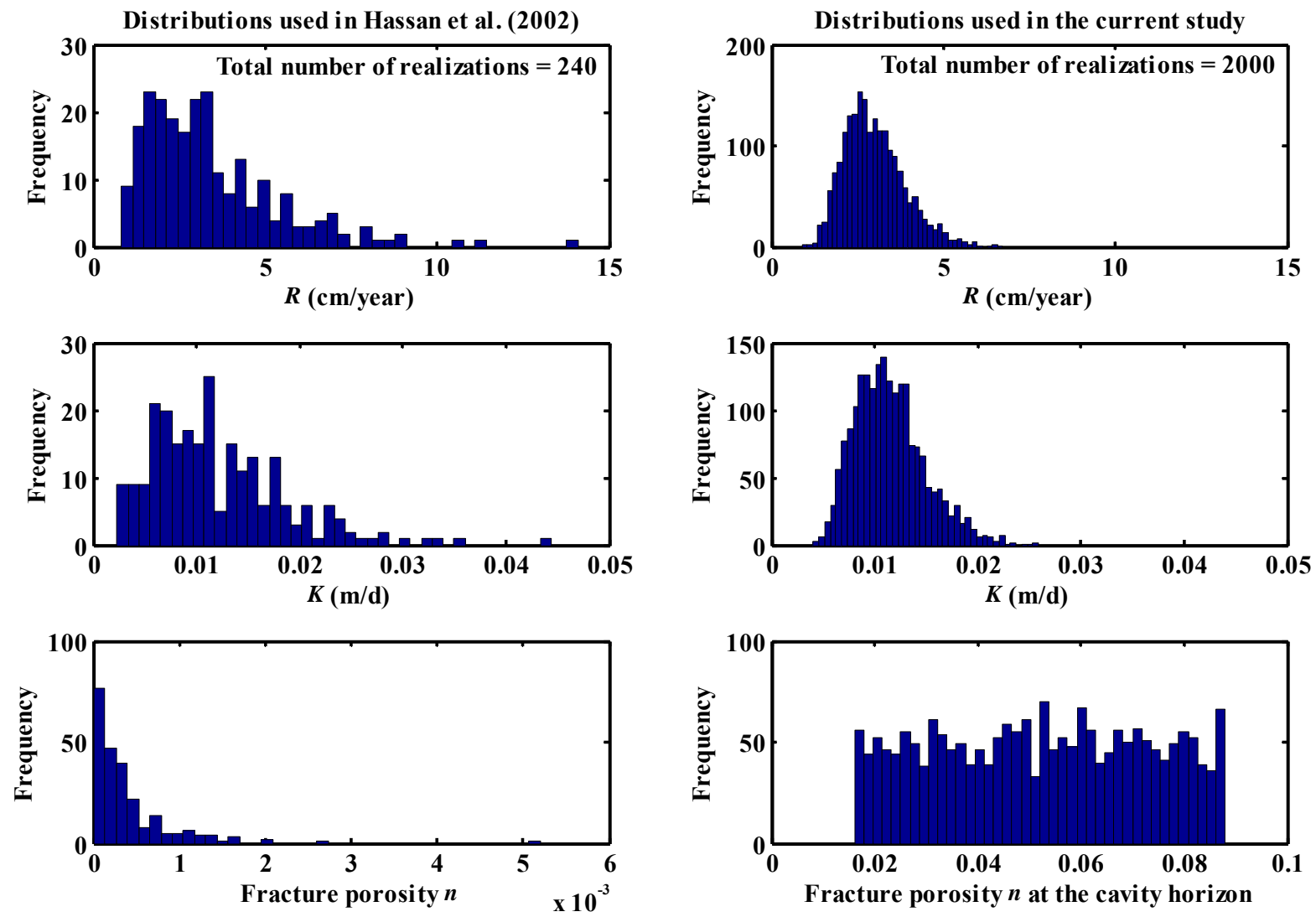

Figure 5.5. Comparison between the distributions of Long Shot parameters used in Hassan et al. (2002) and the distributions used in this study based on new data. Fracture porosity distribution in the current model is plotted at the elevation of the test.

Table 5.3. Parameter range for Long Shot flow and transport simulations.

\begin{tabular}{cccccc}
\hline Parameter & Minimum & Mean & Maximum & $\sigma$ & Distribution \\
\hline$R$ & 0.9006 & 2.986 & 6.7162 & 0.8815 & lognormal \\
$K$ & 0.0041 & 0.0115 & 0.0259 & 0.0034 & lognormal \\
$n$ & Figure $4.5 \mathrm{e}$ lower & Figure $4.5 \mathrm{e}$ middle & Figure $4.5 \mathrm{e}$ upper & $\sim 0.0206$ at cavity & Uniform \\
$A_{L}$ & bound $(m=1)$ & profile $(m=1.5)$ & bound $(m=2)$ & horizon & - \\
$A_{T}$ & - & 100 & - & - & - \\
$\alpha_{L}$ & - & 10 & - & - & - \\
$\alpha_{T}$ & - & 5 & - & - & - \\
$k$ & - & 0.5 & - & - & - \\
$k_{g}$ & - & 0.434 & - & - & - \\
\hline
\end{tabular}



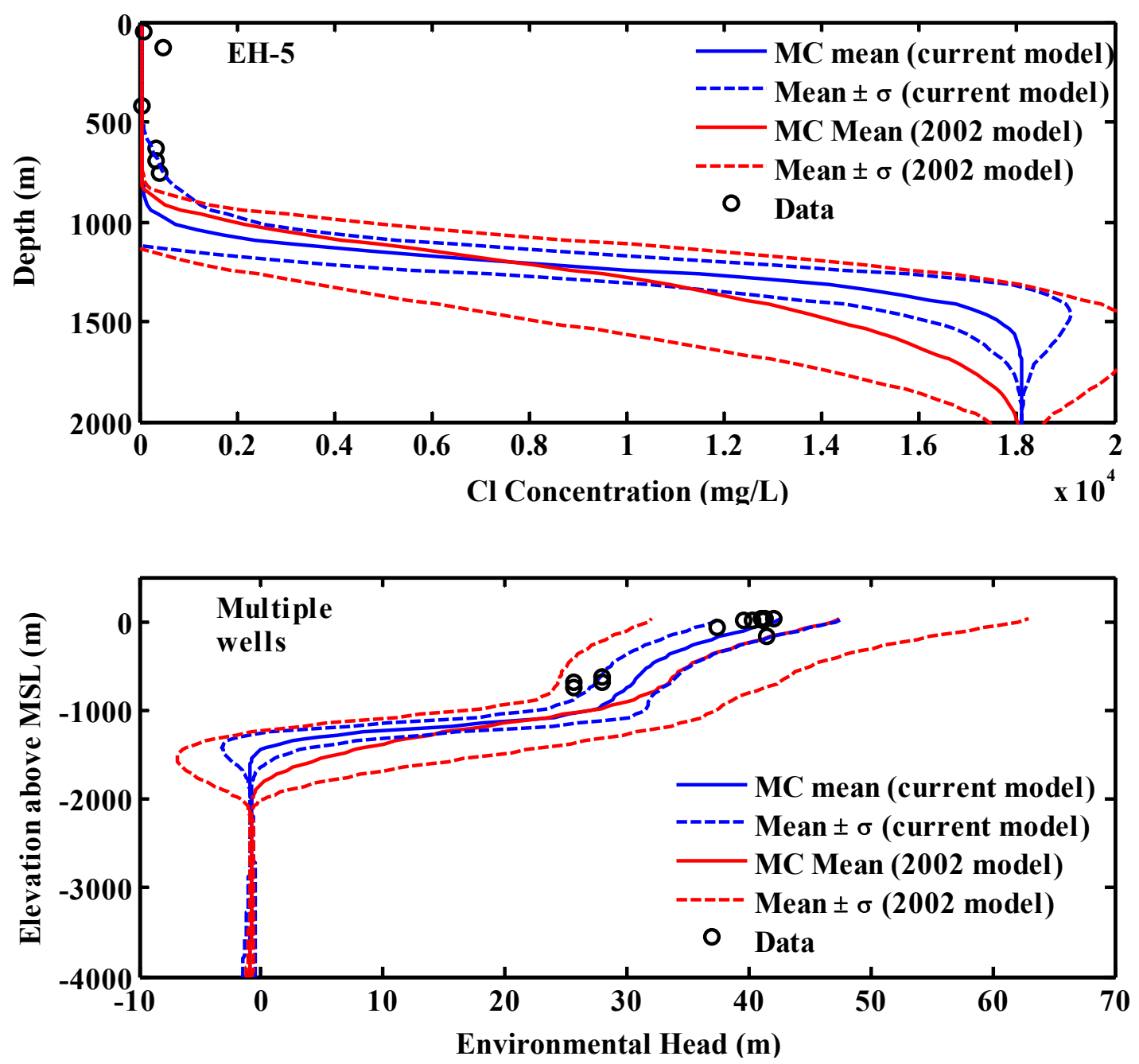

Figure 5.6. Mean \pm one standard deviation for salinity profile at EH-5 and head profile (at a cell where multiple wells are clustered) at Long Shot as obtained from the new model and the 2002 model.

The second important finding is that the new results are encompassed within the original model results. That is, the new MC mean and all of the uncertainty bounds are captured within the original range of possible outputs (i.e., the original MC mean $\pm \sigma$ ). This verifies the original Long Shot model in the sense that the original model did account for much deeper as well as shallower transition zones compared to the MC mean. Also, the match of the MC mean to the head data has improved compared to the 2002 model, and so has the match to the salinity data. Five out of the six salinity points provided from EH-5 are now captured by the MC mean $\pm \sigma$ bounds of the results.

\subsection{Cannikin Flow Results}

Similar to Milrow and Long Shot, the recharge and conductivity values for Cannikin are generated from the MCMC results of 90,000 samples (Figure 4.25b). The 2,000 porosity profiles are generated from the bounds given by Unsworth et al. (2005) and are assigned randomly to the $R-K$ pairs. Figure 5.7 compares the generated distributions of recharge, 
conductivity, and porosity to those used in Hassan et al. (2002). The porosity distribution plotted in the figure is at the cavity horizon. The lowest porosity value in the new distribution (about 0.0096) is about three orders of magnitude higher than the lowest porosity used in the 2002 model which was about $1.0 \times 10^{-5}$. Also, about 88 percent of the realizations ( 230 out of 260) in Hassan et al. (2002) had porosities less than 0.00096 (i.e., more than an order of magnitude lower than the lowest porosity value in the new distribution). About 55 realizations ( $\sim 21$ percent) had porosities less than 0.000096 (i.e., two orders of magnitude lower than the minimum value in the new distribution). The largest porosity value in the distribution used in Hassan et al. (2002), which was about 0.0052, is more than an order of magnitude lower than the largest value in the new distribution, which is about 0.0652 . It should be remembered that for Long Shot and Cannikin, the porosity profiles provided by Unsworth et al. (2005) were scaled down by a factor of about 0.73 for Long Shot and 0.43 for Cannikin to have a porosity value at the top (zero depth) of no more than 40 percent. Thus, if one were to use the original profiles in Unsworth et al. (2005), the generated porosity values would have been higher than the values shown in Figures 5.5 and 5.7. This downward scaling of the porosity is a conservative approach, as it leads to higher velocities and earlier arrival times.
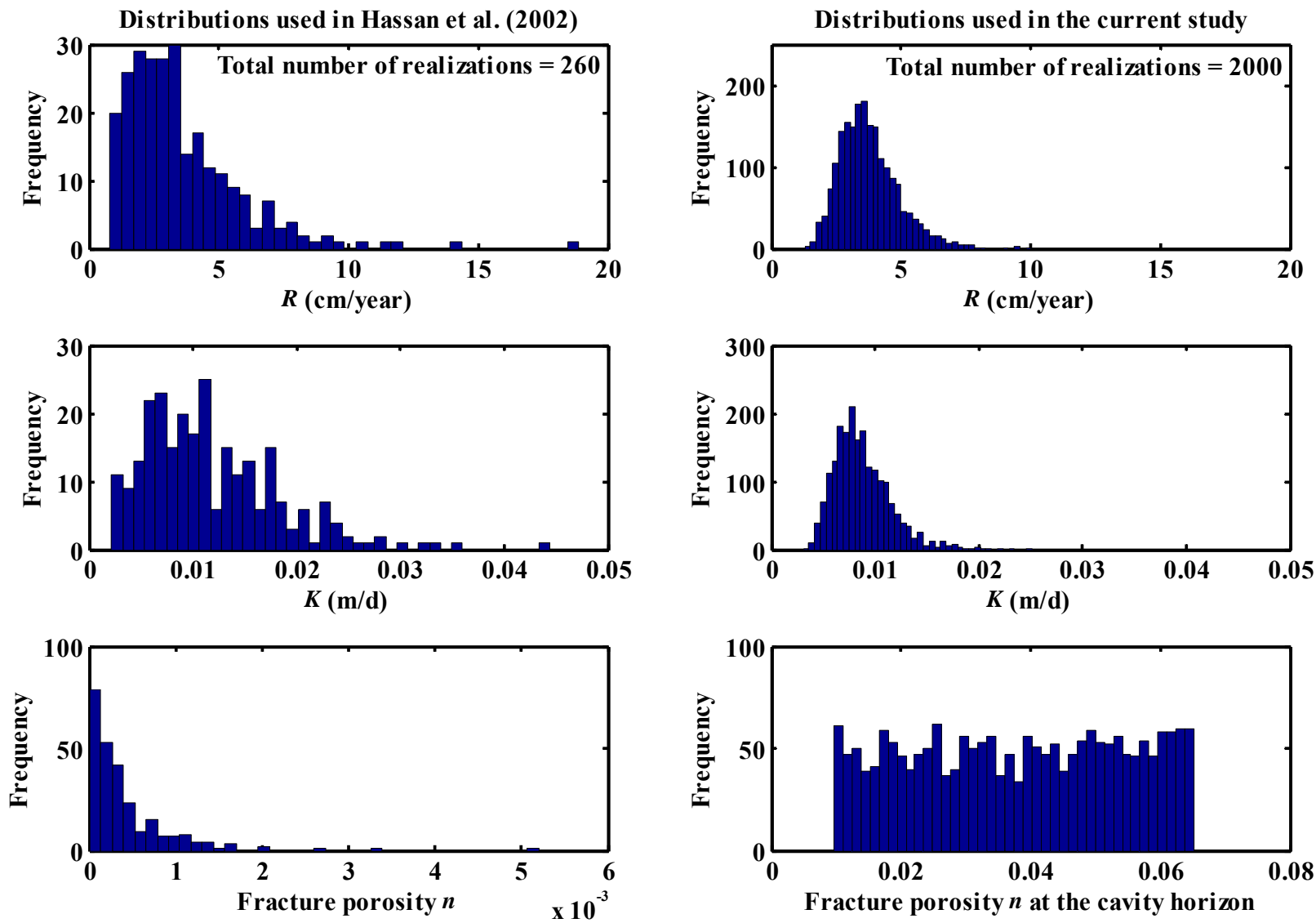

Figure 5.7. Comparison between the distributions of Cannikin parameters used in Hassan et al. (2002) and the distributions used in this study that are based on new data. Fracture porosity distribution in the current model is plotted at the elevation of the test. 
The flow and transport parameters used in Cannikin simulations are summarized in Table 5.4. FEFLOW is used to solve the density driven flow problem for the 2,000 realizations with the generated recharge, conductivity, and porosity (Figure 4.25). Similar to the previous two sites, macrodispersivity values are fixed at $100 \mathrm{~m}$ and $10 \mathrm{~m}$ in the longitudinal and transverse directions, respectively. To assess the uncertainty reduction and verify the original 2002 model, the results of these realizations are analyzed at the UAe-1, UA-1, and HTH-1 locations for salinity and heads (Figures 5.8 and 5.9). A dramatic decrease of uncertainty in the location of the transition zone is achieved by conditioning on the three data sets (Figures 5.8 and 5.9). The uncertainty bounds are very tight in the current model compared to the original range of uncertainty.

Table 5.4. Parameter range for Cannikin flow and transport simulations.

\begin{tabular}{|c|c|c|c|c|c|}
\hline Parameter & Minimum & Mean & Maximum & $\sigma$ & Distribution \\
\hline$R$ & 1.2922 & 3.7655 & 10.0255 & 1.1332 & lognormal \\
\hline$K$ & 0.0031 & 0.0087 & 0.0251 & 0.0027 & lognormal \\
\hline$n$ & $\begin{array}{c}\text { Figure } 4.5 f \text { lower } \\
\text { bound }(m=1)\end{array}$ & $\begin{array}{c}\text { Figure } 4.5 f \text { middle } \\
\text { profile }(m=1.5)\end{array}$ & $\begin{array}{l}\text { Figure } 4.5 f \text { upper } \\
\text { bound }(m=2)\end{array}$ & $\begin{array}{c}\sim 0.0163 \text { at cavity } \\
\text { horizon }\end{array}$ & Uniform \\
\hline$A_{L}$ & - & 100 & - & - & - \\
\hline$A_{T}$ & - & 10 & - & - & - \\
\hline$\alpha_{L}$ & - & 5 & - & - & - \\
\hline$\alpha_{T}$ & - & 0.5 & - & - & - \\
\hline$k$ & - & 0.434 & - & - & - \\
\hline$k_{g}$ & - & $1.26 \times 10^{-7}$ & - & - & - \\
\hline
\end{tabular}

The second observation is that the current results in terms of the mean $\pm \sigma$ are enclosed within the 2002 model results for most of the salinity and head profiles. This verifies the original model and indicates that the original uncertainty built into the model parameters was sufficiently large to cover possibilities of a very shallow as well as a very deep transition zone. The current model yields a much deeper transition zone (mean of the MC realizations) compared to the 2002 model (Figures 5.8 and 5.9). The match in the new results to the salinity and head data is much better, as the salinity data are well captured by the uncertainty bounds and the head data are better matched with the current results. The only exception is the HTH-1 data set, which was better matched in the original model. However, the good match to the data from UAe-1 and UA-1 and the consistency of their data suggest a possibility for data errors at HTH-1.

Figures 5.8 and 5.9 indicate that the new results verify the 2002 model in some aspects and fail to verify the model in others. At UAe-1, the new data and the conditioning on them suggest a lack of verification for portions of the 2002 model salinity distribution. Overall, the new results match the salinity data at UAe-1 better than did the original model, but still failed to enclose all salinity data points within the mean $\pm \sigma$ uncertainty bounds. Although this is a caveat in the new results, compared to the original model, more data points are enclosed by the uncertainty bounds in the new results. In regards to the wells with head data, the new model verifies the original model and does a much better job matching the head data at UA-1. Verification and uncertainty reduction are also achieved at HTH-1, but the new 
model failed to match the head data. Verification and uncertainty reduction are achieved at UAe-1, but both the 2002 model and the current model fail to provide a good match to the head data. This is probably attributed to the fact that the head data at UAe-1 do not provide a clear head profile with depth (like UAe-2 at Milrow or EH-5 at Long Shot), which makes it difficult for the model to match the data.

The Cannikin data sets present a clear example of a case where it is not possible to simultaneously calibrate to or well match the head and chemistry data. This was also a case in the Milrow head and salinity data at UAe-2. Given our inability to calibrate to both head and chemistry simultaneously, the 2002 model favored the heads at Cannikin because some concerns exist regarding the quality of the Cannikin chemistry samples. The new MT data pertaining to the transition zone location were informative at Cannikin where they supported the chemistry data, resulting in new calibrations that favored the chemistry data, but did leave some head values outside the uncertainty bounds of the new model.
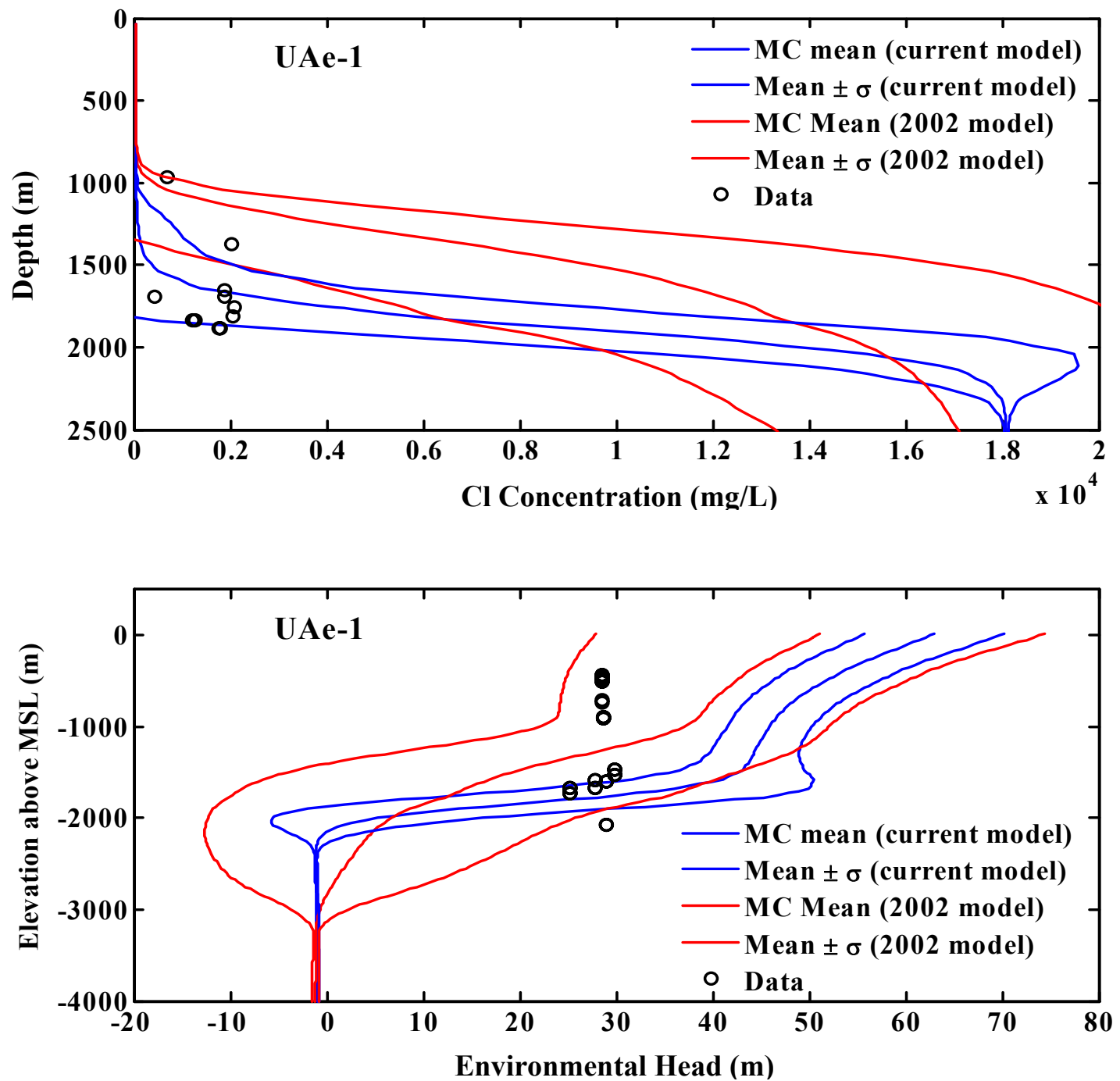

Figure 5.8. Mean \pm one standard deviation for salinity and head profiles at UAe-1 location at Cannikin, as obtained from both the new model and the 2002 model. 

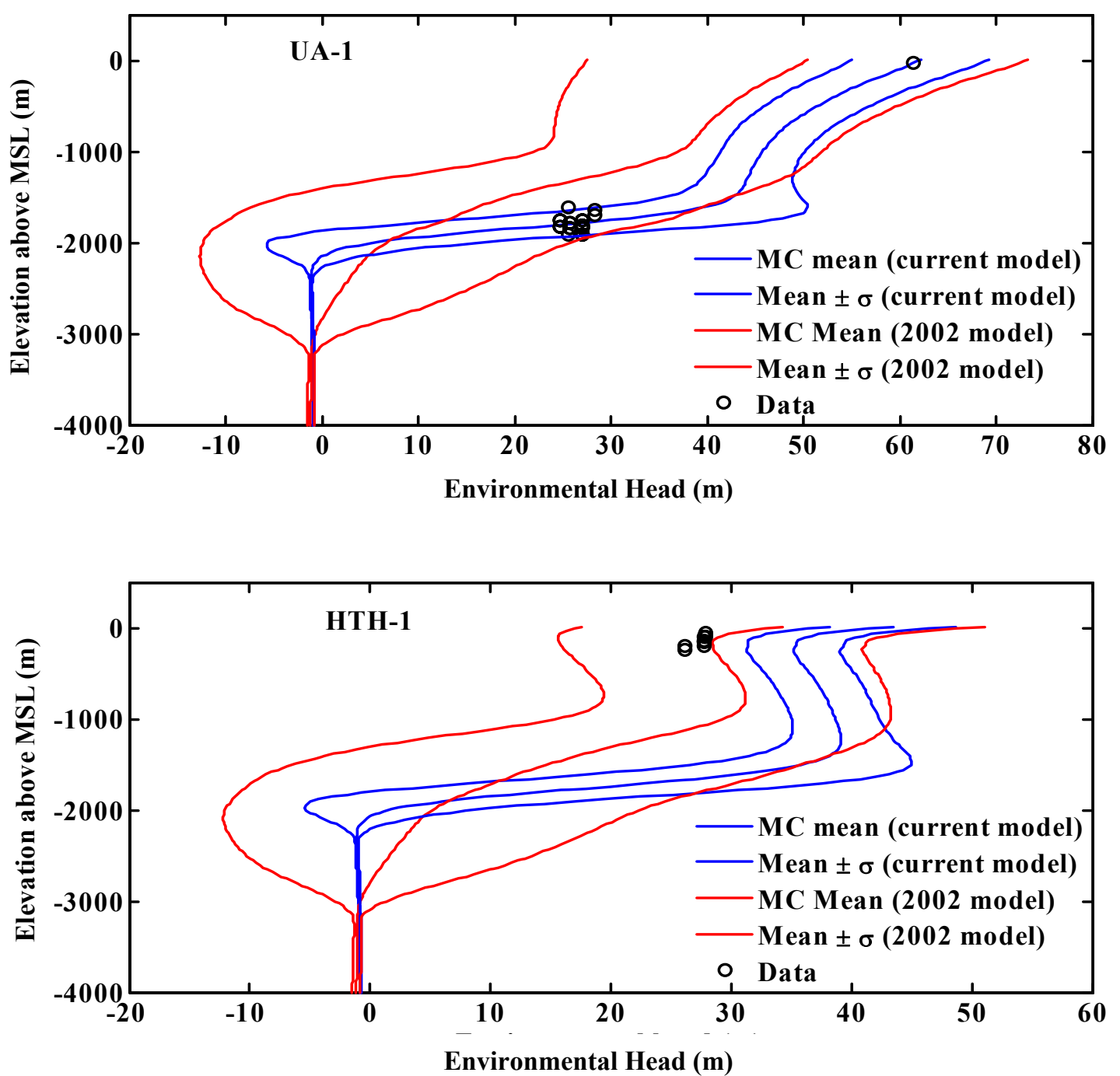

Figure 5.9. Mean \pm one standard deviation for salinity profiles at UA-1 (a) and at HTH-1 (b) at Cannikin, as obtained from both the new model and the 2002 model. 


\section{REVISED TRANSPORT MODELING}

The migration of contaminants from the underground nuclear tests at Amchitka involves a complex system of physical and chemical processes. A diagrammatic representation of the transport model source and processes considered in Hassan et al. (2002) is shown in Figure 6.1. A brief description of the transport processes considered in the original model follows.

The contaminants considered consist of the radionuclides produced by Milrow, Long Shot, and Cannikin and the daughters created by radioactive decay. The nuclides were assumed to initially all be located within the cavity. Distribution upward through the chimney region was neglected. Sampling in the Cannikin post-test hole confirmed that the bulk of radioactivity was contained within the cavity region (Claassen, 1978). In general, the primary effect of spreading the source nuclides through a larger volume would be to disperse and dilute the mass, lowering concentrations.

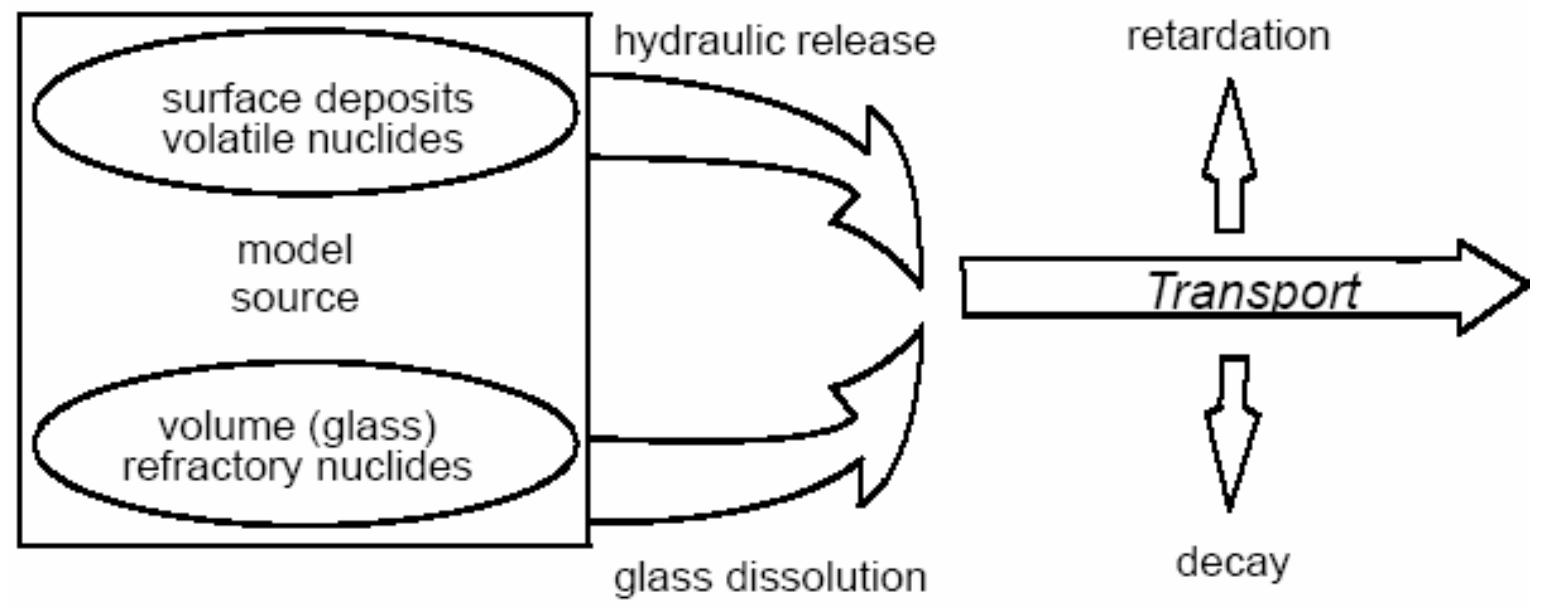

Figure 6.1. Flowchart of the transport model source and processes considered in the 2002 study.

Radionuclides were distributed according to their volatility among surface deposits and volume deposits in nuclear melt glass. Volatile and surface-deposited nuclides were assumed to migrate immediately after the nuclear test. Nuclides within the glass were released according to glass dissolution rates calculated based on volcanic glass dissolution behavior and radionuclide melt glass characteristics.

Once released, the nuclides are subjected to retardation processes. Diffusion of contaminants from fractures into surrounding matrix blocks was also considered. Some radionuclides were retarded by reactions with aquifer materials. Retardation factors were calculated from a surface-based sorption constant for the fractures, based on estimates of fracture aperture and distribution coefficients derived from batch experiments on Amchitka cores. Nuclides were grouped according to general sorptive behavior and assigned the same retardation factor because radionuclide-specific data were not available for many of the contaminants in the Amchitka environment. 
The CRESP efforts did not provide transport-relevant data (except porosity), so the current conceptual transport model remained the same as the 2002 model and all parameters remained unchanged. The parameters related to retarding the radionuclide movement (e.g., mass trapped in nuclear melt glass, matrix diffusion, retardation by sorption and radioactive decay) were not used in this study, as transport velocities were so small that radionuclides did not move far away from the cavity during a simulation time of 2,200 years. This will be discussed in detail in Sections 6.1 through 6.3.

Transport of a nonreactive solute in saturated porous media of constant porosity is described by

$$
\frac{\partial C(\mathbf{x}, t)}{\partial t}+\nabla \cdot[C(\mathbf{x}, t) \mathbf{V}(\mathbf{x})]-\nabla \cdot\left[\mathbf{D}_{h}(\mathbf{x}) \cdot \nabla C(\mathbf{x}, t)\right]=0
$$

where $C(\mathbf{x}, t)$ represents concentration, $\mathbf{V}(\mathbf{x})$ is the velocity vector at location $\mathbf{x}$, and $\mathbf{D}_{\boldsymbol{h}}(\mathbf{x})$ represents the diagonal of the local hydrodynamic dispersion tensor. The components of $\mathbf{D}_{\boldsymbol{h}}(\mathbf{x})$ are given as (Bear, 1972)

$$
\mathbf{D}_{h}(\mathbf{x})=\alpha_{T}|\mathbf{V}(\mathbf{x})| I+\left(\alpha_{L}-\alpha_{T}\right) \frac{\mathbf{V}(\mathbf{x}) \mathbf{V}(\mathbf{x})}{|\mathbf{V}(\mathbf{x})|} I+D^{*} I
$$

where $\alpha_{L}$ and $\alpha_{T}$ are the longitudinal and transverse local dispersivities, $|\mathbf{V}(\mathbf{x})|$ is the magnitude of velocity, $I$ is the three-dimensional identity matrix, and $D^{*}$ is the effective coefficient of molecular diffusion.

Several numerical approaches can be used to solve the transport equation, for example, finite differences, finite elements, method of characteristics, and random walk particle-tracking methods. In the 2002 and also in the current study, the random walk method is used to simulate the transport and evolution of radionuclides in the generated random velocity fields. The radionuclide mass is replaced with a large number of particles $N P$ of equal mass $m$ that are tracked in the space-time domain. We use 100,000 particles in all the transport simulations presented in this study.

The positions of the particles are updated at each time step according to the random walk equation (Kinzelbach, 1988; Tompson and Gelhar, 1990)

$$
\mathbf{x}_{t+\Delta t}=\mathbf{x}_{t}+\left[\mathbf{V}\left(\mathbf{x}_{t}, t\right)+\nabla \cdot \mathbf{D}_{h}\left(\mathbf{V}\left(\mathbf{x}_{t}, t\right)\right)\right]+\left[2 \mathbf{D}_{h}\left(\mathbf{V}\left(\mathbf{x}_{t}, t\right)\right) \Delta t\right]^{1 / 2} \cdot \mathbf{Z}
$$

where $\mathbf{x}_{t+\Delta t}$ is the updated position vector of the particle that was at $\mathbf{x}_{t}$ in the previous time step, $\mathbf{V}\left(\mathbf{x}_{t}, t\right)$ is the velocity vector at the old position at time $t, \mathbf{D}_{\boldsymbol{h}}$ is the local-scale dispersion tensor that is a function of the local velocity, $\Delta t$ is the time step, and $\mathbf{Z}$ is a vector of normally distributed random numbers of zero mean and unit variance. The first term on the right-hand side of Equation (6.3) represents the advective step and the second term adds the effect of the gradients of the dispersion tensor on the particle movement. This latter term is important if sharp fronts exist and whenever the gradient of $\mathbf{D}_{\boldsymbol{h}}$ is significant. The last term represents the contribution of local-scale dispersion and Brownian diffusion to the movement of the particles.

The output velocity fields of the FEFLOW solution are obtained at an irregular finite-element mesh. However, for convenience and simplicity in computations, the velocity values are interpolated on a uniform grid. The particle velocities needed in Equation (6.3) are 
then obtained by using a bilinear interpolation scheme using the four flux values surrounding the particle and then dividing by the porosity of the cell where the particle resides.

\subsection{Milrow Transport Results}

The 24 radionuclides (parents and daughters) chosen for investigation were grouped into six solute classes based on their ratio of hydraulic release to geochemical release and retardation factor (Hassan et al., 2002). The particle-tracking transport simulations are performed here for one of these six classes. The first class (consisting of ${ }^{3} \mathrm{H},{ }^{14} \mathrm{C},{ }^{36} \mathrm{Cl},{ }^{85} \mathrm{Kr}$ ) has no mass trapped in the nuclear glass and as such, 100 percent of the mass is made available to move with the groundwater from time zero. This class also had no retardation. Transport simulations are conducted for a total simulation time of 2,200 years using a time step of about 1,000 days. This time step is 10 times larger than that used for Milrow in Hassan et al. (2002) since the velocities here are much smaller than in the 2002 study. Longitudinal local dispersivity is taken as $5.0 \mathrm{~m}$ and transverse dispersivity is taken as $0.5 \mathrm{~m}$.

Running the transport simulations for the 2,000 flow realizations of Milrow and considering only advection and dispersion processes resulted in no breakthrough in 2,200 years. None of the 100,000 particles reached the seafloor during this simulation time. Again, the particles only experienced advection and dispersion. Matrix diffusion and radioactive decay have not been incorporated into the transport simulation, despite their known occurrence. As alluded to in Section 5, the porosity profiles provided by Unsworth et al. yield porosity values orders of magnitude higher than the range implemented in Hassan et al. (2002). This high fracture porosity yields very slow flow velocities that only move the particles few hundred meters away from the cavity in 2,200 years.

To illustrate this, the realization with the highest fracture porosity from the 2002 model is rerun (Figure 6.2a). The recharge-conductivity ratio for this realization is then used in a realization of the new model (Figure $6.2 \mathrm{~b}$ ) with a porosity profile matching the lower bound provided by Unsworth et al. (2002). This would thus produce one of the fastest realizations in the current model. The maximum distance traveled by particles in the new model realization is less than $400 \mathrm{~m}$, whereas the slowest realization in the original model had a maximum travel distance of about $3,000 \mathrm{~m}$. Since all remaining realizations of the new model have higher porosity than the test case (Figure 6.2b), it is expected that no breakthrough would occur in any individual realization during the simulation time frame.

As a check on the computational algorithm, the groundwater flux values from the current flow realizations are used but are combined with the 2002 porosity distribution. Three hundred realizations are chosen at random from the 2,000 realizations and are also randomly assigned the porosity values used in the 300 realizations of the 2002 model. Transport simulations are then conducted using these flow fields and porosity values, and the resulting breakthrough curves (MC mean, $<Q(t)>$, standard deviation, $\sigma_{Q}$, and mean plus two standard deviations, $<Q(t)>+2 \sigma_{Q}$ ) are obtained (Figure 6.3), accounting only for advection and dispersion. For comparison purposes, the breakthrough curves of the 2002 model with only advection and dispersion are shown.

The current groundwater flux realizations with the new porosity data provided by Unsworth et al. (2005) yield essentially no breakthrough in 2,200 years. When combined with the 2002 model porosities, the current flow realizations yield mean mass flux exceeding that of the original 2002 model. This is probably due to the deeper transition zone simulated 
in the new model. This result shows that the numerical models are performing well and that the no-breakthrough result is indeed attributed to the higher porosities developed through the analysis of data collected by CRESP.

a)

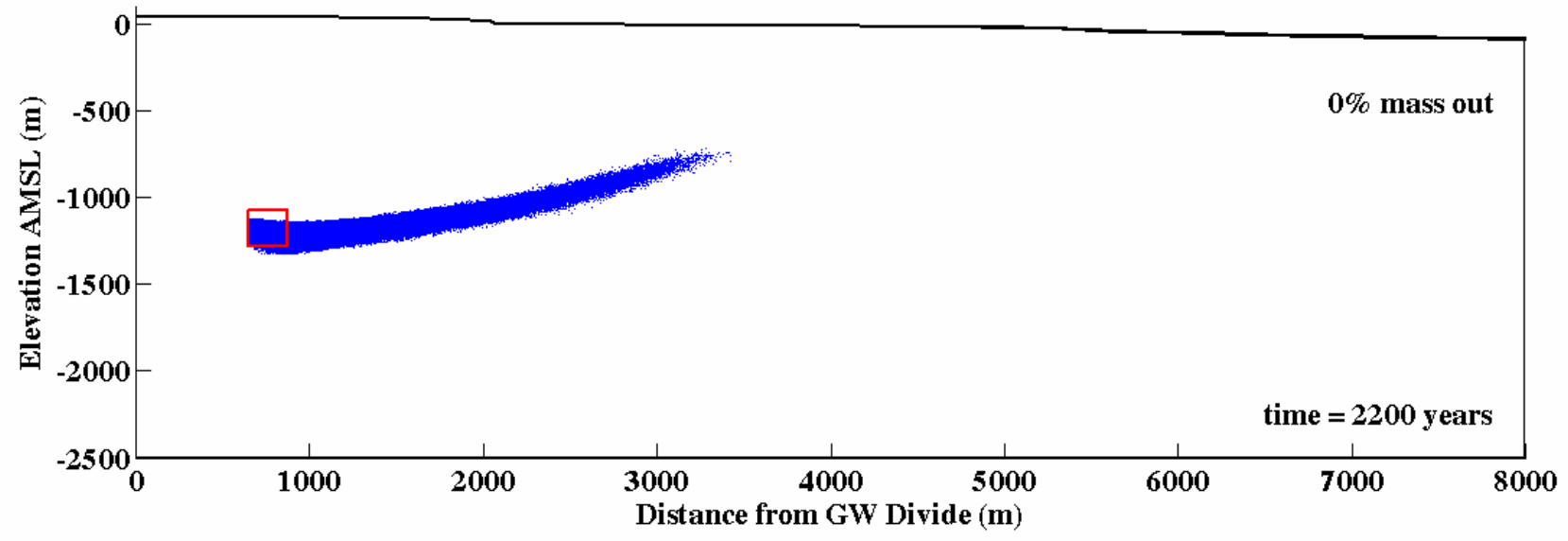

b)

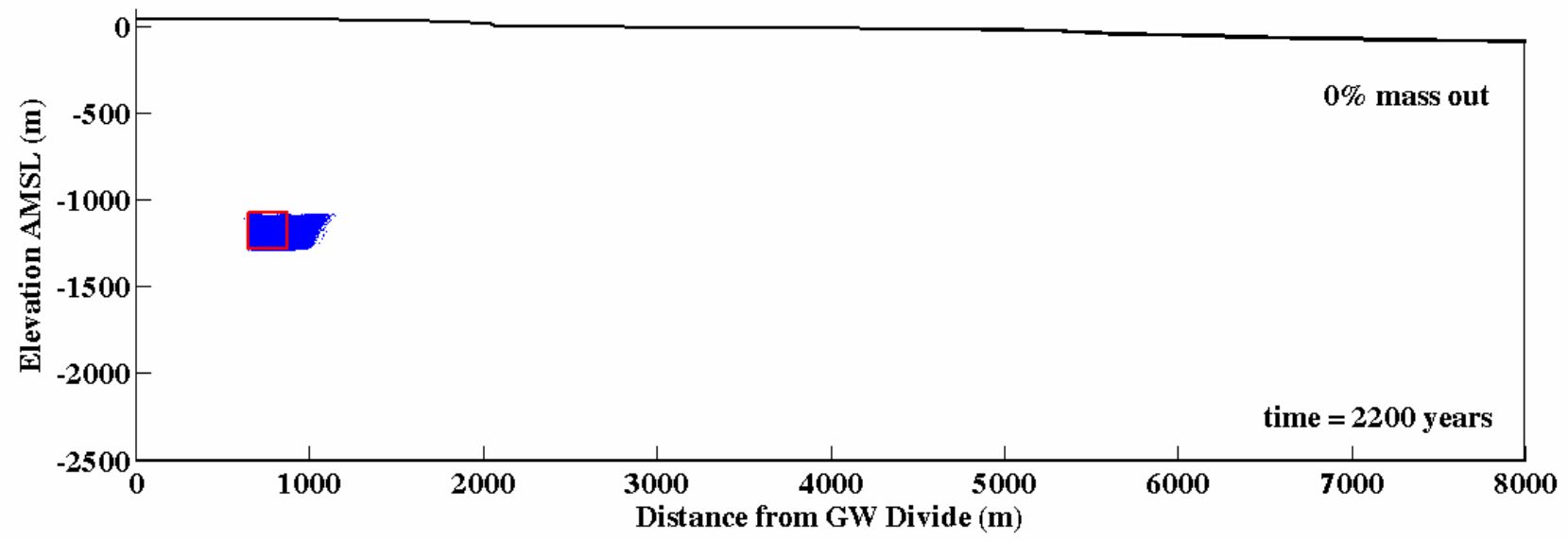

Figure 6.2. Particle positions at 2,200 years from two realizations: a) a 2002 model realization with the highest porosity (i.e., slowest realization), and b) a current model realization with the same $R / K$ and with the lower porosity bound shown in Figure 4.5. 

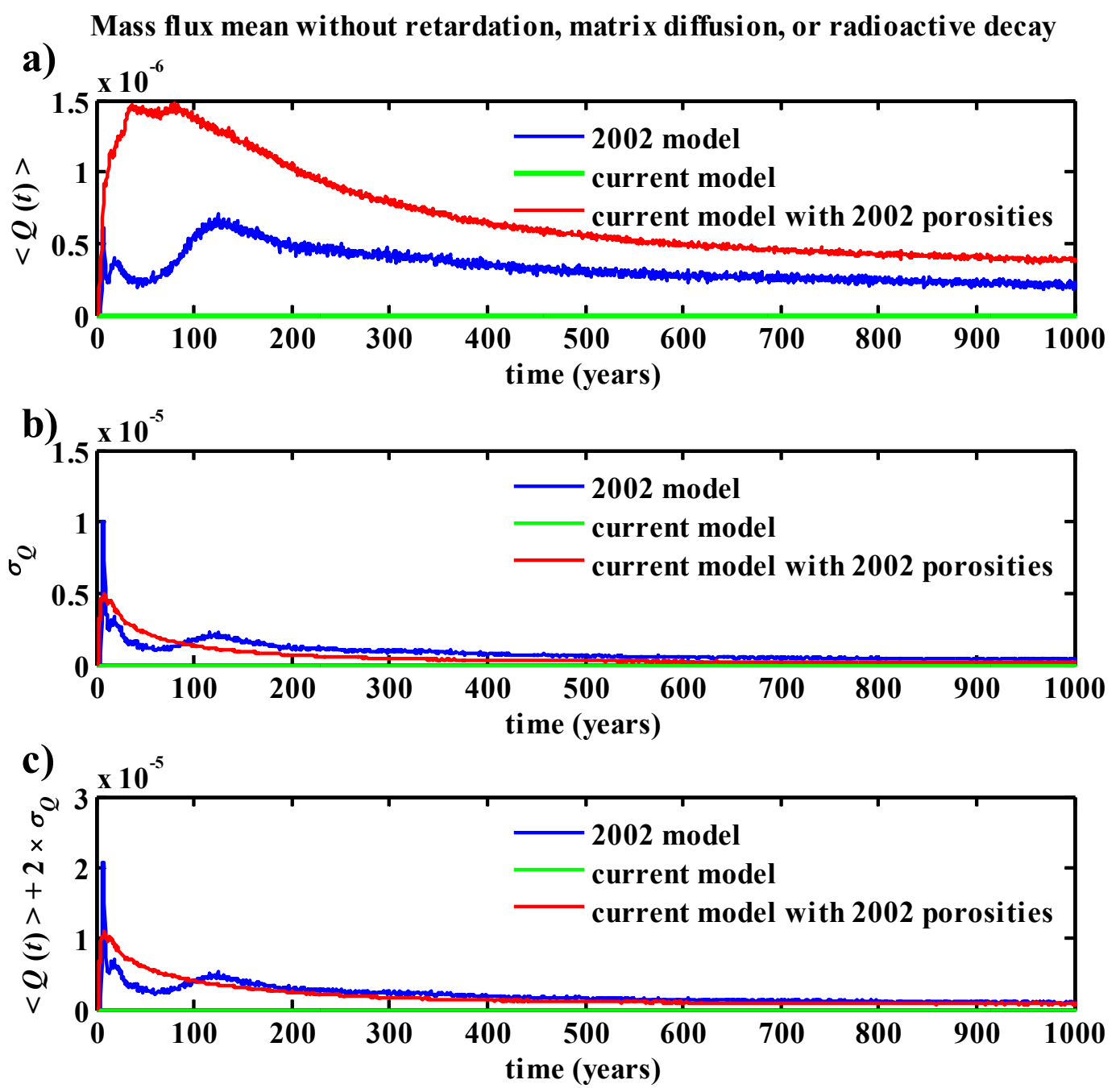

Figure 6.3. Mass flux moments as computed with only advection and dispersion. Comparisons are made between the 2002 model (blue), the current model (green), and the current model with 2002 porosities (red).

The mean radionuclide mass flux plus two standard deviations, $<Q>+2 \sigma_{Q}$, obtained with the new model combined with the 2002 model porosities, is still lower than the same value obtained from the 2002 model (Figure 6.3c). The peak of the plotted quantity in the current model using 2002 model porosities is about one half the peak of the same quantity in the 2002 model. This serves as another demonstration of model verification as the 2002 model output for mass flux encountered a wide range of uncertainty and the new outcome (assuming we take original porosities) fits within the bounds of the 2002 model output. The uncertainty shown for the current model (Figure 6.3b) stems primarily from the fracture porosity uncertainty adopted from the 2002 model for the sake of this figure. This masks the reduction in $R / K$ uncertainty realized in the new model, as well as ignores the uncertainty reduction gained by the new CRESP-derived porosity distribution.

A final check on the Milrow transport model is to compare the velocities obtained from the current model to the velocities encountered in the 2002 model. A cross section at about $200 \mathrm{~m}$ downgradient from the cavity at Milrow is selected (Figure 6.4). The 
groundwater fluxes and velocities at this section are obtained for all realizations and analyzed (Figure 6.5 and 6.6).
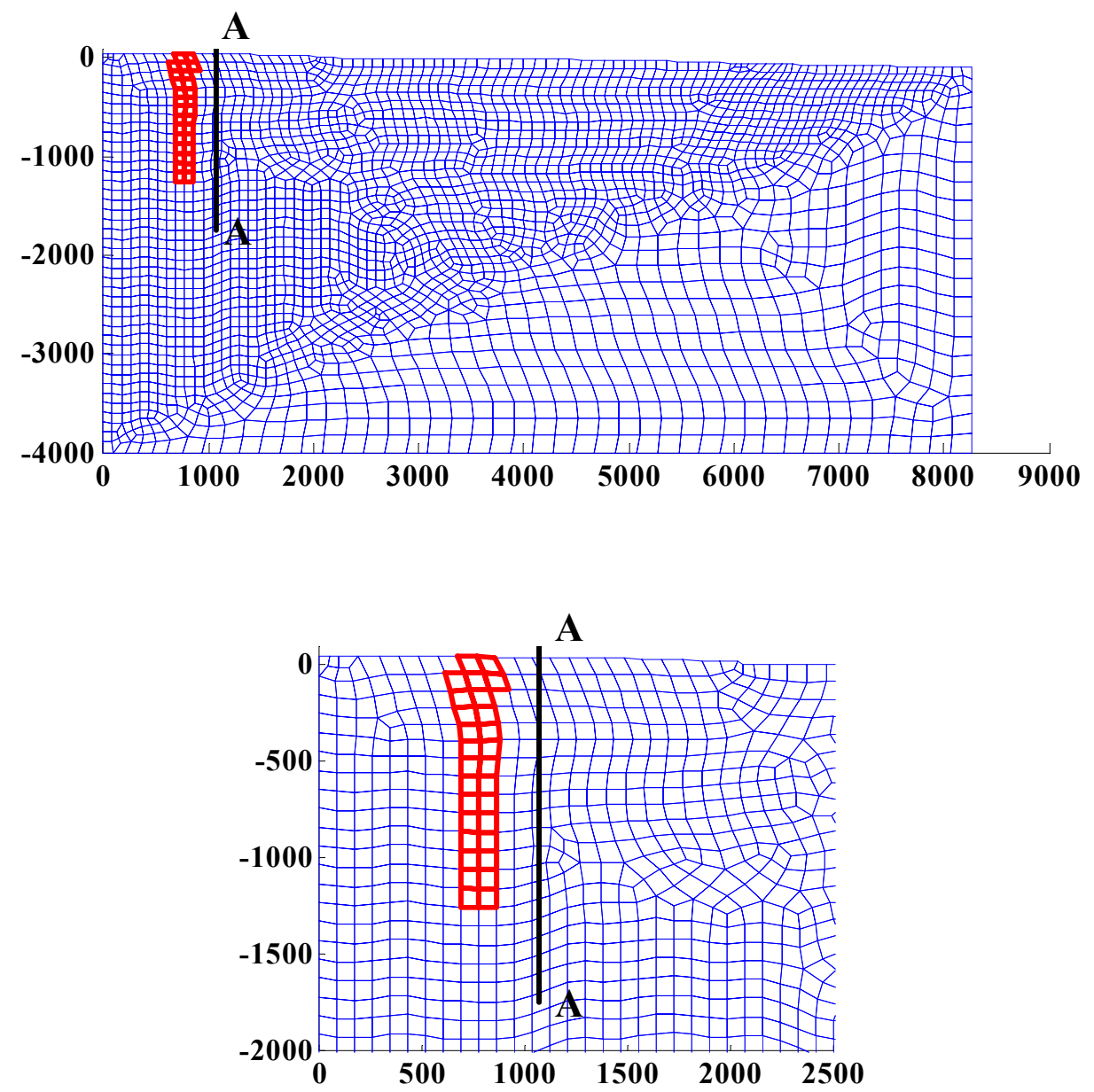

Figure 6.4 Milrow domain and mesh configuration showing the cavity and chimney location and the cross section A-A at which fluxes and velocities are computed for next two figures. The lower plot is a zoom-in close to the A-A cross section.

The groundwater fluxes from the two models are very similar (Figure 6.5). The only difference is the slightly tighter range of fluxes obtained in the current model, which is expected because the conductivity and recharge ranges have been reduced through conditioning on new data. It is interesting though to note that the range reduction occurred more from the lower end of the flux distribution than from the upper end. This is more pronounced for the magnitude of the flux vector, $q=|\mathbf{q}|=\sqrt{q_{x}^{2}+q_{z}^{2}}$, where the lower end of the distribution was trimmed by an order of magnitude (from $1.68 \times 10^{-7}$ to $1.16 \times 10^{-6}$ $\mathrm{m} / \mathrm{d}$ ), whereas the upper end was only reduced by a factor of 0.8 (from $3.51 \times 10^{-4}$ to $2.85 \times$ $10^{-4} \mathrm{~m} / \mathrm{d}$ ). This result provides another verification for the original model and indicates that the range of groundwater fluxes considered in Hassan et al. (2002) did cover the physically 
plausible range that may be encountered beneath the island. The range of groundwater fluxes obtained after conditioning on the CRESP data fits within the original 2002 model range, thereby indicating that the 2002 model captured the range of fluxes.
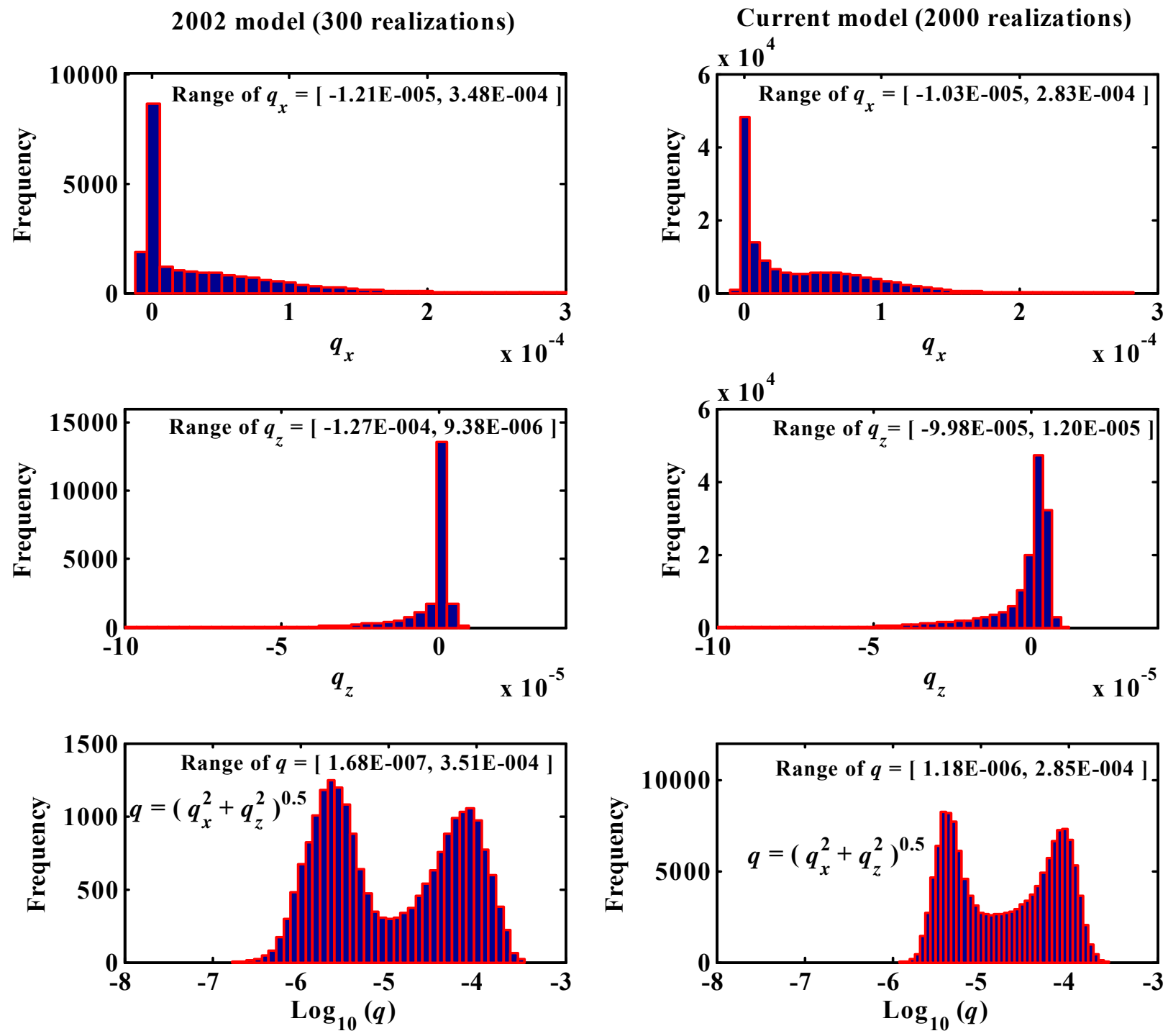

Figure 6.5. Distribution of Milrow groundwater fluxes at section A-A shown in Figure 6.4 and for all realizations. Horizontal flux (a), vertical flux (b), and magnitude of the flux vector (c) are shown.

Flow velocities are obtained by dividing the flux values at each model element by the corresponding porosity (Figure 6.6). On average, the velocities in the current model are at least three orders of magnitude lower than the 2002 model velocities. This implies that if a 2002 model particle were to hypothetically arrive after five years, the same particle would need about 5,000 years to arrive at the seafloor using the current model based on the new CRESP data. 

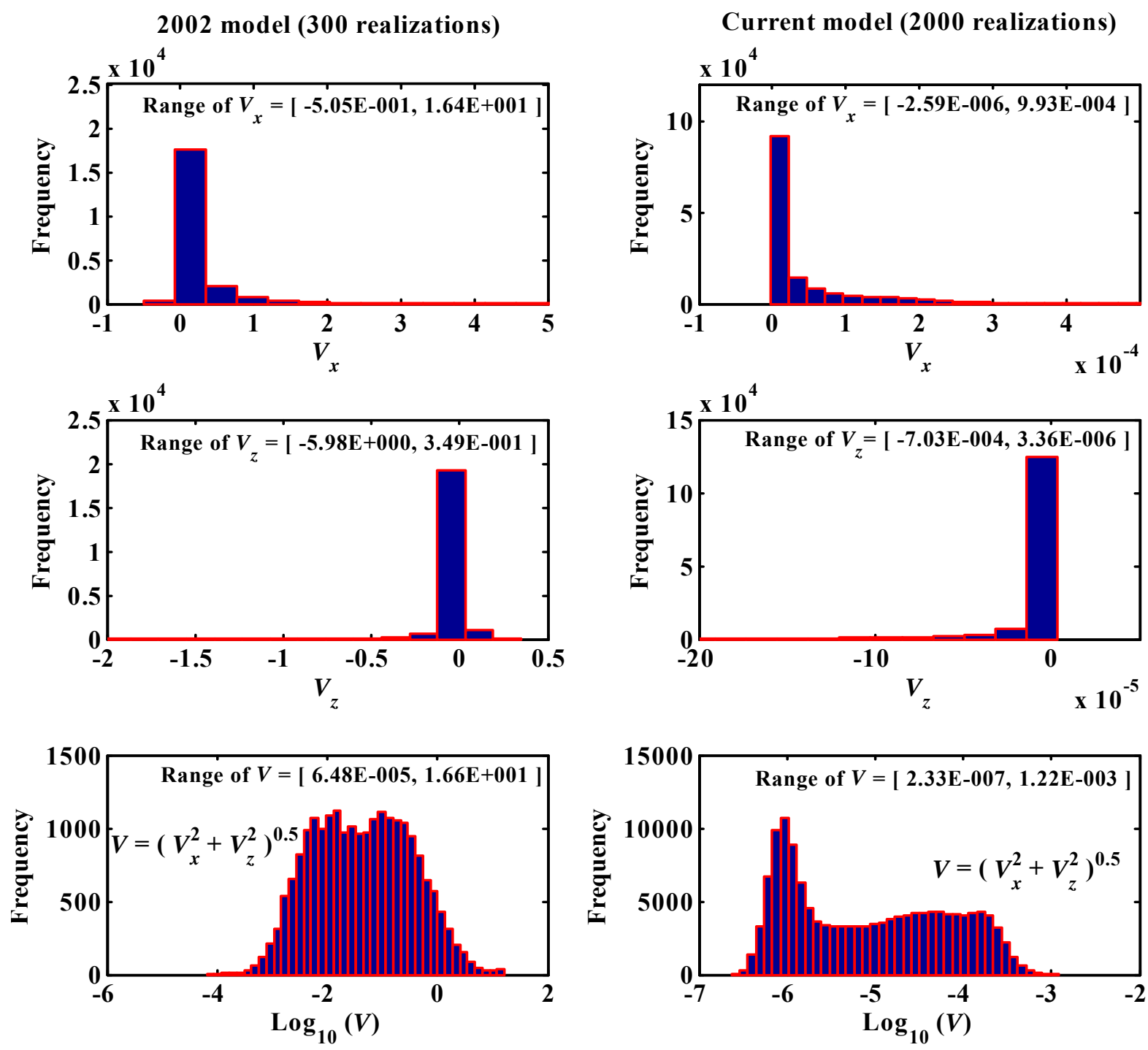

Figure 6.6. Distribution of Milrow groundwater velocities at section A-A shown in Figure 6.4 and for all realizations. Horizontal velocity (a), vertical velocity (b), and magnitude of the velocity vector (c) are shown.

\subsection{Long Shot Transport Results}

Similar to the Milrow site, the particle-tracking transport simulations are performed here for one of the six radionuclide classes considered in Hassan et al. (2002). The first class (consisting of ${ }^{3} \mathrm{H},{ }^{14} \mathrm{C},{ }^{36} \mathrm{Cl},{ }^{85} \mathrm{Kr}$ ) has no mass trapped in the nuclear melt glass, and as such, 100 percent of the mass is made available to move with the groundwater from time zero. This class also had no retardation. Transport simulations are conducted with two transport mechanisms: advection and dispersion. Matrix diffusion and radioactive decay are not included in the simulations. The total simulation time is 2,200 years and the time step is about 250 days. This time step is about four times smaller than at Milrow site. This is due to 
the fact that the Long Shot cavity is very shallow as compared to Milrow, and as such, particles released in the cavity experience the high-velocity region in the freshwater lens above the transition zone for many of the realizations considered. The selection of the time step size for the three sites is determined by analyzing the velocity magnitude at the seepage face for all individual realizations. The maximum value of the velocity at the seepage face is determined for each realization and then the absolute maximum of all these maxima is used to compute the time step such that when multiplied together, they result in a step size smaller than the grid size $(100 \mathrm{~m})$. Similar to Milrow, the longitudinal local dispersivity is taken as $5.0 \mathrm{~m}$ and transverse dispersivity is taken as $0.5 \mathrm{~m}$.

Running the transport simulations for the 2,000 flow realizations of Long Shot and considering only advection and dispersion processes resulted in no breakthrough in 2,200 years. None of the 100,000 particles reached the seafloor during this simulation time. Again the particles only experienced advection and dispersion. Matrix diffusion and radioactive decay have not been incorporated into the transport simulation. As alluded to in Section 5, the porosity profiles provided by Unsworth et al. yield porosity values orders of magnitude higher than the range implemented in Hassan et al. (2002). This high fracture porosity yields very slow flow velocities that only move the particles a few hundred meters away from the cavity in 2,200 years.

A cross section is selected at the Long Shot domain at about $200 \mathrm{~m}$ downgradient from the edge of the cavity and chimney (line marked B-B in Figure 6.7). Groundwater discharges at the different model elements crossing this line are analyzed for all of the 2,000 realizations and also for all the 240 realizations used in Hassan et al. (2002). Groundwater velocities are then obtained by dividing the fluxes by the corresponding element porosity.

Similar to Milrow, the groundwater fluxes are comparable between the 2002 model and the current model (Figure 6.8). The current model, though, has a tighter range of values compared to the 2002 model. For example, the horizontal flux, $q_{x}$, varies between $-1.57 \times 10^{-5}$ and $4.47 \times 10^{-4} \mathrm{~m} / \mathrm{d}$ in the 2002 model, whereas the range in the current model is from $-7.7 \times 10^{-6}$ to $2.27 \times 10^{-4} \mathrm{~m} / \mathrm{d}$. The ranges for the vertical flux component, $q_{z}$, and for the magnitude of the flux vector, $q$, are very similar in the two models (Figure 6.8).

Unlike the Milrow case, the distribution of the flux values $(q)$ is equally and slightly trimmed from both ends of the distribution through conditioning on the new data. The lower end of the distribution increased by a factor of about 1.8 and the upper end decreased by a factor of about 0.5. At Milrow, the lower end is trimmed (increased) by an order of magnitude, whereas the upper end decreased by a factor of about 0.8 . 

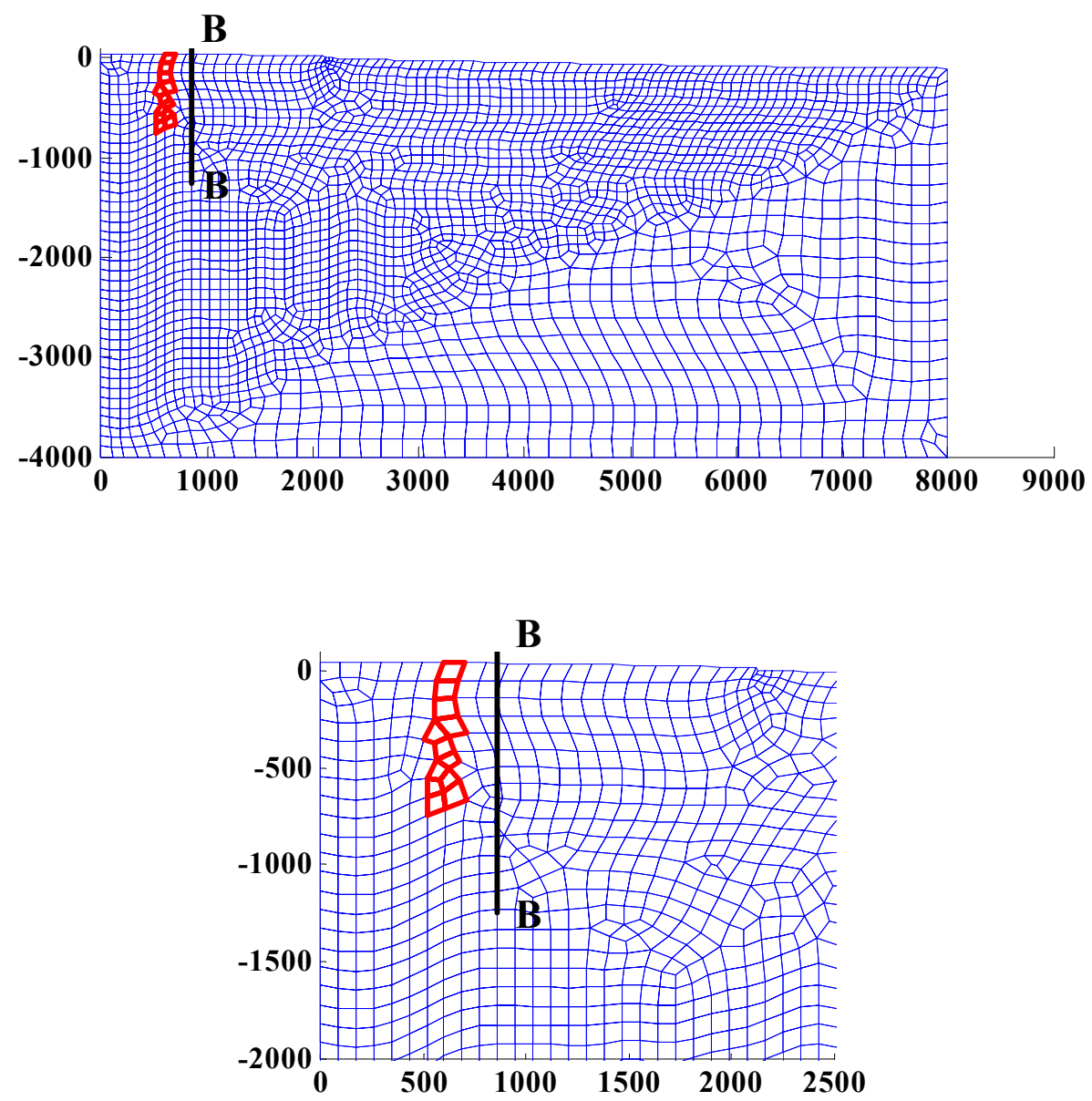

Figure 6.7 Long Shot domain and mesh configuration showing the cavity and chimney location and the cross section B-B at which fluxes and velocities are computed for next two figures. The lower plot is a zoom-in close to the B-B cross section. 

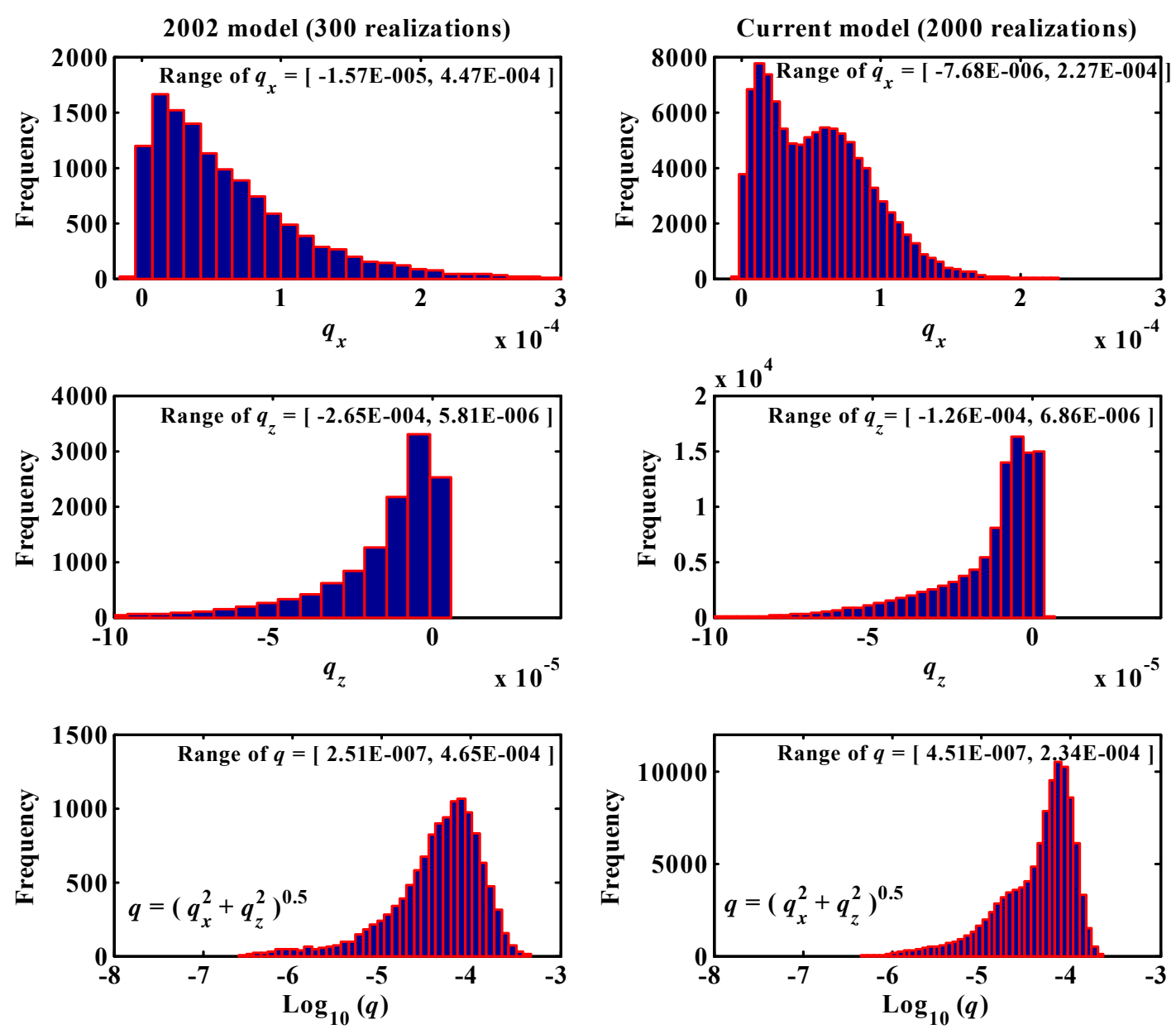

Figure 6.8. Distribution of Long Shot groundwater fluxes at section B-B shown in Figure 6.7 and for all realizations. Horizontal flux (a), vertical flux (b), and magnitude of the flux vector (c) are shown.

The horizontal velocity component, $V_{x}$, is about four orders of magnitude lower in the current model than the 2002 model (Figure 6.9). Similarly, the vertical velocity component, $V_{z}$, is more than four orders of magnitude lower than in the 2002 model. The resulting velocity vector has a magnitude ranging from $2.96 \times 10^{-4}$ to $11.8 \mathrm{~m} / \mathrm{d}$ in the 2002 model and from $7.7 \times 10^{-8}$ to $4.3 \times 10^{-4} \mathrm{~m} / \mathrm{d}$ in the current model. So, the highest velocity in the current model is very close to the lowest velocity of the 2002 model. The entire velocity distribution has been shifted about four orders of magnitude toward the lower side (Figure 6.9) using the new CRESP data. This explains the absence of breakthrough as particles move very slowly away from the cavity and need thousands of years to reach the seafloor. 
2002 model (300 realizations)
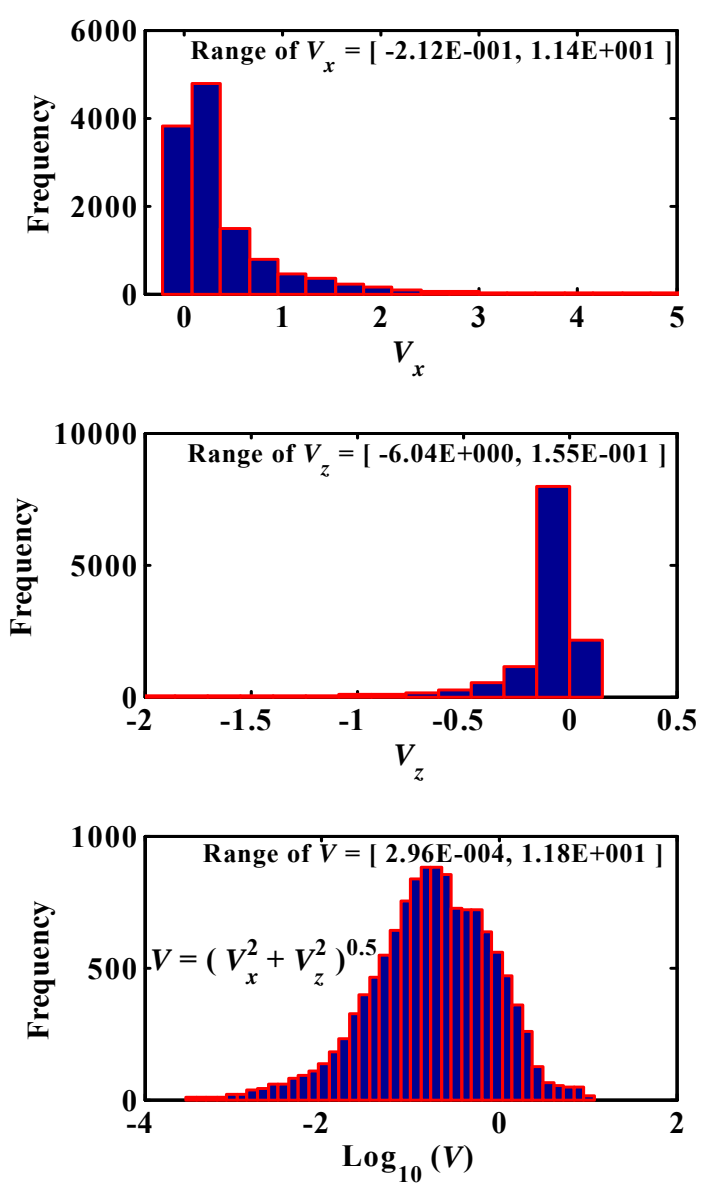
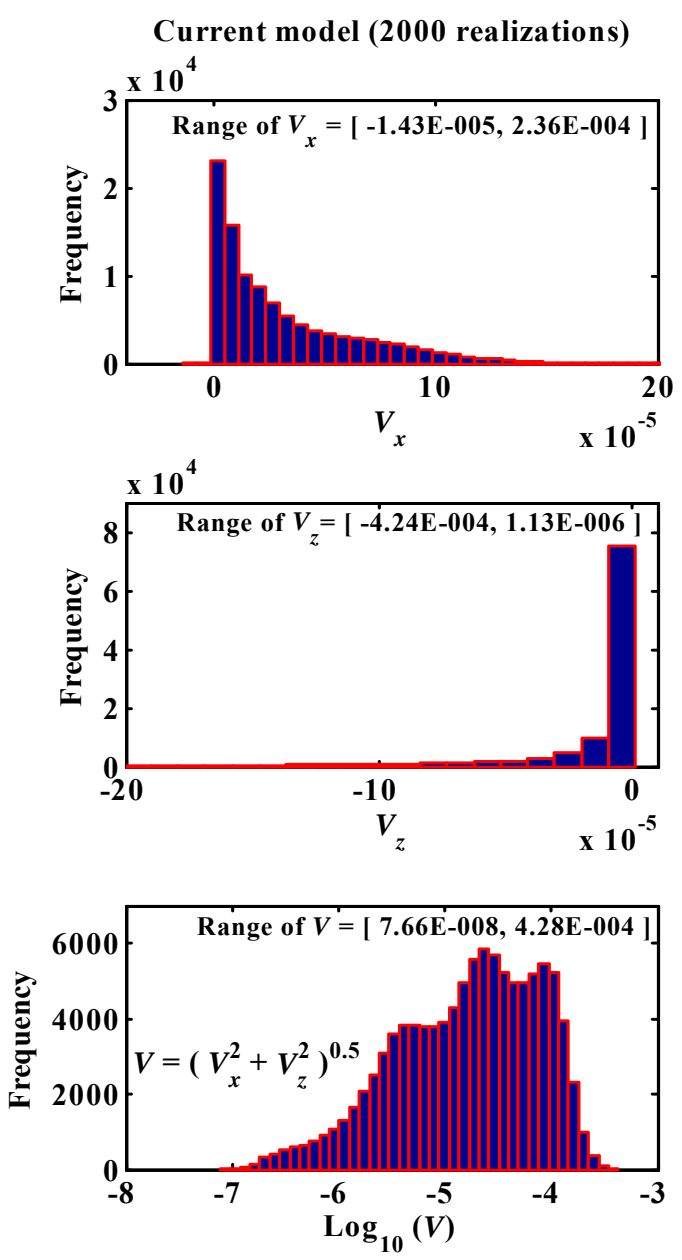

Figure 6.9. Distribution of Long Shot groundwater velocities at section B-B shown in Figure 6.7 and for all realizations. Horizontal velocity (a), vertical velocity (b), and magnitude of the velocity vector (c) are shown.

\subsection{Cannikin Transport Results}

The particle-tracking transport simulations are performed here for one of the six radionuclide classes considered in Hassan et al. (2002). The first class (consisting of ${ }^{3} \mathrm{H},{ }^{14} \mathrm{C}$, ${ }^{36} \mathrm{Cl},{ }^{85} \mathrm{Kr}$ ) has no mass trapped in the nuclear melt glass, and as such, 100 percent of the mass is made available to move with the groundwater from time zero. This class also had no retardation. Transport simulations are conducted with two transport mechanisms: advection and dispersion. Matrix diffusion and radioactive decay are not included in the simulations. The total simulation time is 2,200 years and the time step is about 1,000 days. This time step is similar to the one used for Milrow. The longitudinal local dispersivity is taken as $5.0 \mathrm{~m}$ and transverse dispersivity is taken as $0.5 \mathrm{~m}$.

Running the transport simulations for the 2,000 flow realizations of Cannikin and considering only advection and dispersion processes resulted in no breakthrough in 2,200 years. None of the 100,000 particles reached the seafloor during this simulation time. 
Cannikin has the deepest cavity and, as such, the travel distance from the cavity to the seafloor is longest for Cannikin. If particles did not reach the seafloor at Milrow and Long Shot, then this is expected to be the case at Cannikin.

A cross section is selected for groundwater flux and velocity comparisons at about $200 \mathrm{~m}$ downgradient from the edge of the Cannikin cavity (Figure 6.10, line C-C). Groundwater discharges at the different model elements crossing this line are analyzed for all of the 2,000 realizations and the 260 Cannikin realizations used in Hassan et al. (2002). Groundwater velocities are then obtained by dividing the fluxes by the corresponding element porosity.

The groundwater fluxes are comparable between the 2002 model and the current model (Figure 6.11). The current model, though, has a tighter range of values compared to the 2002 model. For example, the horizontal flux, $q_{x}$, varies between $-5.52 \times 10^{-5}$ and $6.01 \times 10^{-4} \mathrm{~m} / \mathrm{d}$ in the 2002 model, whereas the range in the current model is from $-4.38 \times 10^{-6}$ to $3.34 \times 10^{-4} \mathrm{~m} / \mathrm{d}$. The ranges for the vertical flux component, $q_{z}$, and for the magnitude of the flux vector, $q$, are very close between the two models (Figure 6.11).

Unlike the Milrow case, the distribution of the flux values $(q)$ has been equally and slightly trimmed from both ends of the distribution after conditioning on the new data. The lower end of the distribution has increased by a factor of about 3.3 and the upper end has decreased by a factor of about 0.55 . The distribution of $q$ in Cannikin and Milrow is bimodal in both the current model and the 2002 model. This is attributed to the length of the cross section used for flux comparisons. The line is selected to start at the top of the model domain and to extend to about $500 \mathrm{~m}$ below the cavity. Since at Long Shot, the cavity is shallower than at Milrow and Cannikin, line B-B encountered mostly high fluxes in the freshwater lens in most realizations, thereby yielding a single-mode distribution of flux. For Milrow and Cannikin, the line extends deeply in the domain such that the lower part of the cross section encounters mostly low fluxes below the transition zone, while the upper part of the section encounters higher fluxes above the transition zone. This results in the values obtained over all realizations clustered around high freshwater zone fluxes and low saltwater zone fluxes leading to the bimodal distributions (Figures 6.5 and 6.11). 

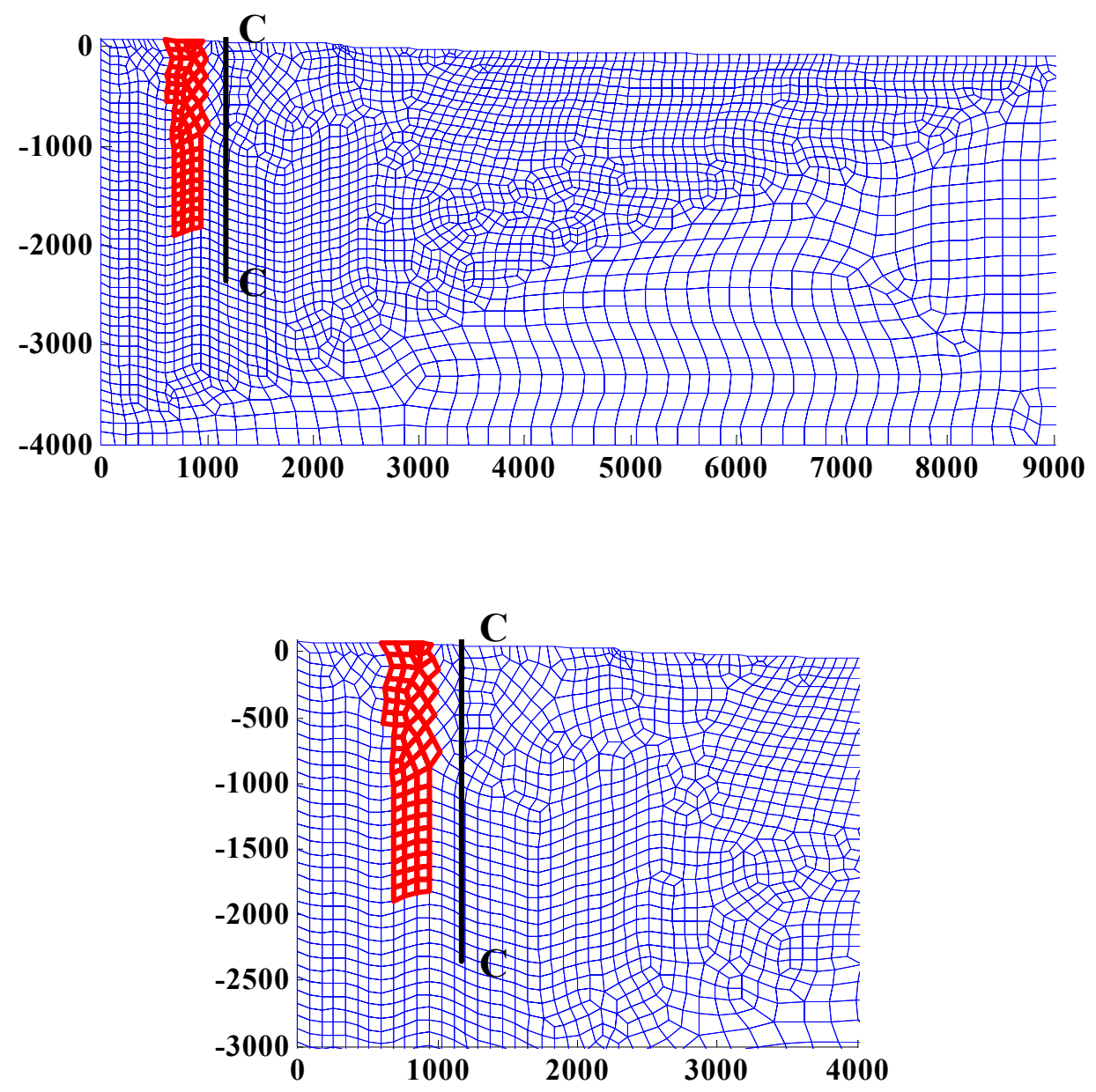

Figure 6.10. Cannikin domain and mesh configuration showing the cavity and chimney location and the cross section $\mathrm{C}-\mathrm{C}$ at which fluxes and velocities are computed for the next two figures. The lower plot is a zoom-in close to the $\mathrm{C}-\mathrm{C}$ cross section. 

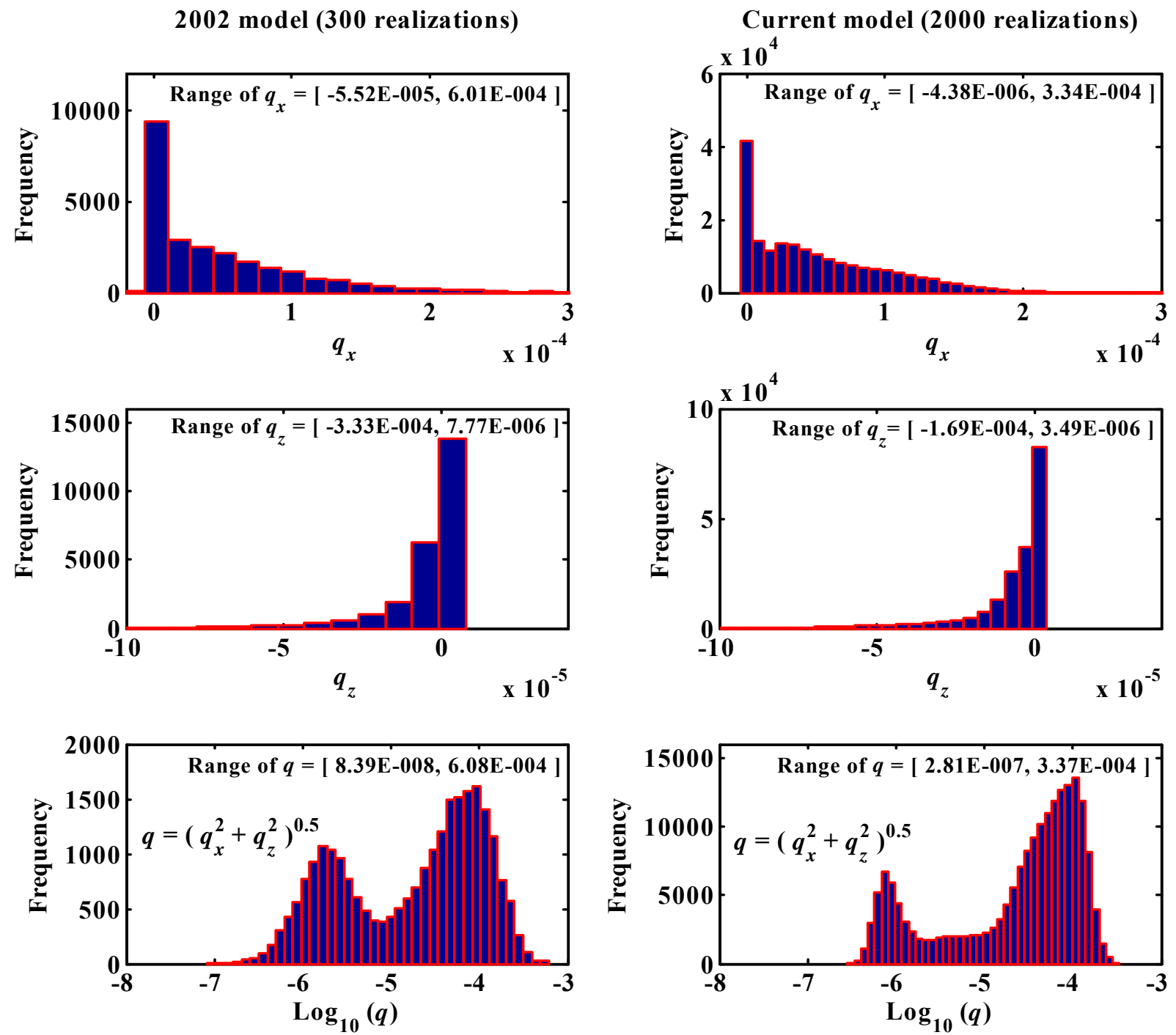

Figure 6.11. Distribution of Cannikin groundwater fluxes at section C-C shown in Figure 6.10 and for all realizations. Horizontal flux (a), vertical flux (b), and magnitude of the flux vector (c) are shown.

The groundwater velocity distributions for Cannikin (Figure 6.12) indicate the current model has velocities many orders of magnitude lower than Hassan et al.'s (2002) model. The $x$-component of the velocity, $V_{x}$, ranges from -0.365 to $13.8 \mathrm{~m} / \mathrm{d}$ in the 2002 model and from $-4.42 \times 10^{-6}$ to $1.69 \times 10^{-4} \mathrm{~m} / \mathrm{d}$ in the current model. The range of the vertical $z$-component of the velocity was $[-5.65,0.165 \mathrm{~m} / \mathrm{d}]$ in 2002 and in the current model it became $\left[-2.56 \times 10^{-4}\right.$, $\left.5.36 \times 10^{-7} \mathrm{~m} / \mathrm{d}\right]$. As a result, the distribution of the magnitude of the velocity vector shifted about four orders of magnitude to lower velocities. 

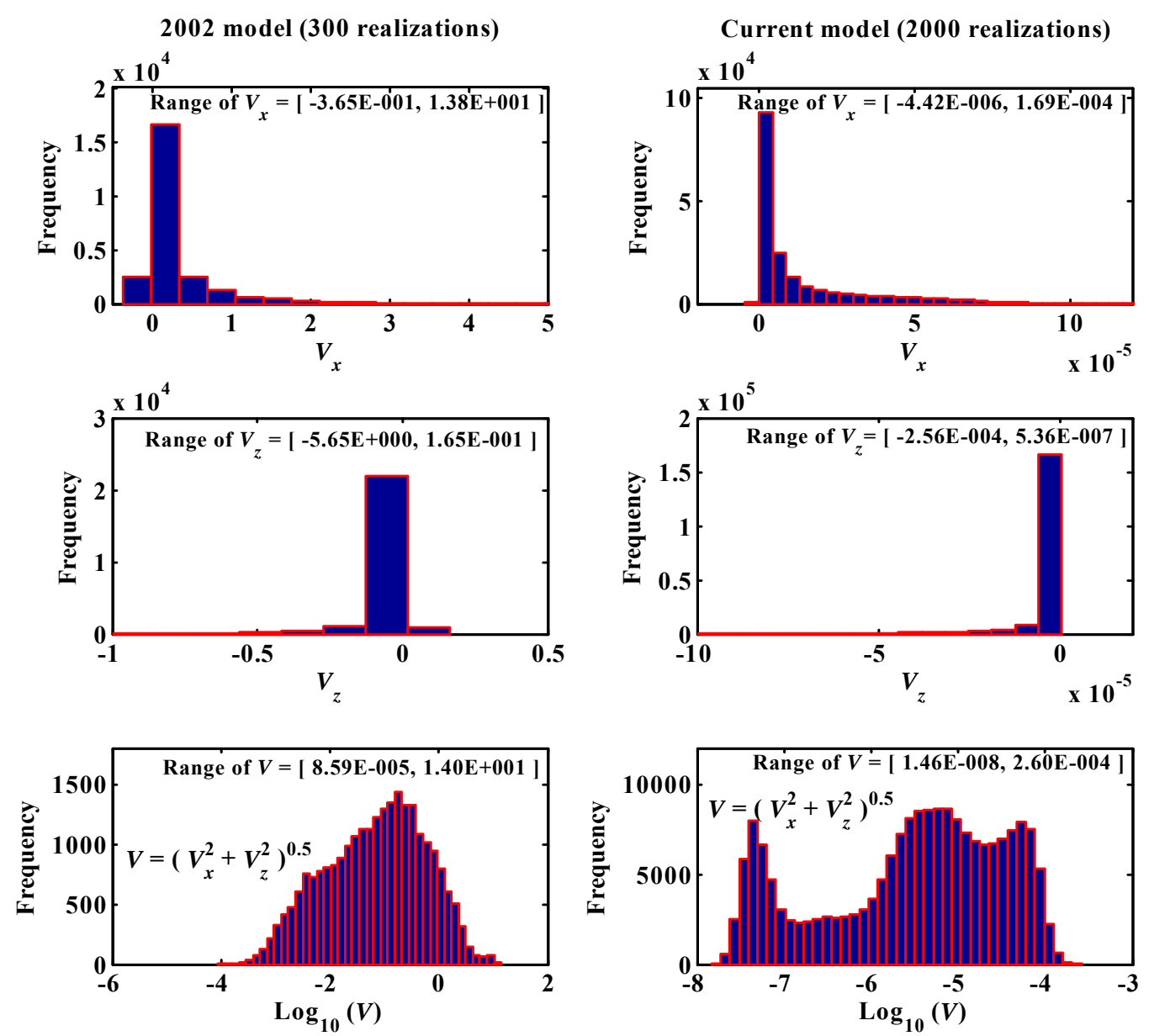

Figure 6.12. Distribution of Cannikin groundwater velocities at section C-C shown in Figure 6.10 and for all realizations. Horizontal velocity (a), vertical velocity (b), and magnitude of the velocity vector (c) are shown.

\section{SUMMARY OF MODEL VERIFICATION AND UNCERTAINTY REDUCTION}

The analysis presented in Sections 4 to 6 shows that the new data provided by CRESP did not contradict the conceptual framework or the parameter values used in the 2002 groundwater flow and transport models. The 2002 model is verified through a number of components. First, the high-resolution bathymetric data obtained from the oceanographic surveys (Johnson and Stewart, 2005) resulted in bathymetric profiles at the Cannikin and Long Shot models matching very closely the original profiles used in 2002. The bathymetric profiles constitute the upper boundary of the model domain, and the new bathymetric data verify the geometry of the model domain at Cannikin and Long Shot.

Second, the posterior distributions developed through the use of the Bayesian MCMC framework for the main parameters controlling the hydraulics of the island (i.e., recharge and hydraulic conductivity and their ratio) are encompassed within the original prior distributions used in the 2002 study. The posterior distributions conditioned on the new CRESP data and influenced the most by the MT-converted concentrations did not extend beyond the limits (or tails) of the original $K, R$, and $R / K$ distributions. This indicates the validity of the original 
distributions and that they were wide enough to account for all possibilities including the remote ones. This was the case for all of the three models.

Third, the revised flow solution using the distributions conditioned on the new data resulted in an ensemble mean (i.e., mean of Monte Carlo realizations) matching the head and chemistry data very well, with the exception of the chemistry data at Milrow. This ensemble mean was totally within the original model's mean \pm one standard deviation accounting for uncertainty. This is true for all sites at all well locations where head and/or chemistry data are available. More importantly, the data-constrained results show that the reduced uncertainty range (i.e., Monte Carlo mean \pm one standard deviation) is mostly within the 2002 uncertainty bounds expressed as mean \pm one standard deviation. This again confirms that the 2002 model accounted for a wide range of possibilities centered on the most likely scenario. When new data provided a better handle on parameter ranges, the wide uncertain range was trimmed from both sides, resulting in a new set of possible solutions encompassed within the original set of possible solutions.

Fourth, although the MT data indicate a much deeper transition zone at Milrow than that indicated by the chemistry data at well UAe-2, the possibility of a deeper transition zone was accounted for in the 2002 model by the wide range of recharge-conductivity ratio considered. Thus, despite building the distributions of $R$ and $K$ in the 2002 model around the calibrated values that gave a reasonable match to UAe- 2 chemistry and head data, the uncertainty built into these distributions did account for the possibility of a deeper (and shallower) transition zone than the data indicated.

Fifth, after integrating the new CRESP data, the head and chemistry data, and the prior knowledge about the island and its hydraulic parameters, the resulting groundwater fluxes had the same distribution as the original model, thereby verifying the original model. In fact, the groundwater flux distribution fits within the distribution predicted by the 2002 model. The latter distribution needs to be trimmed at the tails to yield the new distribution.

Sixth, using the porosity profiles developed through analysis of the MT data resulted in very slow flow velocities and no radionuclide mass flux breakthrough in a simulation time of 2,200 years. This provides another verification to the original model in the sense that the radionuclide mass flux standard deviation obtained in Hassan et al. (2002) was greater than the mean radionuclide mass flux (e.g., Figures 5.15, 5.16, 5.24, 5.25, 5.34, and 5.35). These 2002 results indicate that the mean minus one or two standard deviations results in a negative value, implying that the lower limit for radionuclide mass flux is essentially zero. This is now indicated by the new CRESP data, and it was included in the possibilities considered by Hassan et al. (2002).

Finally, when the porosity distribution of Hassan et al. (2002) is used with the groundwater fluxes generated from the new data-conditioned distributions, the resulting radionuclide mass fluxes lie within the uncertainty bounds of the 2002 model. The radionuclide mean mass flux plus one or two standard deviations is less than the same quantity in the 2002 model, providing a final verification for the 2002 model, exclusive of the impact of the new porosity data.

A dramatic reduction of uncertainty is achieved by conditioning on all available data sets. The parameter distributions covered a much narrower range than originally used in the 2002 study. This is very pronounced for the recharge-conductivity ratio, which is the 
determinant of the transition zone location. The MCMC presented in Section 4 indicates that for all sites, the percent reduction in parameter distribution variance is at least 70 percent. For the recharge-conductivity ratio, the percent reduction in the variance exceeded 85 percent for all sites.

The reduction of parameter uncertainty translated into similar reduction of uncertainty in the output of the groundwater flow models of the island. Salinity and head profiles at the analyzed well locations have a much narrower range of uncertainty than the 2002 study. The CRESP efforts also contributed to reducing the uncertainty in the fracture porosity, where the profiles provided by Unsworth et al. (2005) give a range of variability of less than an order of magnitude at the area of radionuclide transport (the cavity horizon and above). However, because these profiles moved the entire distribution toward much higher values than the 2002 study, the uncertainty in radionuclide mass flux discharging into the sea has been eliminated within the simulation time frame of 2,200 years. This resulted from the no-breakthrough output obtained for each of the 2,000 model realizations. If simulations are performed for a much longer timeframe, the radionuclide mass flux uncertainty is expected to be much smaller than in the 2002 model.

\section{SUMMARY AND CONCLUSIONS}

The modeling of Amchitka underground nuclear tests conducted in 2002 is verified and uncertainty in model input parameters, as well as predictions, has been reduced using newly collected data obtained by the summer 2004 field expedition of CRESP. Newly collected data that pertain to the groundwater model include magnetotelluric (MT) surveys conducted on the island to determine the subsurface salinity and porosity structure of the subsurface, and bathymetric surveys to determine the bathymetric maps of the areas offshore from the Long Shot and Cannikin Sites. Analysis and interpretation of the MT data yielded information on the location of the transition zone, and porosity profiles showing porosity values decaying with depth. These new data sets are used to verify the original model in terms of model parameters, model structure, and model output verification. In addition, by using the new data along with the existing data (chemistry and head data), the uncertainty in model input and output is decreased by conditioning on all the available data.

A Markov Chain Monte Carlo (MCMC) approach is adapted for developing new input parameter distributions conditioned on prior knowledge and new data. The MCMC approach is a form of Bayesian conditioning that is constructed in such a way that it produces samples of the model parameters that eventually converge to a stationary posterior distribution. The Bayesian MCMC approach enhances probabilistic assessment. Instead of simply propagating uncertainty forward from input parameters into model predictions (i.e., traditional Monte Carlo approach), MCMC propagates uncertainty backward from data onto parameters, and then forward from parameters into predictions.

Comparisons between new data and the original model, and conditioning on all available data using MCMC method, yield the following results and conclusions:

1) Model structure is verified at Long Shot and Cannikin where the high-resolution bathymetric data collected by CRESP yield profiles matching those used to construct the Long Shot and Cannikin model cross sections in 2002. 
2) Distributions of model input parameters (recharge, conductivity, and rechargeconductivity ratio) used in 2002 for the three sites are verified where the new data indicate distributions with narrower ranges (smaller uncertainty) but within the range employed in the 2002 model.

3) As a conservative approach, distribution of fracture porosity used in 2002 was deliberately skewed toward lower values. New CRESP data indicate that the selected porosity range was overly conservative. In addition, the range of porosity values obtained from the analysis of the MT data is found to generally be about three orders of magnitude lower than range of values used in the 2002 model, though the values themselves are much larger from the MT data.

4) Distributions of the flow model output (head distribution, salinity distribution, groundwater fluxes) resulting from the 2002 model for the three sites are verified where the new model output after conditioning on the data lie within the range of the 2002 model output.

5) Cannikin model output at location of well UAe-1 is not fully verified where the new model results for small salinity values are not fully enclosed by the uncertainty bounds of the original model output.

6) With the new porosities developed from the analysis of MT data, radionuclides require thousands of years to reach the seafloor. No breakthrough resulted for any of the three sites within the 2000 year model timeframe, despite ignoring all retardation mechanisms (sorption, radionuclide trapping in glass, matrix diffusion, and radioactive decay).

7) The no-breakthrough results verify the original model in the sense that this result lies within the uncertainty bounds of the 2002 model expressed as $<Q>+2 \sigma_{Q}$ and $<Q>$ $2 \sigma_{Q}$. The lower bound, $<Q>-2 \sigma_{Q}$, in the 2002 model gave negative values implying that the bound is essentially zero. The current results of no-breakthrough match this lower bound.

8) Significant uncertainty reduction is achieved for model input parameters (recharge, conductivity, and recharge-conductivity ratio) with the $R / K$ ratio experiencing a very dramatic reduction.

9) Uncertainty in groundwater fluxes is also reduced due to the reduction of $R / K$ uncertainty.

10) Groundwater velocities based on new data are orders of magnitude slower than the velocities produced by the 2002 model due to the higher porosity obtained from the analysis of the MT data.

11) Uncertainty reduction in radionuclide mass flux could not be assessed as the velocities are too small to produce radionuclide breakthrough within the model timeframe of 2,000 years. 


\section{REFERENCES}

Bates, B.C. and E.P. Campbell, 2001. A Markov chain Monte Carlo scheme for parameter estimation and inference in conceptual rainfall runoff modeling. Water Resources Research 37(4), 937-947.

Bear, J., 1972. Dynamics of Fluids in Porous Media. American Elsevier, New York.

Beetem, W.A., R.A. Young, C.L. Washington, and L.J. Schroder, 1971. Chemical analysis of water samples collected on Amchitka Island, Alaska. U.S. Geological Survey report USGS-474-135 (Amchitka-29), 23p.

Borg, I.Y., R. Stone, H.B. Levy and L.D. Ramspott, 1976. Information pertinent to the migration of radionuclides in ground water at the Nevada Test Site, Part 1: Review and analysis of existing information. Lawrence Livermore National Laboratory, Report UCRL-52078 Part 1, 216 p.

Campbell, E.P., D.R. Fox, and B.C. Bates, 1999. A Bayesian approach to parameter estimation and pooling in nonlinear flood event models. Water Resources Research 35(1), 211-220.

Claassen, H.C., 1978. Hydrologic processes and radionuclide distribution in a cavity and chimney produced by the Cannikin nuclear explosion, Amchitka Island, Alaska. U.S. Geological Survey, Professional Paper 712-D, 28p.

Cooley, R., 1982a. Incorporation of prior information on parameters into nonlinear regression groundwater flow models, 1, Theory. Water Resources Research 18: 965976.

Cooley, R., 1982b. Incorporation of prior information on parameters into nonlinear regression groundwater flow models, 2, Applications. Water Resources Research 19: 662-676.

CRESP (Consortium for Risk Evaluation with Stakeholder Participation), 2003. Amchitka Indendent Assessment Science Plan. http://www.cresp.org.

CRESP, (Consortium for Risk Evaluation with Stakeholder Participation), 2005. Amchitka Independent Science Assessment: Biological and Geophysical Aspects of Potential Radionuclide Exposure in the Amchitka Environment. http://www.cresp.org.

Diersch, J.J.G., 1998. Interactive, graphics-based finite-element simulation system FEFLOW for modeling groundwater flow contaminant mass and heat transport processes. FEFLOW Reference Manual, WASY Ltd., Berlin, 294 p.

Doherty, J., 1990. MODINV. Australian Center for Tropical Freshwater Resources, Townsville, Australia. 278 pp.

Doherty, J., 1994. PEST. Watermark Computing, Corinda, Australia. 122 pp.

Dudley, Jr.,W.W.,W.C. Ballance and V.M. Glanzman, 1977. Hydrology, in Merritt, M.L. and R.G. Fuller (eds.). The environment of Amchitka Island, Alaska. Energy Research and Development Administration, Technical Information Center, pp.35-51p. 
Essington, E.H., P.R. Fenske, and W.E. Nork, 1970. Radioactivity in water, Project Milrow. Teledyne Isotopes report for the U.S. Atomic Energy Commission, Nevada Operations Office, NVO-1229-135, 49p.

Fenske, P., 1972. Hydrology and radionuclide transport, Amchitka Island, Alaska. Technical Report Series H-W Hydrology and Water Resources Publication No. 12, Desert Research Institute.

Forsstrom, A. and D. Barnes, 2005. Characterization of Amchitka Island Subsurface: Groundwater Modeling in the Vicinity of the Long Shot Test Shot. In Powers, C.W., J. Burger, D. Kosson, M. Gochfeld, and D. Barnes (eds.), 2005, Amchitka Independent Science Assessment: Biological and Geophysical Aspects of Potential Radionuclide Exposure in the Amchitka Environment. Consortium for Risk Evaluation with Stakeholder Participation, www.cresp.org.

Freer, J., K.J. Beven and B. Ambroise, 1996. Bayesian estimation of uncertainty in runoff prediction and the value of data: an application of the GLUE approach. Water Resources Research 32(7):2161-2173.

Hassan, A., K. Pohlmann and J. Chapman, 2002. Modeling Groundwater Flow and Transport of Radionuclides at Amchitka Island's Underground Nuclear Tests: Milrow, Long Shot, and Cannikin. Desert Research Institute, Division of Hydrologic Sciences, Publication No.45172, DOE/NV/11508--51.

Haario, H., E. Saksman, and J. Tamminem, 2001. An adaptive Metropolis algorithm. Bernoulli, 7(2), 223-242.

Hastings, W.K., 1970. Monte Carlo sampling methods using Markov chains and their applications. Biometrika, 57, 97-109.

Hill, M.C., 1992. A computer program (MODFLOWP) for estimating parameters of a transient, three-dimensional groundwater flow model using nonlinear regression. USGS OFR 91-484. $358 \mathrm{pp}$.

Hoeksema, R.J. and P.K. Kitanidis, 1984. An application of the geostatistical approach to the inverse problem in two-dimensional groundwater modeling. Water Resources Research 20, no. 7: 1003-1020.

Holmes and Narver, Inc., 1976. Amchitka Island Map Atlas. U.S. Atomic Energy Commission, Nevada Operations Office, Archive and Records Center, 41 sheets.

International Atomic Energy Agency (IAEA), 1998. The Radiological Situation at the Atolls of Mururoa and Fangataufa, Volume 4, Releases to the Biosphere of Radionuclides from Underground Nuclear Weapons Tests at the Atolls. IAEA, Austria, 270 p.

Johnson, M. and C. Stewart, 2005. Geophysical Investigations I - Oceanographic Investigations of Bathymetry, Ocean Floor Discharge of Freshwater through the Ocean Floor, and Sediment Distribution. In Powers, C.W., J. Burger, D. Kosson, M. Gochfeld, and D. Barnes (eds.), 2005, Amchitka Independent Science Assessment: Biological and Geophysical Aspects of Potential Radionuclide Exposure in the Amchitka Environment. Consortium for Risk Evaluation with Stakeholder Participation, www.cresp.org. 
Kinzelbach, W., 1988. The random walk method in pollutant transport simulation. In Groundwater Flow and Quality Modeling. E. Custodio, A. Gurgui, J.P. Lobo Ferreira, D. Reidel (eds.), Norwell, Mass, 227-246.

Kuczera, G., 1983. Improved parameter inference in catchment models: 1. Evaluating parameter uncertainty. Water Resources Research 19(5), 1151-1162.

Kuczera, G. and M. Mroczkowski, 1998. Assessment of hydrologic parameter uncertainty and the worth of multiresponse data, Water Resources Research, 34(6), 1481-1489.

Kuczera, G. and E. Parent., 1998. Monte Carlo assessment of parameter uncertainty in conceptual catchment models: The Metropolis algorithm. Journal of Hydrology 211, 6985 .

LaVenue, A.M., B.S. RamaRao, G. Marsily, and M.G. Marietta, 1995. Pilot point methodology for automated calibration of an ensemble of conditionally simulated transmissivity fields, 2, Application. Water Resources Research 31, no. 3: 495-516.

Lee,W.H., 1969a. Some physical properties of rocks in drill hole UAe-2, Amchitka Island, Alaska. U.S. Geological Survey, Report USGS-474-5, 12p.

Lee,W.H., 1969b. Some physical properties of rocks in drill hole UAe-1, Amchitka Island, Alaska. U.S. Geological Survey, Report USGS-474-48, 13p.

Lee,W.H., 1969c. Some physical properties of rocks in drill hole UAe-3, Amchitka Island, Alaska. U.S. Geological Survey, Report USGS-474-6, 11 p.

Lee,W.H., 1969d. Some physical properties of rocks in drill hole UAe-6c, Amchitka Island, Alaska. U.S. Geological Survey, Report USGS-474-33, 13p.

Marshall, L., D. Nott and A. Sharma, 2004. A comparative study of Markov chain Monte Carlo methods for conceptual rainfall-runoff modeling. Water Resources Research 40, W02501, doi:10.1029/2003WR002378.

Metropolis, N., A.W. Rosenbluth, M.N. Rosenbluth, A.H. Teller, and E. Teller, 1953. Equations of state calculations by fast computing machines. J. Chem. Phys., 21, 10871092.

Nork, W.E., J.V.A. Sharp and C.L. Carnahan, 1965. Ground-water safety feasibility project Long Shot. Hazleton-Nuclear Science Corporation, Palo Alto, California, 49p.

Pawloski, G.A., A.F.B. Tompson, and S.F. Carle, 2001. Evaluation of the Hydrologic Source Term from Underground Nuclear Tests on Pahute Mesa at the Nevada Test Site: The Cheshire Test. Lawrence Livermore National Laboratory, UCRL-ID-147023, variable paging.

Poeter, E.P. and M.C. Hill. 1997. Inverse models: A necessary next step in groundwater modeling. Ground Water 35(2): 250-260.

RamaRoa, B.S., M.A. LaVenue, G. Marsily, and M.G. Marietta, 1995. Pilot point methodology for automated calibration of an ensemble of conditionally simulated transmissivity fields, 1, Theory and computational experiments. Water Resources Research 31, no. 3: 475-493. 
Smith, A.F.M. and G.O. Roberts, 1993. Bayesian computation via the Gibbs sampler and related Markov chain Monte Carlo methods. J. R. Stat. Soc., Ser. B, 55, 3-23.

Snow, D.T., 1968. Rock fracture spacings, openings and porosities. Journal Soil Mech. Found. Div., Proc. Amer. Soc. Civil Engineers, 94, pp.73-91.

Tierney, L., 1994. Markov chains for exploring posterior distributions (with discussion), Ann. Stat., 22, 1701-1762.

Tompson, A.F.B. and L.W. Gelhar, 1990. Numerical simulation of solute transport in threedimensional randomly heterogeneous porous media. Water Resources Research, 26(10):2541-2562.

Tompson, A.F.B., C.J. Bruton and G.A. Pawloski (eds.), 1999. Evaluation of the Hydrologic Source Term From Underground Nuclear Tests in Frenchman Flat at the Nevada Test Site: The Cambric Test. Lawrence Livermore National Laboratory report UCRL-ID132300, 319p.

Unsworth, M., W. Soyer and V. Tuncer, 2005. Magnetotelluric measurements for determining the subsurface salinity and porosity structure of Amchitka Island, Alaska. In Biological and Geophysical Aspects of Potential Radionuclide Exposure in the Amchitka Marine Environment, edited by Powers et al. Consortium for Risk Evaluation with Stakeholder Participation, Institute for Responsible Management, Piscataway, New Jersey.

U.S. Army Corps of Engineers and U.S. Geological Survey, 1965. Project Long Shot Amchitka Island, Alaska, Geologic and Hydrologic Investigations (Phase I). Unnumbered report.

U.S. Department of Energy (DOE), 2000. United States Nuclear Tests, July 1945 through September 1992. Nevada Operations Office report DOE/NV--209-Rev 15, 162 p.

von Huene, R., W.J. Carr, D. McManus and M. Holmes, 1971. Marine geophysical study around Amchitka Island, Western Aleutian Islands, Alaska. U.S. Geological Survey, Report USGS-474-74 (Amchitka-22), 31p.

Weir, I.S., 1997. Fully Bayesian reconstructions from single-photon emission computed tomography data. JASA J. Am. Stat. Assoc., 92, 49-60.

Xiang, Y., J.F. Sykes and N.R. Thomson, 1992. A composite L1 parameter estimator for model fitting in groundwater flow and solute transport simulation. Water Resources Research 29, no. 6: 1661-1673.

Zimmerman, D.A., G. de Marsily, C.A. Gotway, M.G. Marietta, C.L. Axness, R.L. Bras, J. Carrera, G. Dagan, P.B. Davies, D.P. Gallegos, A. Galli, J. Gómez-Hernández, P. Grindgrod, A.L. Gutjahr, P.K. Kitanidis, A.M. LaVenue, D. McLaughlin, S.P. Neuman, B.S. RamaRao, C. Ravenne and Y. Rubin, 1998. A comparison of seven geostatistically based inverse approaches to estimate transmissivities for modeling advective transport by groundwater flow. Water Resources Research 34(6): 1373-1413. 
THIS PAGE INTENTIONALLY LEFT BLANK 


\section{APPENDIX: MCMC SENSITIVITY STUDIES USING MILROW MODEL}

Sensitivity runs are conducted to gain a better understanding of the impact of different data sets on the results of MCMC conditioning and the impact of combinations of these data sets on the resulting posterior distributions. As discussed in Section 4.7, two issues are addressed and studied in this appendix. The first is how to use the MT-interpreted transition zone location and convert it such that it is equivalent to the model output, i.e., salinity. The second issue is how the different data sets (head, salinity, and MT data) can be combined.

In regards to the first issue, there are four options for converting the MT data to salinity data. The first option is to use the top and bottom of the transition zone identified by Unsworth et al. (2005) based on the geophysical inversion of the MT data (i.e., Lines A and $\mathrm{B}$ in Figure 4.17, which is reproduced here as Figure A.1 for completeness) as two lateral lines of freshwater and saltwater concentrations, respectively. This was shown in Figure 4.18a. In this case, the model's cells or elements that are crossed by Line A are assigned a salinity value equal to zero (freshwater concentration), and those that are crossed by Line B are assigned a saltwater concentration value, $C_{\max }=18,100 \mathrm{mg} / \mathrm{L}$ chloride concentration.

Figure A. 2 displays the MCMC conditioning results using the MT-converted concentrations with this first option. In the figure, $P(R), P(K)$, and $P(R / K)$ denote the prior distributions which are taken to be the same as the distributions used in the 2002 model. $P\left(R D_{1}\right), P\left(K \mid D_{1}\right)$, and $P\left(R / K \mid D_{1}\right)$ denote the posterior distributions that are obtained by conditioning on the MT-converted concentrations. Therefore, $D_{1}$ in Figure A.2 denotes the MT-converted concentration using the first option mentioned above.

It can be seen from the figure that the use of the MT data as two lateral lines of freshwater and saltwater concentrations yields a significant increase in the recharge values, where the recharge posterior is shifted toward higher values with $\mu_{R}$ increasing from 2.08 $\mathrm{cm} / \mathrm{yr}$ to 3.04 after conditioning. The mode of the recharge distribution has increased from 1.2 to about $2.4 \mathrm{~cm} / \mathrm{yr}$. A slight reduction in uncertainty is achieved where $\sigma_{R}$ has decreased from 1.38 to $1.23 \mathrm{~cm} / \mathrm{yr}$. The impact of conditioning is significant for the conductivity. The long tail in the prior distribution has been trimmed by conditioning on the MT data represented as two lateral lines. Both the mean and mode of the distribution are lower in the posterior than in the prior. The posterior density peak is more than double the prior peak. The spreading of the distribution represented by $\sigma_{K}$ has decreased about 63 percent relative to the prior.

The MCMC conditioning impact on $R / K$ is more dramatic than the impacts on $R$ and $K$. First, the distribution changed from lognormal in the prior to normal in the posterior. Second, the mean of the distribution more than doubled after conditioning and the mode increased from 79 in the prior to about 320 in the posterior. Spreading is reduced about 55 percent compared to the prior, and the peak density is increased about 38 percent after conditioning.

By conditioning on the MT as two lateral lines, realizations with deep transition zone match the data well, as the top line of zero concentrations (freshwater) is matched by these realizations and the bottom line is also matched. The distance occupied by the transition zone 
in the model output is roughly $600 \mathrm{~m}$, and the distance between the upper and lower bounds of the transition zone from the MT data is about $800 \mathrm{~m}$. Therefore, once a transition zone in a realization starts below Line A in Figure A.1 (i.e., matching the freshwater concentration line), it is going to match Line $\mathrm{B}$, as the modeled transition zone will end before Line $\mathrm{B}$. This is the reason for the shift in the $R / K$ posterior to higher $R / K$ values with the mode being at 320 .

It is important to note here that these results and the values discussed and shown in Figure A. 2 and subsequent figures (i.e., $\mu, \sigma$, and distribution mode) should be taken as qualitative measures since the distributions plotted in Figure A.2 and subsequent figures are just fits to the posterior samples obtained from the MCMC algorithm. In some cases, the fits are very good and in others they are not as good.

The second option of converting the MT data to concentrations is to fill all the domain elements above line A (Figure A.1) with freshwater concentration and all the elements below line B with seawater concentration. This was shown in Figure 4.18b. The results of using this option as the conditioning data in MCMC are displayed in Figure A.3. The MCMC conditioning results for the recharge indicate a shift in the distribution toward higher recharge values with the distribution mean increasing from 2.08 to $3.32 \mathrm{~cm} / \mathrm{yr}$ and the mode increasing from 1.2 to $2.5 \mathrm{~cm} / \mathrm{yr}$. A small increase in the distribution spread occurred, where the standard deviation, $\sigma_{R}$, increased from 1.38 to $1.5 \mathrm{~cm} / \mathrm{yr}$, and the peak density decreased. For the conductivity, the posterior distribution shifted to lower values and significant spreading reduction occurred, where $\sigma_{K}$ decreased from 0.0104 to $0.0045 \mathrm{~m} / \mathrm{d}$ and the density peak doubled. The distribution mode slightly shifted to a lower $K$ value.

The impact of conditioning on $R / K$ is shown in Figure A.3c. The distribution shifted to higher ratios and the mode changed from a value of 79 in the prior distribution to a value of 320 in the posterior. The peak density did not change and spreading decreased about 40 percent. The MT data indicate a deeper transition zone than the collective prior knowledge, which results in a shift of the distribution mode from an $R / K$ value of 79 to a value of 320 . The $R / K$ posterior is very close in shape to a normal distribution, whereas the prior was lognormally distributed.

The third option of converting MT data involves assigning the model elements that are crossed by Line A freshwater concentration and assigning seawater concentration to the elements crossed by Line B and all the elements below it. This was shown in Figure 4.19a. The results shown in Figure A.4 show that this option yields posterior distributions similar to a large degree to the first option discussed above. A significant increase in the recharge values occurred after conditioning, where the recharge posterior is shifted toward higher values with $\mu_{R}$ increasing from 2.08 to $3.31 \mathrm{~cm} / \mathrm{yr}$. The mode of the recharge distribution has increased from 1.2 to about $2.6 \mathrm{~cm} / \mathrm{yr}$. Uncertainty in the recharge distribution has not changed after conditioning. The impact of conditioning is significant for the conductivity. The long tail in the prior distribution has been trimmed by conditioning on the MT data, represented as two lateral lines. Both the mean and mode of the distribution are lower in the posterior than in the prior. The posterior density peak is more than double the prior peak. The spreading of the distribution represented by $\sigma_{K}$ has decreased about 64 percent relative to the prior. 
The MCMC conditioning impact on $R / K$ is more dramatic than the impacts on $R$ and $K$. The $R / K$ distribution changed from lognormal in the prior to normal in the posterior. However, this may be a result of a better fit (not a perfect one) to the data using a normal distribution as opposed to a lognormal one. Second, the mean of the distribution more than doubled after conditioning and the mode increased from 79 in the prior to about 375 in the posterior. Spreading is reduced about 72 percent compared to the prior, and the peak density is about doubled after conditioning.

The last option is the reverse of the third option, where the elements crossed by or above line A (Figure A.1) are assigned freshwater concentration and the only elements crossed by line B are assigned seawater concentration. The results of using this option for MCMC conditioning are displayed in Figure A.5. Similar to the previous options, the recharge posterior is located at higher $R$ values than the prior. The distribution mean increased from 2.08 to $3.52 \mathrm{~cm} / \mathrm{yr}$ and the mode shifted from a value of 1.2 to a value of about $2.7 \mathrm{~cm} / \mathrm{yr}$. The distribution spread increased and the peak density decreased.

The hydraulic conductivity posterior shifted to lower $K$ values than the prior. The high-K tail of the prior distribution is trimmed after conditioning and $\sigma_{K}$ decreased from 0.0104 to $0.0041 \mathrm{~m} / \mathrm{d}$. The peak density more than doubled after conditioning and the mode moved from 0.01 to about $0.007 \mathrm{~m} / \mathrm{d}$. The shape and location of the conductivity posterior is very similar to the first option (Figure A.2b), second option (Figure A.5b), and third option (Figure A.4b).

Recharge-conductivity posterior shifted to higher ratios and the distribution mean increased with a factor greater than 2 . The mode also shifted to a ratio of about 320 compared to the 79 value of the prior. Unlike the previous options, this option produces an $R / K$ posterior with high spread. The posterior distribution standard deviation, $\sigma_{R / K}$, is about 132 in this fourth option, whereas it was 46, 64, and 28 in the previous three options, respectively.

It can be seen from the previous analysis that the MT data yield an $R / K$ posterior with much higher $R / K$ values than the prior. The mode of the posterior distribution in all options is located between 300 and 400, whereas the prior mode is at 79. Since all options yield a similar $R / K$ posterior, the third option is selected for the main analysis presented in Section 4.7. This option honors both the MT and salinity data at UAe-2 below Line B (Figure A.1), and the top boundary of the transition zone (Line A in Figure A.1) is also honored.

The second issue studied here pertains to the different ways to combine the three data sets available: MT-converted concentration, salinity, and head data. With these three data sets, there are many different ways to use the data in the MCMC algorithm. Four approaches are evaluated here:

1) One data set is used at a time to obtain the posterior distribution; in this approach, three posterior distributions are obtained, one for each data set conditioning.

2) The three data sets are combined and used at the same time with equal weights.

3) One data set is used first to obtain the posterior distribution $t$ hen this posterior is assumed to be a prior for use with the second data set to develop a new posterior, which in turn is used as a prior for use with the third data set to develop the final posterior. This approach is denoted here as the sequential conditioning approach. 
4) The salinity data set and MT-converted concentration data set are combined as a single data set and used to develop a posterior distribution for the model parameters, which is then used as a prior with the head data to develop the final posterior.

\section{Conditioning on Individual Data Sets}

Figure A.6 presents the results of conditioning on the head data at the UAe-2 location at Milrow. The head data indicate a deeper transition zone than what the prior distribution implies. As a result, the recharge posterior distribution is shifted toward higher values and the conductivity posterior distribution is shifted to lower values. Some uncertainty reduction occurs for the recharge and more significant reduction is achieved for the conductivity. The mode of the recharge distribution increased from 1.2 to $1.7 \mathrm{~cm} /$ year after conditioning on the heads, whereas the mode of the conductivity distribution slightly decreased from 0.011 to $0.01 \mathrm{~m} / \mathrm{d}$.

The posterior distribution of the recharge-conductivity ratio shifted toward higher values, with the distribution mode increasing from 79 to a value of about 130 . The peak density slightly dropped (about 10\%) after conditioning on the head data.

The salinity data from UAe-2 is used next as the only conditioning data. The results of MCMC analysis for this case are shown in Figure A.7. Compared to the head data from the same well, the impact of salinity data on the MCMC results is smaller. This is probably due to the fact that the prior distributions for $R$ and $K$ used here are the parameter distributions used in the 2002 models. These distributions were qualitatively selected based on the transition zone location indicated by the salinity data at UAe-2. Thus, the prior distribution honored this data set to some extent. When using this data set to condition the distribution, small changes occur to the distribution.

The recharge posterior has more or less the same mean as the prior, but the spreading has decreased about 20 percent ( $\sigma_{R}$ has decreased from 1.379 to 1.08 ). The mode of the posterior is slightly higher than the mode of the prior. The peak density of the posterior is about 12 percent higher than that of the prior. The impact of conditioning on salinity data on the hydraulic conductivity is very similar to the impact on recharge. The same salient features are observed regarding the conductivity posterior.

The impact on $R / K$ is also small compared to the head impact shown in Figure A.6. The shape and pattern of the $R / K$ posterior and its location relative to the prior are very similar to the recharge and conductivity results shown in Figure A.7a, b. The mean of $R / K$ posterior has slightly decreased and the spreading has about 17 percent reduction. The posterior distribution mode is at a recharge-conductivity ratio of about 90 compared to the prior value of about 79 .

The last data set used as an individual set is the MT-converted concentration data obtained using the third option discussed above. The MCMC conditioning on this data set was shown in Figure A.4 and discussed above.

Comparing Figures A.4, A.6, and A.7 shows that the salinity data require the shallowest transition zone (lower $R / K$ values) and the MT data converted with the third option requires the deepest transition zone. The head data require a transition zone in between these two cases. This is evident from comparing the $R / K$ posterior in the three 
figures. For example, the posterior mode is about 90 in the salinity conditioning case (Figure A.7), about 130 in the head conditioning case (Figure A.6), and about 375 in the MT conditioning case (Figure A.4). The peak density (the density of the posterior corresponding to the mode) is highest and spreading lowest for the MT case. The density peak is lowest and the spreading is highest in the head conditioning case.

\section{Conditioning on All Data Sets Combined}

The three data sets are combined as a single data set and used for MCMC conditioning. In this case, the two terms required for the likelihood function, the sum of squared errors $\sum_{j=1}^{N_{D}}\left[\mathbf{D}\left(\mathbf{x}_{j}\right)-M\left(\mathbf{G}, \mathbf{x}_{j} ; \Theta\right)\right]^{2}$, and the error variance $\sigma_{\varepsilon}^{2}$ are obtained as weighted averages of the individual terms for each of the three data sets.

The results of this case are shown in Figure A.8. The recharge posterior has higher mean and mode than the prior. The mean of the posterior is 2.46 , whereas the prior mean is $2.08 \mathrm{~cm} / \mathrm{yr}$. The mode has shifted from about 1.2 to $2.0 \mathrm{~cm} / \mathrm{yr}$ after conditioning on all data sets combined. Spreading has decreased a little, where $\sigma_{R}$ has decreased from 1.379 to 1.084 $\mathrm{cm} / \mathrm{yr}$. The mode of the hydraulic conductivity has not changed much but the corresponding peak has increased about 60 percent. Accordingly, the spreading has dramatically decreased (about 50\%). The long tail of the distribution at higher conductivity values is trimmed after conditioning on all data sets.

The impact of all data combined is dramatic for $R / K$. The distribution changed from a lognormal to a normal one. The mode has shifted from a value of about 79 in the prior to a value of about 190 in the posterior. The standard deviation of the $\mathrm{R} / \mathrm{K}$ posterior distribution is about one third that of the prior, indicating dramatic reduction of uncertainty. It is interesting to note that the peak posterior density occurs at a value of 190 , which corresponds, or is very close, to the average of the posterior modes in the individual cases, which were 90 for the salinity case, 130 for the head case, and 375 for the MT case.

\section{Sequential Conditioning Approach}

In this approach, the three data sets are used sequentially and each posterior is used as a prior for the subsequent data set conditioning. One data set is used first to obtain the posterior distribution, then this posterior is assumed to be a prior for use with the second data set to develop a new posterior, which in turn is used as a prior for use with the third data set to develop the final posterior. Six different combinations exist with the three data sets available. These are summarized in Table A.1.

Table A.1. Different sequential combinations of using the three data sets in MCMC conditioning 


\begin{tabular}{|c|ccc|}
\hline Case \# & First Data Set, $D_{1}$ & Second Data Set, $D_{2}$ & Third Data Set, $D_{3}$ \\
\hline 1 & heads (UAe-2) & salinities (UAe-2) & MT (third option) \\
2 & heads (UAe-2) & MT (third option) & salinities (UAe-2) \\
3 & salinities (UAe-2) & heads (UAe-2) & MT (third option) \\
4 & salinities (UAe-2) & MT (third option) & heads (UAe-2) \\
5 & MT (third option) & salinities (UAe-2) & heads (UAe-2) \\
6 & MT (third option) & heads (UAe-2) & salinities (UAe-2) \\
\hline
\end{tabular}

The results of these six cases are displayed in Figures A.9 through A.14. The figures are self-explanatory and it does not add much to discuss them in detail like the previous figures. However, the salient features in the figures are discussed here. The most important finding is that the final posterior depends largely on the last data set used for conditioning. It can be seen from the figures that when the MT is the last data set used for conditioning (Cases 1 and 3), the final posteriors look similar to each other and to the case where only MT is used for conditioning (Figure A.4) regardless of the previous conditioning sequence. These posterior distributions are similar in terms of the shape, location, model, mean, and standard deviation, especially for $R / K$. When the heads are used as the last data set for MCMC conditioning (Cases 4 and 5), the final posteriors are similar (but not as much as for the MT case) to each other. However, the posteriors for Cases 4 and 5 are somewhat different from the case of using only these heads for conditioning (Figure A.6).

The use of the salinity data as the last conditioning data set yields different posteriors (Cases 2 and 6). Furthermore, these posteriors are different from those obtained by conditioning on the salinity data only (Figure A.5). These results show one of the main features of the Bayesian framework used; when the conditioning data set is large (e.g., the MT Cases 1 and 3), the prior distribution does not significantly impact the result. Conversely, when the data set is limited in size (e.g., the head and salinity Cases 2, 4, 5, and 6), the prior distribution affects the MCMC results.

\section{Combining Transition-zone-related Data}

The fourth approach is to combine the salinity data set and MT-converted concentration data set (transition-zone-related data) as a single data set and use it to develop a posterior distribution for the model parameters. This posterior is then used as a prior with the head data to develop the final posterior. This approach is adapted in the analysis discussed in Section 4.7. By conditioning on the combined transition-zone-related data sets (i.e., salinities and MT-converted concentrations) and subsequently conditioning on heads, we honor the fact that head data are subject to the least amount of subjectivity or interpretations. Conditioning on the head last is considered the best approach because the salinity and MT will have more data than the head, and thus it would be a way of not losing the impact of the head data. 


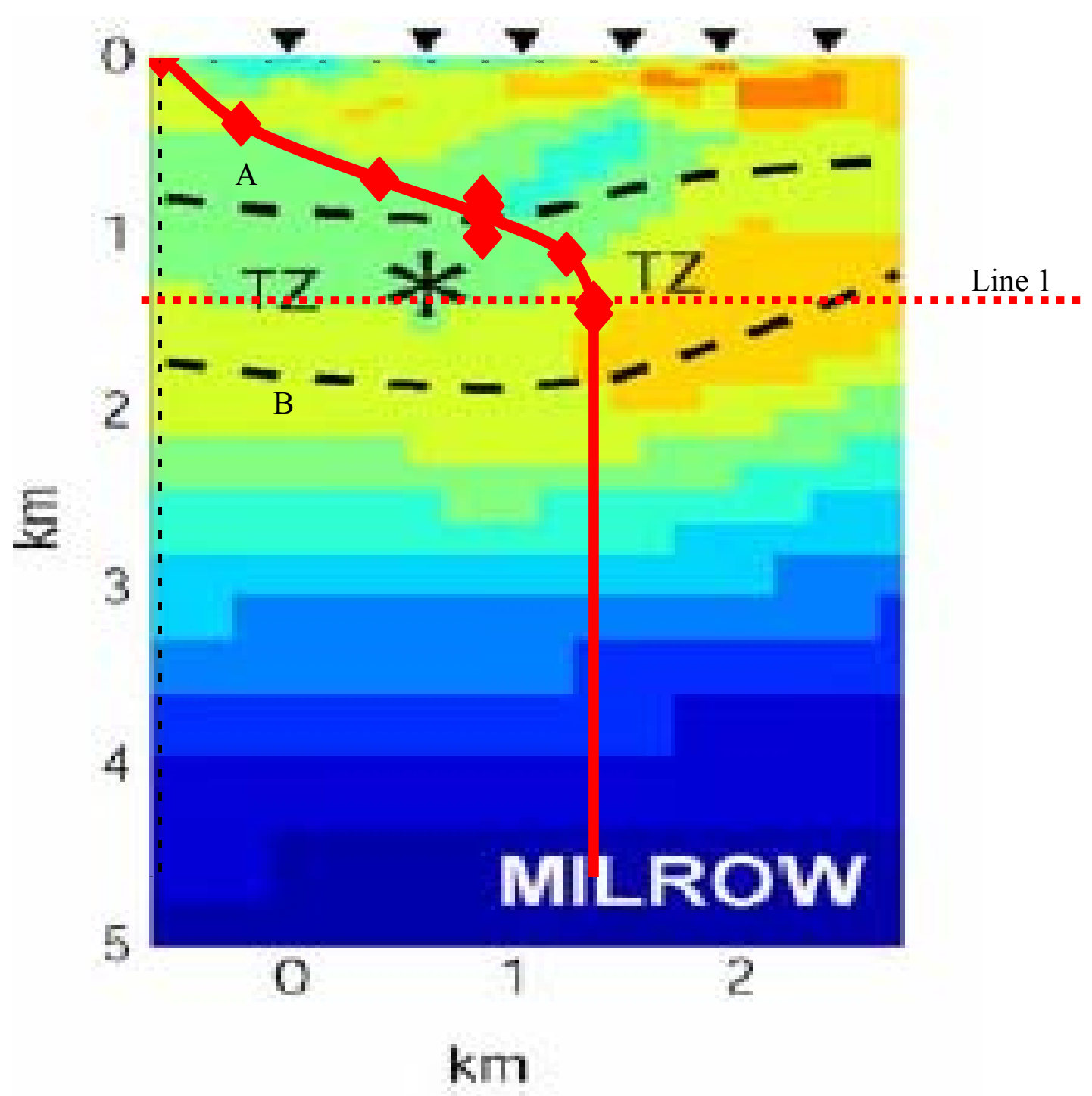

Figure A.1. Resistivity map and interpreted transition zone (TZ) boundaries (Lines A and B) from Unsworth et al. (2005) with the salinity profile from UAe-2 at Milrow (red line) superimposed. Line 1 indicates the beginning of seawater per the salinity data. 

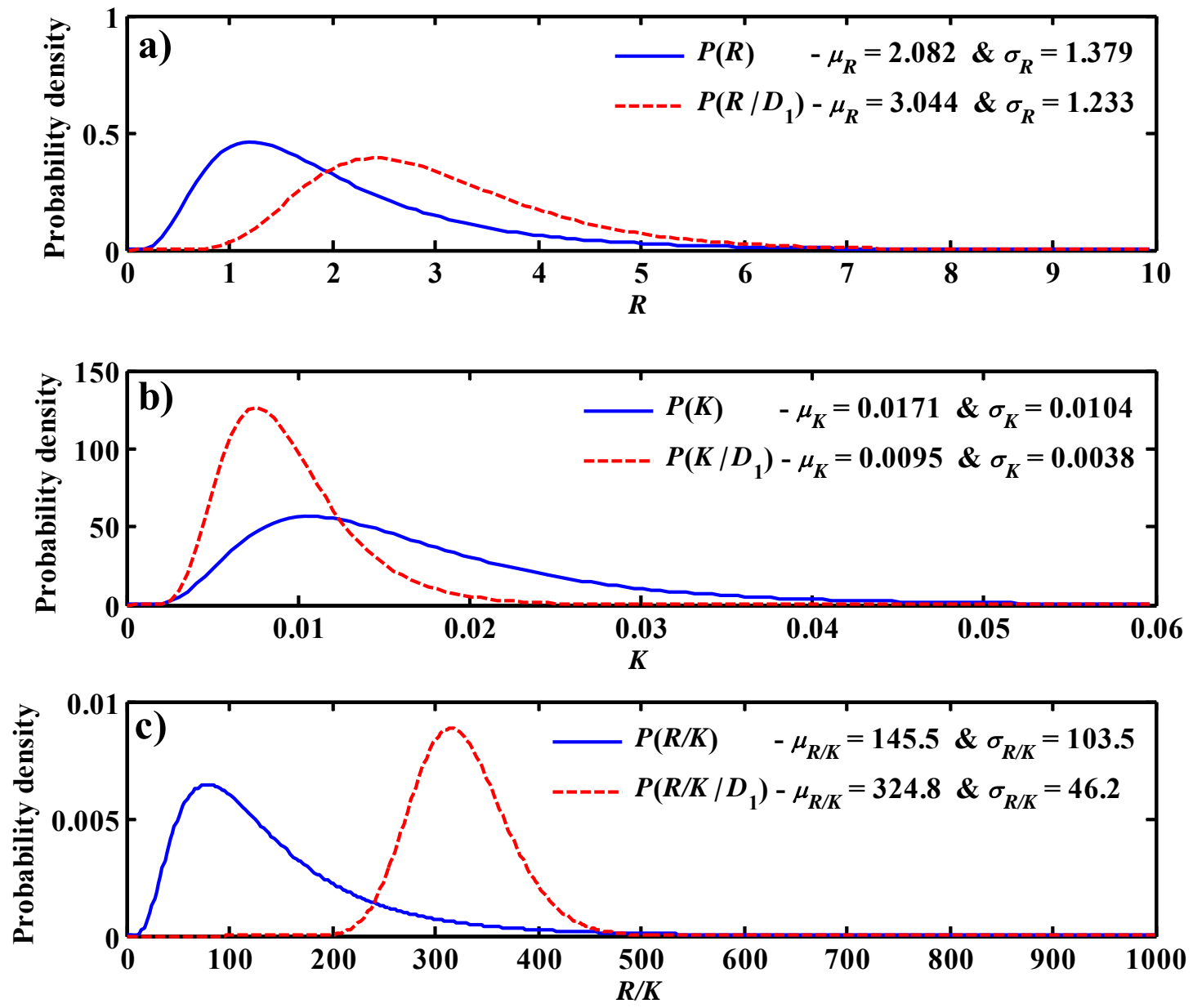

Figure A.2. Results of the MCMC conditioning approach for Milrow, where $D_{1}$ indicates the MT-converted concentrations using the first option. 

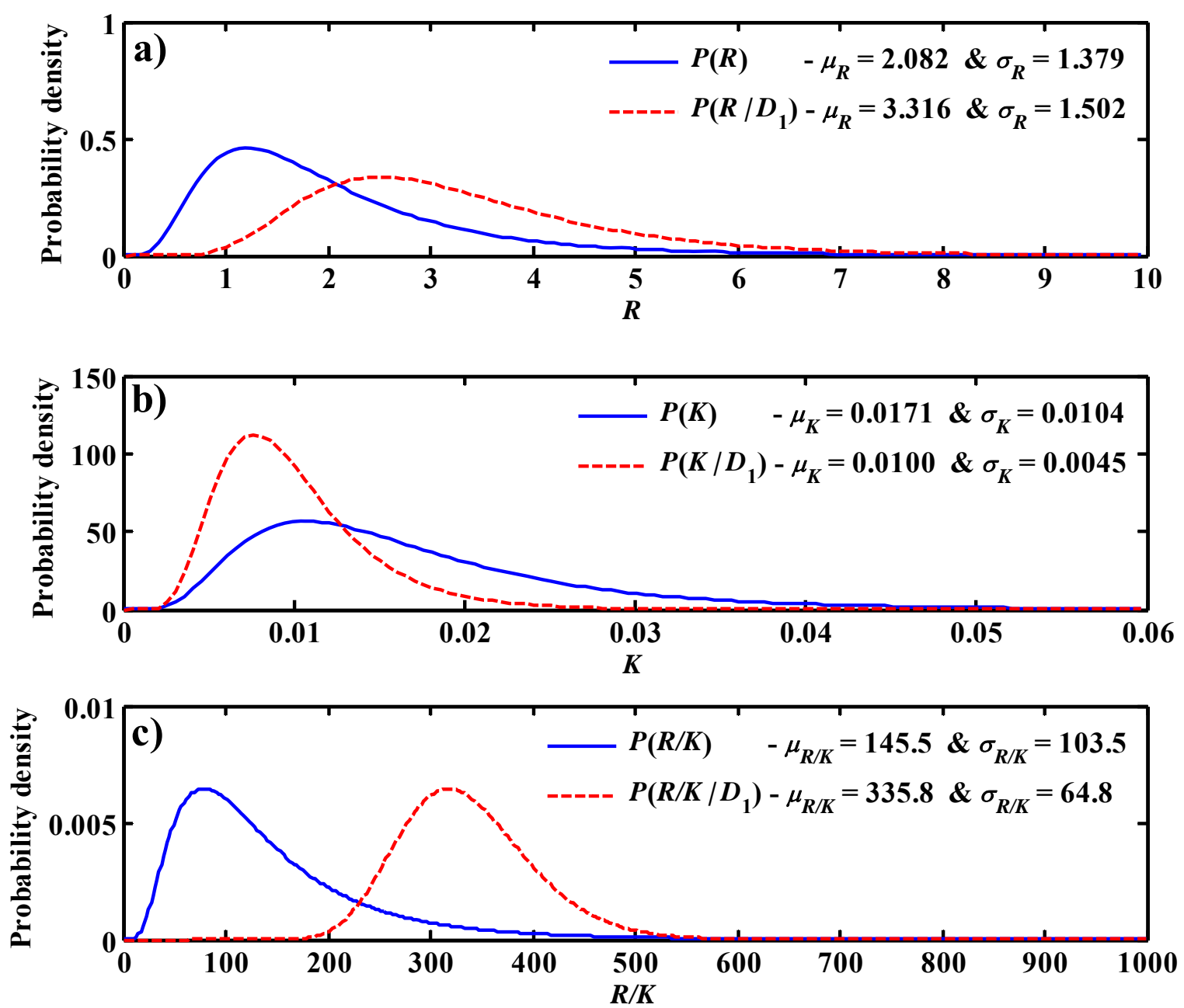

Figure A.3. Results of the MCMC conditioning approach for Milrow, where $D_{1}$ indicates the MT-converted concentrations using the second option. 

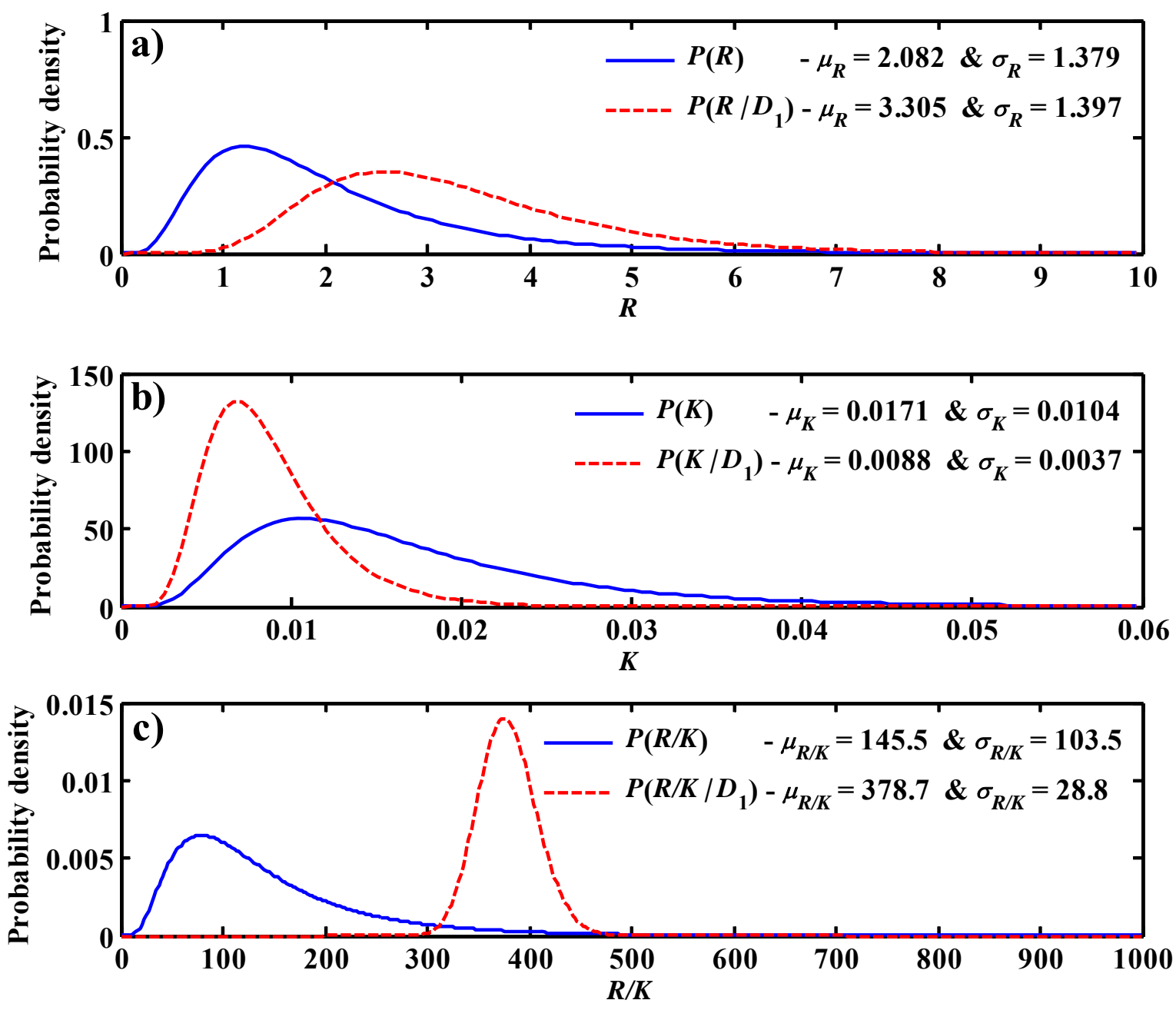

Figure A.4. Results of the MCMC conditioning approach for Milrow, where $D_{1}$ indicates the MT-converted concentrations using the third option. 

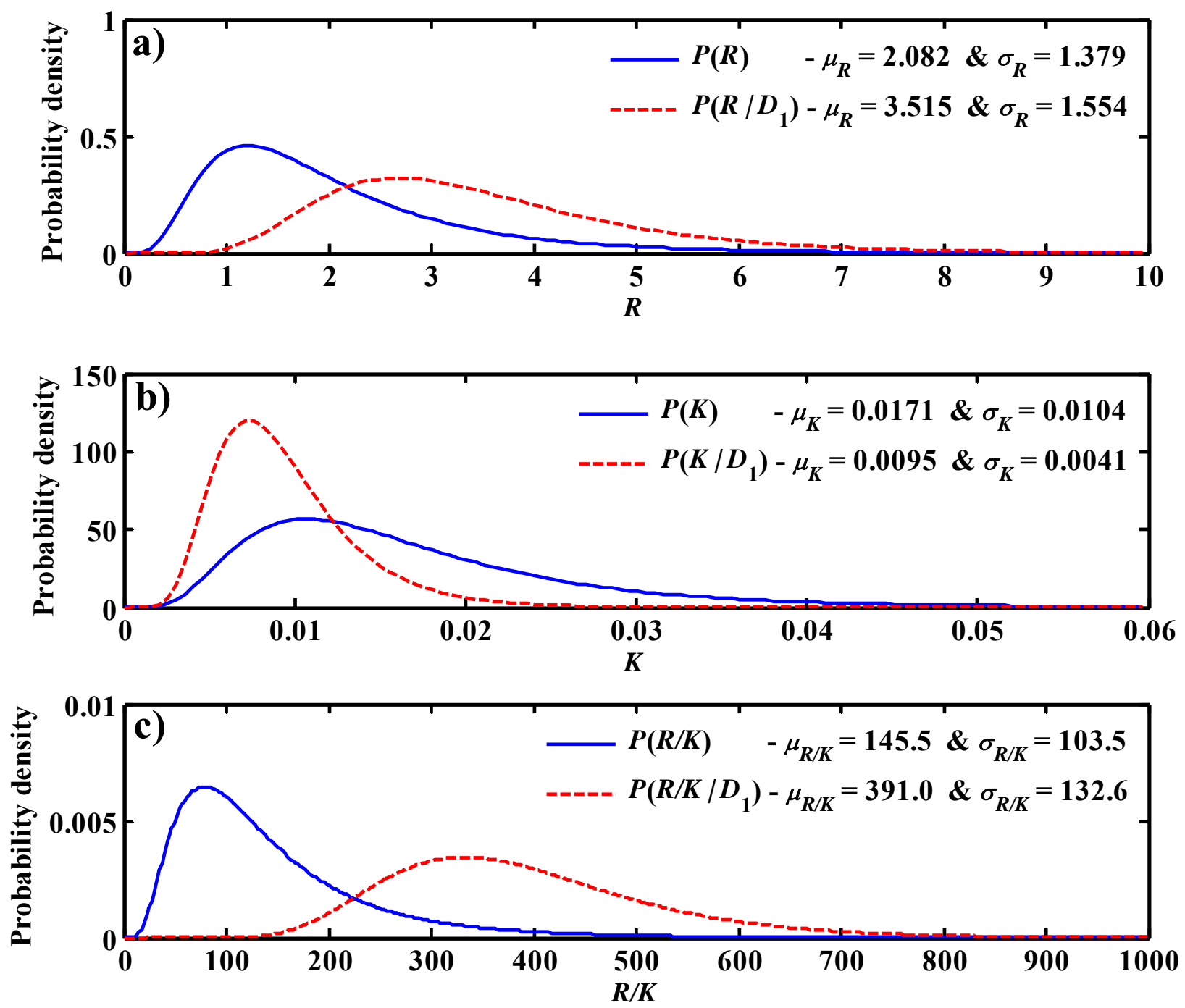

Figure A.5. Results of the MCMC conditioning approach for Milrow, where $D_{1}$ indicates the MT-converted concentrations using the fourth option. 

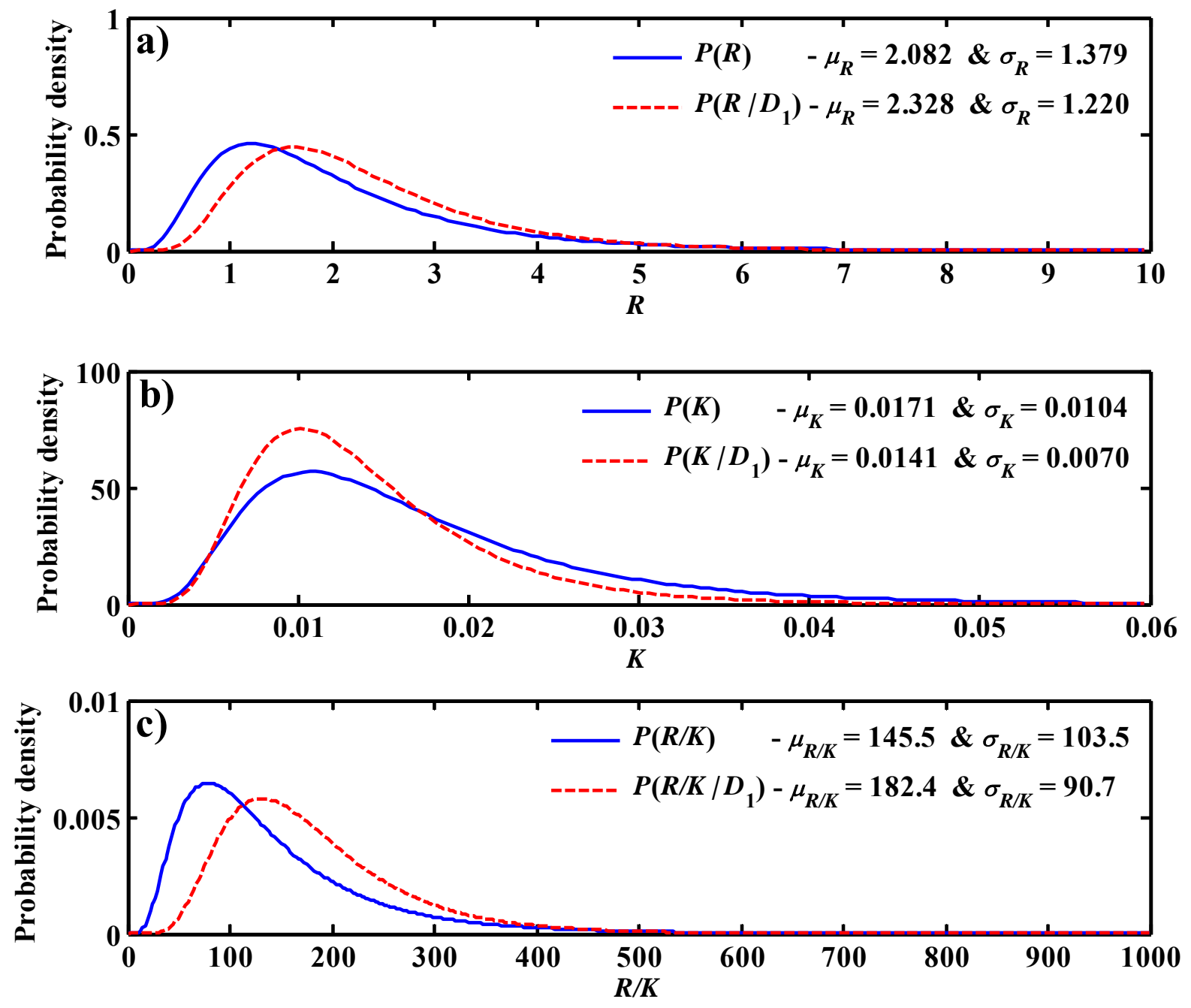

Figure A.6. Results of the MCMC conditioning approach for Milrow, where $D_{1}$ indicates the head data set at UAe-2. 

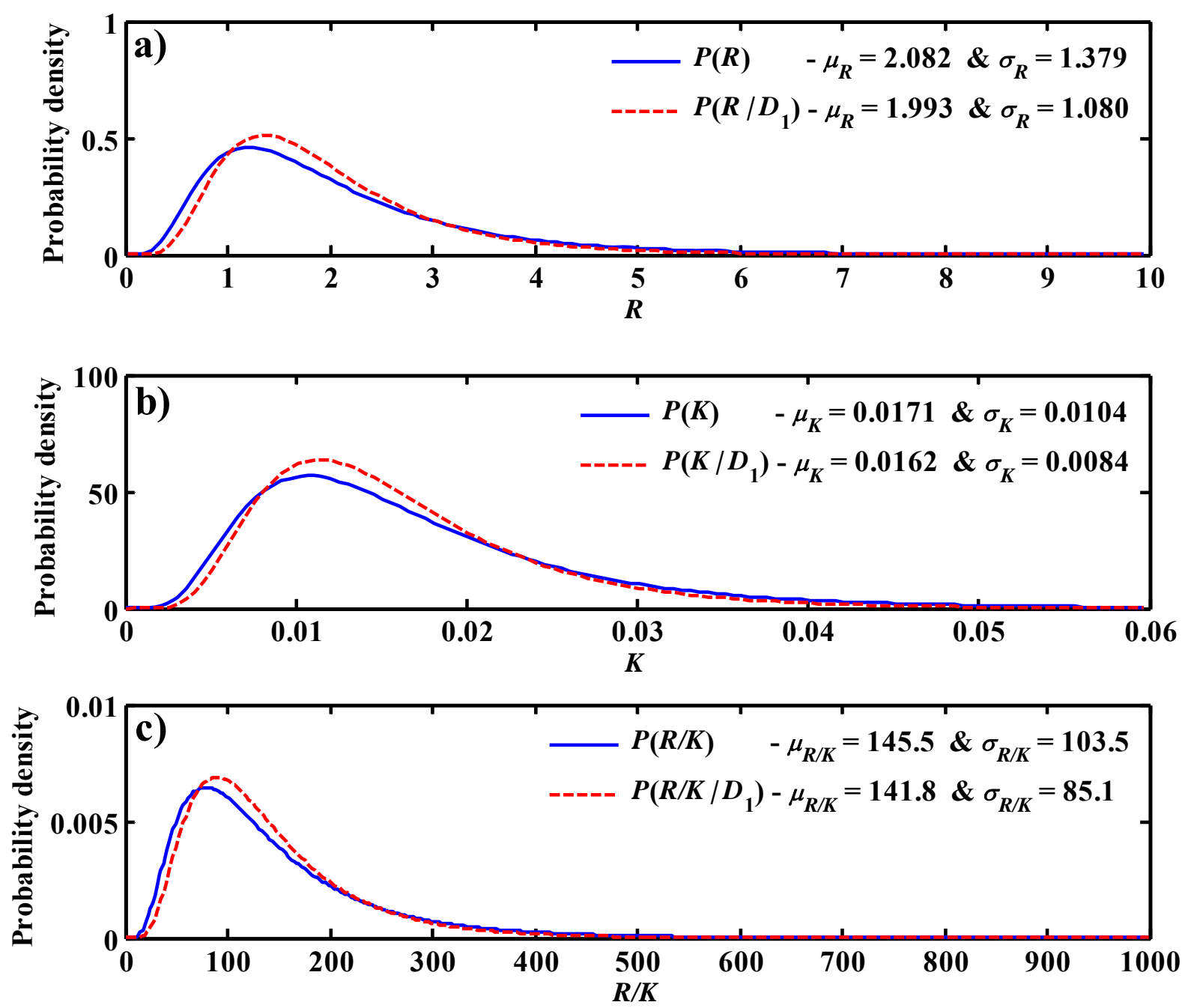

Figure A.7. Results of the MCMC conditioning approach for Milrow, where $D_{1}$ indicates the salinity data set at UAe- 2 . 

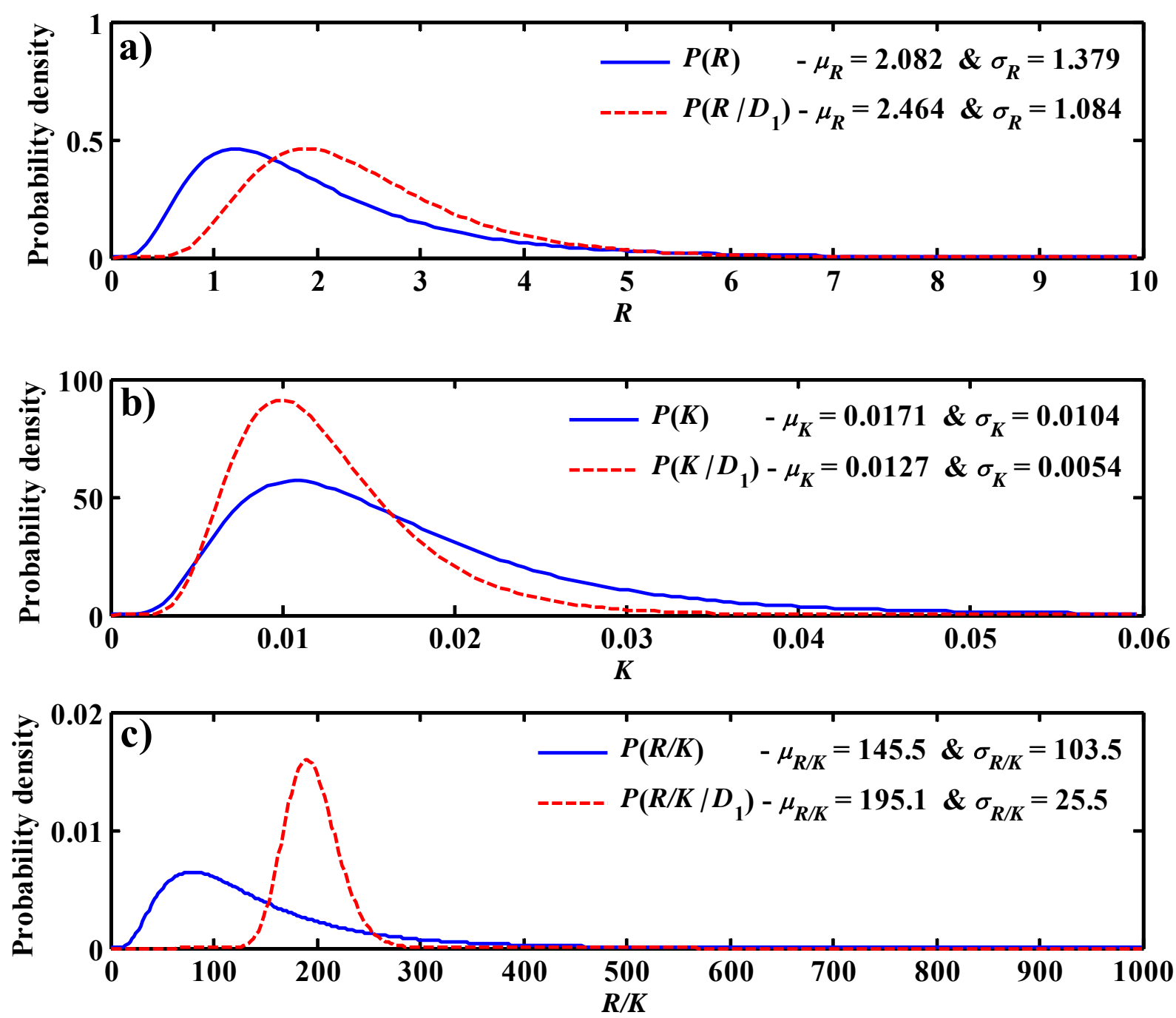

Figure A.8. Results of the MCMC conditioning approach for Milrow, where $D_{1}$ indicates the combined data set where all three data sets (heads at UAe-2, salinity at UAe-2, and MT-converted concentrations using the third option) are used at once. 

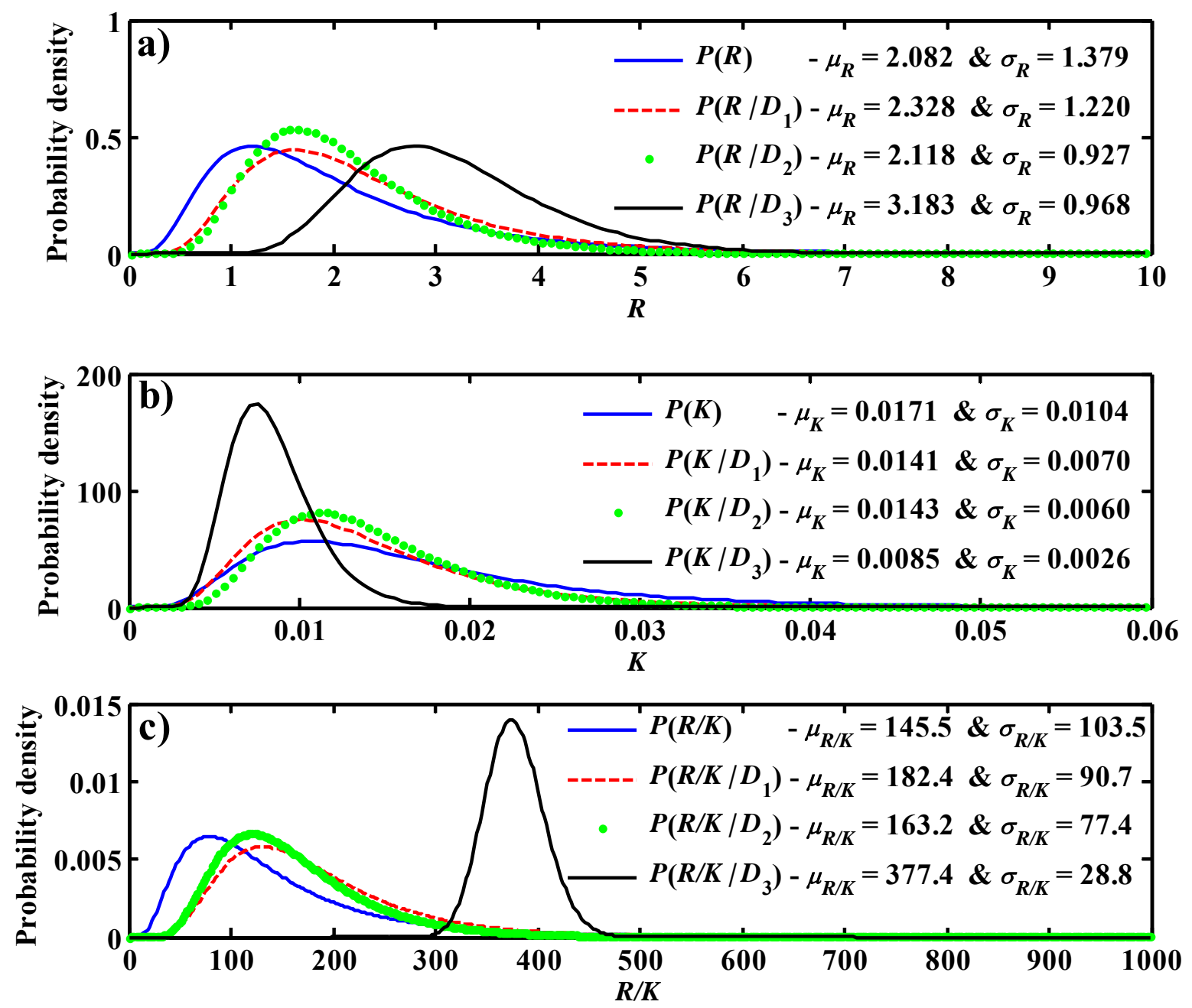

Figure A.9. Results of the sequential MCMC conditioning approach for Milrow (Case 1), where $D_{1}$ indicates the head data set at UAe-2, $D_{2}$ indicates the salinity data at UAe-2, and $D_{3}$ indicates the MT-converted concentrations (third option). 

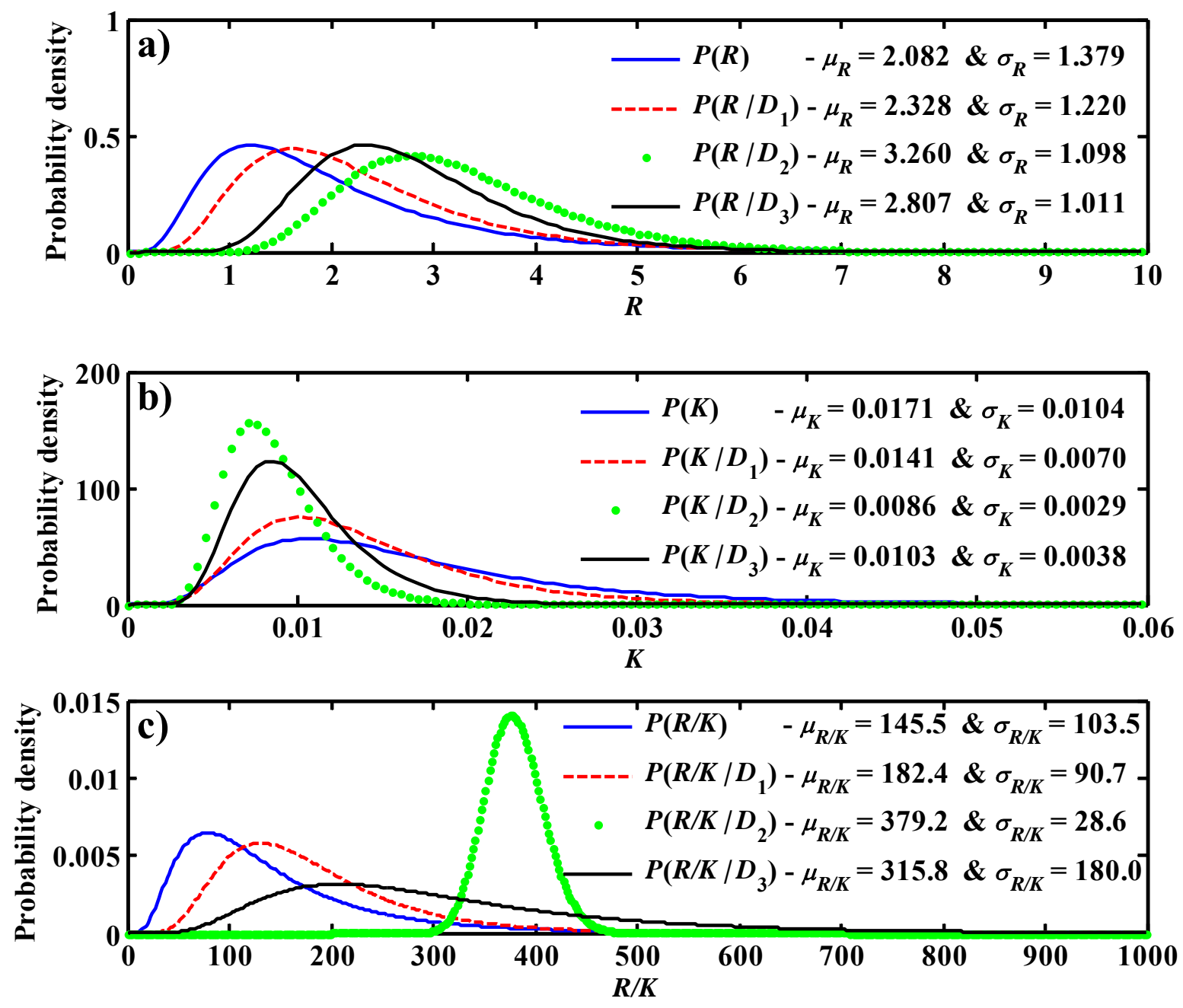

Figure A.10. Results of the sequential MCMC conditioning approach for Milrow (Case 2), where $D_{1}$ indicates the head data set at UAe-2, $D_{2}$ indicates the MT-converted concentrations (third option), and $D_{3}$ indicates the salinity data at UAe-2. 

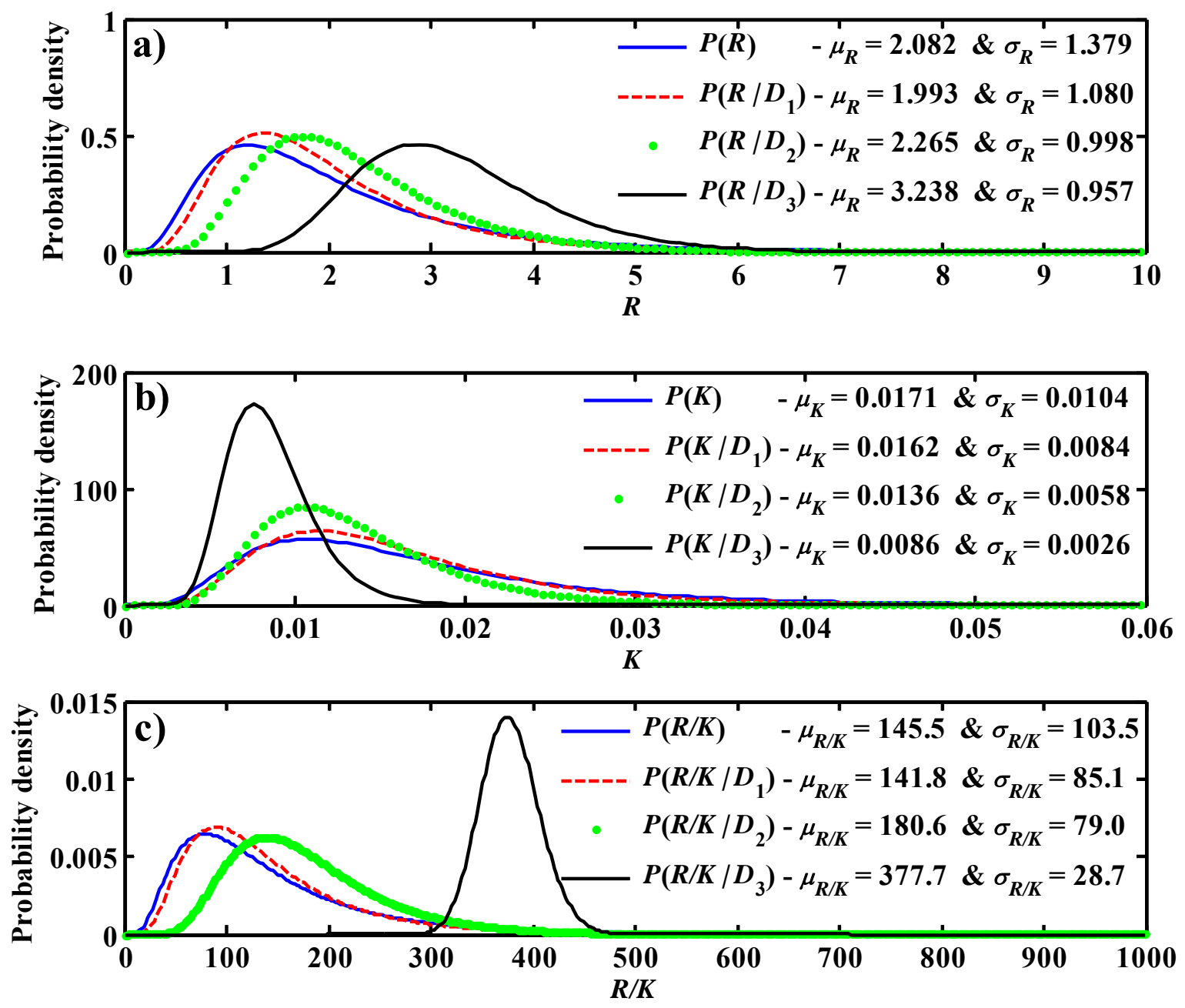

Figure A.11. Results of the sequential MCMC conditioning approach for Milrow (Case 3), where $D_{1}$ indicates the salinity data set at UAe-2, $D_{2}$ indicates the head data at UAe-2, and $D_{3}$ indicates the MT-converted concentrations (third option). 

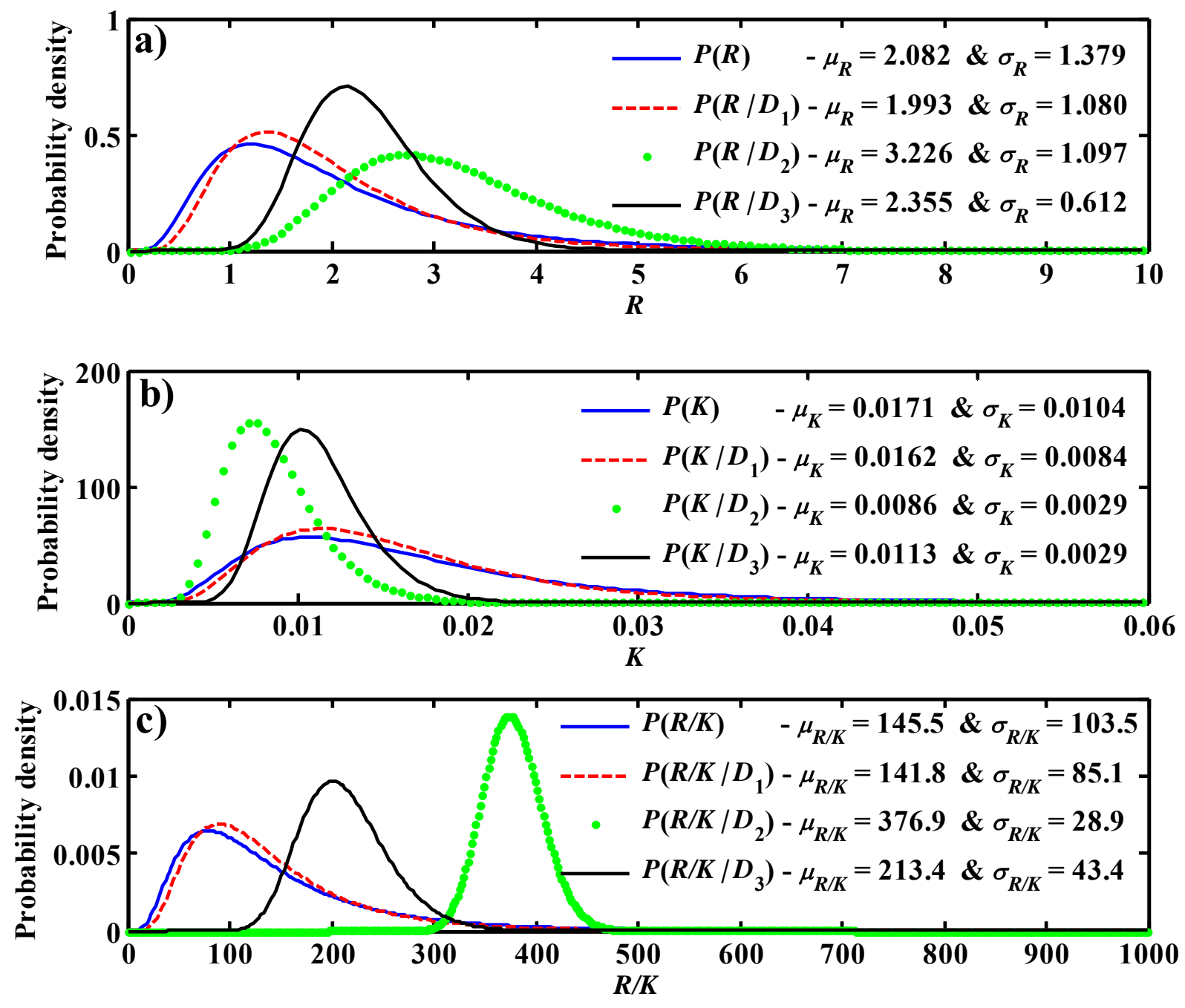

Figure A.12. Results of the sequential MCMC conditioning approach for Milrow (Case 4), where $D_{1}$ indicates the salinity data set at UAe- $2, D_{2}$ indicates the MTconverted concentrations (third option), and $D_{3}$ indicates the head data at UAe-2. 

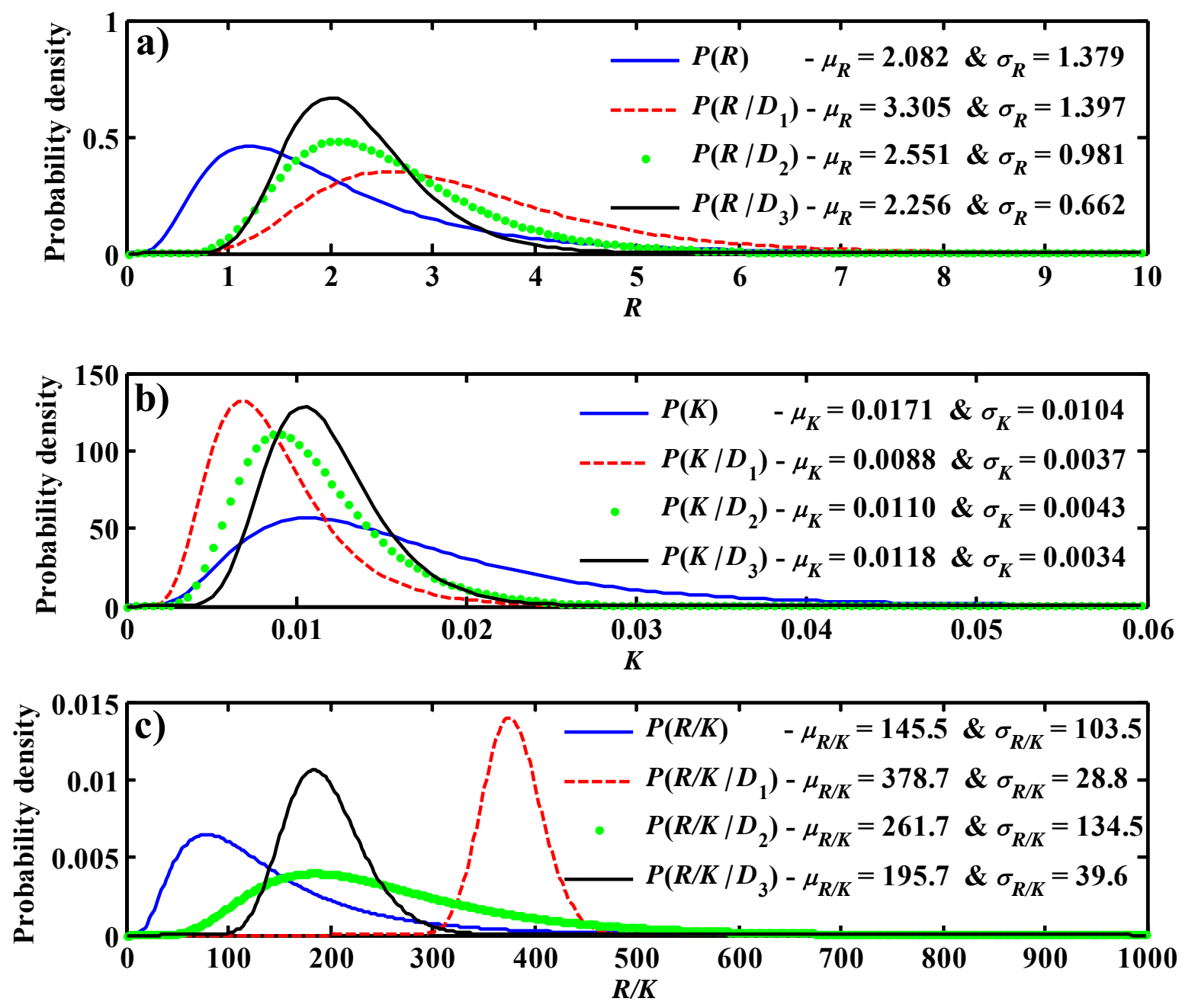

Figure A.13. Results of the sequential MCMC conditioning approach for Milrow (Case 5), where $D_{1}$ indicates the MT-converted concentrations (third option), $D_{2}$ indicates the salinity data set at UAe-2, and $D_{3}$ indicates the head data at UAe-2. 

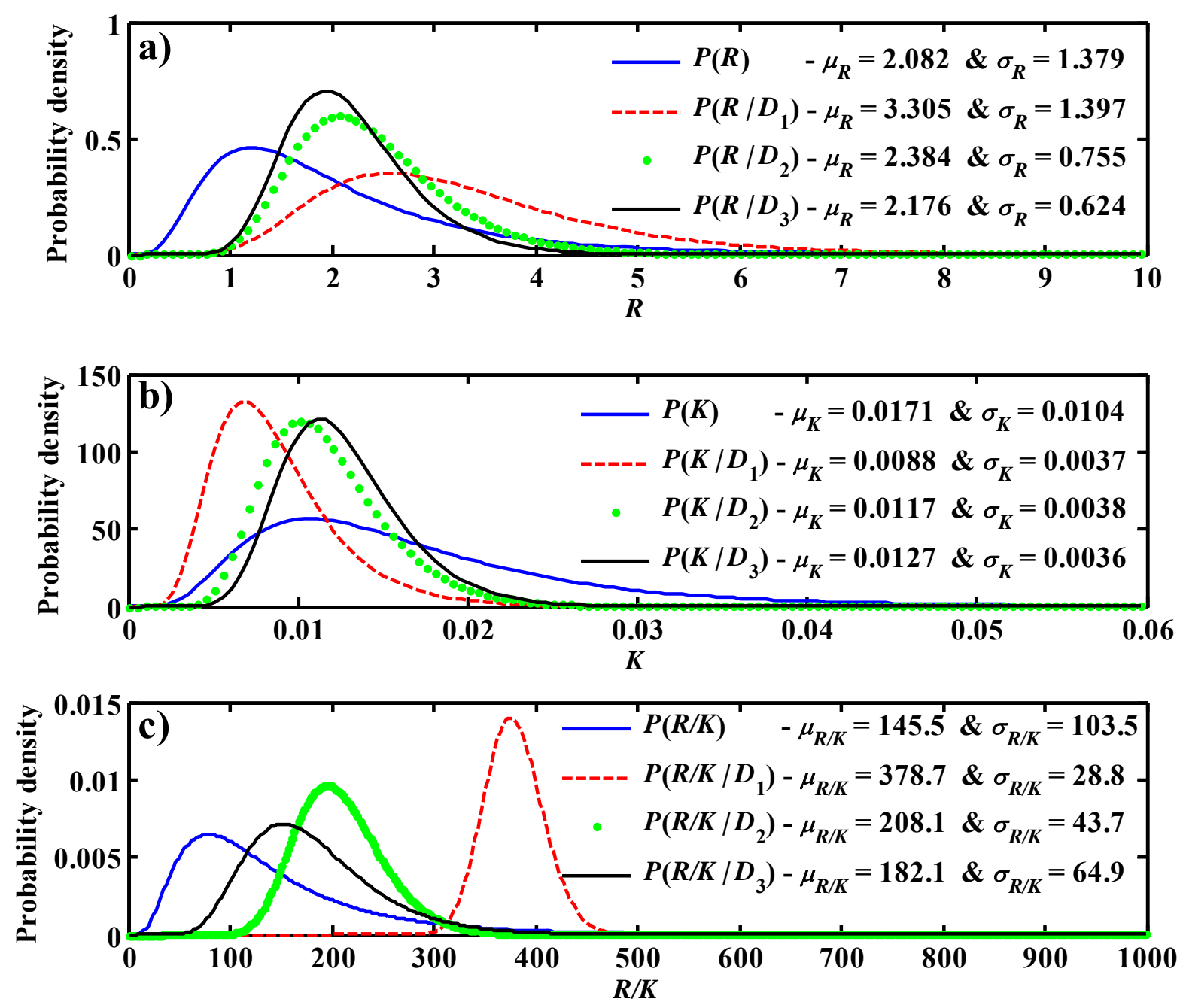

Figure A.14. Results of the sequential MCMC conditioning approach for Milrow (Case 6), where $D_{1}$ indicates the MT-converted concentrations (third option), $D_{2}$ indicates the head data set at UAe-2, and $D_{3}$ indicates the salinity data at UAe-2. 


\section{DISTRIBUTION}

Jenny Chapman

Division of Hydrologic Sciences

Desert Research Institute

755 East Flamingo Road

Las Vegas, NV 89119-7363

Jacqueline Van Lier

Stoller-Navarro Joint Venture

7710 W. Cheyenne

Las Vegas, NV 89128

Janet Appenzeller-Wing, Director

Environmental Restoration Division

Nevada Site Office

National Nuclear Security Administration

U.S. Department of Energy

P.O. Box 98518

Las Vegas, NV 89193-8518

Bruce Hurley

Hydrology Program Manager

Environment, Safety and Health Division

Nevada Site Office

National Nuclear Security Administration

U.S. Department of Energy

P.O. Box 98518

Las Vegas, NV 89193-8518

Tim Echelard

Stoller-Navarro Joint Venture

7710 W. Cheyenne

Las Vegas, NV 89128

Erwin Faqua, Contracting Officer

Acquisition and Financial Assistance

Department

Office of Business Services

NNSA Albuquerque Office

P.O. Box 5400

Albuquerque, NM 87185-5400
Kenneth A. Hoar, Director

Environment, Safety and Health Division

Nevada Site Office

National Nuclear Security Administration

U.S. Department of Energy

P.O. Box 98518

Las Vegas, NV 89193-8518

Richard Hopper

Deputy Laboratory Director

U.S. Environmental Protection Agency

Radiation and Indoor Environments

National Laboratory

4220 S. Maryland Parkway, Bldg. C

Las Vegas, NV 89119

Rick Hutton

Stoller Grand Junction

2597 B 3 / 4 Road

Grand Junction, CO 81503

John Jones

Environmental Restoration Division

Nevada Site Office

National Nuclear Security Administration

U.S. Department of Energy

P.O. Box 98518

Las Vegas, NV 89193-8518

Marjory Jones

Division of Hydrologic Sciences

Desert Research Institute

2215 Raggio Parkway

Reno, NV 89512-1095

Randy Laczniak

U.S. Geological Survey

Water Resources Division

160 N. Stephanie St.

Henderson, NV 89074-8829 
Tom Pauling

Office of Land and Site Management

Office of Legacy Management

U.S. Department of Energy

25973 / 4 Road

Grand Junction, CO 81503

Peter Sanders

Environmental Restoration Division

Nevada Site Office

National Nuclear Security Administration

U.S. Department of Energy

P.O. Box 98518

Las Vegas, NV 89193-8518

David Shafer

Division of Hydrologic Sciences

Desert Research Institute

755 E. Flamingo Road

Las Vegas, NV 89119-7363

Nevada State Library and Archives

State Publications

100 North Stewart Street

Carson City, NV 89710-4285

Archives

Getchell Library

University of Nevada, Reno

DeLaMare Library/262

University of Nevada, Reno

Document Section, Library

University of Nevada, Las Vegas

4505 Maryland Parkway

Las Vegas, NV 89154

Library

Stoller-Navarro Joint Venture

7710 W. Cheyenne, Bldg. 3

Las Vegas, NV 89128
Library

Southern Nevada Science Center

Desert Research Institute

755 E. Flamingo Road

Las Vegas, NV 89119-7363

Technical Library

Nevada Site Office

National Nuclear Security Administration

U.S. Department of Energy

P.O. Box 98518

Las Vegas, NV 89193-8518

Public Reading Facility

c/o Nuclear Testing Archive

Nevada Site Office

National Nuclear Security Administration

U.S. Department of Energy

P.O. Box 98521, M/S 400

Las Vegas, NV 89193-8521

Office of Scientific and Technical Information

U.S. Department of Energy

P.O. Box 62

Oak Ridge, TN 37831-9939

(electronic copy) 